\title{
Contraceptive Use and the Risk of Ovarian Cancer Among Women with a BRCA1 or BRCA2 Mutation
}

by

\author{
Yue Yin Xia
}

A thesis submitted in conformity with the requirements for the degree of Master of Science

Department of Pharmacology and Toxicology University of Toronto

(C) Copyright by Yue Yin Xia 2021 


\title{
Contraceptive Use and the Risk of Ovarian Cancer Among Women with a BRCA1 or BRCA2 Mutation
}

\author{
Yue Yin Xia \\ Master of Science \\ Department of Pharmacology and Toxicology \\ University of Toronto
}

2021

\begin{abstract}
$B R C A$ mutation carriers face a high lifetime risk of developing ovarian cancer. Oral contraceptives are protective in this high-risk population; however, the impact of other types of contraception (e.g. intrauterine devices (IUDs), implants, injections) is unknown. This study evaluated the relationships between type of contraception and risk of developing ovarian cancer among women with a BRCA mutation. Ever use of any contraceptive was significantly associated with reduced risk of ovarian cancer, which was driven by significant inverse associations with oral contraceptives and implants. We observed a similar albeit non-significant effect with use of injections and IUDs. No significant associations were observed between patterns of IUD use and risk of ovarian cancer. These findings support a protective effect of oral contraceptives and implants on risk of ovarian cancer among women with a BRCA1 or BRCA2 mutation. The possible reduced risk with use of injections and IUDs requires further evaluation.
\end{abstract}




\section{Acknowledgements}

First and foremost, a sincere thank you to my supervisor, Dr. Joanne Kotsopoulos for the opportunity to complete my master's degree under her guidance. This research would not have been possible without her expertise and support. Thank you for challenging me to develop new skills, think scientifically and deliver my best work. Furthermore, I would like to thank my committee advisor, Dr. Jennifer Brooks for her enthusiasm and constructive criticism that guided this project. Thank you to Dr. Krista Lanctôt and Dr. Michelle Jacobson for their thoughtful feedback as members of my examination committee.

To the members of the Familial Breast Cancer Research Unit, thank you for training me to perform data collection and ensuring a fun and friendly environment on-site. Namely, Shana Kim, Clotilde Ngwa and Ellen MacDougall, your time, warmth and patience are greatly appreciated. I must also thank Angelina Tryon and Aletta Poll for equipping me with the knowledge and experience to forge a path in genetic counselling. I am grateful for everything I have learned and I am excited to find my place in this field. I sincerely thank my peers, coworkers and lab members, Katie Pullella, Sarah Park, Vasily Giennakeas, Tori Sopik, David Lim, Rod Clark and Christianne Hoey for their knowledge and advice and for making my graduate experience extra special.

Thank you to my dear friends, Annie Xie, Qian Tu and Yan Liu who have seen me through the challenges and triumphs of the past two years with heaping doses of love and silliness. Thank you to Frank Jiao for being my confidante, cheerleader and voice of reason through the thick of it.

To my sister, Anette Xia who is just starting her post-secondary journey - I am so excited to see you blossom from the talented and capable individual you already are. Finally, I would like to thank my parents, Ying Wang and Jinlong Xia for their unconditional love and support. None of this would be possible without you. 


\section{Table of Contents}

Acknowledgements ............................................................................................................... iii

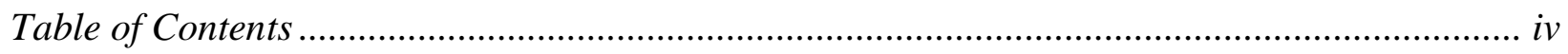

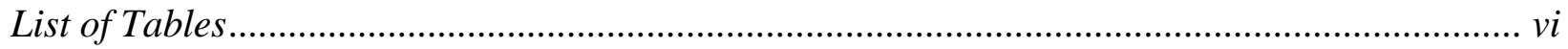

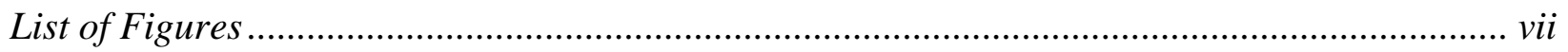

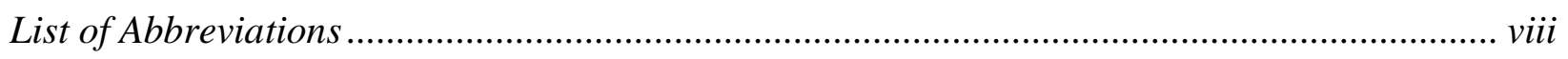

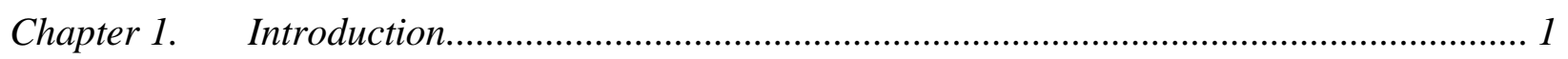

1.1. Breast Cancer Susceptibility Gene-1 (BRCAI) and Breast Cancer Susceptibility Gene-2

$(B R C A 2)$ and Ovarian Cancer ………………………………………………………………...

1.1.1. Overview of $B R C A$-associated Ovarian Cancer.................................................................................. 1

1.1.2. Roles of BRCA Proteins within the Cell.................................................................................... 3

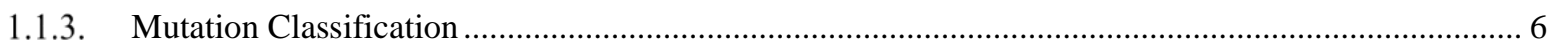

1.1.4. Fallopian Tube as the Site of Origin for Ovarian Cancer ............................................................... 7

1.1.5. Molecular Pathogenesis of $B R C A$-associated Ovarian Cancer ............................................................ 9

1.1.6. Proposed Mechanisms of Ovarian Cancer Pathogenesis ................................................................... 11

1.1.7. Management of Ovarian Cancer Risk in BRCA Mutation Carriers ................................................. 16

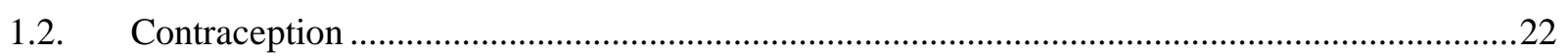

1.2.1. The Hypothalamic-Pituitary-Ovarian Axis and Regulation of the Ovarian Cycle ............................ 22

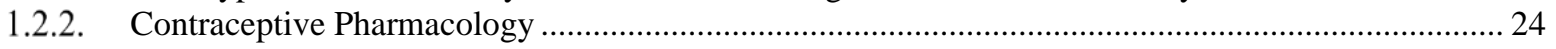

1.2.3. Overview of Contraceptive Methods ....................................................................................... 29

1.3. Contraceptive Use and Ovarian Cancer …………………....................................................

1.3.1. Evidence in the General-Risk Population ..................................................................................... 36

1.3.2. Evidence in High-Risk Populations …………………………………………………………. 46

1.4. The 'Risk Factor' Study ………………………………………………………………...50

Chapter 2. Rationale, Objectives and Hypothesis ………………................................. 51

2.1. Rationale .......................................................................................................

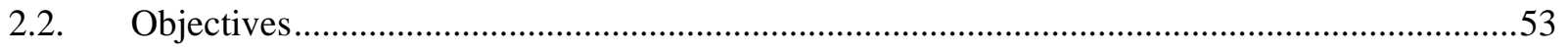

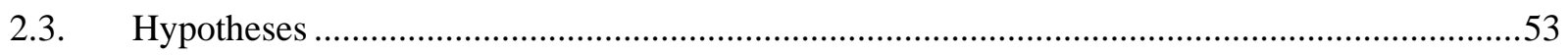

Chapter 3. Methods................................................................................................ 54

3.1. Analysis of Trends in Contraceptive Use in BRCA Mutation Carriers ......................................54

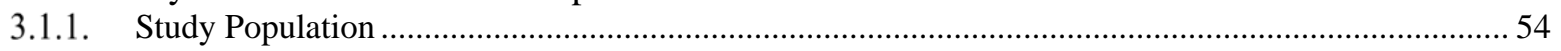

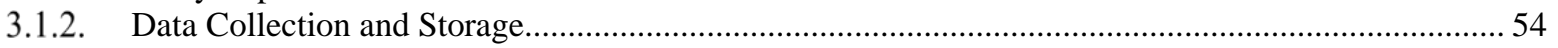

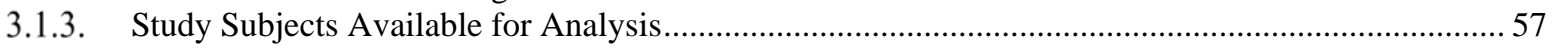

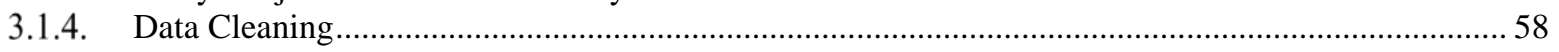

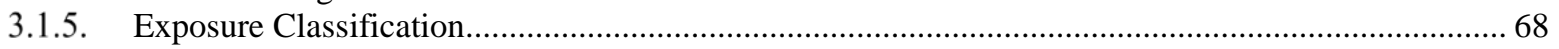

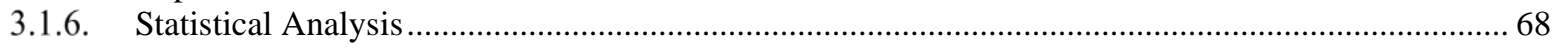

3.2. Analysis of Contraceptive Use and BRCA Ovarian Cancer Risk...............................................69

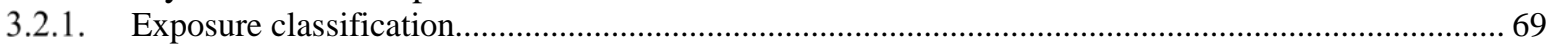

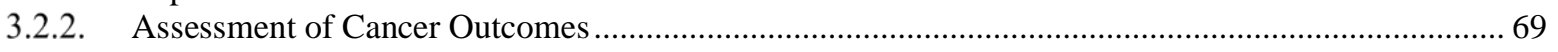

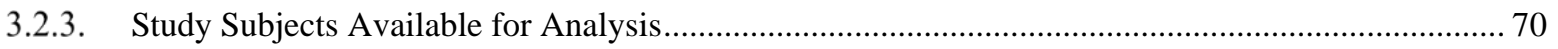

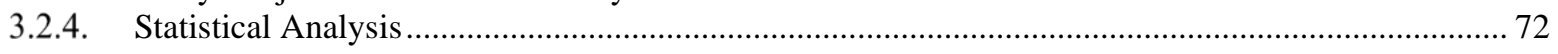

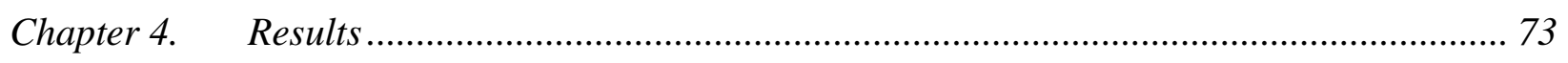


4.1. Trends in Contraceptive use among BRCA Mutation Carriers..............................................73

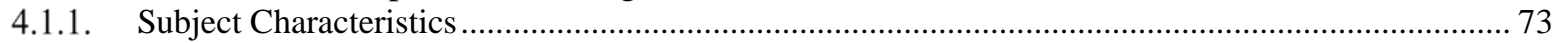

4.1.2. Trends in Use of Various Contraceptive Methods Across Birth Cohorts ........................................... 73

4.2. Associations between Contraceptive Method and BRCA Ovarian Cancer Risk......................79

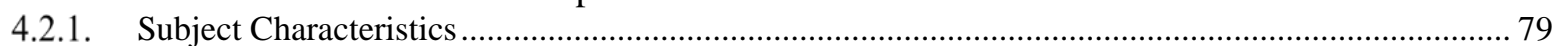

4.2.2. Associations between Methods of Contraceptive Use and Ovarian Cancer Risk............................... 80

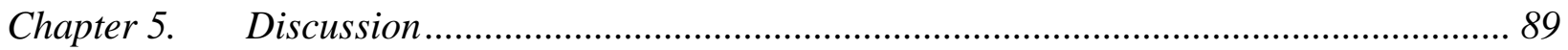

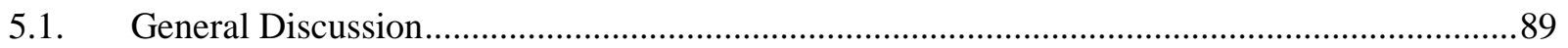

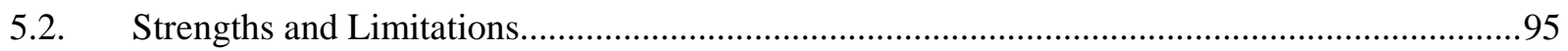

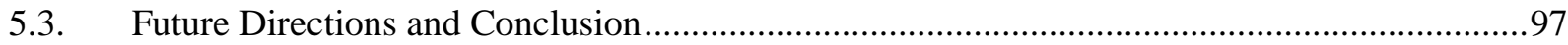

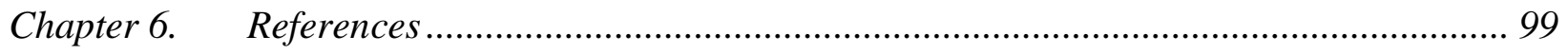

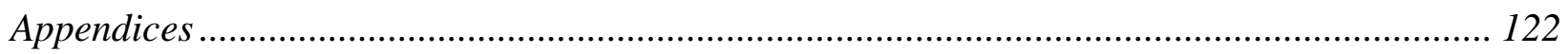

Appendix 1. Research Questionnaire for the Risk Factor Analysis of Breast and Ovarian Cancer .....122

Appendix 2. Follow-up Questionnaire for the Risk Factor Analysis of Breast and Ovarian Cancer ...140

Appendix 3. Informed Consent Form for the Risk Factor Analysis of Breast and Ovarian Cancer.....156

Chapter 7. Copyright Acknowledgements...................................................................... 160 


\section{List of Tables}

Table 1. Summary of known and potential modifiers of sporadic and BRCA-associated ovarian cancer risk 21

Table 2. Classification scheme of progestins used in hormonal contraception ........................ 25

Table 3. Biological activities of various progestogens .................................................... 28

Table 4. Summary of all cohort studies investigating the relationship between oral contraceptive use and ovarian cancer risk in the general population ..................................................... 38

Table 5. Summary of all pooled analyses and meta-analyses investigating the relationship between oral contraceptive use and ovarian cancer risk in the general population ..................... 41

Table 6. Summary of cohort studies, pooled analyses and meta-analyses investigating the relationship between use of an intrauterine device and ovarian cancer risk in the general population

Table 7. Summary of epidemiologic studies investigating the relationship between oral contraceptive use and ovarian cancer risk in BRCA mutation carriers ....

Table 8. REDCap hormonal contraception identification chart

Table 9. Demographic characteristics of contraceptive users with a BRCA1 or BRCA2 mutation

Table 10. Distribution of BRCA1 and BRCA2 mutation carriers who reported ever use of various contraceptive methods by birth cohort. 76

Table 11. Distribution of BRCA1 mutation carriers who reported ever use of various contraceptive methods by birth cohort.

Table 12. Distribution of BRCA2 mutation carriers who reported ever use of various contraceptive methods by birth cohort. 78

Table 13. Comparison of ovarian cancer cases and controls with a BRCA1 or BRCA2 mutation 82 Table 14. Characteristics of users of various contraceptive methods with a BRCA1 or BRCA2 mutation

Table 15. Associations between use of various contraceptive methods and ovarian cancer risk among women with $B R C A 1$ or BRCA2 mutation, stratified by menopausal status.

Table 16. Temporal associations between IUD use and ovarian cancer risk among BRCAl or BRCA2 mutation carriers 87

Table 17. Associations between use of various LARC methods and ovarian cancer risk among women with $B R C A 1$ or $B R C A 2$ mutation, stratified by history of oral contraceptive use .88 


\section{List of Figures}

Figure 1. Functional domains of the human BRCA1 and BRCA2 proteins ............................. 4

Figure 2. Hormonal regulation of the menstrual cycle ................................................... 23

Figure 3. Forest plot summarizing the findings of select case-control and cohort studies

investigating oral contraceptive use and ovarian cancer risk in the general population .............. 41

Figure 4. Contraceptive use questions included in the baseline research questionnaire for the Risk

Factor Analysis of Breast and Ovarian Cancer (Version: 22 May 2018) ................................ 56

Figure 5. Recruitment statistics for a prospective cohort study of contraceptive use and ovarian

cancer risk among BRCA mutation carriers ...................................................................... 57

Figure 6. Recruitment statistics for a matched case-control study of contraceptive use and

ovarian cancer risk among $B R C A$ mutation carriers ........................................................ 71

Figure 7. Trends in proportions of $B R C A 1$ and $B R C A 2$ mutation carriers who reported ever use of various contraceptive methods by birth cohort.......................................................... 76

Figure 8. Trends in proportions of $B R C A 1$ mutation carriers who reported ever use of various

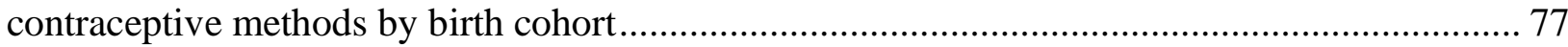

Figure 9. Trends in proportions of BRCA2 mutation carriers who reported ever use of various

contraceptive methods by birth cohort.............................................................................. 78 


\section{List of Abbreviations}

\begin{tabular}{ll} 
BARD1 & BRCA1 associated RING domain protein 1 \\
BMI & Body mass index \\
BRCA1 & Breast Cancer Susceptibility Gene-1 \\
BRCA2 & Breast Cancer Susceptibility Gene-2 \\
BRCT & BRCA1 carboxy-terminal repeats \\
BRIP1 & BRCA1-interacting protein C-terminal helicase 1 \\
CA125 & Cancer antigen 125 \\
CDK & Cyclin-dependent kinase \\
CI & Confidence interval \\
CtIP & CtBP interactive protein \\
DMPA & Depot medroxyprogesterone acetate \\
DSB & Double-stranded break \\
EE & Ethinyl estradiol \\
FSH & Follicle stimulating hormone \\
GnRH & Gonadotrophin-releasing hormone \\
HGSC & High-grade serous ovarian carcinoma \\
HR & Homologous recombination \\
HR & Hazard ratio \\
HRT & Hormonal replacement therapy \\
IARC & International Agency for Research on Cancer \\
IUD & Intrauterine device \\
LARC & Long-acting reversible contraception \\
LH & Luteinizing hormone \\
LNG & Levonorgestrel \\
LOH & Loss of heterozygosity \\
NECC & New England Case Control Study \\
NHEJ & Non-homologous end joining \\
NHS & Nurses' Health Study \\
OC & Oral contraceptive \\
\hline
\end{tabular}


OCCR Ovarian cancer cluster regions

OR Odds ratio

PALB2 Partner and localizer of BRCA2 protein

PRA Progesterone receptor A

PRB Progesterone receptor B

RANK Receptor activator of nuclear factor $\kappa B$

RING Really Interesting New Gene

RR Relative risk

RRBSO Risk-reducing bilateral salpingo-oophorectomy

STIC Serous tubal intraepithelial carcinoma 


\section{Chapter 1. Introduction}

\subsection{Breast Cancer Susceptibility Gene-1 (BRCA1) and Breast Cancer Susceptibility Gene-2 (BRCA2) and Ovarian Cancer}

\subsubsection{Overview of BRCA-associated Ovarian Cancer}

In 2020, ovarian cancer ranked as the eighth most common cancer diagnosis in women worldwide but the leading cause of gynecological cancer death ${ }^{1}$. According to the International Agency for Research on Cancer (IARC), an estimated 313,959 new cases of ovarian cancer are diagnosed annually and 207,252 women die of it ${ }^{1}$. Despite advancements in the understanding and management of ovarian cancer, the majority of patients present with late-stage disease (stage III/IV) and have a five-year survival rate of only $30 \%{ }^{2}$. Data from the World Health Organization have shown minimal improvements in ovarian cancer mortality in recent decades ${ }^{3}$. The greatest declines were observed among developed countries. In the United States, rates dropped approximately $16 \%$ from 5.8 deaths per 100,000 women in 2002 to 4.9 deaths per 100,000 women in 2012. These trends paralleled declines in ovarian cancer incidence rather than improvements in early detection or treatment ${ }^{4}$. To a large extent, the reduced incidence of ovarian cancer has been attributed to changes in reproductive risk factors such as oral contraceptive use, parity, breastfeeding and tubal ligation ${ }^{4}$.

Although the majority of ovarian cancers are sporadic, approximately $25 \%$ of affected women have a hereditary predisposition ${ }^{5}$. Pathogenic variants in the breast cancer susceptibility genes 1 and 2 (BRCA1 and BRCA2) are the strongest known genetic risk factors and account for almost $45 \%$ of cases with a family history of the disease and $15 \%$ of all ovarian cancers ${ }^{6-8}$.

$B R C A 1$ and $B R C A 2$ are tumour-suppressor genes located on chromosome 17q21 and 13q12, respectively. After the initial identification of BRCA1 in 1994 and BRCA2 in 1995, their nuclear protein products have been implicated in several cellular processes required for the maintenance of genome integrity, including DNA repair, cell cycle control, transcriptional regulation, protein ubiquitylation and chromatin remodelling ${ }^{9}$. The role of BRCA1 and BRCA2 in homologous recombination (HR), a high-fidelity pathway of DNA double-stranded break (DSB) repair, 
accounts for their major tumour suppressor activity. In the context of a deleterious germline mutation in $B R C A 1$ or $B R C A 2$, a somatic mutation in the corresponding normal allele can lead to loss of BRCA function, interruption of DNA repair function and initiation of tumourigenesis, according to the two-hit paradigm of tumour development.

Globally, an estimated 1 in 400 to 1 in 800 people are heterozygous carriers of a BRCAl or $B R C A 2$ mutation $^{10}$. Although relatively uncommon, inherited pathogenic mutations in these genes are highly penetrant and predispose individuals to high lifetime risks of breast and ovarian cancer. Compared to an average cumulative risk of $12 \%$ in the general population, the risk of breast cancer by age 80 has been estimated at $72 \%$ in BRCA1 mutation carriers and $69 \%$ in $B R C A 2$ mutation carriers ${ }^{11}$. Women who inherit a deleterious $B R C A$ mutation also face an increased lifetime risk of developing ovarian cancer, compared to a $1.2 \%$ risk in the general population. This is estimated at $44 \%$ for $B R C A 1$ and $17 \%$ for $B R C A 2$, corresponding to average annual risk estimates of $0.86 \%$ and $0.37 \%$, respectively ${ }^{11,12}$. Lesser elevated risks have also been reported for cancers of other sites such as the prostate, pancreas and colon ${ }^{13}$.

Germline BRCA mutations are associated with ovarian cancers of distinct clinicopathological features. On average, mutation carriers are diagnosed at an earlier age compared to sporadic cases of ovarian cancer. The median age at diagnosis of ovarian cancer in the general population is 63 years, whereas the median age at diagnosis for $B R C A 1$ and $B R C A 2$ mutation carriers is estimated at 54 and 59.5 years, respectively ${ }^{11}$. The majority of $B R C A$-associated ovarian cancers are of high-grade serous histology (67\%) although endometrioid (12\%) and clear cell carcinomas $(2 \%)$ have also been reported ${ }^{14}$. Most carriers present at advanced stage, with over $80 \%$ of cases diagnosed at stage III/IV, by which point the disease has spread to peritoneal surfaces ${ }^{15,16}$. Shortterm survival outcomes are more favourable for BRCA-positive women compared to mutationnegative women due to greater tumour sensitivity to platinum therapy ${ }^{17,18}$; however, this response does not predict long-term survival. In a study of 1,421 ovarian cancer cases, 10-year survival estimates were $43 \%$ for noncarriers, $57 \%$ for $B R C A 1$ carriers and $69 \%$ for $B R C A 2$ carriers, suggesting significantly worse long-term survival among carriers ${ }^{19}$. 


\subsubsection{Roles of BRCA Proteins within the Cell}

BRCAl encodes a large multi-domain protein spanning 1,864 amino acids in length ${ }^{20}$ (Figure 1). The BRCA1 protein interacts with multiple protein binding partners through three major domains: the Really Interesting New Gene (RING) finger domain at the N-terminus, a central coiled-coil domain, and tandem BRCA1 carboxy-terminal repeats (BRCTs) at the C-terminus. BRCA1 interacts with its obligatory partner, BRCA1-associated RING domain protein 1 (BARD1) through common RING domains. The BRCA1-BARD1 heterodimer has E3 ubiquitin ligase activity, which is involved in DNA repair and tumour suppression ${ }^{121}$. The BRCT repeats mediate interactions between BRCA1 and several DNA damage response proteins including abraxas CtBP interactive protein (CtIP) and BRCA1-interacting protein C-terminal helicase 1 (BRIP1) through binding of phosphorylated serine at the pSXXF motif ${ }^{22}$. The coiled-coil domain of BRCA1 binds a homologous domain in the partner and localizer of BRCA2 protein (PALB2), which facilitates recruitment of BRCA2 during HR. These structural domains flank an intrinsically disordered central region encoded by exon 11 that contains several DNA-damageinduced phosphorylation sites to facilitate the formation of various BRCA1 protein complexes ${ }^{23}$.

$B R C A 2$ does not share a high degree of sequence homology with $B R C A 1$, but both proteins are functionally related through involvement in the HR pathway (Figure 1) 22 . BRCA2 encodes a 3,418 amino acid product that associates with the HR-related proteins, PALB2 and RAD51. At the N-terminus, BRCA2 executes PALB2 binding between amino acids 21-30. Centrally, eight $\mathrm{BRC}$ repeats mediate binding and recruitment of the recombinase, RAD51, which is essential for the initiation of DNA repair. Downstream, BRCA2 contains a DNA-binding domain as well as a cyclin-dependent kinase (CDK) phosphorylation site at S3291 of the C-terminus, which is also important for RAD51 binding 22 . 

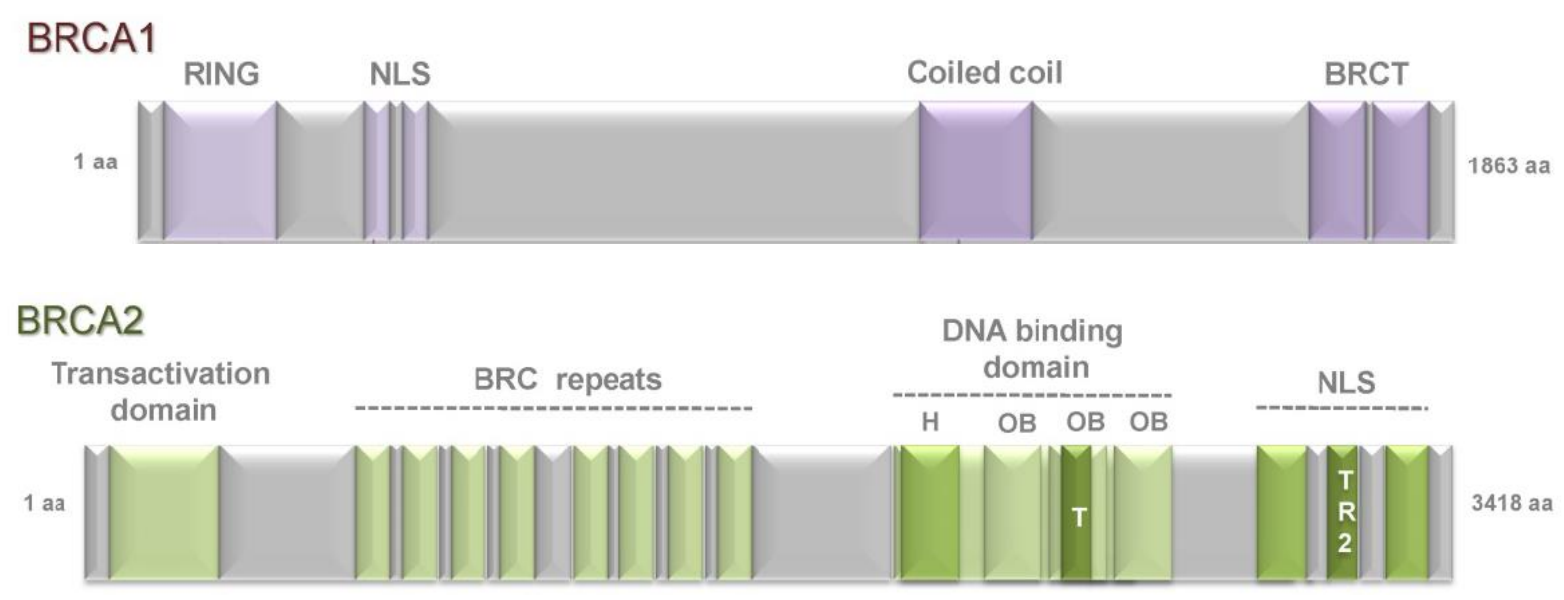

Figure 1. Functional domains of the human BRCA1 and BRCA2 proteins

Footnote to Figure 1: BRCAl is an 1,863-amino acid protein composed of 23 exons. Functional domains depicted as violet boxes in BRCAl include the Really Interesting New Gene (RING) finger domain, two nuclear localization signals (NLS), a coiled-coil domain and two BRCA1 C-terminal (BRCT) domains. BRCA2 is a 3,418-amino acid protein comprised of 27 exons. Functional domains depicted as green boxes in BRCA2 include the transcriptional activation domain, eight BRC motifs, a conserved helical $(\mathrm{H})$ domain, three oligonucleotide-binding $(\mathrm{OB})$ folds, a tower $(\mathrm{T})$ domain and two NLS'. Domain names are denoted above.

Reproduced from Gorodetska I, Kozeretska I, Dubrovksi A. BRCA Genes: The Role in Genome Stability, Cancer Stemness and Therapy Resistance. J Cancer. 2019; 10(9): 2109-2127 by permission from Ivyspring International Publisher.

\subsubsection{Role of BRCA1 and BRCA2 in Homologous Recombination}

$B R C A 1$ and $B R C A 2$ are considered 'caretaker' genes that serve to maintain genome integrity and tumour suppression in the cell. Through its various functional domains, BRCA1 has pleiotropic roles in multiple DNA repair pathways, including HR, non-homologous end joining (NHEJ) and single-strand annealing, as well as cell cycle control, chromatin remodelling and transcriptional regulation $^{22}$. Conversely, BRCA2 is primarily involved in HR in complex with RAD51. The roles of BRCA1 and BRCA2 in the repair of DNA DSBs by HR are well-described and thought to underly their tumour suppressor activity. DSBs can arise from exogenous exposure to ionizing radiation or chemotherapeutic agents as well as endogenous sources such as reactive oxygen species or replication error ${ }^{22}$. Repair of DSBs can occur by HR or NHEJ. Whereas NHEJ is error-prone and may result in insertions or deletions, HR is a high-fidelity mechanism that uses the homologous sister chromatid as a template for repair ${ }^{20}$. BRCA1 forms three main complexes that participate in the early stages of the HR pathway. BRCA2 participates downstream by mediating assembly of RAD51 on single-stranded DNA. The BRCA1-A complex consists of 
BRCA1, Rap80, Abraxas, NBA1, BRE and BRCC36 and targets BRCA1 to sites of DNA damage by associating with ubiquitinated histones at the DSB ${ }^{23}$. BRCA1 also associates with the resection complex MRN and recruits the resection factor, CtIP to form the BRCA1-C complex. BRCA1-C catalyzes DNA end resection to yield a 3' single-stranded DNA tail, signalling the earliest commitment step of HR. BRCA1 associates with BRCA2 through the bridging protein, PALB2. The BRCA1-PALB2-BRCA2 holoenzyme complex, otherwise known as BRCC, coordinates RAD51-loading of single-stranded overhangs, which guides strand invasion of the intact homologous chromosome and formation of a homologous duplex ${ }^{24}$. DNA synthesis proceeds from the 3' end and restores the strand's original sequence. The displacement loop is subsequently resolved to generate crossover or non-crossover products. 


\subsubsection{Mutation Classification}

To date, over 1,600 unique mutations have been identified in $B R C A 1$ and over 1,800 unique mutations have been reported in $B R C A 2^{10}$. Approximately $80 \%$ of known pathogenic mutations in these genes generate a premature stop codon resulting in a truncated and inactive form of the protein ${ }^{25-27}$. These include nonsense mutations, frameshift mutations, splicing site mutations and large deletions ${ }^{28}$. While most truncating mutations are dispersed across the lengths of both genes, deleterious missense mutations have been observed to cluster in specific regions of BRCAl and $B R C A 2$. The most frequently reported mutations in BRCAl occur at the BRCT and RING finger domains, whereas mutations in BRCA2 tend to surround the BRC repeats and $\mathrm{OB}$ folds ${ }^{29}$.

Mutation-specific cancer risks have also been linked to key regions of BRCAl and BRCA2. Several ovarian cancer cluster regions (OCCR) have been identified in or near exon 11 of both genes, bounded by c.1380 to c.4062 in BRCAl as well as c.3249 to c.5681 and c.6645 to c.7471 in $B R C A 2^{30}$. Mutations within the OCCR are associated with a significant relative increase in ovarian cancer risk to breast cancer risk ${ }^{30}$.

Several founder mutations in $B R C A 1$ and $B R C A 2$ have reported at high frequencies in specific populations. The most striking examples are observed among families of European (Ashkenazi) Jewish ancestry, in which three founder mutations (185delAG and 5382insC in BRCAl; 6174 delT in BRCA2) account for nearly $80 \%$ of all $B R C A$ mutations identified in this population ${ }^{31}$. Other founder mutations have been established for European (Polish, Icelandic, Norwegian, Swedish, Italian) and non-European (French Canadian, Hispanic, Chinese, Japanese, Malaysian) populations ${ }^{32}$. 


\subsubsection{Fallopian Tube as the Site of Origin for Ovarian Cancer}

Ovarian cancer was traditionally thought to arise de novo from the ovarian surface epithelium or from cortical inclusion cysts within the ovarian parenchyma ${ }^{33}$. However, several limitations have emerged to challenge this theory of cellular origin. Despite decades of effort, no ovarian precursor lesions had been identified at these sites $^{34}$. Moreover, the majority of ovarian tumours are composed of Müllerian-type epithelium that is not present in the ovary ${ }^{35}$. The major histologic subtypes of ovarian cancer (serous, endometrioid and mucinous) do not resemble cell types of the ovary. Rather, they are morphologically similar to epithelia of the fallopian tube, endometrium and cervix, respectively ${ }^{35}$.

In 1999, Dubeau was the first to argue that what seemed to be primary ovarian epithelial tumours may actually originate from derivatives of the Müllerian tract including the fallopian tube and endometrium ${ }^{35}$. Recent morphological and molecular findings have added support to a dualistic model of ovarian carcinogenesis in which ovarian cancers predominantly arise from the endometrial or fallopian tube epithelium and spread to the ovary secondarily ${ }^{36}$. According to this model, the various histologic subtypes of ovarian cancer may be classified as Type I or Type II with distinct clinicopathological profiles, molecular features and tissues of origin. Type I carcinomas, such as clear cell, endometrioid, mucinous and low-grade serous carcinomas, arise from borderline serous tumours or endometriosis ${ }^{37}$. Type II carcinomas mainly comprise highgrade serous ovarian carcinoma (HGSC), the most common type of epithelial ovarian cancer, and develop from the secretory epithelium of the distal fallopian tube as well as the ovary ${ }^{37}$.

Support for the tubal origin of HGSC is based on a wealth of clinical, epidemiological, histological and molecular evidence that has emerged in the last two decades. Beginning in the early 2000s, studies of asymptomatic BRCA mutation carriers undergoing prophylactic salpingooophorectomy have identified a high frequency of early serous carcinomas localized to the distal fallopian tube. Between $57 \%$ and $100 \%$ of occult malignancies exhibited fallopian tube involvement at the fimbriated end ${ }^{38-42}$. Similar lesions were later discovered in the fallopian tubes of $50 \%$ to $60 \%$ of women with sporadic HSGC but not among other histologic subtypes ${ }^{43,44}$. Termed serous tubal intraepithelial carcinomas (STICs), these non-invasive 
carcinomas of the fallopian tube represent the immediate precursor of HGSC for both $B R C A$ mutation-positive and $B R C A$ mutation-negative women. These findings were validated by genetic and molecular studies that further confirmed the link between STICs and HGSC. The detection of identical TP53 mutations between STICs and concomitant ovarian HGSCs support a clonal relationship and direct evolutionary descent from STICs ${ }^{45,46}$. Molecular profiling studies have demonstrated that the gene expression signature of HGSC resembles that of the fallopian tube epithelium more than the ovarian surface epithelium ${ }^{47}$. Moreover, a premalignant precursor of STIC has been identified in the fallopian tube that shares a common fimbrial location, cell type, evidence of DNA damage and TP53 mutations with STICs. Comprised of short stretches of benign fallopian tube epithelium that strongly express p53, 'p53 signature' lesions represent the earliest known molecular event in HGSC development.

Based on these observations, the proposed pathogenic sequence initiates with genotoxic stress at the fallopian tube epithelium, leading to DNA damage, induction of TP53 mutations and clonal expansion of a 'p53 signature'. The second step involves further malignant transformation, giving rise to a STIC with proliferative activity. STIC has the potential to invade the tubal stroma or exfoliate onto the ovarian surface and develop HGSC ${ }^{48}$. 


\subsubsection{Molecular Pathogenesis of $B R C A$-associated Ovarian Cancer}

\subsubsection{Two-hit Hypothesis}

The molecular pathogenesis of hereditary breast and ovarian cancer is traditionally believed to follow the 'two-hit' model proposed by Knudson. BRCA1 and BRCA2 represent canonical tumour suppressor genes characterized by an autosomal dominant pattern of inheritance ${ }^{49}$. BRCA mutation carriers typically inherit a single mutant copy in the germline and exhibit somatic loss of the remaining normal (wild-type) BRCA allele in familial breast and ovarian tumours ${ }^{50-52}$. Loss of heterozygosity ( $\mathrm{LOH})$ is considered the rate-limiting step in $B R C A$-related tumour development and occurs through genetic or epigenetic inactivation of BRCA activity ${ }^{53}$. An inherited $B R C A$ defect may accelerate $B R C A$ LOH and contribute to the earlier onset of disease seen in carriers.

\subsubsection{BRCA Haploinsufficiency}

Accumulating evidence also supports a mechanism of $B R C A$ haploinsufficiency as a driver of hereditary breast and ovarian tumour predisposition. Retention of the normal allele has been observed in a subset of germline BRCA1 and BRCA2 ovarian tumours ${ }^{54}$. Work in breast cancer cell lines reveal phenotypic effects of $B R C A$ heterozygosity that include spontaneous recombination events and impaired $\mathrm{HR}$, which increase the risk of additional cancer-promoting mutations $^{55-57}$. In animal studies, Brcal heterozygous mice had a higher incidence of ovarian tumour formation upon exposure to ionizing radiation, indicating a specific effect on ovarian cells $^{58}$. Thus, BRCA haploinsufficiency may cause genomic instability and promote loss of the wild-type allele.

\subsubsection{TP53 Loss in Early BRCA-associated Tumourigenesis}

Biallelic inactivation of Brcal or Brca2 is embryonically lethal in mouse models, and BRCA deficiency in human cells is similarly thought to result in cell cycle arrest or cell death due to induction of DNA-damage dependent cell cycle checkpoints ${ }^{22,59}$. This raises the question of how $B R C A$-deficient cells may escape apoptosis and accumulate chromosomal abnormalities that promote tumourigenesis. Emerging evidence indicates that somatic inactivation of checkpoint 
control genes such as TP53 may be a pre-requisite for BRCA gene inactivation in early neoplastic development. Consistent with this hypothesis, $92 \%$ of HGSCs of the ovary arising in BRCA mutation carriers harbour mutations in TP53 ${ }^{60}$. Additionally, TP53 mutations were detected in the absence of BRCA LOH in the non-neoplastic fallopian tube epithelium of carriers, whereas all early tubal neoplasias demonstrated loss of the wild-type $B R C A$ allele ${ }^{61}$. Taken together, the data suggest that TP53 loss is an essential early event in BRCA-associated ovarian cancer progression, allowing for the propagation of $B R C A$-deficient cells. 


\subsubsection{Proposed Mechanisms of Ovarian Cancer Pathogenesis}

The $B R C A$ genes are ubiquitously expressed and participate in general cellular mechanisms, thus the predisposition toward site-specific cancers remains unclear. Several hypotheses have been put forward to account for the particular increased risk of ovarian cancer seen among BRCAI and BRCA2 mutation carriers. In these models, the ovary and fallopian tube accumulate specific hormonal and inflammatory exposures through ovulation that may encourage the development of ovarian cancer in a BRCA-deficient backdrop; however, these etiologic pathways overlap as physiologically interrelated events during ovulation.

\subsubsection{Incessant Ovulation Hypothesis}

In 1971, Fathalla postulated that ovulation contributes to the etiology of ovarian cancer through repeated minor trauma to the ovarian surface epithelium ${ }^{62}$. Rupture of the ovarian follicle is followed by rapid proliferation of ovarian epithelial cells during postovulatory wound repair. According to the "incessant ovulation" hypothesis, repeated exposure of the ovarian surface epithelium to follicular rupture and repair through menstrual cycles predisposes ovarian epithelial cells to DNA damage and malignant transformation ${ }^{63}$. The incessant ovulation hypothesis gained further support from studies of comparative oncology demonstrating the rarity of epithelial ovarian carcinomas in animals that limit ovulation to breeding seasons interspersed by non-ovulatory rest periods ${ }^{62}$. In humans, conditions associated with a greater lifetime number of ovulatory episodes, such as early menarche, late menopause and nulliparity, correspond to greater risk of developing ovarian cancer ${ }^{64}$. On the other hand, conditions in which ovulation is suppressed, such as multiple pregnancies, oral contraceptive use and breastfeeding have been reported to lower risk of developing ovarian cancer (described in Section 1.1.7) ${ }^{64}$.

The incessant ovulation hypothesis still holds in light of a tubal origin of ovarian cancer (see Section 1.1.4). Emphasis shifts to the impact of follicular fluid, which is released alongside the cumulus-oocyte complex and bathes the ovarian surface epithelium and distal fallopian tube immediately after ovulation ${ }^{65}$. Mature follicular fluid is abundant in inflammatory cytokines, chemokines, free radicals, growth factors and reactive oxygen species ${ }^{66}$. In vitro studies 
demonstrate that exposure of fimbrial epithelial cells to follicular fluid upregulates inflammatory signalling, genotoxic stress and DNA damage ${ }^{67,68}$.

\subsubsection{Inflammation Hypothesis}

Many features of the ovulatory process resemble that of an inflammatory process ${ }^{69}$. Ovulation is initiated by a midcycle luteinizing hormone $(\mathrm{LH})$ surge, which stimulates granulosa and theca cells to produce cytokines, chemokines, prostaglandins, steroid hormones and other inflammatory mediators. In turn, there is increased migration of immune cells (e.g. neutrophils, monocytes, macrophages, mast cells) to the follicle that potentiates local apoptosis, follicular rupture and oocyte release ${ }^{70}$. Exposure of tubal and ovarian epithelial cells to follicular fluid can also be carcinogenic, as described above. In the luteal phase, local macrophages secret prostaglandins, reactive oxygen species and TNF- $\alpha$ to stimulate apoptosis of corpus luteum cells $^{70}$. Thus, ovulation is considered an inflammatory risk factor that results in cyclic exposure of the fallopian tubes and ovaries to a pro-inflammatory milieu. Local elevation of reactive oxygen species, prostaglandins, cytokines and other inflammatory substances during ovulation enhances the risk of mutagenesis. Lending further support, increased ovarian cancer risk has been observed with other inflammatory factors, such as use of talcum powder and pelvic inflammatory conditions, while use of nonsteroidal anti-inflammatory drugs and tubal ligation are related to reduced risk of ovarian cancer ${ }^{71}$.

More recently, Vercellini proposed the incessant menstruation hypothesis as an extension of inflammation hypothesis that considers the retrograde flow of menstrual blood through the fallopian tubes collecting in the peritoneal cavity ${ }^{72}$. In this model, retrograde menstruation from the endometrial cavity is the source of fallopian tube inflammation. The abundance of free heme is broken down to catalytic iron, which initiates the formation of free radicals and increases genotoxic oxidative stress ${ }^{70}$. This theory is consistent with the reduced risk of ovarian cancer associated with tubal ligation in the general population and in some high-risk groups as well as the increased risk of ovarian cancer linked to endometriosis ${ }^{73-77}$. 


\subsubsection{Gonadotropin Hypothesis}

The carcinogenic role of key reproductive hormones has also been explored through two hormonal hypotheses of ovarian tumourigenesis.

The gonadotropin hypothesis implicates high circulating levels of pituitary gonadotropins with increased risk of developing epithelial ovarian cancer. Gonadotropins may directly stimulate the ovarian surface epithelium by activating LH- and follicle stimulating hormone (FSH)-responsive genes that promote cellular proliferation and malignant transformation ${ }^{78}$. Gonadotropins also induce ovarian steroidogenesis, which can indirectly influence malignant transformation though increased estrogenic stimulation of the ovarian surface epithelium via a paracrine mechanism. Indeed, protective factors against ovarian cancer such as pregnancy and oral contraceptive use are associated with lower circulating gonadotropins ${ }^{79}$. Nonetheless, experimental data on the direct effect of gonadotropin hormones on the proliferation of normal human ovarian surface epithelium cells are inconclusive with a number of studies showing no effect ${ }^{80}$. Importantly, the gonadotropin theory has not been adapted to account for a fallopian tube site of origin. An in vitro study of tubal epithelial cell cultures did not display increased cellular proliferation or DNA damage in response to $\mathrm{LH}$ or $\mathrm{FSH}^{81}$.

\subsubsection{Androgen/Progesterone Hypothesis}

The androgen/progesterone hypothesis was proposed by Risch in 1998 and implicates raised levels of endogenous androgens in the pathogenesis of ovarian cancer, whereas progesterone may be protective against ovarian cancer development ${ }^{82}$. Several experimental lines of evidence indicate that androgens can increase proliferation and migratory activity of both malignant and normal ovarian cell lines ${ }^{83,84}$. More recent data suggest that the fallopian tube epithelium also expresses androgen receptors and displays increased cellular viability, proliferation and invasion in response to androgen exposure ${ }^{85}$. Epidemiologic associations between conditions characterized by high androgenic exposure and ovarian cancer risk also lend support to this model. Post-menopausal status, high body mass index (BMI) and possibly polycystic ovarian syndrome have been related to modestly increased risk of ovarian cancer in the general population ${ }^{86,87}$. Conversely, the protective effect of oral contraceptive use may be mediated by suppression of ovarian testosterone synthesis ${ }^{82}$. 
The progesterone hypothesis emerged from observations that the protective effect of pregnancy exceeds the extent of ovulation suppression alone, and that progestin-only oral contraceptives, which do not suppress ovulation, were associated with a comparable decrease in ovarian cancer risk to combined oral contraceptives ${ }^{82}$. Risch suggested that eight to nine months of elevated blood progesterone may be responsible for the additional ovarian cancer risk reduction seen with pregnancy. Furthermore, the protection gained for users of the progestin-only pill may be due to long-term exposure to potent synthetic progestins, compensating for the decrease in endogenous progesterone synthesis ${ }^{82}$.

Expression of progesterone receptors has been documented in the normal fallopian tube epithelium and ovarian surface epithelium independent of $B R C A$ mutation status ${ }^{88,89}$. Antiproliferative and pro-apoptotic effects of progestogens have been demonstrated in preclinical studies ${ }^{90}$. Progesterone is shown to inhibit cell proliferation and induce apoptosis by p53 upregulation in human ovarian carcinoma cell lines ${ }^{91}$. Three-year administration of progestincontaining oral contraceptives was associated with a four- to six-fold increase in the number of apoptotic ovarian epithelial cells compared to the control or estrogen-treated primates ${ }^{92}$. In vitro experiments also support a necroptotic effect of progesterone in p53-deficient murine fallopian tube epithelial cells ${ }^{93}$. These data support the hypothesis that high levels of progestogen may lead to 'clearing' of genetically damaged cells of the ovary or fallopian tube. This theory was evaluated a clinical trial of 64 high-risk women randomized to four to six weeks of oral levonorgestrel (LNG) or placebo ${ }^{94}$. Karyometric results revealed that LNG exposure shifted the nuclear signature of both ovarian and fallopian tube epithelial samples toward normal, resembling that of women at baseline risk ${ }^{94}$. Interestingly, there was no difference in the primary endpoints of apoptosis or TGF- $\beta$ signalling, which conflicts with findings from animal studies. This may indicate that progestin-mediated clearance occurs through non-apoptotic pathways in humans; however, the study was also limited by the heterogeneity of tubal specimens available for examination. Only $50 \%$ of fallopian tube sections contained the fimbriated end, which may have impacted the ability to detect a biological effect ${ }^{94}$.

Distinct from these studies, other evidence point toward a carcinogenic effect of progestogen exposure $^{95}$. At low concentrations, progesterone treatment may increase proliferation and 
decrease apoptosis of ovarian cancer cells ${ }^{96}$. A large clinical study of premenopausal $B R C A$ mutation carriers also observed that average luteal phase progesterone levels were $121 \%$ higher in carriers compared to noncarriers, which may contribute toward excess ovarian cancer risk ${ }^{97}$. In line with this theory, Kim et al. found that progesterone induced the development of ovarian cancer in a mouse model of HGSC, whereas pharmacologic blockade and genetic ablation of the PR inhibited HGSC development and metastasis formation ${ }^{98}$. Certainly more evidence is necessary to ascertain the biological mechanisms of progestogen action in ovarian cancer. 


\subsubsection{Management of Ovarian Cancer Risk in BRCA Mutation Carriers}

Various international guidelines and recommendations inform the clinical management of individuals who harbour a pathogenic BRCA variant ${ }^{99-102}$. BRCA1 and BRCA2 mutation carriers are characterized by an increased lifetime risk of breast and ovarian cancer and earlier onset of disease compared to the general population. The penetrance and epidemiology of hereditary breast and ovarian cancer syndrome is detailed in Section 1.1.1. Specific recommendations for ovarian cancer risk management including risk-reducing surgery, screening and modifiable risk factors will be reviewed.

\subsubsection{Surgical Prevention}

For unaffected individuals who are identified with a pathogenic $B R C A$ mutation, risk-reducing bilateral salpingo-oophorectomy (RRBSO) is the current standard of care for the management of ovarian cancer risk. RRBSO is a minimally invasive, laparoscopic procedure that removes the ovaries and fallopian tubes in their entirety ${ }^{103}$. There is substantial data demonstrating the effectiveness of RRBSO in a high-risk population with estimated ovarian cancer risk reductions ranging from $80 \%$ to $96 \%{ }^{39,104,105}$. A meta-analysis of 10 studies among BRCA mutation carriers confirmed approximately $80 \%$ reduction in risk of ovarian or fallopian tube cancer following RRBSO $^{106}$. Furthermore, in a Cochrane meta-analysis of 10 cohort studies of RRBSO in BRCA mutation carriers, the procedure was associated with a $94 \%$ reduction in ovarian-cancer specific mortality and a $68 \%$ reduction in all-cause mortality ${ }^{107}$.

Optimal timing of RRBSO must weigh the age-related risk of ovarian cancer against the adverse effects of early surgical menopause. While cumulative ovarian cancer risk increases with age, premature menopause is associated with a host of short-term and long-term morbidities, including cardiovascular, bone and cognitive decline ${ }^{103}$. Recommendations for surgical timing also vary by $B R C A$ mutation type. For $B R C A 1$ mutation carriers, the National Comprehensive Cancer Network Guidelines recommend RRBSO between 35 to 40 years and after the completion of childbearing ${ }^{99}$. Indeed, the risk of ovarian cancer is remote before the age of 30 , but approximately $26 \%$ of women with a BRCA1 mutation will develop ovarian cancer by the age of 50 years ${ }^{12,108}$. Since the onset of ovarian cancer occurs on average eight to 10 years later 
in $B R C A 2$ mutation carriers compared to BRCA1 mutation carriers, it is reasonable to delay RRBSO until age 40 to 45 in these women ${ }^{109}$. Currently, the uptake of RRBSO stands around $70 \%$ of women with a $B R C A$ mutation aged 35 and above ${ }^{110}$.

In view of the evidence that most HGSCs arise from the fallopian tube, bilateral salpingectomy with delayed oophorectomy has been proposed as an alternative to RRBSO. This is an appealing strategy for premenopausal women who wish to preserve fertility and avoid premature menopause. The procedure involves removal of the fallopian tubes with completion of oophorectomy closer to the time of natural menopause. Despite preliminary data suggesting safety and feasibility, there is no evidence of risk reduction or mortality benefit achieved after salpingectomy alone in high-risk patients ${ }^{99}$. Based on consensus from multiple international authorities, salpingectomy is not recommended as primary prevention in carriers of a $B R C A$ mutation at this time ${ }^{99,100,102}$. Prospective, multicentre trials to evaluate this question are ongoing (NCT02321228; NCT02760849).

\subsubsection{Screening}

RRBSO is the only proven intervention to the reduce ovarian cancer incidence and mortality, and ovarian cancer screening remains of limited value in the management of high-risk women. Proposed routine screening consists of transvaginal ultrasound combined with measures of serum cancer antigen 125 (CA125) every six months beginning at the age of $30^{99}$. Screening may be considered at clinician's discretion until the target age of RRBSO; however, there is no evidence that current screening modalities and regimens reduce ovarian cancer-specific mortality in highrisk populations. Large population-based studies of high-risk women monitored by transvaginal ultrasonography and CA125 showed improvements in early-stage detection of cases, but the effect on survival is unknown ${ }^{111,112}$.

\subsubsection{Modifiers of Ovarian Cancer Risk in BRCA Mutation Carriers}

Inherited mutations in $B R C A 1$ and $B R C A 2$ confer a significantly increased risk of breast and ovarian cancer. Nonetheless, there is observed interindividual variability in cancer risk among multi-generation families with mutations in the same gene, suggesting a role for non-genetic risk factors that modulate cancer penetrance in $B R C A$ mutation carriers ${ }^{113,114}$. Indeed, predisposition 
to developing breast or ovarian cancer is multifactorial, and novel risk prediction models incorporate the effects of gene mutation, family history, lifestyle and hormonal factors that modify risk for mutation carriers ${ }^{115}$. The following section evaluates the role of select nongenetic risk factors of $B R C A$-associated ovarian cancer, including reproductive, hormonal and lifestyle exposures.

\section{Reproductive Factors}

The characteristics related to reproduction and menstruation, including breastfeeding, parity and the timing of menarche or menopause are known to influence ovarian carcinogenesis ${ }^{116}$. The underlying mechanisms by which these factors protect against ovarian cancer development may involve reducing a woman's lifetime number of ovulations with concomitant changes in hormonal and inflammatory exposures (discussed in Section 1.1.6).

Breastfeeding is associated with a decreased risk of sporadic and hereditary ovarian cancer risk. A pooled analysis of 13 case-control studies concluded that breastfeeding reduces the risk of developing ovarian cancer by $24 \%$ compared to women who never breastfed (odds ratio $[\mathrm{OR}]=0.7695 \% \mathrm{CI} 0.71-0.80)^{117}$. These findings have been replicated in several large, high-risk cohorts $^{75,79,118}$. The largest evaluation of breastfeeding and ovarian cancer risk among 4,352 $B R C A 1$ and BRCA2 mutation carriers reported that a history of breastfeeding is associated with $23 \%$ risk reduction $(\mathrm{OR}=0.77 ; 95 \% \mathrm{CI}$ 0.66-0.90), which increased with longer durations of breastfeeding and did not vary by BRCA gene ${ }^{118}$.

Parity is an established protective factor for ovarian cancer in the general population, associated with $30 \%$ to $40 \%$ decreased risk compared to nulliparity ${ }^{119}$; however, the relationship is controversial among carriers. A 2014 meta-analysis analyzed five studies reporting on the association between timing and number of pregnancies and ovarian cancer risk in $B R C A$ mutation carriers ${ }^{116}$. Compared to nulliparous women, there was a significant decreased risk associated with four or more live births (effect size=0.42; 95\% CI 0.20-0.88) among BRCA1 mutation carriers alone. Pregnancy at older age did not provide stronger protection than pregnancy at younger age, such that null results were seen with age at first live birth for both mutation types ${ }^{116}$. 
For women in the general population, later age at menarche has been associated with a small decrease in ovarian cancer risk ( $\geq 15$ years vs $\leq 11$ years: relative risk $[R R]=0.88 ; 95 \%$ CI 0.80 $0.97)^{120}$. Later age at menopause has also been associated with increased ovarian cancer risk ( $>52$ years vs $\leq 45$ years: hazard ratio $[\mathrm{HR}]=1.46 ; 95 \% \mathrm{CI} 1.06-1.99$ ), and these associations may vary by histologic subtype ${ }^{120-122}$. In contrast, studies among high-risk women report no relationship between age at menarche and ovarian cancer risk $75,79,123,124$. One matched casecontrol study investigated the role of age at menopause among women with a BRCAl mutation and observed an $18 \%$ increased risk of ovarian cancer per year of delayed menopause (OR=1.18; $95 \%$ CI $1.03-1.35)^{79}$.

\section{Hormonal Factors}

Several epidemiologic studies have examined the use of exogenous hormone agents such as oral contraception, fertility treatments and hormone replacement therapy in relation to ovarian cancer. These associations support the etiologic role of "incessant ovulation" and endogenous hormone levels in the development of ovarian cancer. Section 1.3 provides a detailed overview of the epidemiologic data regarding contraceptive use and ovarian cancer across observational studies in the general and high-risk population.

Hormonal replacement therapy (HRT) consists of estrogen-only or estrogen-progestin regimens prescribed to treat menopausal symptoms. Evidence from a meta-analysis of 52 epidemiologic studies indicates that HRT is associated with a minimal increase in ovarian cancer risk in the general population $(\mathrm{RR}=1.14 ; 95 \% \mathrm{CI} 1.10-1.19)^{125}$. The data on cancer risk associated with HRT use in high-risk women is limited and inconsistent ${ }^{126}$. Two studies of women with $B R C A$ mutations found no relationship between HRT use and ovarian cancer risk ${ }^{127,128}$. Although one case-control study of 1,073 Jewish Israeli $B R C A$ mutation carriers reported a significant increase in risk associated with HRT (OR=2.58; 95\% CI 1.64-4.06), the sample size was small and subgroup analyses were not conducted ${ }^{124}$.

Fertility drugs are ovarian-stimulating drugs that temporarily raise circulating levels of gonadotropins and steroid hormones during in vitro fertilization treatment. A Cochrane metaanalysis of 25 studies and 182,972 women established that there is no relationship between fertility drugs and ovarian cancer risk in the general population ${ }^{129}$. Likewise, a matched case- 
control study of 941 pairs of BRCA mutation carrier showed that the use of any fertility drug did not increase risk of developing ovarian cancer ${ }^{130}$.

\section{Lifestyle Factors}

Lifestyle factors such as body weight, smoking and dietary habits have been investigated in relation to ovarian cancer risk, but there is a paucity of data among women with a hereditary predisposition $^{131}$.

Studies that have addressed the role of smoking in ovarian cancer risk report a low increased risk of developing ovarian cancer overall, but associations vary widely by histologic subtype. A meta-analysis of 51 epidemiological studies found that smoking was associated with a $79 \%$ increased risk of mucinous ovarian cancer $(\mathrm{RR}=1.79 ; 95 \% \mathrm{CI} 1.60-2.00)$ and a $20 \%$ decreased risk in endometrioid $(\mathrm{RR}=0.81 ; 95 \% \mathrm{CI}$ 0.72-0.92) and clear-cell ovarian cancers $(\mathrm{RR}=0.80$; 95\% CI 0.65-0.97) compared to women who never smoked ${ }^{132}$. There was no relationship between smoking and serous ovarian cancer $(\mathrm{RR}=0.99 ; 95 \% \mathrm{CI} 0.93-1.06)^{132}$. In contrast, Ko et al. conducted a prospective cohort study among 15,731 BRCA mutation carriers and observed a 1.7-fold increased risk of ovarian cancer overall among ever-smokers compared to neversmokers $\left(\mathrm{HR}=1.68 ; 95 \%\right.$ CI 1.06-2.67), which did not vary by BRCA gene ${ }^{133}$.

Systematic reviews of the general population indicate that BMI is associated with modestly increase ovarian cancer risk ${ }^{87,134}$. The literature is limited among high-risk women. Qian et al. conducted the largest study in BRCA mutation carriers, applying a Mendelian randomization approach to examine the relationship between BMI and ovarian cancer risk ${ }^{135}$. Higher adult BMI was associated with significantly increased risk in premenopausal women (HR=1.25; 95\% CI 1.06-1.48) but not postmenopausal women ( $\mathrm{HR}=0.98$; 95\% CI 0.88-1.10). A recent prospective evaluation of 4,340 BRCA mutation carriers also suggested that adult weight gain of more than $20 \mathrm{~kg}$ is associated with a two-fold increased risk of $B R C A$-associated ovarian cancer compared to women who maintained stable weight $(\mathrm{HR}=2.00 ; 95 \% \mathrm{CI} 1.13-3.54)^{136}$.

Dietary factors such as coffee consumption have generated conflicting findings with respect to ovarian cancer risk. A recent meta-analysis of 20 case-control studies found no significant association between total coffee intake, total caffeine intake or caffeinated coffee intake and risk 
of ovarian cancer in the general population ${ }^{137}$. Interestingly, there was a significant inverse association between decaffeinated coffee intake and risk $(\mathrm{OR}=0.72 ; 95 \% \mathrm{CI}$ 0.58-0.90).

Similarly, data from high-risk populations indicate no association between coffee consumption and risk of ovarian cancer $(\mathrm{OR}=0.7 ; 95 \% \text { CI } 0.4-1.3)^{123}$.

Table 1. Summary of known and potential modifiers of sporadic and $B R C A$-associated ovarian cancer risk

\begin{tabular}{|c|c|c|}
\hline Risk modifier & Sporadic ovarian cancer risk & $B R C A$-associated ovarian cancer risk \\
\hline \multicolumn{3}{|l|}{ Reproductive factors } \\
\hline Breastfeeding & $\downarrow$ & $\downarrow$ \\
\hline Parity & $\downarrow$ & $\downarrow$ \\
\hline Later age at menarche & $\downarrow$ & - \\
\hline Later age at menopause & $\uparrow$ & $\uparrow$ \\
\hline \multicolumn{3}{|l|}{ Hormonal factors } \\
\hline Oral contraceptives & $\downarrow$ & $\downarrow$ \\
\hline Fertility treatment & - & - \\
\hline Hormone replacement therapy & $\uparrow$ & $?$ \\
\hline \multicolumn{3}{|l|}{ Lifestyle factors } \\
\hline Body weight & $\uparrow$ & ? \\
\hline Smoking & $\uparrow$ & $\uparrow$ \\
\hline Coffee & - & - \\
\hline
\end{tabular}

Legend: $\downarrow=$ decreased ovarian cancer risk; $\uparrow=$ increased ovarian cancer risk; ? = unknown association with ovarian cancer risk; $-=$ no association with ovarian cancer risk 


\subsection{Contraception}

\subsubsection{The Hypothalamic-Pituitary-Ovarian Axis and Regulation of the Ovarian Cycle}

The hypothalamic-pituitary-ovarian axis refers to the cumulative effects of the hypothalamus, pituitary gland and ovaries in the development and function of the female reproductive system. Low levels of ovarian steroids prior to the onset of menstruation stimulate the pulsatile release of the neuropeptide, gonadotrophin-releasing hormone $(\mathrm{GnRH})$ from the hypothalamus (Figure $2)^{138}$. GnRH stimulates the synthesis and release of gonadotropins from the anterior pituitary gland, namely luteinizing hormone ( $\mathrm{LH}$ ) and follicle stimulating hormone (FSH). According to the two-cell two-gonadotrophin model, estradiol synthesis requires the LH-dependent production of androgens by theca cells. In response to blood-borne FSH, granulosa cells upregulate expression of the enzyme, aromatase, which is required to convert androgens from adjacent theca cells into estradiol, serving as the primary source of elevated estrogen during the follicular phase of the menstrual cycle. FSH from the pituitary gland is also important for granulosa cell proliferation and follicular development ${ }^{138}$.

Estradiol plays a key role in regulating gonadotropin secretion through both negative and positive feedback loops depending on the time in the cycle. During the mid-follicular phase, low levels of estradiol exert a negative feedback effect at the level of the hypothalamus and pituitary to suppress levels of LH and FSH. During the late follicular phase, high sustained levels of estradiol coming from preovulatory follicles induces a positive feedback effect, increasing the pulsatile release of GnRH and enhanced LH and FSH production. This culminates to the midcycle LH surge, which develops over approximately 50 hours and triggers ovulation of the oocyte from the mature follicle ${ }^{138}$. The remaining granulosa and theca cells of the follicle form the corpus luteum, a transient endocrine structure that produces peak levels of progesterone during the luteal phase of the menstrual cycle. High levels of progesterone exert negative feedback on gonadotropin secretion, evident in low levels of LH and FSH. The corpus luteum eventually degrades to initiate the next follicular phase. 


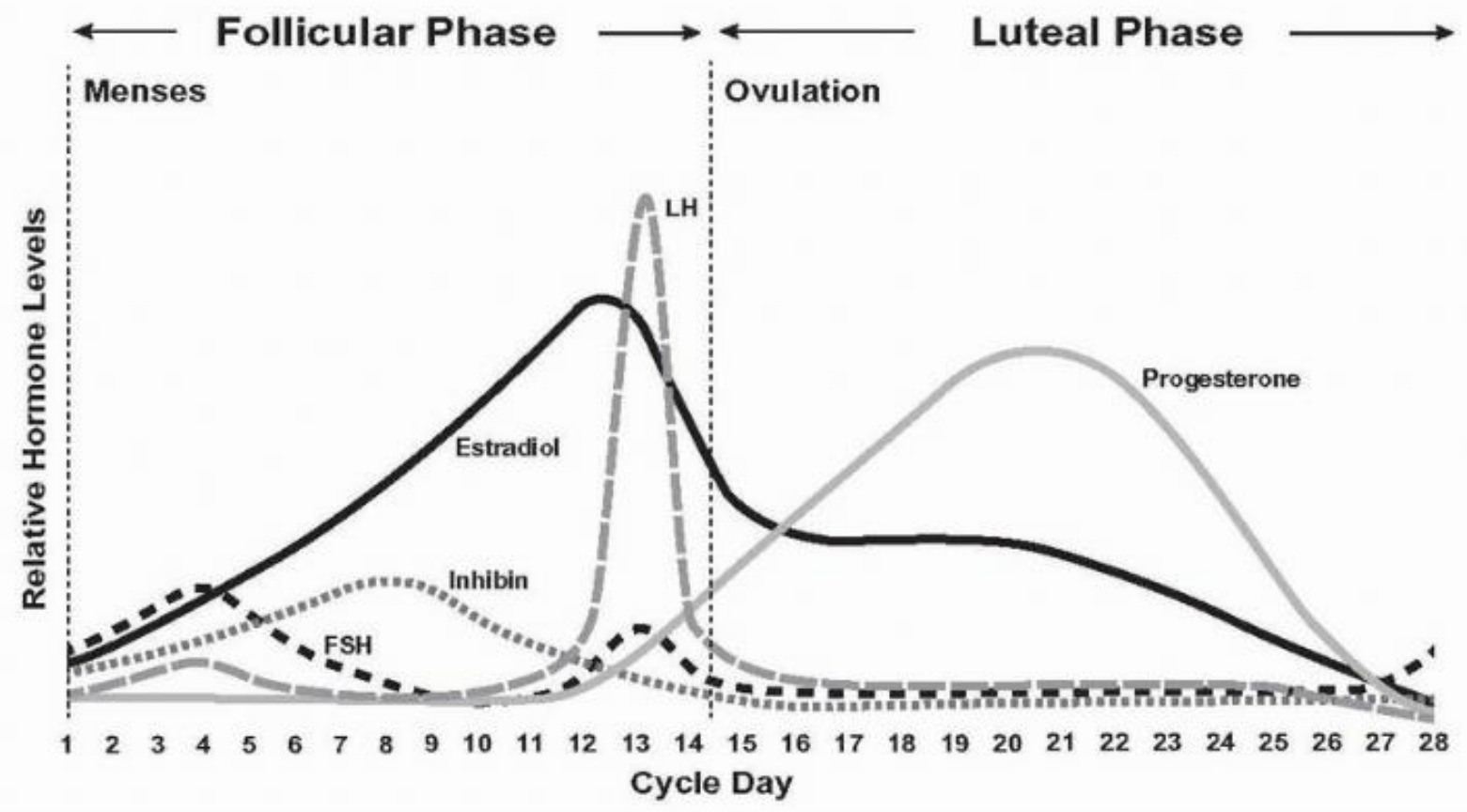

Figure 2. Hormonal regulation of the menstrual cycle

Footnote to 


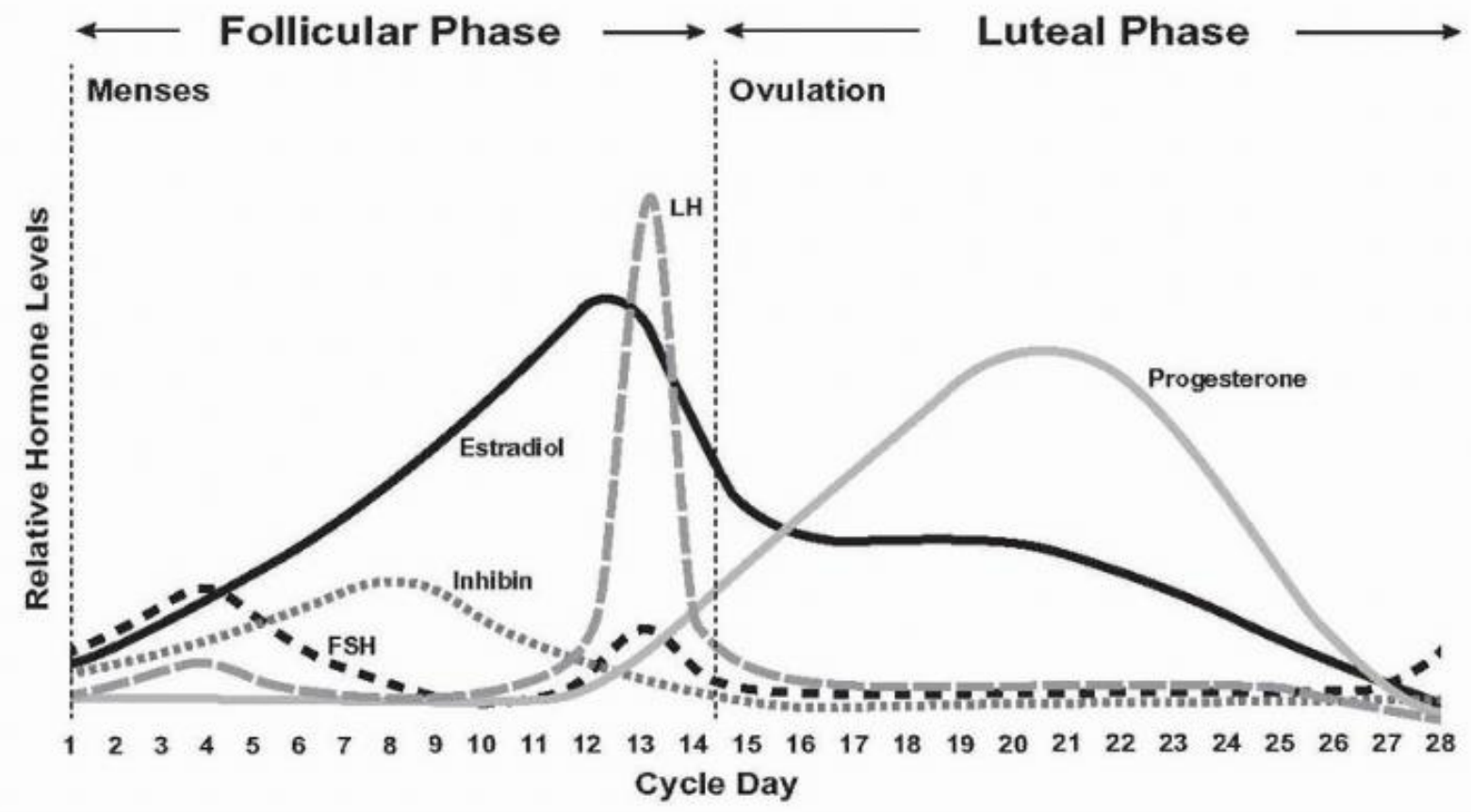

Figure 2: The early follicular phase of the menstrual cycle is characterised by low levels of the steroid hormones, estrogen and progesterone, corresponding to a slight increase in follicle stimulating hormone (FSH). FSH drops by the mid-follicular phase due to rising levels of estradiol and inhibin from the developing follicle. At the peak of follicular development, high levels of blood estradiol positively induce the release of luteinizing hormone (LH). The LH surge triggers ovulation and the formation of the corpus luteum. During the luteal phase, the corpus luteum secretes estrogen and progesterone. If fertilization does not occur, the corpus luteum degenerates and the drop in progesterone initiates menstruation.

Reproduced from Jensen JT, Creinin MD. Speroff \& Darney's Clinical Guide to Contraception. Sixth edition. Philadelphia, PA: Lippincott Williams \& Wilkins/Wolters Kluwer; 2020 by permission from Wolters Kluwer Health, Inc. 


\subsubsection{Contraceptive Pharmacology}

\subsubsection{Classification of Estrogens and Progestogens}

Estrogens are a family of natural and synthetic hormones with activity at estrogen receptors. There are four endogenous estrogens that differ by the number of hydroxy groups present on the 19-carbon backbone: estrone, estradiol, estriol and estetrol. Progestogens are defined as natural and synthetic hormones that bind the progesterone receptor (PR) and act as agonists. Among various parameters, progestational activity is evaluated in vivo by the ability to induce secretory changes in an estrogen-primed endometrium ${ }^{139}$. On the other hand, progestogens can exert agonistic, partial agonistic and antagonistic effects at other steroid receptors, including estrogen receptor, androgen receptor, glucocorticoid receptor and mineralocorticoid receptor ${ }^{140}$. Progestational effect is dependent on the synergistic action of endogenous and exogenous estrogens that induce $\mathrm{PR}^{141}$. The 21-carbon compound, progesterone is the only naturallyoccurring progestogen that is critical for female reproductive function and capable of sustaining pregnancy.

Natural estrogens and progesterone have low bioavailability when administered orally due to rapid metabolism in the gastrointestinal tract and liver. Synthetic hormones have been developed by chemically modifying the steroid skeleton to sterically hinder the action of metabolizing enzymes. As a result, synthetic estrogens and progestogens have more favourable bioavailability and half-life following oral administration ${ }^{142}$.

Ethinyl estradiol (EE) and its prodrug, mestranol are synthetic estrogens used in combined oral contraception. First generation combined oral contraceptives contained mestranol, the 3-methyl ether of EE, which is rapidly demethylated to $\mathrm{EE}$ in the gastrointestinal tract and liver following oral administration ${ }^{143}$. Animal studies indicate that mestranol is weaker than EE, such that $50 \mathrm{mg}$ of mestranol is pharmacokinetically bioequivalent to $35 \mathrm{mg}$ of EE. Mestranol is rarely used in combined oral contraceptives today, and nearly all modern combined oral contraceptives contain $\mathrm{EE}^{144}$. EE is a very potent oral estrogen. Unlike estradiol, the addition of an ethinyl group at the 17 -carbon position prevents oxidization by $17 \beta$-estradioldehydrogenase, resulting in increased oral activity ${ }^{141}$. After ingestion, EE is rapidly absorbed in the gastrointestinal tract. First-pass 
metabolism at the gut mucosa and liver renders a bioavailability of $38 \%$ to $48 \%{ }^{141}$. Circulating EE predominantly exists as a 3-sulphate that is bound almost exclusively to albumin ${ }^{141}$. There is large intraindividual and interindividual variability in blood EE levels due to variations in metabolic capacity. Serum concentrations of EE peak one to two hours following oral intake and typically represent $1 \%$ of the ingested dose ${ }^{141}$.

Synthetic progestogens, called progestins, are used therapeutically for contraception and hormone replacement therapy due to their anti-ovulatory and anti-estrogenic effects ${ }^{141}$. Progestins can be classified based on their chemical structure, which varies widely between molecules and results in different patterns of hormonal activity (Table 2) ${ }^{139}$. Progestins that are structurally related to progesterone are further divided into the pregnane and 19-norgpregnane derivatives. Progestins that are structurally related to testosterone are classified as estranes or gonanes. The classification scheme and names of progestins in each of the categories is summarized in Table 2.

Table 2. Classification scheme of progestins used in hormonal contraception

\begin{tabular}{|c|c|c|c|c|c|}
\hline $\begin{array}{l}\text { Parent } \\
\text { hormone }\end{array}$ & \multicolumn{2}{|l|}{ Testosterone } & \multicolumn{2}{|l|}{ Progesterone } & Spironolactone \\
\hline Derivative & \multicolumn{2}{|c|}{ 19-Nortestosterone } & \multicolumn{2}{|c|}{ 17a-hydroxyprogesterone } & $17 \alpha-$ \\
\hline Class & Gonanes & Estranes & Pregnanes & Norpregnanes & Spironolactone \\
\hline Progestin & $\begin{array}{l}\text { Norgestrel } \\
\text { Norgestimate } \\
\text { Desogestrel } \\
\text { Gestodene } \\
\text { Levonorgestrel }\end{array}$ & $\begin{array}{l}\text { Norethindrone } \\
\text { (norethisterone) } \\
\text { Norethindrone } \\
\text { acetate } \\
\text { Ethinodiol } \\
\text { diacetate } \\
\text { Norethynodrel } \\
\text { Lynestrol }\end{array}$ & $\begin{array}{l}\text { Medroxyprogesterone } \\
\text { acetate } \\
\text { Megestrol acetate } \\
\text { Chlormadinone acetate } \\
\text { Cyproterone acetate } \\
\text { Dydrogestrone } \\
\text { Medrogesterone }\end{array}$ & $\begin{array}{l}\text { Demegestone } \\
\text { Promegestone } \\
\text { Trimegestone } \\
\text { Nomegestrol } \\
\text { acetate } \\
\text { Nesterone }\end{array}$ & Drospirenone \\
\hline
\end{tabular}

\subsubsection{Testosterone Derivatives}

The most common contraceptive progestins belong to the 19-nortestosterone class, which are subdivided into estrane and gonane families based on the structure of the carbon ring from which the progestin is derived. All 19-nortestosterones are derivatives of testosterone, but exert major hormonal effect at PRs with minimal androgenic and anabolic properties ${ }^{145}$. 
The estrane family (C18) is characterised by an 18-carbon parent steroid with a methyl group substitution at the 13-carbon position. These include commonly used progestins such as norethindrone, norethindrone acetate and ethynodiol diacetate. Following oral administration, all estranes are rapidly metabolized to the progestationally active compound, norethindrone. In addition to potent progestational activity, all 19-nortestosterone derivatives have low androgenic and estrogenic activity ${ }^{145}$.

13-ethylgonanes, otherwise known as gonanes (C17) possess an ethyl group at the 13-carbon position, which confers greater progestational and less androgenic activity in vitro ${ }^{138}$. While all estranes have the same activity as norethindrone, individual gonanes have distinct properties. Among the most commonly used gonanes include LNG, which is the hormonally active isomer

of the racemic compound, norgestrel. Synthesized in 1966, LNG is one of the most potent orally active progestogens ${ }^{146}$. LNG binds PR with high affinity and strongly inhibits gonadotrophin secretion. LNG also exerts relatively strong androgenic activity and anti-estrogenic activity without glucocorticoid, mineralcorticoid or anti-mineralocorticoid potential. It does not undergo significant hepatic first-pass metabolism, thus bioavailability is virtually $100 \%$ after oral administration. LNG is hydrolyzed by the liver and eliminated after conjugation with glucuronic $\operatorname{acid}^{147}$. Newer, third-generation progestogens in the gonane family include desogestrel, norgestimate and gestodene that offer further reduced androgenic activity ${ }^{144}$. Desogestrel and norgestimate are prodrugs that are converted in vivo to the active metabolites etonogestrel and levonorgestrel, respectively ${ }^{146}$. In addition to low androgenic activity, desogestrel exhibits high antigonadotropic activity ${ }^{147}$. Gestodene has inherent progestational activity, slight mineralocorticoid activity and high anti-estrogenic activity ${ }^{148}$. Gestodene is characterized by prolonged half-life and $100 \%$ oral bioavailability ${ }^{147}$. Several combined oral contraceptives containing gestodene and EE are available, including Logest and Minulet.

\subsubsection{Progesterone Derivatives}

Progestins derived from progesterone are categorized into two families that differ at the 10carbon position. The pregnane class of progestins possesses a methyl group at the 10-carbon location and include medroxyprogesterone acetate, megestrol acetate, chlormadinone acetate, cyproterone acetate, dydrogesterone and medogestone. Medroxyprogesterone acetate (MPA) 
exhibits relatively high progestational activity and is typically administered as a $150 \mathrm{mg}$ injection of depot MPA (DMPA; Depo-Provera). The 19-norpregnanes (norpregnanes) are progesterone derivatives that lack a methyl group at the carbon 10 position. The hormonal pattern of norpregnanes is similar to that of pregnanes, in terms of selective binding to PR with no androgenic, estrogenic or glucocorticoid activity ${ }^{149}$.

\subsubsection{Spironolactones}

Drospirenone is a derivative of $17 \alpha$-spironolactone that has been available in combination with EE (Yasmin) for oral contraception since 2001. This progestin has strong anti-mineralocorticoid and anti-androgenic activity without androgenic, estrogenic, glucocorticoid or antiglucocorticoid effects ${ }^{147,150}$.

\subsubsection{Molecular Mechanism of Action of Progestogens}

Progestogens exert their biological effects via interaction with steroid receptors, which are intracellular ligand-activated transcription factors that regulate the transcription of downstream target genes. The progestational activity of all progestins is due to their relatively high binding affinity progesterone receptors A and B (PRA and PRB). Binding of progestogen to PR causes a conformational change, such that the receptor-steroid complex dimerizes, translocates to the nucleus and initiates transcription of target genes ${ }^{150}$. This interaction to its receptor may be agonistic or antagonistic to increase or decrease transcription of target genes. Generally, PRAs act as transcriptional repressors and PRBs act as transcriptional activators ${ }^{151}$.

Progestins vary in chemical structure which accounts for differences in their affinity for other members of the steroid receptor family, including androgen receptor, glucocorticoid receptor and mineralocorticoid receptor. Most progestins demonstrate minimal to no activity at estrogen receptors ${ }^{145}$. The hormonal activities of various progestogens are summarized in Table 3 . 
Table 3. Biological activities of various progestogens

\begin{tabular}{|c|c|c|c|c|c|c|c|}
\hline \multirow[b]{2}{*}{ Progestogen } & \multirow[b]{2}{*}{$\begin{array}{c}\text { Anti- } \\
\text { gonadotrophic }\end{array}$} & \multicolumn{2}{|c|}{ Estrogenic } & \multicolumn{2}{|c|}{ Androgenic } & \multirow[b]{2}{*}{$\begin{array}{l}\text { Gluco } \\
\text { corticoid }\end{array}$} & \multirow{2}{*}{$\begin{array}{c}\text { Anti } \\
\text { mineralo } \\
\text { corticoid }\end{array}$} \\
\hline & & Pro & Anti & Pro & Anti & & \\
\hline Progesterone & + & - & + & - & + & \pm & \pm \\
\hline \multicolumn{8}{|c|}{ Pregnane derivatives: non acetylated } \\
\hline Dydrogestrone & - & - & + & - & \pm & - & \pm \\
\hline Medrogesterone & + & - & + & - & \pm & & \\
\hline \multicolumn{8}{|c|}{ Pregnane derivatives: acetylated } \\
\hline $\begin{array}{l}\text { Medroxyprogesterone } \\
\text { acetate }\end{array}$ & + & - & + & \pm & - & + & - \\
\hline Megestrol acetate & + & - & + & \pm & + & + & - \\
\hline Chlormadinone acetate & + & - & + & - & + & + & - \\
\hline Cyproterone acetate & + & - & + & - & ++ & + & - \\
\hline \multicolumn{8}{|c|}{ 19-Norpregnane derivatives: non acetylated } \\
\hline Demegestone & + & - & + & - & - & - & - \\
\hline Promegestone & + & - & + & - & - & - & - \\
\hline Trimegestone & + & - & + & - & \pm & - & \pm \\
\hline
\end{tabular}

19-Norpregnane derivatives: acetylated

\begin{tabular}{l|c|c|c|c|c|c|c}
\hline Nomegestrol acetate & + & - & + & + & \pm & - & - \\
\hline Nesterone & + & - & + & - & - & - & - \\
\hline
\end{tabular}

\section{9-Nortestosterone derivatives: estranes}

\begin{tabular}{l|c|c|c|c|c|c|c}
\hline $\begin{array}{l}\text { Norethisterone } \\
\text { (Norethindrone) }\end{array}$ & + & + & + & + & - & - & - \\
\hline Norethisterone acetate & + & + & + & + & - & - & - \\
\hline Norethynodrel & + & + & \pm & \pm & - & - & - \\
\hline Lynestrenol & + & + & + & + & - & - & - \\
\hline Tibolone (metabolites) & + & + & - & + & - & - & - \\
\hline Dienogest & + & \pm & + & - & + & - & - \\
\hline
\end{tabular}

19-Nortestosterone derivatives: gonanes

\begin{tabular}{l|c|c|c|c|c|c|c}
\hline Levonorgestrel & + & - & + & + & - & - & - \\
\hline Norgestimate & + & - & + & + & - & - & - \\
\hline Desogestrel (etogestrel) & + & - & + & + & - & - & - \\
\hline Gestodene & + & - & + & + & - & + & + \\
\hline
\end{tabular}

\section{Spironolactone derivative}

Drospirenone

Legend: $++=$ Strongly positive $;+=$ Positive $+ \pm=$ Weakly positive; $-=$ Negative

Reproduced from Schindler AE. Pharmacology of Progestogens. In: Progestogens in Obstetrics and Gynecology. Second edition. Springer International Publishing; 2021 by permission from Springer Nature. 


\subsubsection{Overview of Contraceptive Methods}

\subsubsection{Combined Oral Contraceptives}

Combined oral contraceptives contain two synthetic steroid components, an estrogen and a progestin. The perfect-use and typical-use failure rates are $0.3 \%$ and $9 \%$ respectively ${ }^{152}$. Oral contraceptives are used by at least 150 million women worldwide and account for $16 \%$ of contraceptive use among women of reproductive age (15-49 years) ${ }^{153}$. Oral contraceptives were first approved in 1960 by the United States Food and Drug Administration. Early formulations contained upwards of $150 \mu \mathrm{g}$ of EE per tablet, but mean estrogen doses have declined over time to improve the safety profile without sacrificing contraceptive efficacy ${ }^{152}$. Most pills in the 1960s and 1970s contained $50 \mu \mathrm{g}$ of $\mathrm{EE}^{154}$. By 1988, 80\% of oral contraceptives in the United States contained less than $50 \mu \mathrm{g}$ of $\mathrm{EE}$, and the majority of formulations available today contain $20 \mu \mathrm{g}$ to $35 \mu \mathrm{g}$ of $\mathrm{EE}^{154,155}$. Concentrations of progestins have also decreased five-fold since the 1960s to under than $100 \mu \mathrm{g}$ today ${ }^{140}$. Alongside reductions in hormonal dose, new progestin types have changed over the years (see Section 1.2.2). Data from the National Survey of Family Growth from 2012 recorded nearly 90 unique formulations of oral contraceptives used in the United States alone ${ }^{156}$. Although prescribing patterns differ by country, most oral contraceptive users adopted formulations containing EE in combination with LNG, norgestimate, norethindrone, or drospirenone ${ }^{155-157}$.

The primary mechanism of action of the combined oral contraceptive pill is inhibition of ovulation. The progestational agent primarily inhibits $\mathrm{LH}$ secretion via negative regulation at the hypothalamus and anterior pituitary gland, which terminates the preovulatory LH surge. The estrogenic component suppresses FSH secretion and the emergence of a dominant follicle. These effects are reflected in the reduction of plasma pituitary gonadotropins to levels incompatible with ovulation among combined oral contraceptive users ${ }^{158,159}$. Moreover, when equal doses of $\mathrm{EE}$ are orally administered in combination with different progestins, greater suppression of serum LH and FSH is observed with the more potent progestin ${ }^{160}$.

The estrogen also potentiates the action of the progestin by increasing the concentration of intracellular PRs, which has allowed the reduction of progestin dose over the past decades ${ }^{144}$. It is important to note that follicular growth is seen with low-dose combination pills; however, 
these follicles do not mature and the progestin component is sufficient block the LH surge that is necessary for ovulation.

The effect of the progestational agent takes precedence over the estrogen, such that the effects of oral contraception at the lower genital tract reflect progestational stimulation ${ }^{138}$. The progestin in the oral contraceptive pill causes thickening of cervical mucus to be impervious to sperm transport. In addition, endometrial decidualization and atrophy in a progestin-dominant environment impairs implantation of the fertilized ovum. Progestational influence on secretion and peristalsis within the fallopian tubes provides additional contraceptive effect. These secondary mechanisms alone afford effective contraception independent of ovulation inhibition.

\subsubsection{Long-acting Reversible Contraceptives}

In addition to oral administration, various injectable, implantable and intrauterine devices (IUDs) have been developed over the years for effective, long-acting reversible contraception (LARC) that require no maintenance after insertion. These "set and forget" methods guarantee high user compliance, such that that "typical use" failure rates are very similar to failure rates associated with "perfect use"161. In fact, annual failure rates associated with LARC methods in the United States are less than $1 \%{ }^{162}$. LARC methods can be classified into those that provide systemic versus local delivery of progestin. Contraceptive implants and injections provide a continued release of progestin into the surrounding tissues that is absorbed into systemic circulation. IUDs provide continued local administration of LNG to the uterus to avoid systemic effects ${ }^{163}$. The three LARC methods are described below with an emphasis on historical development, epidemiology, basic pharmacology and mechanism of action.

\subsubsection{Implants}

Contraceptive implants consist of progestin-only subdermal rods or capsules inserted under the skin of the upper arm. Progestin diffuses in a sustained-release fashion from the implant into the interstitial fluid before absorption into systemic circulation. Similar to other LARCs, implants have higher contraceptive efficacy than oral or barrier methods with a $0.5 \%$ perfect-use and typical-use failure rate ${ }^{164}$. 
The Norplant LNG system was the first implant in widespread use. It was approved in Finland in 1983 and marketed in the United States and Canada by 1990 and 1994, respectively ${ }^{165}$. Norplant consisted of six capsules, each containing $36 \mathrm{mg}$ of crystalline LNG, effective for up to five years. Norplant has since been discontinued and superseded by Jadelle, capable of delivering the same daily dose of LNG utilizing only two rods (75 mg LNG each). Jadelle was first introduced in Finland in 1997 and now holds regulatory approval in numerous countries. Both Norplant and Jadelle contain $150 \mathrm{mg}$ LNG and are considered bioequivalent in terms of contraceptive efficacy and side-effect profile ${ }^{166}$. The LNG release rate is $100 \mu \mathrm{g} /$ day during the first few months of use, which decreases to $40 \mu \mathrm{g} /$ day by one year and $20 \mu \mathrm{g} /$ day by two years. This provides a continuous low dose of LNG that is equivalent to approximately $25 \%$ to $50 \%$ of the daily dose delivered by low-dose combined oral contraceptives in the first few months of use ${ }^{138,167}$. Blood levels of LNG peak around $772 \mathrm{pg} / \mathrm{mL}$ within two days and decline to $435 \mathrm{pg} / \mathrm{mL}$ within one month, $357 \mathrm{pg} / \mathrm{mL}$ at six months and $280 \mathrm{pg} / \mathrm{mL}$ sustained from three to five years ${ }^{167}$.

Implanon and Nexplanon are available as single rod-implant systems containing $68 \mathrm{mg}$ of etonogestrel and a duration of action of three years. Implanon was first marketed in 1998 in Indonesia and is now available in over 30 countries $^{168}$. Nexplanon, approved in the United States in 2011, exhibits comparable hormonal release rates to Implanon. This rate declines from 60 $\mu \mathrm{g} /$ day to $40 \mu \mathrm{g} /$ day of etonogestrel within the first 12 months to $30 \mu \mathrm{g} /$ day by two years ${ }^{138,169}$. Serum concentrations reach $266 \mathrm{pg} / \mathrm{mL}$ of etonogestrel within eight hours of insertion, which is sufficient to inhibit ovulation ${ }^{170}$. A peak plasma level of around $813 \mathrm{pg} / \mathrm{mL}$ etonogestrel is achieved by four days and declines to $196 \mathrm{pg} / \mathrm{mL}$ by one year and $156 \mathrm{pg} / \mathrm{mL}$ by three years $^{166,171}$.

Implants are highly effective contraceptives and three modes of action have been investigated. In clinical trials, measures of progesterone levels and ovarian ultrasound scanning have determined that etonogestrel contraceptive implants (Implanon, Nexplanon) consistently inhibit ovulation through a three-year period via suppression of the LH surge; however, ovarian follicular activity remains present with periodic peaks of serum estradiol. After one year of use, blood estradiol levels are about $50 \mathrm{pg} / \mathrm{mL}$ and increase to $100 \mathrm{pg} / \mathrm{mL}$ by the third year ${ }^{166}$. 
Increased cervical mucus viscosity and endometrial thinning were also documented among users $^{172}$.

In contrast, about one-third of all cycles are ovulatory among users of LNG implants (Norplant, Jadelle), which increases from $10 \%$ within the first year to over $50 \%$ by five years ${ }^{138}$. To maintain high contraceptive efficacy, the progestin agent confers additional local effects including thickening of the cervical mucus, which forms a barrier to sperm penetration. Further, the progestin induces endometrial atrophy, which prevents implantation in the event of a fertilized egg.

\subsubsection{Intrauterine Devices}

An IUD utilizes a polyethylene, T-shaped frame and releases either copper or a synthetic progestin into the uterine cavity for contraceptive action. The first and most widespread hormonal IUD is the $52 \mathrm{mg}$ levonorgestrel-bearing IUD (LNG IUD) with an effective lifespan of five years. After launching in Finland in 1990, the LNG IUD expanded across most European countries by the late 1990 s and was approved in the United States in $2001^{173}$. Now available in over 140 countries worldwide, the $52 \mathrm{mg}$ LNG IUD is commercially marketed under the brand names, Mirena, Levosert and Liletta ${ }^{174}$. Until the last decade, the IUD was not recommended for use in nulliparous women due to concerns about complications and difficulty of insertion. In light of mounting evidence supporting the safety and efficacy of IUD use in younger age groups, various health authorities now recommend IUDs as a first-line contraceptive option in nulliparous women and adolescents ${ }^{175}$.

Each device contains a progestin reservoir in the vertical stem that slowly releases hormone through a rate-limiting membrane. In the first year, the $52 \mathrm{mg}$ LNG IUD releases $20 \mu \mathrm{g}$ of LNG per day, which decreases to $10 \mathrm{ug}$ of LNG per day by five years ${ }^{176}$. The LNG IUD is also available in $19.5 \mathrm{mg}$ (Kyleena) and $13.5 \mathrm{mg}$ (Skyla/Jaydess) strengths that provide equally reliable contraception over a five-year and three-year lifespan, respectively ${ }^{177}$. The $19.5 \mathrm{mg}$ LNG IUD releases $17.5 \mathrm{ug}$ LNG daily during the first year, which declines to $7.4 \mathrm{ug} /$ day at five years $^{178}$. The $13.6 \mathrm{mg}$ LNG IUD has an initial release rate of about $14 \mathrm{ug} /$ day over the first year that declines to $5 \mathrm{ug} /$ day by three years ${ }^{179}$. Systemic LNG exposure achieved with use of an IUD 
is relatively low compared to that of LNG implants and oral contraceptives ${ }^{180}$. In a pooled analysis of pharmacokinetic data from phase II and phase III studies, the average serum LNG concentrations of the $13.5 \mathrm{mg}, 19.5 \mathrm{mg}$ and $52 \mathrm{mg} \mathrm{LNG} \mathrm{IUD} \mathrm{over} \mathrm{three} \mathrm{years} \mathrm{were} 74.3 \mathrm{ng} / \mathrm{L}$, $114 \mathrm{ng} / \mathrm{mL}$ and $218 \mathrm{ng} / \mathrm{mL}$, respectively ${ }^{177}$. Serum levels are subject to wide interindividual variation depending on body weight and sex hormone-binding globulin levels ${ }^{181,182}$.

Various inert and metal-coated IUDs have been available historically such as the Lippes Loop and stainless-steel IUDs that are widely used in China ${ }^{183}$. The most widely distributed nonhormonal IUD today is the copper-bearing IUD (ParaGard) that contains $200 \mathrm{~mm}^{2}$ to $380 \mathrm{~mm}^{2}$ of exposed copper wire to enhance spermicidal effect ${ }^{183}$. It was launched in the United States in 1988 and is approved for use for 10 to 12 years.

Until the 1980s, IUDs were widely misconceived as abortifacients that acted by preventing implantation of the fertilized egg or destroying existing embryos ${ }^{184}$. Today it is known that the principle contraceptive mechanism of action of the LNG IUD is inhibition of fertilization by preventing sperm-egg union. This occurs through induction of a weak foreign body reaction, suppression of endometrial growth, and increased viscosity of cervical mucus that inhibits sperm transport. The foreign body effect, induced by the presence of the IUD frame, is a local sterile inflammatory reaction that renders the uterine cavity toxic to sperm and ova ${ }^{185}$. It is characterized by the recruitment of leukocytes into the intrauterine space, including neutrophils, mononuclear cells and macrophages 1 . In tubal washings of IUD users compared to control cases, no sperm or fertilized ova were recovered, which was attributed to the phagocytic activity of excess white blood cells in the uterus ${ }^{187}$. Sperm may also be destroyed through the elevation of cytotoxic peptides that inhibit sperm motility, capacitation and survival ${ }^{188}$. Additional local contraceptive effects of the progestin agent include morphological changes at the endometrium that lower receptivity to implantation. Critchley et al. reported stromal decidualization and glandular atrophy in endometrial biopsies of women collected up to 12 months following LNG IUD insertion ${ }^{189}$. At the level of the cervix, LNG exposure increases the viscosity of cervical mucous to prevent passage of sperm into the uterus. This was supported by the absence of sperm transport through cervical mucus samples collected from LNG IUD users, as evaluated by the sperm cervical mucus penetration test ${ }^{190}$. 
The majority of women using the LNG IUD will have ovulatory cycles, as assessed by hormonal measurement and follicular ultrasonography ${ }^{191,192}$. Ovulation is suppressed in only $20 \%$ to $55 \%$ of users; however, the effect of the LNG IUD on ovarian function will depend on circulating levels of LNG, which can vary widely between individuals. With continued use, the hormonal release rate declines and the incidence of ovulation increases. By one year after insertion, twothirds of cycles are ovulatory ${ }^{193}$. By four years after insertion, almost $90 \%$ of menstrual cycles are ovulatory ${ }^{194}$. Early clinical trials of the LNG IUD suggest a daily release rate of $50 \mathrm{ug}$ LNG is necessary to inhibit ovulation, compared to modern devices that release $20 \mathrm{ug}$ LNG daily ${ }^{195}$. Because ovarian steroidogenesis is not affected, circulating estradiol levels also remain within the range of normal menstrual cycles $(100 \mathrm{pg} / \mathrm{mL} \text { to } 130 \mathrm{pg} / \mathrm{mL})^{196}$.

Similar to the hormonal IUD, the copper IUD inhibits fertilization by impeding sperm transport and the capacity of sperm to fertilize an ovum. All IUD types generate a foreign body reaction, but the inflammatory response is enhanced by the continuous release of copper ions into the luminal fluids of the genital tract ${ }^{197}$. In animal studies, the addition of copper stimulates greater mobilization of leukocytes into the uterine cavity that exert phagocytic and cytotoxic effects on sperm $^{198}$. Additionally, it is recognized that copper has direct spermicidal effects. Sperm incubated in the presence of copper show a reduction in motility, viability and fertilizing capacity ${ }^{199,200}$. Moreover, sperm transport is impaired by high copper content in the cervical mucus and tubal fluid of copper IUD users, which inhibits sperm penetration and tubal motility, respectively ${ }^{201-204}$. Indeed, one clinical study recovered significantly more sperm from the fallopian tubes of women using no contraception 15 to 30 minutes after insemination, while no sperm was found in the tubes of copper IUD users at the same post-coital time ${ }^{205}$.

\subsubsection{Injections}

Contraceptive injections are widely available, highly effective, long-acting reversible methods that became available in 1967. As of 2019, there are 74 million users of contraceptive injections worldwide, which accounts for $8 \%$ of global contraceptive use ${ }^{153}$. Since 1994, the prevalence of injectable methods has increased by $5 \%$ globally, driven by growth in Latin American, Southeastern Asian and sub-Saharan African countries ${ }^{153}$. Three types of injectable contraceptives have been developed: DMPA, norethindrone enanthate and various combination estrogen plus 
progestin formulations ${ }^{138}$. DMPA (Depo-Provera) is the most widely distributed worldwide. A single injection delivers a $150 \mathrm{mg}$ bolus of MPA intramuscularly, resulting in serum drug concentrations above the minimum concentration for ovulation inhibition $(0.2 \mathrm{ng} / \mathrm{mL}$ of MPA) over a period of three months. In fact, circulating blood levels of MPA exceed the threshold for contraceptive efficacy within 24 hours ${ }^{138}$. As with other hormonal contraceptives, substantial intraindividual and interindividual variability in pharmacokinetic profile is observed, due to differences in drug metabolism.

The contraceptive failure rate associated with DMPA injections is 0.22 per 100 participant-years, similar to that attributed to IUDs and implants ${ }^{162}$. The major mode of contraception action is ovulation inhibition. In randomized trial of DMPA users, authors found immediate depression of serum estradiol and progesterone levels within 24 hours post-injection ${ }^{206}$. Ovulation was suppressed for the entire 91-day treatment period ${ }^{206}$. In addition to anovulation, DMPA induces thickening of the cervical mucus and endometrial atrophy $207,208$. 


\subsection{Contraceptive Use and Ovarian Cancer}

\subsubsection{Evidence in the General-Risk Population}

This section describes the epidemiologic research on various contraceptive methods and ovarian cancer risk in the general population. Given the substantial body of literature on oral contraceptives and risk, emphasis will be placed on large pooled analyses and meta-analyses. Details on individual cohort studies investigating the relationship between oral contraceptive use and ovarian cancer risk are shown in Table 4. Available observational data on long-acting reversible contraceptives and ovarian cancer risk in the general population will also be presented.

\subsubsection{Combined Oral Contraceptives}

In 1999, the IARC reviewed the epidemiologic data on hormonal contraception and the risk of cancer at various sites. It was determined that oral contraceptive agents have a protective effect against cancers of the ovary ${ }^{209}$. To date, seven pooled analyses and one meta-analysis have quantified the role of oral contraceptives in ovarian cancer (Table 5). Of these, the largest collaborative pooled analysis included 45 epidemiologic studies (32 case-control, 13 prospective) across 21 countries based on 23,257 cases of ovarian cancer and 87,303 controls ${ }^{210}$. In agreement with the IARC, the authors observed a significant inverse relationship between a history of oral contraceptive use and ovarian cancer risk compared to never use (relative risk $[R R]=0.73 ; 95 \%$ CI 0.70-0.76). This protective effect was apparent within one to four years of use (RR=0.78; 95\% CI 0.73-0.83) and the RR decreased by $20 \%$ per five years of use (95\% CI $18-23 \%, \mathrm{p}<0 \cdot 0001)$. When time since last use was examined within categories of duration of use, there was a greater reduction in ovarian cancer risk observed with more recent use and an inverse trend with longer durations of use. Per five years of use, the RR declined by $29 \%$ for use ceased less than 10 years previously, $19 \%$ for use ceased 10-19 years previously and 15\% for use ceased 20-29 years previously ( $\mathrm{p}$-heterogeneity $=0.004)$. Further, risk reduction appeared to persist for at least 30 years after discontinuation of oral contraceptives $(\mathrm{RR}=0.83$; 95\% $\mathrm{CI} 0.73$ 0.95). In the analysis of age at first use, the authors also reported no significant heterogeneity across women who started oral contraceptives at different ages ( $p$-heterogeneity $=0.5$ ). A forest plot summarizing the findings of select case-control and cohort studies investigating the association between oral contraceptive use and ovarian cancer risk is shown in Figure 3. 
Similar findings emerged in an earlier pooled re-analysis of oral contraceptive use and ovarian cancer risk based on 2,768 cases and 6,273 controls from six studies conducted in three European countries ${ }^{211,212}$. In the most recent meta-analysis of 24 studies (17 case-control, seven prospective), 11 of which were published after the report by Beral et al., the multivariate RR was 0.73 (95\% CI 0.66-0.81) for ever use ${ }^{213}$. Additionally, authors observed a significant durationresponse relationship, with an ovarian cancer risk reduction of over $50 \%$ for women who used oral contraceptives for 10 or more years $(\mathrm{OR}=0.43 ; 95 \%$ CI $0.37-0.51)$. There was a trend toward greater reduction in ovarian cancer incidence with earlier age at first use and more recent use, but analyses were not stratified by duration of use. 


\section{Table 4. Summary of all cohort studies investigating the relationship between oral contraceptive use and ovarian cancer risk in the general population}

\begin{tabular}{|c|c|c|c|c|c|c|}
\hline $\begin{array}{l}\text { Author, } \\
\text { Year }\end{array}$ & Location & Source data & Cohort details & $\begin{array}{l}\text { Ever } \\
\text { use }\end{array}$ & $\begin{array}{l}\text { HR, RR }{ }^{\mathrm{a}} \\
(95 \% \mathrm{CI})\end{array}$ & Covariates \\
\hline $\begin{array}{l}\text { Hankinson, } \\
1995\end{array}$ & US & Nurses' Health Study & $\begin{array}{l}\text { Overall }(\mathrm{n}=121,700) \\
\text { Exposed }(592,056 \mathrm{p} \text {-years }) \\
\text { Unexposed }(599,301 \text { p-years })\end{array}$ & $\begin{array}{l}\text { Ever } \\
\text { use }\end{array}$ & $1.08(0.83-1.43)$ & $\begin{array}{l}\text { Age, parity, menopausal status, age } \\
\text { at menarche, smoking, tubal } \\
\text { ligation, Quetelet's Index }\end{array}$ \\
\hline $\begin{array}{l}\text { Vessey and } \\
\text { Painter, } 1995\end{array}$ & $\begin{array}{l}\text { England, } \\
\text { Scotland }\end{array}$ & $\begin{array}{l}\text { Oxford } \quad \text { Family } \\
\text { Planning Association } \\
\text { contraceptive study }\end{array}$ & $\begin{array}{l}\text { Overall }(\mathrm{n}=17,032) \\
\text { Exposed }(\mathrm{n}=3,520 \text { women } \geq 8 \\
\text { years use }) \\
\text { Unexposed }(\mathrm{n}=5,881)\end{array}$ & $\begin{array}{l}\text { Ever } \\
\text { use }\end{array}$ & $0.3(0.1-0.7)$ & Age, parity \\
\hline Kumle, 2004 & $\begin{array}{l}\text { Norway, } \\
\text { Sweden }\end{array}$ & $\begin{array}{l}\text { Norwegian-Swedish } \\
\text { Women's Lifestyle } \\
\text { and Health Cohort } \\
\text { Study }\end{array}$ & $\begin{array}{l}\text { Overall }(\mathrm{n}=103,551) \\
\text { Exposed }(\mathrm{n}=75,533) \\
\text { Unexposed }(\mathrm{n}=28,019)\end{array}$ & $\begin{array}{l}\text { Ever } \\
\text { use }\end{array}$ & $0.6(0.5-0.8)$ & $\begin{array}{l}\text { Age, parity, menopausal status, } \\
\text { HRT, country }\end{array}$ \\
\hline $\begin{array}{l}\text { Vessey and } \\
\text { Painter, } 2006\end{array}$ & UK & $\begin{array}{l}\text { Oxford } \quad \text { Family } \\
\text { Planning Association } \\
\text { contraceptive study }\end{array}$ & $\begin{array}{l}\text { Overall }(\mathrm{n}=17,032) \\
\text { Exposed }(187,000 \text { p-years }) \\
\text { Unexposed }(301,000 \text { p-years })\end{array}$ & $\begin{array}{l}\text { Ever } \\
\text { use }\end{array}$ & $0.5(0.4-0.7)$ & $\begin{array}{l}\text { Age, parity, BMI, smoking, social } \\
\text { class, height, age at first term } \\
\text { pregnancy, age at first marriage }\end{array}$ \\
\hline $\begin{array}{l}\text { Hannaford, } \\
2007\end{array}$ & UK & $\begin{array}{l}\text { Royal College of } \\
\text { General Practitioners' } \\
\text { Oral Contraception } \\
\text { Study }\end{array}$ & $\begin{array}{l}\text { Overall }(\mathrm{n}=45,950) \\
\text { Exposed }(\mathrm{n}=28,762) \\
\text { Unexposed: }(\mathrm{n}=17,188)\end{array}$ & $\begin{array}{l}\text { Ever } \\
\text { use }\end{array}$ & $0.51(0.33-0.78)$ & Age, parity, smoking, social status \\
\hline $\begin{array}{l}\text { Tworoger, } \\
2007\end{array}$ & US & Nurses' Health Study & $\begin{array}{l}\text { Overall }(\mathrm{n}=107,900) \\
\text { Exposed }(99,479 \text { p-years }) \\
\text { Unexposed } \quad(1,403,441 \quad \mathrm{p} \text { - } \\
\text { years })\end{array}$ & $\begin{array}{l}>10 \\
\text { years of use }\end{array}$ & $0.62(0.37-1.04)$ & $\begin{array}{l}\text { Age, BMI, parity, history of tubal } \\
\text { ligation, smoking history, age at } \\
\text { menarche, age at menopause, } \\
\text { duration of postmenopausal } \\
\text { hormone use }\end{array}$ \\
\hline $\begin{array}{l}\text { Dorjgochoo, } \\
2009\end{array}$ & China & $\begin{array}{l}\text { Shanghai Women's } \\
\text { Health Study }\end{array}$ & $\begin{array}{l}\text { Overall }(\mathrm{n}=66,661) \\
\text { Exposed }(\mathrm{n}=12,957) \\
\text { Unexposed }(\mathrm{n}=15,557)\end{array}$ & $\begin{array}{l}\text { Ever } \\
\text { use }\end{array}$ & $1.1(0.66-1.84)$ & $\begin{array}{l}\text { Age, parity, menopausal status, } \\
\text { BMI, family history, age at } \\
\text { menarche, smoking, breastfeeding } \\
\text {, education, physical activity, } \\
\text { other contraceptive methods }\end{array}$ \\
\hline $\begin{array}{l}\text { Rosenblatt, } \\
2009\end{array}$ & China & $\mathrm{n} / \mathrm{a}$ & $\begin{array}{l}\text { Overall }(\mathrm{n}=258,956) \\
\text { Exposed }(\mathrm{n}=37,319 ; 349,539 \\
\text { p-years }) \\
\text { Unexposed } \quad(2,008,845 \quad \mathrm{p}- \\
\text { years })\end{array}$ & $\begin{array}{l}\text { Ever } \\
\text { use }\end{array}$ & $1.17(0.86-1.60)$ & $\begin{array}{l}\text { Age, parity, injectable } \\
\text { contraceptive use }\end{array}$ \\
\hline Braem, 2010 & Netherlands & $\begin{array}{l}\text { Netherlands Cohort } \\
\text { Study on Diet and } \\
\text { Cancer }\end{array}$ & $\begin{array}{l}\text { Overall }(\mathrm{n}=62,573) \\
\text { Exposed }(8,669 \text { p-years }) \\
\text { Unexposed }(25,917 \text { p-years })\end{array}$ & $\begin{array}{l}\text { Ever } \\
\text { use }\end{array}$ & $0.71(0.52-0.97)$ & Age, parity \\
\hline
\end{tabular}




\begin{tabular}{|c|c|c|c|c|c|c|}
\hline Tsilidis, 2011 & Europe & $\begin{array}{l}\text { European Prospective } \\
\text { Investigation into } \\
\text { Cancer and Nutrition }\end{array}$ & $\begin{array}{l}\text { Overall }(n=327,396) \\
\text { Exposed }(n=192,836) \\
\text { Unexposed }(n=132,923)\end{array}$ & $\begin{array}{l}\text { Ever } \\
\text { use }\end{array}$ & $0.86(0.73-1.00)$ & $\begin{array}{l}\text { Age, parity, menopausal status, } \\
\text { BMI, smoking, center, unilateral } \\
\text { oophorectomy, hysterectomy, } \\
\text { menopausal hormones, age at } \\
\text { menarche }\end{array}$ \\
\hline Yang, 2012 & US & $\begin{array}{l}\text { NIH-AARP Diet and } \\
\text { Health Study }\end{array}$ & $\begin{array}{l}\text { Overall }(n=169,391) \\
\text { Exposed }(n=67,870) \\
\text { Unexposed }(n=100,304)\end{array}$ & $\begin{array}{l}\text { Ever } \\
\text { use }\end{array}$ & $0.74(0.63-0.87)$ & $\begin{array}{l}\text { Age, parity, menopausal hormone } \\
\text { therapy }\end{array}$ \\
\hline $\begin{array}{l}\text { Vessey and } \\
\text { Yeates, } 2013\end{array}$ & UK & $\begin{array}{l}\text { Oxford Family } \\
\text { Planning Association } \\
\text { contraceptive study } \\
\end{array}$ & Overall $(n=17,032)$ & $\begin{array}{l}\text { Ever } \\
\text { use }\end{array}$ & $0.5(0.4-0.7)$ & $\begin{array}{l}\text { Age, parity, social class, smoking, } \\
\text { BMI, age at first marriage }\end{array}$ \\
\hline Fortner, 2015 & Europe & $\begin{array}{l}\text { European Prospective } \\
\text { Investigation into } \\
\text { Cancer and Nutrition }\end{array}$ & $\begin{array}{l}\text { Overall }(n=334,126) \\
\text { Exposed }(n=192,207) \\
\text { Unexposed }(n=133,008) \\
\end{array}$ & $\begin{array}{l}\text { Ever } \\
\text { use }\end{array}$ & $0.84(0.73-0.96)$ & $\begin{array}{l}\text { Ever full-term pregnancy, } \\
\text { menopausal status at recruitment, } \\
\text { age at menopause, ever HRT use }\end{array}$ \\
\hline Huang, 2015 & China & $\begin{array}{l}\text { Shanghai Women's } \\
\text { Health Study }\end{array}$ & $\begin{array}{l}\text { Overall }(n=70,259) \\
\text { Exposed }(n=54,113) \\
\text { Unexposed }(n=16,146)\end{array}$ & $\begin{array}{l}\text { Ever } \\
\text { use }\end{array}$ & $0.86(0.60-1.24)$ & $\begin{array}{l}\text { Age, education, years of ovulation, } \\
\text { irregular ovulatory cycles, first- } \\
\text { degree family history of cancer, } \\
\text { regular exercise in past } 5 \text { years, } \\
\text { other contraceptive methods }\end{array}$ \\
\hline Shafrir, 2017 & US & $\begin{array}{l}\text { Nurses' Health Study } \\
\text { II }\end{array}$ & $\begin{array}{l}\text { Overall }(n=110,929) \\
\text { Total exposed } \\
(n=129,923) \\
\text { Total unexposed } \\
(n=18,994)\end{array}$ & $\begin{array}{l}\geq 15 \\
\text { years of } \\
\text { use }\end{array}$ & $0.43(0.18-1.04)$ & $\begin{array}{l}\text { Age, calendar time, tubal ligation, } \\
\text { parity, breastfeeding duration, and } \\
\text { missing breastfeeding duration, } \\
\text { endometriosis, BMI }\end{array}$ \\
\hline Iversen, 2017 & UK & $\begin{array}{l}\text { Royal College of } \\
\text { General Practitioners' } \\
\text { Oral Contraception } \\
\text { Study }\end{array}$ & $\begin{array}{l}\text { Overall }(n=46,022) \\
\text { Exposed }(n=22,920) \\
\text { Unexposed }(n=23,102)\end{array}$ & $\begin{array}{l}\text { Ever } \\
\text { use }\end{array}$ & $0.66(0.58-0.76)$ & $\begin{array}{l}\text { Age, parity, smoking, and social } \\
\text { class }\end{array}$ \\
\hline Bethea, 2017 & US & $\begin{array}{l}\text { Black Women's } \\
\text { Health Study }\end{array}$ & $\begin{array}{l}\text { Overall }(\mathrm{n}=53,141) \\
\text { Exposed }(343,045 \mathrm{p}- \\
\text { years }) \\
\text { Unexposed }(147,799 \mathrm{p}- \\
\text { years })\end{array}$ & $\begin{array}{l}\geq 10 \\
\text { years of } \\
\text { use }\end{array}$ & $0.50(0.30-0.83)$ & $\begin{array}{l}\text { Age, questionnaire cycle, parity, } \\
\text { lactation, age at first birth, age at } \\
\text { last birth, hysterectomy, tubal } \\
\text { ligation, menopausal status, use of } \\
\text { postmenopausal female hormones, } \\
\text { educational attainment, BMI }\end{array}$ \\
\hline Michels, 2018 & US & $\begin{array}{l}\text { NIH-AARP Diet and } \\
\text { Health Study }\end{array}$ & $\begin{array}{l}\text { Overall }(n=196,536) \\
\text { Exposed }(n=78,393) \\
\text { Unexposed }(n=118,144)\end{array}$ & $\begin{array}{l}\text { Ever } \\
\text { use }\end{array}$ & $0.74(0.65-0.84)$ & $\begin{array}{l}\text { Age, race, BMI, baseline smoking } \\
\text { status, age at menarche }\end{array}$ \\
\hline Iversen, 2018 & Denmark & $\begin{array}{l}\text { Danish Sex Hormone } \\
\text { Register Study }\end{array}$ & $\begin{array}{l}\text { Overall }(\mathrm{n}=1,879,227) \\
\text { Exposed }(7,751,904 \mathrm{p}- \\
\text { years of current or recent }\end{array}$ & $\begin{array}{l}\text { Ever } \\
\text { use }\end{array}$ & $0.53(0.45-0.64)$ & $\begin{array}{l}\text { Calendar year, parity, age, } \\
\text { education, tubal sterilization, } \\
\text { hysterectomy, endometriosis, }\end{array}$ \\
\hline
\end{tabular}




\begin{tabular}{|l|l|l|l|l|l|}
\hline & & $\begin{array}{l}\text { use) } \\
\text { Unexposed }(8,150,250 \\
\text { p-years }\end{array}$ & $\begin{array}{l}\text { polycystic ovary syndrome, family } \\
\text { history of breast or ovarian cancer }\end{array}$ \\
\hline
\end{tabular}

Body mass index (BMI); hormone replacement therapy (HRT)

${ }^{a}$ Relative risk (RR) or hazard ratio (HR) for ever vs never oral contraceptive use. 
Table 5. Summary of all pooled analyses and meta-analyses investigating the relationship between oral contraceptive use and ovarian cancer risk in the general population

\begin{tabular}{|c|c|c|c|c|c|c|}
\hline $\begin{array}{l}\text { Author, } \\
\text { Year }\end{array}$ & Country & $\begin{array}{l}\text { Type of } \\
\text { study }\end{array}$ & Study details & Outcome & OR $(95 \% \mathrm{CI})^{\mathrm{a}}$ & Covariates \\
\hline $\begin{array}{l}\text { Franceschi, } \\
1991\end{array}$ & Europe & $\begin{array}{l}\text { Pooled } \\
\text { analysis }\end{array}$ & $\begin{array}{l}3 \text { case-control studies } \\
\text { Cases }(n=971) \\
\text { Hospital controls }(\mathrm{n}=2,258)\end{array}$ & $\begin{array}{l}\text { Epithelial ovarian } \\
\text { cancer }\end{array}$ & $0.6(0.4-0.8)$ & $\begin{array}{l}\text { Study, age, marital status, } \\
\text { socioeconomic status, parity, } \\
\text { menopause, contraceptive habits }\end{array}$ \\
\hline $\begin{array}{l}\text { Whittemore, } \\
1992\end{array}$ & US & $\begin{array}{l}\text { Pooled } \\
\text { analysis }\end{array}$ & $\begin{array}{l}12 \text { case-control studies } \\
\text { Cases }(\mathrm{n}=12,197) \\
\text { Controls }(8,893)\end{array}$ & $\begin{array}{l}\text { Epithelial ovarian } \\
\text { cancer }\end{array}$ & $\begin{array}{l}0.70(0.52-0.94) \\
\text { (hospital-based) } \\
0.66(0.55-0.78) \\
\text { (population-based) }\end{array}$ & Study, age, parity \\
\hline Harris, 1992 & Europe & $\begin{array}{l}\text { Pooled } \\
\text { analysis }\end{array}$ & $\begin{array}{l}12 \text { case-control studies } \\
\text { Cases }(\mathrm{n}=327) \\
\text { Controls }(\mathrm{n}=4,144)\end{array}$ & $\begin{array}{l}\text { Borderline ovarian } \\
\text { cancer }\end{array}$ & $1.1(0.7-1.9)$ & Study, age, parity \\
\hline \multirow[t]{2}{*}{$\begin{array}{l}\text { Horn-Ross, } \\
1992\end{array}$} & \multirow[t]{2}{*}{ US } & \multirow[t]{2}{*}{$\begin{array}{l}\text { Pooled } \\
\text { analysis }\end{array}$} & $\begin{array}{l}4 \text { case-control studies } \\
\text { Cases }(n=38) \\
\text { Controls }(n=1,142)\end{array}$ & Germ cell tumours & $2.0(0.77-5.1)$ & \multirow[t]{2}{*}{ Study, age, year of birth } \\
\hline & & & $\begin{array}{l}\text { Cases }(n=45) \\
\text { Controls }(n=2,617)\end{array}$ & $\begin{array}{l}\text { Sex cord-stromal } \\
\text { tumours }\end{array}$ & $0.37(0.16-0.83)$ & \\
\hline $\begin{array}{l}\text { Bosetti, } \\
2002\end{array}$ & Europe & $\begin{array}{l}\text { Pooled } \\
\text { analysis }\end{array}$ & $\begin{array}{l}6 \text { case-control studies } \\
\text { Cases }(\mathrm{n}=2,768) \\
\text { Hospital controls }(\mathrm{n}=6,274)\end{array}$ & $\begin{array}{l}\text { Epithelial ovarian } \\
\text { cancer }\end{array}$ & $0.66(0.56-0.79)$ & $\begin{array}{l}\text { Study, age, year, socioeconomic } \\
\text { status, parity, menopause, age at } \\
\text { menopause }\end{array}$ \\
\hline Beral, 2008 & International & $\begin{array}{l}\text { Pooled } \\
\text { analysis }\end{array}$ & $\begin{array}{l}13 \text { prospective } \\
32 \text { case-control } \\
\text { Cases }(n=23,257) \\
\text { Controls }(n=87,303)\end{array}$ & $\begin{array}{l}\text { Epithelial or non- } \\
\text { epithelial ovarian } \\
\text { cancer }\end{array}$ & $0.73(0.70-0.76)$ & Study, age, parity, hysterectomy \\
\hline $\begin{array}{l}\text { Havrilesky, } \\
2013\end{array}$ & International & $\begin{array}{l}\text { Meta- } \\
\text { analysis }\end{array}$ & $\begin{array}{l}17 \text { case-control } \\
7 \text { prospective }\end{array}$ & Any ovarian cancer & $0.73(0.66-0.81)$ & $\mathrm{n} / \mathrm{a}$ \\
\hline $\begin{array}{l}\text { Wentzensen, } \\
2016\end{array}$ & International & $\begin{array}{l}\text { Pooled } \\
\text { analysis }\end{array}$ & $\begin{array}{l}21 \text { studies } \\
\text { Cases }(\mathrm{n}=5,584) \\
\text { Controls }(\mathrm{n} \cong 1.3 \text { million })\end{array}$ & $\begin{array}{l}\text { Invasive epithelial } \\
\text { ovarian cancer }\end{array}$ & $0.84(0.79-0.89)$ & Age, parity \\
\hline
\end{tabular}

\footnotetext{
${ }^{a}$ Odds ratio for ever vs. never oral contraceptive use
} 


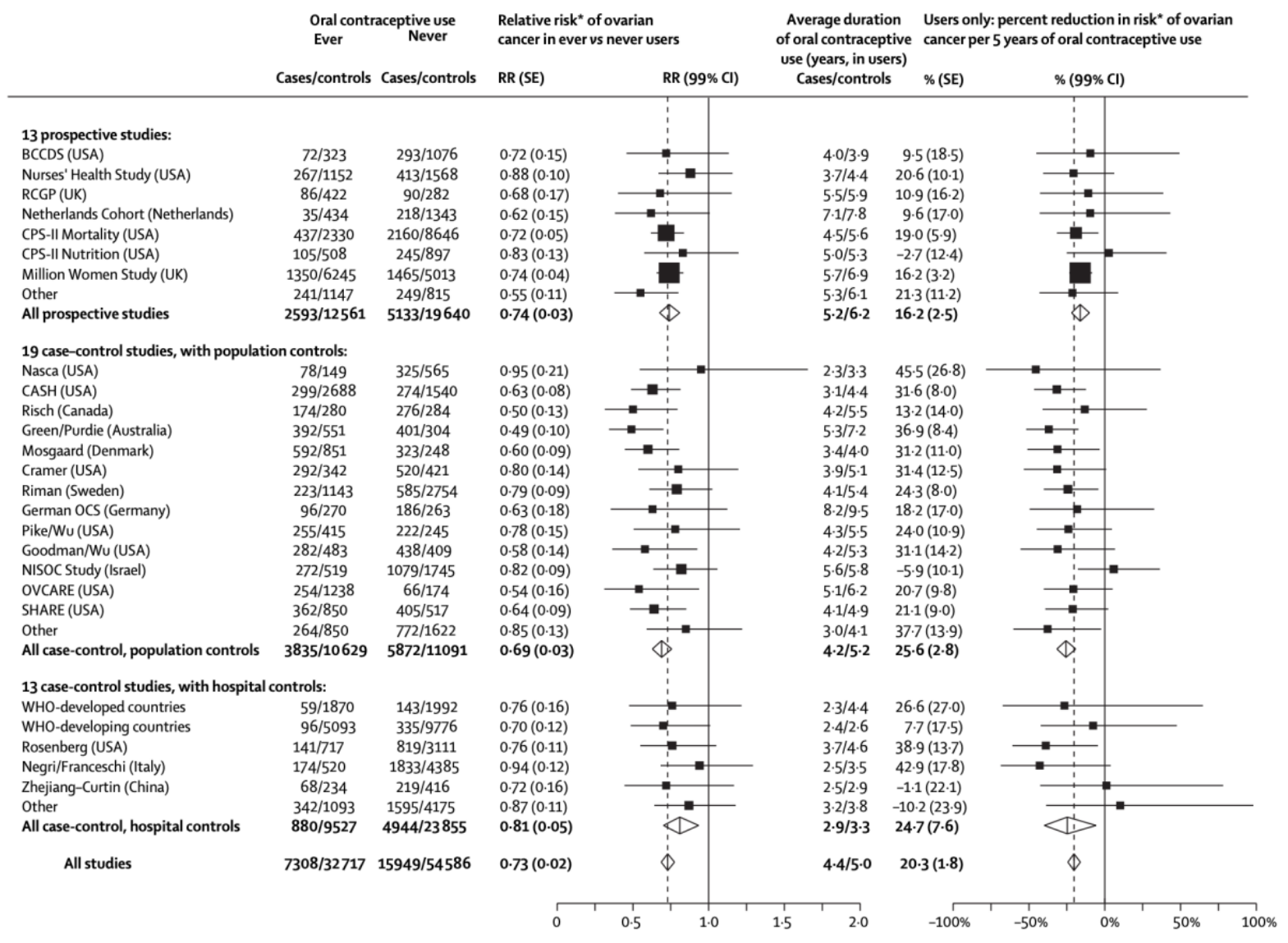

Figure 3. Forest plot summarizing the findings of select case-control and cohort studies investigating oral contraceptive use and ovarian cancer risk in the general population

Reproduced from Beral V, Doll R, Hermon C, Peto R, Reeves G, Schouten LJ, van den Brandt PA. Ovarian cancer and oral contraceptives: collaborative reanalysis of data from 45 epidemiological studies including 23,257 women with ovarian cancer and 87,303 controls. Lancet. 2008;371(9609):303-14 by permission from Elsevier.

Over the past five decades, the estrogen and progestin content of oral contraceptive pills have decreased rapidly in effort to reduce undesirable side-effects without compromising contraceptive efficacy ${ }^{\mathbf{1 5 2 , 2 1 4}}$. Low-dose formulations are effective at suppressing ovulation, but it is unclear how changes in hormonal dose and potency affect ovarian cancer risk, particularly for those at high risk ${ }^{\mathbf{2 1 5 , 2 1 6}}$. Available data in the general population has been mixed. A metaanalysis considered six case-control studies examining the effect of estrogen and progestin formulation on ovarian cancer risk. Authors concluded that there was no relationship between the estrogen or progestin dose of oral contraceptive pills and risk of ovarian cancer in the general population $^{213}$. In contrast, more recent data from the Nurses' Health Study II suggest that women who used high-dose estrogen formulations had a non-significant increased risk of ovarian cancer 
compared to women who used low-dose estrogen formulations $(\mathrm{HR}=1.34 ; 95 \% \mathrm{CI}$ 0.91-1.95 for high). This finding may have been driven by the positive association with short-term use of mestranol $(\mathrm{HR}=1.83 ; 95 \% \mathrm{CI} 1.16-2.88)$ or first generation progestins $(\mathrm{HR}=1.72 ; 95 \% \mathrm{CI}$ 1.11-2.65) compared to never users ${ }^{217}$. There was no association between hormone potency and risk. Other studies have found an inverse relationship between progestin potency and risk, such that older, high-progestin formulations confer greater protection than low-progestin formulations $213,218-223$

\subsubsection{Contraceptive Injections}

The growing uptake of LARC methods has prompted further research into their noncontraceptive benefits, particularly in terms of ovarian cancer prevention. Ovulation inhibition via suppression of pituitary gonadotropins is the chief biological mechanism by which oral contraceptives, implants and injections may impart protection against the development of ovarian cancer (see Section 1.2.3). Indeed, a recent pooled analysis of seven case-control studies in the Ovarian Cancer Association Consortium concluded a reduced risk of ovarian cancer with use of DMPA shots $(\mathrm{OR}=0.65 ; 95 \%$ CI $0.50-0.85)$, which is in line with the majority of other retrospective data ${ }^{224-226}$. Cohort studies that have explored injection use and ovarian cancer risk have showed inconsistent results. In the Shanghai Women's Health Study, injectables were not associated with risk $(\mathrm{HR}=1.33$; $95 \%$ CI 0.58-3.04), whereas the Danish Sex Register Hormone Study observed increased risk with use of DMPA shots compared to never use of any hormonal contraceptive $(\mathrm{RR}=6.56 \text {; 95\% CI 2.11-20.40 })^{227,228}$. Both studies were limited by a low prevalence of injection users and imprecise risk estimates.

\subsubsection{Implants}

With respect to subdermal contraceptive implants, only one study estimated risk of ovarian cancer among implant users in the general population ${ }^{227}$. The authors reported a nonsignificant inverse association between implant use and ovarian cancer risk compared to never users $(\mathrm{RR}=0.51$; 95\% CI 0.07-3.64); however, any protective effect may have been underestimated because history of contraceptive use prior to study entry was not collected. 


\subsubsection{Intrauterine Devices}

A greater body of evidence has explored the associations between IUD use and ovarian cancer risk (Table 6). Of note, two recent meta-analyses both demonstrated that use of an IUD decreases risk of ovarian cancer, reporting ORs of 0.68 (95\% CI 0.62-0.75) and 0.67 (95\% CI 0.60-

$0.74)^{229,230}$. Population-based cohort studies have specifically linked this protective effect to use of the levonorgestrel-bearing, hormonal IUD ${ }^{231,232}$. The data with respect to nonhormonal IUDs remain controversial. A pooled analysis of the New England Case-Control Study and Nurses' Health Studies reported no association between IUD use and ovarian cancer risk, wherein the majority of IUD use was attributed to nonhormonal methods $(\mathrm{RR}=0.94 ; 95 \% \mathrm{CI} 0.81-1.08)^{233}$. Conversely, findings from the Shanghai Women's Health Study indicated that long-term IUD use greater than 20 years was associated with a $38 \%$ reduced ovarian cancer risk in China, where the high prevalence of IUD use was dominated by stainless steel methods (HR=0.62; 95\% CI $0.40-0.97)^{228}$. Interestingly, Tworoger and colleagues observed elevated ovarian cancer risk with nonhormonal IUD use in an earlier analysis of Nurses' Health Study, which was attributed to IUD-related peritoneal inflammation $(\mathrm{RR}=1.76 \text {; 95\% CI 1.08-2.85 })^{234}$. 
Table 6. Summary of cohort studies, pooled analyses and meta-analyses investigating the relationship between use of an intrauterine device and ovarian cancer risk in the general population

\begin{tabular}{|c|c|c|c|c|c|c|c|c|}
\hline $\begin{array}{l}\text { Author, } \\
\text { Year }\end{array}$ & Location & $\begin{array}{l}\text { Type of } \\
\text { study }\end{array}$ & Study details & Exposure & Comparison & $\begin{array}{l}\text { Subgroup } \\
\text { analysis }\end{array}$ & $\begin{array}{l}\text { OR, HR, RR } \\
(95 \% \text { CI })\end{array}$ & Covariates \\
\hline $\begin{array}{l}\text { Franceschi } \\
1991\end{array}$ & Europe & $\begin{array}{l}\text { Pooled } \\
\text { analysis }\end{array}$ & $\begin{array}{l}3 \text { case-control studies } \\
\text { Cases }(n=971) \\
\text { Hospital controls } \\
(n=2,258) \\
\text { Exposed }(n=116)\end{array}$ & Any IUD & Ever use & All & $0.8(0.5-1.3)$ & $\begin{array}{l}\text { Age, socio-cultural } \\
\text { indicators, marital } \\
\text { status, menopausal } \\
\text { status, parity, } \\
\text { contraceptive habits, } \\
\text { study centre }\end{array}$ \\
\hline $\begin{array}{l}\text { Kotsopoulos, } \\
2013\end{array}$ & USA & $\begin{array}{l}\text { Pooled } \\
\text { analysis }\end{array}$ & $\begin{array}{l}\text { NECC, NHSI, NHSII } \\
\text { Cases }(n=1,771)\end{array}$ & Any IUD & Ever use & $\begin{array}{l}\text { Dominant } \\
\text { Nondominant }\end{array}$ & $\begin{array}{l}0.83(0.65-1.05) \\
1.70(0.68-4.26)\end{array}$ & $\begin{array}{l}\text { Age, oral contraceptive } \\
\text { use, parity, tubal } \\
\text { ligation, family history } \\
\text { of breast or ovarian } \\
\text { cancer, study phase, } \\
\text { center for NECC; time } \\
\text { period, cohort, } \\
\text { histology for NHS }\end{array}$ \\
\hline $\begin{array}{l}\text { Wheeler, } \\
2019\end{array}$ & International & $\begin{array}{l}\text { Meta- } \\
\text { analysis }\end{array}$ & $\begin{array}{l}9 \text { case-control studies } \\
2 \text { cohort studies } \\
\text { Cases }(n=5,133) \\
\text { Controls }(n=183,035)\end{array}$ & Any IUD & Ever use & All & $0.68(0.62-0.75)$ & $\mathrm{n} / \mathrm{a}$ \\
\hline $\begin{array}{l}\text { Balayla, } \\
2020\end{array}$ & International & $\begin{array}{l}\text { Meta- } \\
\text { analysis }\end{array}$ & $\begin{array}{l}5 \text { case-control studies } \\
2 \text { cohort studies }\end{array}$ & $\begin{array}{l}\text { Any IUD } \\
\text { LNG IUD }\end{array}$ & Ever use & All & $\begin{array}{l}0.67(0.60-0.74) \\
0.58(0.47-0.71)\end{array}$ & $\mathrm{n} / \mathrm{a}$ \\
\hline Yang, 2021 & USA & $\begin{array}{l}\text { Pooled } \\
\text { analysis }\end{array}$ & NECC, NHSI, NHSII & Any IUD & Ever use & All & $0.96(0.81-1.14)$ & $\begin{array}{l}\text { Age, body mass index, } \\
\text { age at menarche, parity, } \\
\text { oral contraceptive use, } \\
\text { history of tubal ligation, } \\
\text { family history of } \\
\text { ovarian cancer, study } \\
\text { center, study phase } \\
\text { (NECC) }\end{array}$ \\
\hline $\begin{array}{l}\text { Tworoger, } \\
2007\end{array}$ & USA & Cohort & $\begin{array}{l}\text { Overall }(n=107,900) \\
\text { Cases }(n=625) \\
\text { Exposed }(64,847 \mathrm{p}- \\
\text { years })\end{array}$ & Any IUD & Ever use & All & $1.76(1.08-2.85)$ & $\begin{array}{l}\text { Age, age at menarche, } \\
\text { age at menopause, } \\
\text { BMI, oral contraceptive } \\
\text { use, parity, } \\
\text { postmenopausal }\end{array}$ \\
\hline
\end{tabular}




\begin{tabular}{|c|c|c|c|c|c|c|c|c|}
\hline & & & & & & & & $\begin{array}{l}\text { hormone use, tubal } \\
\text { ligation, smoking }\end{array}$ \\
\hline Huang, 2015 & China & Cohort & $\begin{array}{l}\text { Overall }(\mathrm{n}=70,529) \\
\text { Cases }(\mathrm{n}=174) \\
\text { Exposed }(\mathrm{n}=39,054)\end{array}$ & Any IUD & Ever use & All & $0.79(0.55-1.13)$ & $\begin{array}{l}\text { Age, education, years } \\
\text { of ovulation, irregular } \\
\text { ovulatory cycles, first- } \\
\text { degree family history of } \\
\text { cancer, BMI, physical } \\
\text { activity, other } \\
\text { contraceptive methods }\end{array}$ \\
\hline Soini, 2016 & Finland & Cohort & $\begin{array}{l}\text { Overall }(n=93,843) \\
\text { Cases }(n=77)\end{array}$ & LNG IUD & Ever use & All & $0.59(0.47-0.73)$ & $\mathrm{n} / \mathrm{a}$ \\
\hline Jareid, 2018 & Norway & Cohort & $\begin{array}{l}\text { Overall }(\mathrm{n}=104,318) \\
\text { Exposed }(\mathrm{n}=9,146)\end{array}$ & LNG IUD & Ever use & All & $0.53(0.32-0.88)$ & $\begin{array}{l}\text { Age, parity, BMI, oral } \\
\text { contraceptive use, } \\
\text { menopausal status, } \\
\text { maternal history of } \\
\text { breast cancer, physical } \\
\text { activity }\end{array}$ \\
\hline $\begin{array}{l}\text { Iversen, } \\
2018\end{array}$ & Denmark & Cohort & $\begin{array}{l}\text { Overall }(\mathrm{n}=104,318 ; \\
21.4 \text { million p-years }) \\
\text { Exposed }(172,265 \mathrm{p}- \\
\text { years })\end{array}$ & LNG IUD & Ever use & All & $0.84(0.53-1.35)$ & $\begin{array}{l}\text { Calendar year, parity, } \\
\text { age, education, tubal } \\
\text { sterilisation, } \\
\text { hysterectomy, } \\
\text { endometriosis, } \\
\text { polycystic ovary } \\
\text { syndrome, family } \\
\text { history of breast or } \\
\text { ovarian cancer }\end{array}$ \\
\hline
\end{tabular}

Odds ratio (OR); hazard ratio (HR); relative risk (RR); New England Case Control Study (NECC); Nurses' Health Study (NHS); Oral contraceptive (OC); Body mass index (BMI); levonorgestrel-releasing IUD (LNG IUD) 


\subsubsection{Evidence in High-Risk Populations}

This section reviews the literature regarding oral contraceptive use and ovarian cancer risk conducted among BRCA mutation carriers with a focus on robust meta-analyses and large cohort studies. The role of LARC methods, including intrauterine devices, implants and injections, has not been evaluated in the context of $B R C A$-associated ovarian cancer.

Since 1998, three meta-analyses and 12 epidemiologic studies have evaluated the impact of oral contraceptive use on ovarian cancer risk in high-risk populations, supporting a strong protective effect. The key features of these studies are summarized in Table 7 and reviewed in References 116, 126 and 237. A meta-analysis including three case-control studies published through 2009 showed that women who carried a BRCAl mutation had a $44 \%$ lower risk of ovarian cancer with oral contraceptive use compared to those who never users $\left(\mathrm{OR}=0.56\right.$; 95\% CI 0.49-0.69) ${ }^{235}$. For women who carried a $B R C A 2$ mutation, oral contraceptive use conferred a $51 \%$ risk reduction compared to never use $(\mathrm{OR}=0.49 ; 95 \%$ CI 0.32-0.77). Similar findings were reported in a larger meta-analysis based on data from four case-control studies and one retrospective cohort study published through $2010^{236}$. Results indicated significantly reduced risks of ovarian cancer for $B R C A$ mutation carriers who used oral contraceptives (summary relative risk $[\mathrm{SRR}]=0.51,95 \%$ CI 0.40-0.65 for BRCA1; SRR=0.52, 95\% CI 0.31-0.87 for BRCA2). Further, a weak protective effect was observed with a duration of use of at least one year (SRR $=0.96$; 95\% CI 0.94-0.97) and risk reduction increased linearly per year of use (p-trend <0.01). Moorman et al. conducted the most recent systematic review and meta-analysis of oral contraceptives and ovarian cancer risk from six studies in high-risk groups published through $2013^{237}$. Authors reported on 1,763 cases (1,353 BRCA1, 277 BRCA2) and 2,592 controls (2,160 BRCA1, 423 BRCA2). A significantly decreased risk of ovarian cancer was observed among $B R C A$ mutation carrier ever users compared to never users $(\mathrm{OR}=0.58 ; 95 \% \mathrm{CI} 0.46-0.73)$. This was primarily driven by the protective effect of oral contraceptives in $B R C A 1$ mutation carriers separately $(\mathrm{OR}=0.55$; $95 \% \mathrm{CI}$ 0.47-0.66). For women with a BRCA2 mutation, the inverse relationship did not reach statistical significance due to the smaller sample size (OR=0.65; 95\% CI 0.34-1.24). Analysis of duration of use and its association with risk was not performed. 
Table 7. Summary of epidemiologic studies investigating the relationship between oral contraceptive use and ovarian cancer risk in $B R C A$ mutation carriers

\begin{tabular}{|c|c|c|c|c|c|c|c|}
\hline Author, Year & Location & Type of study & Study details & Comparison & $\begin{array}{l}\text { Subgroup } \\
\text { analysis }\end{array}$ & $\begin{array}{l}\text { OR, HR } \mathbf{H R}^{\mathrm{a}} \\
(95 \% \mathrm{CI})\end{array}$ & Covariates \\
\hline Narod, 1998 & International & Case-control & $\begin{array}{l}\text { Cases }(\mathrm{n}=207 ; 179 \\
B R C A 1,28 B R C A 2) \\
\text { Sister-controls }(\mathrm{n}=161 ; \\
50 B R C A 1,3 B R C A 2)\end{array}$ & Ever use & $\begin{array}{l}\text { BRCA1/2 } \\
\text { BRCA1 } \\
\text { BRCA2 }\end{array}$ & $\begin{array}{l}0.4(0.2-0.7) \\
0.5(0.3-0.9) \\
0.4(0.2-1.1)\end{array}$ & $\begin{array}{l}\text { Year of birth, parity, } \\
\text { age at the delivery of a first } \\
\text { child, and geographic area } \\
\text { of residence }\end{array}$ \\
\hline Modan, 2001 & Israel & $\begin{array}{l}\text { Matched case- } \\
\text { only }\end{array}$ & $\begin{array}{l}\text { Carrier cases }(\mathrm{n}=240) \\
\text { Population controls } \\
(\mathrm{n}=2,257)\end{array}$ & $\begin{array}{l}0.1-1.9 \text { years } \\
2.0-4.9 \text { years } \\
\geq 5 \text { years }\end{array}$ & BRCA1/2 & $\begin{array}{l}1.14(0.67-1.94) \\
0.77(0.41-1.44) \\
1.07(0.63-1.83)\end{array}$ & $\begin{array}{l}\text { Age, family history of } \\
\text { breast or ovarian cancer, } \\
\text { personal history of breast } \\
\text { cancer, history of } \\
\text { gynecologic surgery, ethnic } \\
\text { background }\end{array}$ \\
\hline Narod, 2001 & International & Case-control & $\begin{array}{l}\text { Cases }(n=232) \\
\text { Controls }(n=232)\end{array}$ & Ever use & $\begin{array}{l}\text { BRCA1/2 } \\
\text { BRCA1 } \\
\text { BRCA2 } \\
\end{array}$ & $\begin{array}{l}0.44(0.28-0.68) \\
0.48(0.29-0.80) \\
0.35(0.15-0.83) \\
\end{array}$ & $\mathrm{n} / \mathrm{a}$ \\
\hline $\begin{array}{l}\text { McGuire, } \\
2004\end{array}$ & US & $\begin{array}{l}\text { Matched case- } \\
\text { control }\end{array}$ & $\begin{array}{l}\text { Carrier cases }(n=46) \\
\text { Untested population } \\
\text { controls }(n=568)\end{array}$ & Ever use & BRCAI & $0.54(0.26-1.13)$ & $\begin{array}{l}\text { Age, number of full-term } \\
\text { pregnancies, race/ethnicity }\end{array}$ \\
\hline $\begin{array}{l}\text { Whittemore, } \\
2004\end{array}$ & International & $\begin{array}{l}\text { Matched case- } \\
\text { control }\end{array}$ & $\begin{array}{l}\text { Cases }(n=147) \\
\text { Controls }(n=304)\end{array}$ & Ever use & BRCA1/2 & $0.85(0.53-1.4)$ & $\begin{array}{l}\text { Year of birth, country of } \\
\text { residence, gene }\end{array}$ \\
\hline $\begin{array}{l}\text { Gronwald, } \\
2006\end{array}$ & Europe & $\begin{array}{l}\text { Matched case- } \\
\text { control }\end{array}$ & $\begin{array}{l}\text { Cases }(n=150) \\
\text { Controls }(n=150)\end{array}$ & Ever use & BRCAI & $0.4(0.2-1.0)$ & $\mathrm{n} / \mathrm{a}$ \\
\hline $\begin{array}{l}\text { McLaughlin, } \\
2007\end{array}$ & International & $\begin{array}{l}\text { Matched case- } \\
\text { control }\end{array}$ & $\begin{array}{l}\text { Cases }(n=799) \\
\text { Controls }(n=2,424)\end{array}$ & Ever use & $\begin{array}{l}\text { BRCA1/2 } \\
\text { BRCA1 } \\
\text { BRCA2 } \\
\end{array}$ & $\begin{array}{l}0.53(0.43-0.66) \\
0.56(0.45-0.71) \\
0.39(0.23-0.66) \\
\end{array}$ & $\begin{array}{l}\text { Parity, breastfeeding, oral } \\
\text { contraceptive use, tubal } \\
\text { ligation, ethnicity }\end{array}$ \\
\hline Vicus, 2009 & International & Case-control & $\begin{array}{l}\text { Cases }(\mathrm{n}=154) \\
\text { Controls }(560)\end{array}$ & Ever use & BRCAI & $0.84(0.49-1.44)$ & $\begin{array}{l}\text { Hormone replacement } \\
\text { treatment, parity, year of } \\
\text { birth, age at dx of breast } \\
\text { cancer, radiotherapy, } \\
\text { chemotherapy, type of } \\
\text { breast cancer surgery }\end{array}$ \\
\hline $\begin{array}{l}\text { Antoniou, } \\
2009\end{array}$ & International & $\begin{array}{l}\text { Retrospective } \\
\text { cohort }\end{array}$ & $\begin{array}{l}\text { Cases }(n=253) \\
\text { Controls }(n=3,066)\end{array}$ & Ever use & $\begin{array}{l}\text { BRCA1/2 } \\
\text { BRCA1 } \\
\text { BRCA2 } \\
\end{array}$ & $\begin{array}{l}0.55(0.40-0.76) \\
0.52(0.37-0.73) \\
1.04(0.42-2.54) \\
\end{array}$ & $\begin{array}{l}\text { Number of full-term } \\
\text { pregnancies }\end{array}$ \\
\hline $\begin{array}{l}\text { Kotsopoulos, } \\
2015 \\
\end{array}$ & International & $\begin{array}{l}\text { Matched case- } \\
\text { control }\end{array}$ & $\begin{array}{l}\text { Cases }(n=1,329) \\
\text { Controls }(n=5,267)\end{array}$ & Ever use & $\begin{array}{l}\text { BRCA1 } \\
B R C A 2\end{array}$ & $\begin{array}{l}0.60(0.50-0.71) \\
0.63(0.44-0.92)\end{array}$ & $\begin{array}{l}\text { Age at menarche, parity, } \\
\text { breastfeeding, oral }\end{array}$ \\
\hline
\end{tabular}




\begin{tabular}{|c|c|c|c|c|c|c|c|}
\hline & & & & & & & $\begin{array}{l}\text { contraceptive use, tubal } \\
\text { ligation, ethnicity }\end{array}$ \\
\hline Perri, 2015 & Israel & $\begin{array}{l}\text { Prospective } \\
\text { cohort }\end{array}$ & $\begin{array}{l}\text { Cases }(\mathrm{n}=175) \\
\text { Controls }(\mathrm{n}=898)\end{array}$ & Ever use & $\begin{array}{l}\text { BRCA1/2 } \\
\text { BRCA1 } \\
\text { BRCA2 }\end{array}$ & $\begin{array}{l}0.21(0.14-0.31) \\
0.21(0.14-0.33) \\
0.24(0.09-0.61)\end{array}$ & $\begin{array}{l}\text { Mutation type, age at } \\
\text { menarche, oral } \\
\text { contraceptive use, parity, } \\
\text { age at first pregnancy, use } \\
\text { of hormone replacement } \\
\text { therapy }\end{array}$ \\
\hline $\begin{array}{l}\text { Schrijver, } \\
2021\end{array}$ & International & $\begin{array}{l}\text { Retrospective } \\
\text { cohort }\end{array}$ & $\begin{array}{l}\text { Cases }(n=452) \\
\text { Controls }(n=5,982)\end{array}$ & Ever use & $\begin{array}{l}\text { BRCA1 } \\
\text { BRCA2 } \\
\end{array}$ & $\begin{array}{l}0.51(0.36-0.71) \\
0.65(0.35-1.19) \\
\end{array}$ & $\mathrm{n} / \mathrm{a}$ \\
\hline
\end{tabular}

Odds ratio (OR); hazard ratio (HR) 
The only prospective analysis on oral contraceptive use and risk conducted by Perri et al. recruited 1,073 Jewish Israeli women undergoing fertility treatment between 1995 and $2013^{124}$. Authors reported a similar level of risk reduction for carriers of $B R C A 1$ (HR=0.21; 95\% CI 0.14$0.33)$ and $B R C A 2$ mutations $(\mathrm{HR}=0.24 ; 95 \% \mathrm{CI} 0.09-0.61)$, in addition to an inverse association between increasing duration of use and risk. Nonetheless, the number of women in this study was low, and they did not exclude for prior oophorectomy.

With respect to patterns of oral contraceptive use, two reports based on data from the International BRCA1/2 Carrier Cohort study have investigated associations between year of initiation, age of initiation and time since last use and ovarian cancer ${ }^{75,238}$. The most recent retrospective cohort analysis involved 1,989 BRCA1 mutation carriers and 2,445 BRCA2 mutation carriers ${ }^{238}$. Authors took a left-truncated approach to minimize survival bias and accounted for related aspects of oral contraceptive use. After mutual adjustment, only duration of oral contraceptive use was significantly related to decreased risk of ovarian cancer among BRCA1 mutation carriers $(\mathrm{P}$-trend $=0.008)$. The inverse association between duration of use and risk was stronger for more recent use within the last 15 years $(\mathrm{p}$-trend $=0.0002$ for $<15$ years since last use; $\mathrm{p}$-trend $=0.37$ for $>15 \mathrm{y}$ since last use). Findings were similar for $B R C A 2$ mutation carriers, but confidence intervals were wide (data not published). These results agree with a previous retrospective cohort study based on a subset of the same data. Antoniou et al. also found greater protective effect with longer duration of use and more recent use among BRCA1 mutation carriers ${ }^{75}$.

Collectively, these data demonstrate that oral contraceptives reduce the risk of ovarian cancer by $45 \%$ to $50 \%$ in carriers of a BRCA1 mutation and $50 \%$ in carriers of a BRCA2 mutation $236,238,239$. Moreover, these effects are apparent after relatively short-term use, increase with longer durations of use and persist at least 20 years following cessation of use ${ }^{238}$. 


\subsection{The 'Risk Factor' Study}

Dr. Steven Narod initiated the 'Risk Factor Analysis of Hereditary Breast and Ovarian Cancer' study (i.e. the 'Risk Factor Study') in 1995 based at Women's College Hospital (Toronto, Ontario). The purpose of the study is to identify non-genetic modifiers of breast and ovarian cancer risk in carriers of $B R C A 1$ or BRCA2 mutations. Exposures of interested include reproductive, hormonal and lifestyle factors. Accrual is ongoing as of 2021 across 85 institutions and 17 countries. Currently, the database consists of 17,893 women for whom baseline questionnaire data is available.

All female relatives of mutation-positive families are eligible, and each family may supply more than one study subject. Specifically, inclusion criteria include women who are 1) currently alive, 2) above the age of 18 years and 3) determined to carry a pathogenic mutation in BRCAl and/or BRCA2 by molecular analysis. Women who test negative for a mutation (including variants of unknown significance) and women below 18 years of age are excluded. Eligible participants are approached to enter the study during a genetic counselling appointment at one of the participating centres. 


\section{Chapter 2. Rationale, Objectives and Hypothesis}

\subsection{Rationale}

Women who inherit a deleterious $B R C A 1$ or $B R C A 2$ germline mutation face an increased lifetime risk of developing ovarian and fallopian tube cancer (referred to as ovarian cancer hereafter). Based on prospective studies, the cumulative risk of ovarian cancer to age 80 is estimated between $44 \%$ to $49 \%$ for BRCAl mutation carriers and between $17 \%$ to $21 \%$ for $B R C A 2$ mutation carriers ${ }^{11,12}$. Given the lethality of this disease and lack of adequate screening protocols, primary prevention is strongly recommended. Prophylactic bilateral salpingooophorectomy (i.e., oophorectomy) is the gold standard for risk reduction, associated with a $80 \%$ to $96 \%$ decreased risk of cancer and a $77 \%$ reduction in all-cause mortality among women with a BRCA mutation ${ }^{104,105,240,241}$.

Oral contraceptive use is a well-established protective factor against ovarian cancer among women at high risk due to a BRCA mutation as well as those at baseline population risk ${ }^{210,213,237 .}$ In a recent meta-analysis of six studies conducted among women with a $B R C A 1$ or $B R C A 2$ mutation, Moorman et al. reported that a history of oral contraceptive use was associated with a $42 \%$ reduction in risk $(\mathrm{OR}=0.58 ; 95 \% \mathrm{CI}$ 0.46-0.73) in women with either mutation, consistent with risk estimates observed in the general population ${ }^{237}$. Moreover, this protective effect appears to increase with long-term use and persists over 20 years after discontinuation of use ${ }^{238}$.

The introduction of non-oral LARC methods, including IUDs, implants and injections has caused a considerable shift in patterns of contraceptive use. Globally, 27\% of contraceptive users now rely on LARC methods. The increase in the uptake of IUDs and implants has corresponded to decline in oral contraceptive pill use ${ }^{153}$. LARCs are the most effective and convenient reversible methods of pregnancy prevention with minimal upkeep required by the user. The IUD utilizes a polyethylene, T-shaped frame and releases either copper or a synthetic progestin into the uterine cavity for contraceptive action. The most commonly used hormonal IUD is the five-year $52 \mathrm{mg}$ LNG-bearing IUD, approved in over 140 countries $^{174}$. The most widely distributed nonhormonal IUD is the copper-bearing IUD that contains $200 \mathrm{~mm}^{2}$ to $380 \mathrm{~mm}^{2}$ of exposed copper wire to enhance spermicidal effect. Contraceptive implants consist of progestin-only subdermal 
rods inserted under the skin of the upper arm. Currently available models release either etonorgestrel or LNG, effective for up to five years. Another progestin-only LARC, depot medroxyprogesterone acetate (DMPA), is injected intramuscularly every three months. Similar to oral contraceptives, implants and injections yield higher levels of systemic progestin and primarily prevent pregnancy through inhibition of ovulation ${ }^{242}$. Conversely, IUDs do not consistently suppress ovulation and inhibition of pregnancy is attributed to a foreign body reaction, thickening of the cervical mucus and endometrial atrophy ${ }^{243}$.

Given the documented protective effect oral contraceptive use, it is of interest to investigate whether non-oral contraceptive methods similarly decrease risk of ovarian cancer among women with a $B R C A$ mutation. Data from studies conducted among women in the general population are conflicting. The evidence regarding IUD use and ovarian cancer risk is equivocal, while a mitigating role has been observed with the use of injections and possibly implants $224,227,230,233,234$. To our knowledge, there have been no such studies conducted in the context of BRCA-associated ovarian cancer. As such, the goal of the current study was to evaluate the association between type of contraception and ovarian cancer risk in a cohort of BRCA1 and BRCA2 mutation carriers, accounting for other reproductive and hormonal exposures including prior oral contraceptive use. 


\subsection{Objectives}

1. To describe the prevalence and patterns of use of different types of contraception by birth cohort and BRCA mutation type

2. To evaluate the associations between type of contraception (oral contraceptives, IUDs, implants, injections) and risk of ovarian cancer among $B R C A$ mutation carriers.

3. To evaluate temporal associations between IUD use and risk of ovarian cancer among $B R C A$ mutation carriers.

\subsection{Hypotheses}

1. It is hypothesized that oral contraceptives will be the most popular form of contraception across birth cohorts, but rates of lifetime use will decline in more recent birth cohorts. It is hypothesized that the proportions of IUD, implant and injection use will increase in more recent cohorts of $B R C A$ mutation carriers.

2. It is hypothesized that a history of oral contraceptive, IUD, implant and injection use will be inversely related to ovarian cancer risk among $B R C A$ mutation carriers.

3. It is hypothesized that longer duration of IUD use and later age at first IUD use will be inversely related to ovarian cancer risk among $B R C A$ mutation carriers. 


\section{Chapter 3. Methods}

\subsection{Analysis of Trends in Contraceptive Use in BRCA Mutation Carriers}

\subsubsection{Study Population}

The study population and methods have been previously described in detail ${ }^{244}$. Briefly, eligible subjects included women with a deleterious germline mutation in the BRCA1 or BRCA2 gene who were enrolled in an international, longitudinal study of $B R C A$ mutation carriers from 85 participating centres in 17 countries (see Section Error! Reference source not found.). All women had sought genetic testing due to a personal or family history of breast and/or ovarian cancer. Mutation detection was performed using a range of techniques, but all nucleotide sequences were confirmed by the direct sequencing of DNA. The study protocol was approved by the institutional ethics review board of each participating centre. All subjects provided written informed consent for genetic testing and study participation upon enrolment (see Appendix).

\subsubsection{Data Collection and Storage}

Study subjects completed a standardized baseline research questionnaire at the time of enrollment. Questionnaires were administered by the individual centres either through an inperson interview at the time of their clinic appointment, completed over the phone by a genetic counsellor or research assistant, or mailed to each study participant. The baseline questionnaire collected self-reported information on known or suspected risk factors for breast and ovarian cancer, including personal and/or familial history of cancer, medical history, reproductive history, medication use and selected lifestyle factors. With respect to menopausal status, natural, surgical, medication-induced and other causes for menopause were queried. A follow-up questionnaire was administered every two years thereafter to obtain updated information on exposures, incident cancers, treatments received and vital status. Follow-up questionnaires were either mailed to each participant to complete at home or administered over the phone by a research assistant. The 2018 version of the baseline questionnaire and the 2017 version of the follow-up questionnaire can be found in the Appendix. 
The collection, storage and retrieval of clinical data pertaining to this study is performed at the individual participating centres and forwarded to the study coordinating centre at Women's College Research Institute (Toronto, Canada). The data is checked and entered into the REDCap online database, which is only accessible to study personnel. Physical copies of the research questionnaires, medical release forms and other confidential study materials are locked securely in cabinets.

The current analysis used detailed information regarding history of contraceptive use. In the baseline questionnaire, subjects were asked if they had ever used contraceptives to prevent pregnancy or for any other reason (yes/no) (Figure 4). If 'yes', subjects were prompted to list all start years, stop years, durations of use (years), methods of administration (pill, implant, injection, Mirena/IUD, other) and whether they were current users (yes/no). Starting from the 2004 questionnaire cycle, contraceptive medication name was also collected. The 2002 to 2007 questionnaire cycles only referenced pills, implants and injections as options for method of administration; "Mirena/IUD" was listed as an option since the 2009 questionnaire and 'Other' was an option since the 2016 questionnaire, which allowed women to further specify a method. Updated information on contraceptive use was obtained at each follow-up questionnaire. Specific contraceptive methods that were not included in this analysis include female and male sterilization, withdrawal, natural family planning methods and barrier methods (condom, diaphragm, foam, sponge, suppositories, jelly). 
15a. Have you ever used birth control pills, Norplant (implants), Mirena/IUD, or Depo-Provera (injections) to prevent pregnancy or for any other reason?
$\square$ No $\rightarrow$ Go to question 16.
$\square$ Yes $\rightarrow$ Can you describe the times?

\begin{tabular}{|c|l|l|l|l|l|}
\hline \multicolumn{1}{|c|}{ Method } & Name of Medication & \multicolumn{1}{c|}{$\begin{array}{c}\text { Starting } \\
\text { Year }\end{array}$} & $\begin{array}{c}\text { Ending } \\
\text { Year }\end{array}$ & $\begin{array}{c}\text { Length of Time } \\
\text { Used in Years }\end{array}$ \\
\hline 1 & $\begin{array}{l}\square \text { Pills } \square \text { Implants } \\
\square \text { Mirena/IUD } \\
\square \text { Injections } \square \text { Other }\end{array}$ & & \\
\hline 2 & $\begin{array}{l}\square \text { Pills } \square \text { Implants } \\
\square \text { Mirena/IUD } \\
\square \text { Injections } \square \text { Other }\end{array}$ & & & \\
\hline 3 & $\begin{array}{l}\square \text { Pills } \square \text { Implants } \\
\square \text { Mirena/IUD } \\
\square \text { Injections } \square \text { Other }\end{array}$ & & & \\
\hline 4 & $\begin{array}{l}\square \text { Pills } \square \text { Implants } \\
\square \text { Mirena/IUD } \\
\square \text { Injections } \square \text { Other }\end{array}$ & & & \\
\hline
\end{tabular}

15b. Are you currently using birth control pills, Norplant (implants), Mirena/IUD, or Depo-Provera (injections)?

$\square \quad$ No

Yes

Figure 4. Contraceptive use questions included in the baseline research questionnaire for the Risk Factor Analysis of Breast and Ovarian Cancer (Version: 22 May 2018) 


\subsubsection{Study Subjects Available for Analysis}

Study subjects were derived from an exploratory, prospective evaluation of contraceptive use and ovarian cancer risk. A total of 17,694 $\mathrm{BRCA}$ mutation carriers were available for inclusion in this study. Women were excluded if they had a prevalent cancer at baseline or a missing personal history of cancer $(n=10,833)$; no follow up information $(n=2,171)$; no date of birth, baseline date or date of death $(n=590)$; bilateral oophorectomy prior to baseline $(n=777)$; missing information on birth control use over follow-up period $(n=4)$; or missing $B R C A$ mutation type $(n=36)$. After exclusions, a total of 3,283 $\mathrm{BRCA}$ mutation carriers were available for analysis, including 2,254 women who reported a history of contraceptive use (

Figure 5). The results of the prospective analysis were underpowered and will not be presented; however, data from the 2,254 contraceptive users were included in a descriptive study of patterns of contraceptive use by birth cohort and BRCA mutation type.

Excluded from analysis $(n=14,411)$

1. Prevalent cancer at baseline or missing personal history of cancer $(n=10,833)$

2. No follow up information $(n=2,171)$

3. No date of birth, baseline date or date of death $(n=590)$

4. Bilateral oophorectomy prior to baseline $(n=777)$

5. Missing information on birth control use over follow-up period $(n=4)$

6. Missing $B R C A$ mutation type $(\mathrm{n}=36)$

Recruitment 1996-2020 ( $n=17,694)$

- Inclusion criteria: $B R C A 1 / 2$ mutation carrier, $\geq 18$ years

Eligible cohort $(n=3,283)$

- History of contraceptive use $(n=2,254)$

Figure 5. Recruitment statistics for a prospective cohort study of contraceptive use and ovarian cancer risk among $B R C A$ mutation carriers 


\subsubsection{Data Cleaning}

Due to limitations of the earlier Microsoft Access database, baseline data on contraceptive method and duration of use, as well as follow-up data on medication name and year of last use were not historically entered. To address missing information, we cleaned the data pertaining to contraceptive use for 2,254 contraceptive users (20.20\% of all identified contraceptive users) identified for an exploratory, prospective analysis (data not presented). Participant file folders were retrieved, and the content of the physical baseline and follow-up questionnaires was crosschecked against information on the REDCap database. Given the variety of brand name and generic products that represent therapeutically equivalent formulations, misclassification of the 'medication name' variable was another issue during the data cleaning process. Table 8 shows the hormonal contraception identification chart created as a reference for coding patient self-reported medication names against 60 categorical response options available on REDCap. Specific options include 54 brand names and six umbrella terms ("unknown”, "other pill”, “implant", "injection", "IUD”, "more than one"). To address inconsistencies in self-reporting, participants who reported never-use of any contraception, but provided details of contraceptive use were corrected as ever-users. Participants who reported ever-use of contraception but failed to identify a medication name or method were categorized as 'missing'. 


\section{Table 8. REDCap hormonal contraception identification chart}

\begin{tabular}{|c|c|c|c|c|c|c|c|c|}
\hline $\begin{array}{l}\text { REDCap } \\
\text { options }\end{array}$ & Method & Equivalent drug names & Cycle & Days & $\begin{array}{l}\text { Estrogen } \\
\text { type }\end{array}$ & $\begin{array}{l}\text { Estrogen } \\
\text { dose }(\mathrm{mg})\end{array}$ & Progestin type & $\begin{array}{l}\text { Progestin } \\
\text { dose (mg) }\end{array}$ \\
\hline \multirow[t]{2}{*}{ Demulen 50} & \multirow[t]{2}{*}{$\begin{array}{l}\text { Combined oral } \\
\text { contraceptive }\end{array}$} & \multirow[t]{2}{*}{ Kelnor $1 / 50$, Zovia $1 / 50$} & \multirow[t]{2}{*}{ Monophasic } & 1 to 21 & $\begin{array}{l}\text { Ethinyl } \\
\text { estradiol }\end{array}$ & 0.05 & Ethynodiol diacetate & 1 \\
\hline & & & & 22 to 28 & - & - & - & - \\
\hline \multirow{2}{*}{$\begin{array}{l}\text { Norinyl/ } \\
\text { Ortho- } \\
\text { Novum 1:50 }\end{array}$} & \multirow{2}{*}{$\begin{array}{l}\text { Combined oral } \\
\text { contraceptive }\end{array}$} & \multirow{2}{*}{$\begin{array}{l}\text { Genora } 1 / 50, \text { Necon } 1 / 50, \\
\text { Nelova } 1 / 50, \text { Norethin } 1 / 50 \text {, } \\
\text { Nortrel } 1 / 50\end{array}$} & \multirow[t]{2}{*}{ Monophasic } & 1 to 21 & Mestranol & 0.05 & Norethindrone & 1 \\
\hline & & & & 22 to 28 & - & - & - & - \\
\hline \multirow[t]{2}{*}{ Ovral } & \multirow[t]{2}{*}{$\begin{array}{l}\text { Combined oral } \\
\text { contraceptive }\end{array}$} & & \multirow[t]{2}{*}{ Monophasic } & 1 to 21 & $\begin{array}{l}\text { Ethinyl } \\
\text { estradiol }\end{array}$ & 0.05 & Norgestrel & 0.5 \\
\hline & & & & 22 to 28 & - & - & - & - \\
\hline \multirow[t]{2}{*}{$\begin{array}{l}\text { Brevicon/ } \\
\text { Ortho 0.5:35 }\end{array}$} & \multirow[t]{2}{*}{$\begin{array}{l}\text { Combined oral } \\
\text { contraceptive }\end{array}$} & \multirow{2}{*}{$\begin{array}{l}\text { Cyclafem } 0.5 / 35 \text {, Cyonanz, } \\
\text { Modicon, Necon 0.5/35, } \\
\text { Nortrel 0.5/35, Ortho 0.5/35, } \\
\text { Ovysmen, Wera }\end{array}$} & \multirow[t]{2}{*}{ Monophasic } & 1 to 21 & $\begin{array}{l}\text { Ethinyl } \\
\text { estradiol }\end{array}$ & 0.035 & Norethindrone & 0.5 \\
\hline & & & & 22 to 28 & - & - & - & - \\
\hline \multirow{2}{*}{$\begin{array}{l}\text { Brevicon/ } \\
\text { Ortho/ Select } \\
1: 35\end{array}$} & \multirow[t]{2}{*}{$\begin{array}{l}\text { Combined oral } \\
\text { contraceptive }\end{array}$} & \multirow{2}{*}{$\begin{array}{l}\text { Alyacen } 1 / 35 \text {, Brevicon } \\
1 / 35 \text {, Cyclafem } 1 / 35, \\
\text { Dasetta } 1 / 35 \text {, Necon } 1 / 35 \text {, } \\
\text { Norethin } 1 / 25 \text {, Norinyl } 1 / 35 \text {, } \\
\text { Nortrel } 1 / 35 \text {, Nylia } 1 / 35 \text {, } \\
\text { Ortho-Novum } 1 / 35 \text {, } \\
\text { Pirmella } 1 / 35 \text {, Select } 1 / 35\end{array}$} & \multirow[t]{2}{*}{ Monophasic } & 1 to 21 & $\begin{array}{l}\text { Ethinyl } \\
\text { estradiol }\end{array}$ & 0.035 & Norethindrone & 1 \\
\hline & & & & 22 to 28 & - & - & - & - \\
\hline \multirow[t]{2}{*}{ Cyclen 35} & \multirow[t]{2}{*}{$\begin{array}{l}\text { Combined oral } \\
\text { contraceptive }\end{array}$} & \multirow{2}{*}{$\begin{array}{l}\text { Cilest, Estarylla, Mili, } \\
\text { Mono-Linyah, MonoNessa, } \\
\text { Ortho-Cyclen, Previfem, } \\
\text { Sprintec }\end{array}$} & \multirow[t]{2}{*}{ Monophasic } & 1 to 21 & $\begin{array}{l}\text { Ethinyl } \\
\text { estradiol }\end{array}$ & 0.035 & Norgestimate & 0.25 \\
\hline & & & & 22 to 28 & - & - & - & - \\
\hline \multirow[t]{2}{*}{ Demulen 30} & \multirow[t]{2}{*}{$\begin{array}{l}\text { Combined oral } \\
\text { contraceptive }\end{array}$} & & \multirow[t]{2}{*}{ Monophasic } & 1 to 21 & $\begin{array}{l}\text { Ethinyl } \\
\text { estradiol }\end{array}$ & 0.03 & Ethynodiol diacetate & 1 \\
\hline & & & & 22 to 28 & - & - & - & - \\
\hline \multirow[t]{2}{*}{ Loestrin 30} & \multirow[t]{2}{*}{$\begin{array}{l}\text { Combined oral } \\
\text { contraceptive }\end{array}$} & \multirow{2}{*}{$\begin{array}{l}\text { Aurovela } 1.5 / 30 \text {, Blisovi Fe } \\
1.5 / 30 \text {, Gildess } 1.5 / 30, \\
\text { Hailey } 1.5 / 30 \text {, Junel } 1.5 / 30 \text {, } \\
\text { Larin } 1.5 / 30 \text {, Loestrin } \\
1.5 / 30 \text {, Microgestin } 1.5 / 30\end{array}$} & \multirow[t]{2}{*}{ Monophasic } & 1 to 21 & $\begin{array}{l}\text { Ethinyl } \\
\text { estradiol }\end{array}$ & 0.03 & Norethindrone acetate & 1.5 \\
\hline & & & & 22 to 28 & - & - & - & - \\
\hline
\end{tabular}




\begin{tabular}{|c|c|c|c|c|c|c|c|c|}
\hline \multirow[t]{2}{*}{$\begin{array}{l}\text { Min-Orval } \\
30\end{array}$} & \multirow[t]{2}{*}{$\begin{array}{l}\text { Combined oral } \\
\text { contraceptive }\end{array}$} & \multirow{2}{*}{$\begin{array}{l}\text { Altavera, Ayuna, Chateal, } \\
\text { Indayo, Jolessa, Kurvelo, } \\
\text { Levlen, Levora } 15 / 30, \\
\text { Marlissa, Microgynon } 30 \text {, } \\
\text { Nordette, Ovima, Portia }\end{array}$} & \multirow[t]{2}{*}{ Monophasic } & 1 to 21 & $\begin{array}{l}\text { Ethinyl } \\
\text { estradiol }\end{array}$ & 0.03 & Levonorgestrel & 0.15 \\
\hline & & & & 22 to 28 & - & - & - & - \\
\hline \multirow{2}{*}{$\begin{array}{l}\text { Marvelon/ } \\
\text { Ortho-Cept } \\
30\end{array}$} & \multirow[t]{2}{*}{$\begin{array}{l}\text { Combined oral } \\
\text { contraceptive }\end{array}$} & \multirow{2}{*}{$\begin{array}{l}\text { Apri, Desogen, Emoquette, } \\
\text { Enskyce, Isibloom, Kalliga, } \\
\text { Marvelon, Ortho-Cept, } \\
\text { Reclipsen, Solia } \\
\end{array}$} & \multirow[t]{2}{*}{ Monophasic } & 1 to 21 & $\begin{array}{l}\text { Ethinyl } \\
\text { estradiol }\end{array}$ & 0.03 & Desogestrel & 0.15 \\
\hline & & & & 22 to 28 & - & - & - & - \\
\hline \multirow[t]{2}{*}{ Alesse } & \multirow[t]{2}{*}{$\begin{array}{l}\text { Combined oral } \\
\text { contraceptive }\end{array}$} & \multirow{2}{*}{$\begin{array}{l}\text { Afirmelle, Alesse, Alysena, } \\
\text { Aubra, Aviane, Balcoltra, } \\
\text { Delyla, Falmina, Larissia, } \\
\text { Lutera, Levlite, Lessina, } \\
\text { Orsythia, Sronynx, Vienva, } \\
\text { Minisiston }\end{array}$} & \multirow[t]{2}{*}{ Monophasic } & 1 to 21 & $\begin{array}{l}\text { Ethinyl } \\
\text { estradiol }\end{array}$ & 0.02 & Levonorgestrel & 0.1 \\
\hline & & & & 22 to 28 & - & - & - & - \\
\hline \multirow[t]{4}{*}{ Minestrin 20} & \multirow[t]{4}{*}{$\begin{array}{l}\text { Combined oral } \\
\text { contraceptive }\end{array}$} & \multirow{2}{*}{$\begin{array}{l}\text { Aurovela (Fe) } 1 / 20, \text { Blisovi } \\
\text { Fe } 1 / 20, \text { Gildess }(\mathrm{Fe}) 1 / 20 . \\
\text { Hailey Fe } 1 / 20, \text { Junel }(\mathrm{Fe}) \\
\text { 1/20, Larin }(\mathrm{Fe}) 1 / 20, \\
\text { Loestrin }(\mathrm{Fe}) 1 / 20, \\
\text { Microgestin } 1 / 20, \text { Tarina } \\
(\mathrm{Fe}) 1 / 20\end{array}$} & \multirow[t]{2}{*}{ Monophasic } & 1 to 21 & $\begin{array}{l}\text { Ethinyl } \\
\text { estradiol }\end{array}$ & 0.02 & Norethindrone acetate & 1 \\
\hline & & & & 22 to 28 & - & - & - & - \\
\hline & & \multirow{2}{*}{$\begin{array}{l}\text { Aurovela } 24 \text { FE, Blisovi } 24 \\
\text { FE. Finzala, Gildess } 24 \text { FE, } \\
\text { Larin } 24 \text { FE, Loestrin } 24 \\
\text { FE, Lomedia } 24 \text { FE, } \\
\text { Mibelas } 24 \text { FE, Minastrin } 24 \\
\text { FE, Taytulla }{ }^{\circledR}\end{array}$} & Monophasic & 1 to 24 & $\begin{array}{l}\text { Ethinyl } \\
\text { estradiol }\end{array}$ & 0.02 & Norethindrone acetate & 1 \\
\hline & & & & 25 to 28 & - & - & - & \\
\hline Micronor & $\begin{array}{l}\text { Progestin-only } \\
\text { (pill) }\end{array}$ & $\begin{array}{l}\text { Aygestin, Camilla, Errin, } \\
\text { Jolivette, Nora-BE, Nor-QD }\end{array}$ & Monophasic & 1 to 28 & - & - & Norethindrone & 0.35 \\
\hline \multirow[t]{3}{*}{ Ortho $10 / 11$} & \multirow[t]{3}{*}{$\begin{array}{l}\text { Combined oral } \\
\text { contraceptive }\end{array}$} & \multirow[t]{3}{*}{$\begin{array}{l}\text { Necon } 10 / 11, \text { Ortho-novum } \\
\text { 10/11 (Discontinued) }\end{array}$} & \multirow[t]{3}{*}{ Biphasic } & 1 to 10 & $\begin{array}{l}\text { Ethinyl } \\
\text { estradiol }\end{array}$ & 0.035 & Norethindrone & 0.5 \\
\hline & & & & 11 to 21 & $\begin{array}{l}\text { Ethinyl } \\
\text { estradiol }\end{array}$ & 0.035 & Norethindrone & 1 \\
\hline & & & & 22 to 28 & - & - & - & - \\
\hline \multirow[t]{2}{*}{ Ortho $7 / 7 / 7$} & \multirow[t]{2}{*}{$\begin{array}{l}\text { Combined oral } \\
\text { contraceptive }\end{array}$} & \multirow{2}{*}{$\begin{array}{l}\text { Alyacen } 7 / 7 / 7, \text { Cyclafem } \\
7 / 7 / 7, \text { Dasetta 7/7/7, Necon } \\
7 / 7 / 7, \text { Nortrel 7/7/7, }\end{array}$} & \multirow[t]{2}{*}{ Triphasic } & 1 to 7 & $\begin{array}{l}\text { Ethinyl } \\
\text { estradiol }\end{array}$ & 0.035 & Norethindrone & 0.5 \\
\hline & & & & 8 to 14 & $\begin{array}{l}\text { Ethinyl } \\
\text { estradiol }\end{array}$ & 0.035 & Norethindrone & 0.75 \\
\hline
\end{tabular}




\begin{tabular}{|c|c|c|c|c|c|c|c|c|}
\hline & & \multirow[t]{2}{*}{$\begin{array}{l}\text { NyliaTM 7/7/7, Ortho- } \\
\text { Novum 7/7/7, Pirmella 7/7/7 }\end{array}$} & & 15 to 21 & $\begin{array}{l}\text { Ethinyl } \\
\text { estradiol }\end{array}$ & 0.035 & Norethindrone & 1 \\
\hline & & & & 22 to 28 & - & - & - & - \\
\hline \multirow[t]{5}{*}{ Synphasic } & \multirow[t]{5}{*}{$\begin{array}{l}\text { Combined oral } \\
\text { contraceptive }\end{array}$} & & \multirow[t]{2}{*}{ Biphasic } & 1 to 9 & $\begin{array}{l}\text { Ethinyl } \\
\text { estradiol }\end{array}$ & 0.035 & Norethindrone & 1 \\
\hline & & & & 10 to 21 & $\begin{array}{l}\text { Ethinyl } \\
\text { estradiol }\end{array}$ & 0.035 & Norethindrone & 0.5 \\
\hline & & & \multirow[t]{3}{*}{ Biphasic } & 1 to 9 & $\begin{array}{l}\text { Ethinyl } \\
\text { estradiol }\end{array}$ & 0.035 & Norethindrone & 1 \\
\hline & & & & 10 to 21 & $\begin{array}{l}\text { Ethinyl } \\
\text { estradiol }\end{array}$ & 0.035 & Norethindrone & 0.5 \\
\hline & & & & 22 to 28 & - & - & - & - \\
\hline \multirow[t]{7}{*}{ Tri-Cyclen } & \multirow[t]{7}{*}{$\begin{array}{l}\text { Combined oral } \\
\text { contraceptive }\end{array}$} & \multirow{7}{*}{$\begin{array}{l}\text { Ortho-Tricyclen, TriNessa, } \\
\text { Tricyclen } \\
\text { Tri-Cyclen Lo, Ortho Tri- } \\
\text { cyclen Lo, Tri-Lo-Sprintec }\end{array}$} & \multirow[t]{3}{*}{ Triphasic } & 1 to 7 & $\begin{array}{l}\text { Ethinyl } \\
\text { estradiol }\end{array}$ & 0.035 & Norgestimate & 0.18 \\
\hline & & & & 8 to 14 & $\begin{array}{l}\text { Ethinyl } \\
\text { estradiol }\end{array}$ & 0.035 & Norgestimate & 0.215 \\
\hline & & & & 15 to 21 & $\begin{array}{l}\text { Ethinyl } \\
\text { estradiol }\end{array}$ & 0.035 & Norgestimate & 0.25 \\
\hline & & & \multirow[t]{4}{*}{ Biphasic } & 1 to 7 & $\begin{array}{l}\text { Ethinyl } \\
\text { estradiol }\end{array}$ & 0.035 & Norgestimate & 0.18 \\
\hline & & & & 8 to 14 & $\begin{array}{l}\text { Ethinyl } \\
\text { estradiol }\end{array}$ & 0.035 & Norgestimate & 0.215 \\
\hline & & & & 15 to 21 & $\begin{array}{l}\text { Ethinyl } \\
\text { estradiol }\end{array}$ & 0.035 & Norgestimate & 0.25 \\
\hline & & & & 22 to 28 & - & - & - & - \\
\hline \multirow[t]{6}{*}{$\begin{array}{l}\text { Triphasil/ } \\
\text { Triquilar }\end{array}$} & \multirow[t]{6}{*}{$\begin{array}{l}\text { Combined oral } \\
\text { contraceptive }\end{array}$} & \multirow{6}{*}{$\begin{array}{l}\text { Trionetta, Trigynon, } \\
\text { Logynon, Trivora, Trilevlen, } \\
\text { Elifemme, Enpresse, } \\
\text { Levonest, Myzilra }\end{array}$} & \multirow[t]{3}{*}{ Triphasic } & 1 to 7 & $\begin{array}{l}\text { Ethinyl } \\
\text { estradiol }\end{array}$ & 0.03 & Levonorgestrel & 0.05 \\
\hline & & & & 8 to 14 & $\begin{array}{l}\text { Ethinyl } \\
\text { estradiol }\end{array}$ & 0.04 & Levonorgestrel & 0.075 \\
\hline & & & & 15 to 21 & $\begin{array}{l}\text { Ethinyl } \\
\text { estradiol }\end{array}$ & 0.03 & Levonorgestrel & 0.125 \\
\hline & & & \multirow[t]{3}{*}{ Triphasic } & 1 to 7 & $\begin{array}{l}\text { Ethinyl } \\
\text { estradiol }\end{array}$ & 0.03 & Levonorgestrel & 0.05 \\
\hline & & & & 8 to 14 & $\begin{array}{l}\text { Ethinyl } \\
\text { estradiol }\end{array}$ & 0.04 & Levonorgestrel & 0.075 \\
\hline & & & & 15 to 21 & $\begin{array}{l}\text { Ethinyl } \\
\text { estradiol }\end{array}$ & 0.03 & Levonorgestrel & 0.125 \\
\hline
\end{tabular}




\begin{tabular}{|c|c|c|c|c|c|c|c|}
\hline & & & 22 to 28 & - & - & - & - \\
\hline \multirow[t]{21}{*}{ Other Pill } & \multirow[t]{3}{*}{ Lolo, Lo Loestrin Fe } & \multirow[t]{3}{*}{ Biphasic } & 1 to 24 & $\begin{array}{l}\text { Ethinyl } \\
\text { estradiol }\end{array}$ & 0.01 & Norethindrone acetate & 1 \\
\hline & & & 25 to 26 & $\begin{array}{l}\text { Ethinyl } \\
\text { estradiol }\end{array}$ & 0.01 & - & - \\
\hline & & & 27 to 28 & $\mathrm{Fe}$ & 75 & - & - \\
\hline & \multirow[t]{2}{*}{$\begin{array}{l}\text { Generess Fe, Kaitlib Fe, } \\
\text { Layolis Fe }\end{array}$} & \multirow[t]{2}{*}{ Monophasic } & 1 to 24 & $\begin{array}{l}\text { Ethinyl } \\
\text { estradiol }\end{array}$ & 0.025 & Norethindrone & 0.8 \\
\hline & & & 25 to 28 & $\mathrm{Fe}$ & 75 & - & - \\
\hline & \multirow[t]{2}{*}{$\begin{array}{l}\text { Demulen } 1 / 35 \text {, Kelnor } 1 / 35 \text {, } \\
\text { Zovia } 1 / 35\end{array}$} & & 1 to 21 & $\begin{array}{l}\text { Ethinyl } \\
\text { estradiol }\end{array}$ & 0.035 & Ethynodiol diacetate & 1 \\
\hline & & & 22 to 28 & - & - & - & - \\
\hline & \multirow[t]{2}{*}{ Tilia Fe } & \multirow[t]{2}{*}{ Biphasic } & 1 to 21 & $\begin{array}{l}\text { Ethinyl } \\
\text { estradiol }\end{array}$ & 0.02 & Norethindrone acetate & 1 \\
\hline & & & 22 to 28 & $\mathrm{Fe}$ & 75 & & \\
\hline & \multirow[t]{4}{*}{ Estrostep Fe, Tri-Legest Fe } & \multirow[t]{4}{*}{ Triphasic } & 1 to 7 & $\begin{array}{l}\text { Ethinyl } \\
\text { estradiol }\end{array}$ & 0.02 & Norethindrone acetate & 1 \\
\hline & & & 8 to 14 & $\begin{array}{l}\text { Ethinyl } \\
\text { estradiol }\end{array}$ & 0.03 & Norethindrone acetate & 1 \\
\hline & & & 15 to 21 & $\begin{array}{l}\text { Ethinyl } \\
\text { estradiol }\end{array}$ & 0.035 & Norethindrone acetate & 1 \\
\hline & & & 22 to 28 & $\mathrm{Fe}$ & 75 & & \\
\hline & \multirow[t]{2}{*}{$\begin{array}{l}\text { Lo-Ovral, Chryselle, } \\
\text { Elinest, Low-Ogestrel }\end{array}$} & \multirow[t]{2}{*}{ Monophasic } & 1 to 21 & $\begin{array}{l}\text { Ethinyl } \\
\text { estradiol }\end{array}$ & 0.03 & Norgestrel & 0.3 \\
\hline & & & 22 to 28 & - & - & - & - \\
\hline & \multirow[t]{2}{*}{ LoSeasonique } & \multirow[t]{2}{*}{ Monophasic } & 1 to 84 & $\begin{array}{l}\text { Ethinyl } \\
\text { estradiol }\end{array}$ & 0.02 & Levonorgestrel & 0.1 \\
\hline & & & 85 to 91 & $\begin{array}{l}\text { Ethinyl } \\
\text { estradiol }\end{array}$ & 0.01 & & \\
\hline & Ovrette & & Daily & - & - & Norgestrel & 0.075 \\
\hline & \multirow[t]{3}{*}{ Qlaira } & \multirow[t]{3}{*}{ Quadriphasic } & 1 to 2 & $\begin{array}{l}\text { Estradiol } \\
\text { valerate }\end{array}$ & 3 & - & - \\
\hline & & & 3 to 7 & $\begin{array}{l}\text { Estradiol } \\
\text { valerate }\end{array}$ & 2 & Dienogest & 2 \\
\hline & & & 8 to 24 & $\begin{array}{l}\text { Estradiol } \\
\text { valerate }\end{array}$ & 2 & Dienogest & 3 \\
\hline
\end{tabular}




\begin{tabular}{|c|c|c|c|c|c|c|c|c|}
\hline & & & & 25 to 26 & $\begin{array}{l}\text { Estradiol } \\
\text { valerate }\end{array}$ & 1 & - & - \\
\hline & & & & 27 to 28 & - & - & - & - \\
\hline & & Gravistat & Monophasic & 1 to 21 & $\begin{array}{l}\text { Ethinyl } \\
\text { estradiol }\end{array}$ & 0.05 & Levonorgestrel & 0.125 \\
\hline & & & & 22 to 28 & - & - & - & - \\
\hline & & Angravid & & & Mestranol & 0.05 & Ethynodiol diacetate & 1 \\
\hline & & Azurette, Mircette, Kariva & Biphasic & 1 to 21 & $\begin{array}{l}\text { Ethinyl } \\
\text { estradiol }\end{array}$ & 0.02 & Desogestrel & 0.15 \\
\hline & & & & 22 to 23 & - & - & - & - \\
\hline & & & & 24 to 28 & $\begin{array}{l}\text { Ethinyl } \\
\text { estradiol }\end{array}$ & 0.01 & - & - \\
\hline & & $\begin{array}{l}\text { Cyclessa, Cesia, Caziant, } \\
\text { Velivet, Linessa }\end{array}$ & Triphasic & 1 to 7 & $\begin{array}{l}\text { Ethinyl } \\
\text { estradiol }\end{array}$ & 0.025 & Desogestrel & 0.1 \\
\hline & & & & 8 to 14 & $\begin{array}{l}\text { Ethinyl } \\
\text { estradiol }\end{array}$ & 0.025 & Desogestrel & 0.125 \\
\hline & & & & 9 to 21 & $\begin{array}{l}\text { Ethinyl } \\
\text { estradiol }\end{array}$ & 0.025 & Desogestrel & 0.15 \\
\hline & & & & 22 to 28 & - & - & - & - \\
\hline Injection & Injectable & Noristerat & 2 months & & - & - & $\begin{array}{l}\text { Norethisterone } \\
\text { enantate }\end{array}$ & 200 \\
\hline & & Sayana Press & 13 weeks & & - & - & $\begin{array}{l}\text { Medroxyprogesterone } \\
\text { acetate }\end{array}$ & 104 \\
\hline & & Lunelle & Monthly & & $\begin{array}{l}\text { Estradiol } \\
\text { cypionate }\end{array}$ & 5 & $\begin{array}{l}\text { Medroxyprogesterone } \\
\text { acetate }\end{array}$ & 25 \\
\hline Implants & Progestin-only & Norplant & & & & & Levonorgestrel & 216 \\
\hline & (implant) & Jadelle & & & & & Levonorgestrel & 150 \\
\hline Logest & $\begin{array}{l}\text { Combined oral } \\
\text { contraceptive }\end{array}$ & Meliane, Sylvie 20 & Monophasic & 1 to 21 & $\begin{array}{l}\text { Ethinyl } \\
\text { estradiol }\end{array}$ & 0.02 & Gestodene & 0.075 \\
\hline Trionetta & $\begin{array}{l}\text { Combined oral } \\
\text { contraceptive }\end{array}$ & $\begin{array}{l}\text { Triquilar, Triphasil, } \\
\text { Trigynon, Logynon, }\end{array}$ & Triphasic & 1 to 7 & $\begin{array}{l}\text { Ethinyl } \\
\text { estradiol }\end{array}$ & 0.03 & Levonorgestrel & 0.05 \\
\hline & & $\begin{array}{l}\text { Trivora, Trilevlen, } \\
\text { Elifemme, Enpresse, }\end{array}$ & & 8 to 14 & $\begin{array}{l}\text { Ethinyl } \\
\text { estradiol }\end{array}$ & 0.04 & Levonorgestrel & 0.075 \\
\hline & & Levonest, Myzilra & & 15 to 21 & $\begin{array}{l}\text { Ethinyl } \\
\text { estradiol }\end{array}$ & 0.03 & Levonorgestrel & 0.125 \\
\hline & & & & 1 to 7 & $\begin{array}{l}\text { Ethinyl } \\
\text { estradiol }\end{array}$ & 0.03 & Levonorgestrel & 0.05 \\
\hline
\end{tabular}




\begin{tabular}{|c|c|c|c|c|c|c|c|c|}
\hline & & & & 8 to 14 & $\begin{array}{l}\text { Ethinyl } \\
\text { estradiol }\end{array}$ & 0.04 & Levonorgestrel & 0.075 \\
\hline & & & & 15 to 21 & $\begin{array}{l}\text { Ethinyl } \\
\text { estradiol }\end{array}$ & 0.03 & Levonorgestrel & 0.125 \\
\hline & & & & 22 to 28 & - & - & - & - \\
\hline Diane 35 & $\begin{array}{l}\text { Combined oral } \\
\text { contraceptive }\end{array}$ & Cleo-35, Cyestra-35 & Monophasic & 1 to 21 & $\begin{array}{l}\text { Ethinyl } \\
\text { estradiol }\end{array}$ & 0.035 & Cyproterone acetate & 2 \\
\hline \multirow[t]{3}{*}{ Yasmin } & \multirow[t]{3}{*}{$\begin{array}{l}\text { Combined oral } \\
\text { contraceptive }\end{array}$} & \multirow{3}{*}{$\begin{array}{l}\text { Qismette, Zamine, Zarah, } \\
\text { Ocella, Yaela, Syeda, } \\
\text { Safyral }\end{array}$} & \multirow[t]{3}{*}{ Monophasic } & 1 to 21 & $\begin{array}{l}\text { Ethinyl } \\
\text { estradiol }\end{array}$ & 0.03 & Drospirenone & 3 \\
\hline & & & & 22 to 28 & - & - & - & - \\
\hline & & & & 1 to 21 & $\begin{array}{l}\text { Ethinyl } \\
\text { estradiol }\end{array}$ & 0.03 & Drospirenone & 3 \\
\hline $\begin{array}{l}\text { Depo- } \\
\text { provera }\end{array}$ & Injectable & & Monophasic & $\begin{array}{l}\text { Every } 3 \\
\text { months }\end{array}$ & - & - & $\begin{array}{l}\text { Medroxyprogesterone } \\
\text { acetate }\end{array}$ & 150 \\
\hline Meliane & $\begin{array}{l}\text { Combined oral } \\
\text { contraceptive }\end{array}$ & Logest, Sylvie 20, Harmonet & Monophasic & 1 to 21 & $\begin{array}{l}\text { Ethinyl } \\
\text { estradiol }\end{array}$ & 0.02 & Gestodene & 0.075 \\
\hline Orgametril & $\begin{array}{l}\text { Progestin-only } \\
\text { (pill) }\end{array}$ & Exluton, Exlutona & Monophasic & 1 to 30 & - & - & Lynestronol & 5 \\
\hline Cilest & $\begin{array}{l}\text { Combined oral } \\
\text { contraceptive }\end{array}$ & $\begin{array}{l}\text { Cilest 35/250, Cyclen } 35 \text {, } \\
\text { Sprintec }\end{array}$ & Monophasic & 1 to 21 & $\begin{array}{l}\text { Ethinyl } \\
\text { estradiol }\end{array}$ & 0.035 & Norgestimate & 0.25 \\
\hline Patentex & $\begin{array}{l}\text { Vaginal } \\
\text { suppository } \\
\text { (spermicide) } \\
\end{array}$ & Patentex Oval & Monophasic & - & Nonoxynol & 75 & - & - \\
\hline $\begin{array}{l}\text { Harmonet } \\
\text { \{Europe }\end{array}$ & $\begin{array}{l}\text { Combined oral } \\
\text { contraceptive }\end{array}$ & Logest, Sylvie 20, Meliane & Monophasic & 1 to 21 & $\begin{array}{l}\text { Ethinyl } \\
\text { estradiol }\end{array}$ & 0.02 & Gestodene & 0.075 \\
\hline \multirow[t]{3}{*}{ Tri-Regol } & \multirow[t]{3}{*}{$\begin{array}{l}\text { Combined oral } \\
\text { contraceptive }\end{array}$} & \multirow{3}{*}{$\begin{array}{l}\text { Fironetta, Levordiol, } \\
\text { Logynon, Modutrol, } \\
\text { Triagynon, Triciclor, } \\
\text { Triette, Trigoa, Trigynon, } \\
\text { Trikvilar, Tri-Levlen, } \\
\text { Trinordiol, Trionetta, } \\
\text { Triovlar, Triphasil, } \\
\text { Triquilar, Trisiston, } \\
\text { TriStediril, Trolit }\end{array}$} & \multirow[t]{3}{*}{ Triphasic } & 1 to 7 & $\begin{array}{l}\text { Ethinyl } \\
\text { estradiol }\end{array}$ & 0.03 & Levonorgestrel & 0.05 \\
\hline & & & & 8 to 14 & $\begin{array}{l}\text { Ethinyl } \\
\text { estradiol }\end{array}$ & 0.04 & Levonorgestrel & 0.075 \\
\hline & & & & 15 to 21 & $\begin{array}{l}\text { Ethinyl } \\
\text { estradiol }\end{array}$ & 0.03 & Levonorgestrel & 0.125 \\
\hline Mercilon & $\begin{array}{l}\text { Combined oral } \\
\text { contraceptive }\end{array}$ & $\begin{array}{l}\text { Novynette, Desmin 20, } \\
\text { Femina }\end{array}$ & Monophasic & 1 to 21 & $\begin{array}{l}\text { Ethinyl } \\
\text { estradiol }\end{array}$ & 0.02 & Desogestrel & 0.15 \\
\hline
\end{tabular}




\begin{tabular}{|c|c|c|c|c|c|c|c|c|}
\hline & & & & 1 to 21 & $\begin{array}{l}\text { Ethinyl } \\
\text { estradiol }\end{array}$ & 0.02 & Desogestrel & 0.15 \\
\hline & & & & 22 to 28 & - & - & - & - \\
\hline \multirow[t]{3}{*}{ Desogen } & \multirow[t]{3}{*}{$\begin{array}{l}\text { Combined oral } \\
\text { contraceptive }\end{array}$} & \multirow{3}{*}{$\begin{array}{l}\text { Marvelon, Ortho-Cept, Apri, } \\
\text { Emoquette, Enskyce, } \\
\text { Isibloom, Reclipsen, Solia }\end{array}$} & \multirow[t]{3}{*}{ Monophasic } & 1 to 21 & $\begin{array}{l}\text { Ethinyl } \\
\text { estradiol }\end{array}$ & 0.03 & Desogestrel & 0.15 \\
\hline & & & & 1 to 21 & $\begin{array}{l}\text { Ethinyl } \\
\text { estradiol }\end{array}$ & 0.03 & Desogestrel & 0.15 \\
\hline & & & & 22 to 28 & - & - & - & - \\
\hline \multirow[t]{3}{*}{ Tri-Minulet } & \multirow[t]{3}{*}{$\begin{array}{l}\text { Combined oral } \\
\text { contraceptive }\end{array}$} & \multirow{3}{*}{$\begin{array}{l}\text { Milvane, Phaeva, Triadene, } \\
\text { Tricilomex, TriFemoden, } \\
\text { Trigynera, Tri-Gynera, } \\
\text { Trigynovin, Triodeen, } \\
\text { Trioden, Triodena, Triodene }\end{array}$} & \multirow[t]{3}{*}{ Triphasic } & 1 to 7 & $\begin{array}{l}\text { Ethinyl } \\
\text { estradiol }\end{array}$ & 0.03 & Gestodene & 0.05 \\
\hline & & & & 8 to 14 & $\begin{array}{l}\text { Ethinyl } \\
\text { estradiol }\end{array}$ & 0.04 & Gestodene & 0.07 \\
\hline & & & & 15 to 21 & $\begin{array}{l}\text { Ethinyl } \\
\text { estradiol }\end{array}$ & 0.03 & Gestodene & 0.1 \\
\hline \multirow[t]{2}{*}{ Seasonale } & \multirow[t]{2}{*}{$\begin{array}{l}\text { Combined oral } \\
\text { contraceptive }\end{array}$} & \multirow{2}{*}{$\begin{array}{l}\text { Quasense, Iclevia, Introvale, } \\
\text { Setlakin, Sylevia } \\
\text { Seasonique, Amethia is the } \\
\text { next generation of Seasonale } \\
\text { that contains } 0.01 \mathrm{mg} \text { E in } \\
\text { the inactive pills to suppress } \\
\text { breakthrough bleeding }\end{array}$} & \multirow[t]{2}{*}{$\begin{array}{l}\text { Extended } \\
\text { cycle }\end{array}$} & 1 to 84 & $\begin{array}{l}\text { Ethinyl } \\
\text { estradiol }\end{array}$ & 0.03 & Levonorgestrel & 0.15 \\
\hline & & & & 85 to 91 & - & - & - & - \\
\hline Ortho evra & $\begin{array}{l}\text { Combined } \\
\text { hormonal } \\
\text { contraceptive } \\
\text { (patch) }\end{array}$ & EVRA & Monophasic & $\begin{array}{l}\text { Every } 7 \\
\text { days }\end{array}$ & $\begin{array}{l}\text { Ethinyl } \\
\text { estradiol }\end{array}$ & 0.6 & Norelgestromin & 6 \\
\hline Mirena & $\begin{array}{l}\text { Progestin-only } \\
\text { (IUD) }\end{array}$ & Liletta, Levosert & & $\begin{array}{l}\text { Every } 5 \\
\text { years }\end{array}$ & - & - & Levonorgestrel & 52 \\
\hline \multirow{3}{*}{$\begin{array}{l}\text { IUD- } \\
\text { gestagen } \\
\text { releasing }\end{array}$} & \multirow{3}{*}{$\begin{array}{l}\text { Progestin-only } \\
\text { (IUD) }\end{array}$} & Progestasert & & & & & Progesterone & 38 \\
\hline & & Skyla, Jaydess & & $\begin{array}{l}\text { Every } 3 \\
\text { years }\end{array}$ & & & Levonorgestrel & 13.5 \\
\hline & & Kyleena & & $\begin{array}{l}\text { Every } 5 \\
\text { years }\end{array}$ & & & Levonorgestrel & 19.5 \\
\hline \multirow[t]{3}{*}{ Milvane } & \multirow[t]{3}{*}{$\begin{array}{l}\text { Combined oral } \\
\text { contraceptive }\end{array}$} & \multirow{3}{*}{$\begin{array}{l}\text { Phaeva, Triadene, } \\
\text { Tricilomex, TriFemoden, } \\
\text { Trigynera, Tri-Gynera, Tri- } \\
\text { Minulet, Trigynovin, } \\
\text { Triminulet, Triodeen, } \\
\text { Trioden, Triodena, } \\
\text { Triodene" }\end{array}$} & \multirow[t]{3}{*}{ Triphasic } & 1 to 7 & $\begin{array}{l}\text { Ethinyl } \\
\text { estradiol }\end{array}$ & 0.03 & Gestodene & 0.05 \\
\hline & & & & 7 to 14 & $\begin{array}{l}\text { Ethinyl } \\
\text { estradiol }\end{array}$ & 0.04 & Gestodene & 0.07 \\
\hline & & & & 15 to 21 & $\begin{array}{l}\text { Ethinyl } \\
\text { estradiol }\end{array}$ & 0.03 & Gestodene & 0.1 \\
\hline
\end{tabular}




\begin{tabular}{|c|c|c|c|c|c|c|c|c|}
\hline Microgynon & $\begin{array}{l}\text { Combined oral } \\
\text { contraceptive }\end{array}$ & & Monophasic & 1 to 21 & $\begin{array}{l}\text { Ethinyl } \\
\text { estradiol }\end{array}$ & 0.03 & Levonorgestrel & 0.15 \\
\hline \multirow[t]{4}{*}{ Ovcon } & \multirow[t]{4}{*}{$\begin{array}{l}\text { Combined oral } \\
\text { contraceptive }\end{array}$} & \multirow{4}{*}{$\begin{array}{l}\text { Ovcon 35, Ovcon 50, } \\
\text { Balziva, Briellyn, Femcon } \\
\text { Fe, Gildagia } \\
\text { Nexesta Fe, Philith, } \\
\text { Vyfemla, Wymzya Fe, } \\
\text { Zenchent } 0.4 / 35 \text {, Zenchent } \\
\text { Fe, Zeosa }\end{array}$} & \multirow[t]{4}{*}{ Monophasic } & 1 to 21 & $\begin{array}{l}\text { Ethinyl } \\
\text { estradiol }\end{array}$ & 0.035 & Norethindrone & 0.4 \\
\hline & & & & 22 to 28 & - & - & - & - \\
\hline & & & & 1 to 21 & $\begin{array}{l}\text { Ethinyl } \\
\text { estradiol }\end{array}$ & 0.05 & Norethindrone & 0.4 \\
\hline & & & & 22 to 28 & - & - & - & - \\
\hline \multirow[t]{2}{*}{ Minulet } & \multirow[t]{2}{*}{$\begin{array}{l}\text { Combined oral } \\
\text { contraceptive }\end{array}$} & \multirow[t]{2}{*}{ Sylvie 30, Femodene } & \multirow[t]{2}{*}{ Monophasic } & 1 to 21 & $\begin{array}{l}\text { Ethinyl } \\
\text { estradiol }\end{array}$ & 0.03 & Gestodene & 0.075 \\
\hline & & & & 22 to 28 & - & - & - & - \\
\hline \multirow[t]{2}{*}{ Novynette } & \multirow[t]{2}{*}{$\begin{array}{l}\text { Combined oral } \\
\text { contraceptive }\end{array}$} & \multirow[t]{2}{*}{$\begin{array}{l}\text { Desmin 20, Femina, } \\
\text { Mercilon }\end{array}$} & \multirow[t]{2}{*}{ Monophasic } & 1 to 21 & $\begin{array}{l}\text { Ethinyl } \\
\text { estradiol }\end{array}$ & 0.02 & Desogestrel & 0.15 \\
\hline & & & & 22 to 28 & - & - & - & - \\
\hline \multirow[t]{2}{*}{ Ovysmen } & \multirow[t]{2}{*}{$\begin{array}{l}\text { Combined oral } \\
\text { contraceptive }\end{array}$} & \multirow{2}{*}{$\begin{array}{l}\text { Ovysmen } 0.5 / 35, \text { Ovysmen } \\
1 / 35, \text { Brevicon } 0.5 / 35 \text {, Ortho } \\
0.5 / 35 \text {, Necon } 0.5 / 35, \\
\text { Nortrel } 0.5 / 35 \text {, Wera, } \\
\text { Modicon, Cyonanz } \\
\end{array}$} & \multirow[t]{2}{*}{ Monophasic } & 1 to 21 & $\begin{array}{l}\text { Ethinyl } \\
\text { estradiol }\end{array}$ & 0.035 & Norethindrone & 0.5 \\
\hline & & & & 1 to 21 & $\begin{array}{l}\text { Ethinyl } \\
\text { estradiol }\end{array}$ & 0.035 & Norethindrone & 1 \\
\hline Gestagen & - & - & - & - & - & - & - & - \\
\hline Ortho Evra & $\begin{array}{l}\text { Combined } \\
\text { hormonal } \\
\text { contraceptive } \\
\text { (patch) }\end{array}$ & EVRA & Monophasic & $\begin{array}{l}\text { Every } 7 \\
\text { days }\end{array}$ & $\begin{array}{l}\text { Ethinyl } \\
\text { estradiol }\end{array}$ & 0.6 & Norelgestromin & 6 \\
\hline $\begin{array}{l}\text { More than } \\
\text { one }\end{array}$ & - & - & - & - & - & - & - & - \\
\hline Camilla & $\begin{array}{l}\text { Progestin-only } \\
\text { (pill) }\end{array}$ & $\begin{array}{l}\text { Aygestin, Micronor, Errin, } \\
\text { Jolivette, Nora-BE, Nor-QD }\end{array}$ & Monophasic & Daily & - & - & Norethindrone & 0.35 \\
\hline Provera & $\begin{array}{l}\text { Not indicated } \\
\text { for } \\
\text { contraception }\end{array}$ & & & & - & - & $\begin{array}{l}\text { Medroxyprogesterone } \\
\text { acetate }\end{array}$ & $2.5>5>10$ \\
\hline Cerazette & $\begin{array}{l}\text { Progestin-only } \\
\text { (pill) }\end{array}$ & $\begin{array}{l}\text { Cerelle, Zelleta, Azalia, } \\
\text { Lactinette }\end{array}$ & Monophasic & Daily & - & - & Desogestrel & 0.075 \\
\hline Evra & $\begin{array}{l}\text { Combined } \\
\text { hormonal } \\
\text { contraceptive } \\
\text { (patch) }\end{array}$ & & Monophasic & $\begin{array}{l}\text { Every } 7 \\
\text { days }\end{array}$ & $\begin{array}{l}\text { Ethinyl } \\
\text { estradiol }\end{array}$ & 0.6 & Norelgestromin & 6 \\
\hline
\end{tabular}




\begin{tabular}{|c|c|c|c|c|c|c|c|c|}
\hline Rigevidon & $\begin{array}{l}\text { Combined oral } \\
\text { contraceptive }\end{array}$ & & Monophasic & 1 to 21 & $\begin{array}{l}\text { Ethinyl } \\
\text { estradiol }\end{array}$ & 0.03 & Levonorgestrel & 0.15 \\
\hline Nuvaring & $\begin{array}{l}\text { Combined } \\
\text { hormone } \\
\text { contraceptive } \\
\text { (ring) }\end{array}$ & & Monophasic & $\begin{array}{l}\text { Every } 21 \\
\text { days } / 28 \\
\text { days } / 35 \\
\text { days }\end{array}$ & $\begin{array}{l}\text { Ethinyl } \\
\text { estradiol }\end{array}$ & 2.6 & Etonogestrel & 11.4 \\
\hline Regulon & $\begin{array}{l}\text { Combined oral } \\
\text { contraceptive }\end{array}$ & Desorelle, Desmin 30 & Monophasic & 1 to 21 & $\begin{array}{l}\text { Ethinyl } \\
\text { estradiol }\end{array}$ & 0.03 & Desogestrel & 0.15 \\
\hline \multirow[t]{2}{*}{ Yasminelle } & \multirow[t]{2}{*}{$\begin{array}{l}\text { Combined oral } \\
\text { contraceptive }\end{array}$} & \multirow[t]{2}{*}{ Yaz, Beyaz, Gianvi } & \multirow[t]{2}{*}{ Monophasic } & 1 to 24 & \begin{tabular}{|l|}
$\begin{array}{l}\text { Ethinyl } \\
\text { estradiol }\end{array}$ \\
\end{tabular} & 0.02 & Drospirenone & 3 \\
\hline & & & & 25 to 28 & - & - & - & \\
\hline Duphaston & $\begin{array}{l}\text { Not indicated } \\
\text { for } \\
\text { contraception }\end{array}$ & & Monophasic & & - & - & Dydrogesterone & 10 \\
\hline \multirow[t]{2}{*}{ Femodene } & \multirow[t]{2}{*}{$\begin{array}{l}\text { Combined oral } \\
\text { contraceptive }\end{array}$} & \multirow[t]{2}{*}{ Sylvie 30, Minulet } & \multirow[t]{2}{*}{ Monophasic } & 1 to 21 & $\begin{array}{l}\text { Ethinyl } \\
\text { estradiol }\end{array}$ & 0.03 & Gestodene & 0.075 \\
\hline & & & & 22 to 28 & - & - & - & - \\
\hline Implanon & $\begin{array}{l}\text { Progestin-only } \\
\text { (implant) }\end{array}$ & Nexplanon & Monophasic & $\begin{array}{l}\text { Every } 3 \\
\text { years }\end{array}$ & - & - & Etonogestrel & 68 \\
\hline
\end{tabular}




\subsubsection{Exposure Classification}

The following variables were created for this descriptive analysis. Women were stratified into eight birth cohorts corresponding to each decade from 1920 to 2000: 1920-1929, 1930-1939, 1940-1949, 1950-1959, 1960-1969, 1970-1979, 1980-1989, 1990-1999. Specific contraceptive methods defined for this analysis include oral contraceptives, implants, injections, IUDs and other. Oral contraceptive users were defined as subjects who reported ever using an estrogenprogestin, progestin-only or nonspecific oral contraceptive pill. Implant users were defined as subjects who reported ever using Implanon or a nonspecific contraceptive implant. Injection users were defined as subjects who reported ever using Depo-Provera or a nonspecific injection. Type of IUD was classified as hormonal (LNG IUDs) or non-hormonal (metal-bearing or inert IUDs). IUD users were defined as subjects who reported ever using a hormonal or nonhormonal IUD. Subjects who indicated IUD use without reporting a specific IUD name were classified as nonhormonal IUD users if the year of initiation occurred prior to the year in which the hormonal IUD was introduced in each country. Other pertained to subjects who reported use of less popular contraceptive methods including vaginal ring and transdermal patch. For women who reported multiple methods of contraception, all methods were included.

\subsubsection{Statistical Analysis}

Descriptive statistics were used to summarize method-specific contraceptive use by birth cohort and mutation type through tabular and graphical means. Analyses were conducted for oral contraceptives, IUDs, implants, injections and other contraceptive methods. All analyses were performed using the SAS statistical package, version 9.1.3 (SAS Institute, Cary, NC). 


\subsection{Analysis of Contraceptive Use and BRCA Ovarian Cancer Risk}

Details of the study population, data collection and data storage methodology were previously described in Section 3.1.

\subsubsection{Exposure classification}

Any contraceptive use was defined as use of any method of hormonal contraception (including transdermal patches and vaginal rings) or a nonhormonal IUD. Specific contraceptive methods defined for this analysis include oral contraceptives, implants, injections and IUDs (defined in Section 3.1).

The following temporal characteristics of IUD use were created for this analysis. Among IUD ever users, the total duration of IUD use was calculated from the sum of all periods of IUD use prior to the censor date. Age at first IUD use was calculated by subtracting the date of birth from the date of first use. Age at last IUD use was calculated by subtracting the date of birth from the date of last use. Total duration of use, age at first use and age at last use were categorized into two groups: less than or equal to and greater than the median distribution. Recency of IUD use was categorized as never use, former use (discontinued more than six months prior) or current or recent use (discontinued within the last six months).

Contraceptive users who failed to indicate a medication name or method were classified as oral contraceptive users due to the high proportion of oral contraceptive use among BRCA mutation carriers in our cohort.

\subsubsection{Assessment of Cancer Outcomes}

The primary outcome of interest was a diagnosis of a primary invasive ovarian or fallopian tube cancer, collectively referred to as ovarian cancer henceforth. Diagnoses of ovarian cancer were identified by self-report. For each reported case of incident cancer in the follow-up questionnaire, pathology reports were requested to abstract information regarding histological subtype (serous, endometrioid, mucinous, clear cell), clinical staging (I-IV), primary site of 
origin (ovary, fallopian tube, peritoneum, other) and sites of spread (fallopian tubes, peritoneum, uterus, other). When pathology reports could not be obtained, medical records were requested to confirm the diagnosis. Pathology reports were not available for participants diagnosed prior to the baseline questionnaire.

\subsubsection{Study Subjects Available for Analysis}

A total of $17,893 B R C A 1$ or $B R C A 2$ mutation carriers were available for inclusion in this analysis. Subjects were excluded if they had a previous diagnosis of cancer other than breast or ovarian cancer $(\mathrm{n}=2,295)$, were missing information on personal history of breast or ovarian cancer $(\mathrm{n}=407)$, missing BRCA mutation type $(\mathrm{n}=197)$, had an oophorectomy prior to censor date $(n=77)$, missing information on oophorectomy status or year of surgery $(n=467)$, or missing date or birth or baseline date $(n=8)$. Subjects were also excluded if they were missing pertinent information regarding contraceptive use $(\mathrm{n}=237)$. After exclusions, a total of 14,205 $B R C A 1$ or $B R C A 2$ mutation carriers were available for analysis, including 2,765 women diagnosed with ovarian cancer (potential case subjects) and 15,128 women with no history of ovarian cancer (potential control subjects).

Case subjects were defined as women with a diagnosis of ovarian cancer in the baseline (prevalent) or follow-up period (incident). Control subjects were defined as women who never had ovarian cancer. A control was eligible to be matched to a case if the date of prophylactic oophorectomy in the control occurred at or after the year of ovarian cancer diagnosis of the matched case. Each case was matched to a single control subject according to year of birth (within one year), year of completion of the baseline questionnaire (within two years), country of residence (Canada, United States, Poland, Other), BRCA mutation type (BRCA1 or BRCA2) and personal history of breast cancer (yes/no). Matching variables were selected a priori as established confounders that influence risk of BRCA-ovarian cancer. In total, 1,732 matched pairs were generated. A flow diagram for the selection of study participants is provided in Figure 6. 
Excluded from analysis $(n=3,688)$

1. Diagnosis of cancer other than breast or ovarian cancer $(n=2,295)$

2. Missing personal history of breast or ovarian cancer $(n=407)$

3. Missing BRCA1/2 mutation type ( $\mathrm{n}=197)$

4. BSO prior to year of diagnosis in the case, or BSO prior to year of diagnosis of matched case in the control $(n=77)$

5. Missing information on oophorectomy status or year of surgery $(n=467)$

6. Missing information on birth control use $(n=237)$

7. No date of birth or baseline date $(n=8)$
Recruitment 1996-2021 ( $n=17,893)$

- Inclusion criteria: $B R C A 1 / 2$ mutation carrier, $\geq 18$ years old

4

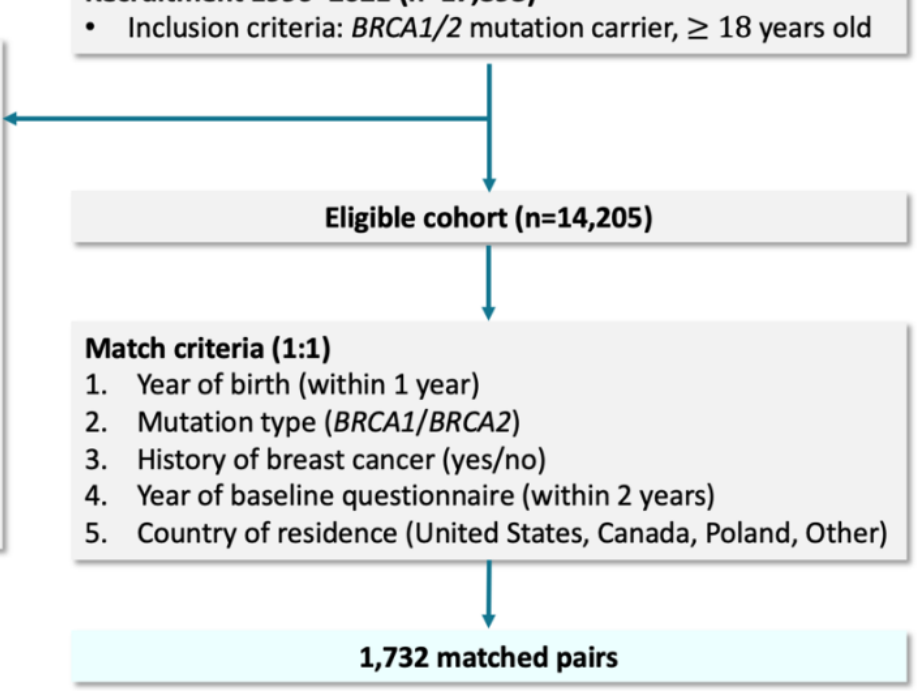

Figure 6. Recruitment statistics for a matched case-control study of contraceptive use and ovarian cancer risk among $B R C A$ mutation carriers 


\subsubsection{Statistical Analysis}

A matched case-control analysis was conducted to evaluate the associations between methods of contraceptive use and the risk of ovarian cancer. Distributions of continuous and categorical variables were compared between cases and controls using the Student's $t$-test and Chi-square test, respectively. Univariate and multivariate ORs and 95\% confidence intervals (CI) of ovarian cancer associated with each contraceptive exposure were estimated by conditional logistic regression. The multivariate model adjusted for the following covariates: parity (parous/nulliparous), history of breastfeeding (ever/never), menopausal status (premenopausal/postmenopausal) and previous oral contraceptive use (ever/never). Selection of covariates was based on the literature. Participants were categorized as postmenopausal if they had stopped menstruating for at least a year prior to censoring/event due to natural causes, radiation, or chemotherapy.

The follow-up period for this analysis is from the date of completion of the baseline questionnaire (i.e. study entry) until March $31^{\text {st }} 2021$. Cases were followed from study entry until the date of their ovarian cancer diagnosis, prophylactic oophorectomy, death or date of completion of their last follow-up questionnaire. For the controls, data on exposures was censored at the date of diagnosis of the matched case.

The analysis of any hormonal contraceptive included women who never used any hormonal contraception as the referent group. Each contraceptive method was then examined in a separate model with women who never used that respective method (not excluding other methods) before the censor date serving as the referent group. For select analyses with insufficient data, we used unconditional logistic regression to calculate age-adjusted and multivariate estimates (adjusted for aforementioned covariates as well as matching variables).

All analyses were performed using the SAS statistical package, version 9.1.3 (SAS Institute, Cary, NC). $P$-values were based on two-sided tests and were considered statistically significant if $P<0.05$. 


\section{Chapter 4. Results}

\subsection{Trends in Contraceptive use among BRCA Mutation Carriers}

\subsubsection{Subject Characteristics}

Demographic characteristics of the 2,254 women who reported a history of any contraceptive use are shown in Table 9. Of these women, $73 \%$ were BRCAl mutation carriers and $27 \%$ were BRCA2 mutation carriers. The mean year of birth was 1971 and 1966 for women with a BRCAI and BRCA2 mutation, respectively. There were low proportions of women in the earlier birth cohorts, from $0.04 \%$ in the $1920-1929$ birth cohort to $14 \%$ in the $1950-1959$ birth cohort. Only $3 \%$ of women belonged to the most recent birth cohort, which will likely increase as the cohort ages. The majority of data was derived from the United States, Canada and Poland. Oral contraceptives were the most popular contraceptive method, reported by $95 \%$ of $B R C A 1$ and BRCA2 mutation carriers born between 1920 and 1999 who had a history of any contraceptive use (Table 9). Lifetime rates of use for IUDs, implants and injections were lower at 9\%, 3\% and $4 \%$, respectively. Approximately $4 \%$ of women reported use of other hormonal contraceptive methods including the vaginal ring and transdermal patch at one point.

\subsubsection{Trends in Use of Various Contraceptive Methods Across Birth Cohorts}

The total number of contraceptive users rose from the 1920-1929 birth cohort and peaked at the 1970-1979 birth cohort (Table 10). The declining number of contraceptive users in more recent birth cohorts may be attributed to the older average age of our population. Overall, nearly all contraceptive users $(96 \%)$ reported a history of oral contraceptive use, which was consistent across birth cohorts (Table 10, Figure 7). Trends in the proportions of oral contraceptive use were broadly similar between $B R C A 1$ and $B R C A 2$ mutation carriers (Figure 8, Column totals may exceed $100 \%$ as women were allowed to report more than one method

Figure 9); however, only $88 \%$ of BRCA2 mutation carriers in the 1990-1999 birth cohort reported using oral contraceptives at some point in their lives, compared to $96 \%$ of $B R C A l$ mutation carriers (Table 11, 
Table 12). This difference is likely due to low numbers of contraceptive users with a BRCA2 mutation, which may increase as the cohort ages.

IUDs were the most commonly used LARC method. The highest proportions of lifetime IUD use were reported among women born in 1930-1939 (12\%) and 1990-1999 (12\%), although the 1930-1939 birth cohort was contained only eight contraceptive users (Table 10, Figure 7). Rates of IUD use remained fairly constant across birth cohorts, aside from a decline to around $6 \%$ in the 1960-1969 birth cohort for both BRCA1 and BRCA2 mutation carriers (Table 11,

Table 12). Regarding contraceptive injections, increasing proportions of successive cohorts reported lifetime injection use, $1 \%$ of those born in 1940-1949 to $6 \%$ of those born in 1970-1979 (Table 10, Column totals may exceed $100 \%$ as women were allowed to report more than one method

Figure 7). Implant use was initiated in the 1940-1949 cohort and rates remained stable for $B R C A 1$ and BRCA2 mutation carriers born after 1950. The greatest proportion of implant use was observed among BRCA mutation carriers born from 1970-1979 (4\%). For BRCA2 mutation carriers, 12\% uptake of both implants and injections were observed for the 1990-1999 birth cohort, but likely attributed to low numbers of total contraceptive users (Table 12). Taken together, lifetime rates of LARC use increased across birth cohorts from 12\% in the 1930-1939 cohort to $22 \%$ in the $1970-1979$ cohort. Use of other hormonal contraceptive methods increased steadily from less than $1 \%$ of the 1950-1959 cohort to approximately $9 \%$ of the 1980-1989 birth cohort, which did not vary by BRCA mutation type (Table 10, Column totals may exceed $100 \%$ as women were allowed to report more than one method

Figure 7). Rates of injections and other hormonal contraceptive methods were slightly lower among the youngest birth cohort (1990-1999), but this cohort has yet to reach menopause and remain within the period of initiation of use. 
Table 9. Demographic characteristics of contraceptive users with a $B R C A 1$ or $B R C A 2$ mutation

\begin{tabular}{|c|c|c|c|}
\hline \multirow[t]{2}{*}{ Characteristic } & \multirow[t]{2}{*}{ Total $(n=2,254)$} & \multicolumn{2}{|l|}{ Mutation } \\
\hline & & $B R C A 1(\mathrm{n}=1,652)$ & $B R C A 2(\mathrm{n}=602)$ \\
\hline Mean year of birth & 1970.12 & 1971.49 & 1966.34 \\
\hline $\begin{array}{r}\text { Birth cohort, } \mathrm{n}(\%) \\
1920-1929 \\
1930-1939 \\
1940-1949 \\
1950-1959 \\
1960-1969 \\
1970-1979 \\
1980-1989 \\
1990-1999\end{array}$ & $\begin{array}{l}1(0.04) \\
8(0.35) \\
113(5.01) \\
325(14.42) \\
564(25.02) \\
687(30.48) \\
498(22.09) \\
58(2.57)\end{array}$ & $\begin{array}{l}0(0.00) \\
4(0.18) \\
59(3.57) \\
203(12.29) \\
396(23.97) \\
533(32.3) \\
407(24.6) \\
50(3.0)\end{array}$ & $\begin{array}{l}1(0.2) \\
4(0.7) \\
54(9.0) \\
122(20.3) \\
168(27.9) \\
154(25.6) \\
91(15.1) \\
8(1.3)\end{array}$ \\
\hline $\begin{array}{l}\text { Contraceptive methods, } \mathrm{n}(\%) \\
\text { Oral contraceptive } \\
\text { Intrauterine device } \\
\text { Injection } \\
\text { Implant } \\
\text { Other }\end{array}$ & $\begin{array}{l}2,142(95.03) \\
210(9.32) \\
95(4.21) \\
68(3.02) \\
102(4.53)\end{array}$ & $\begin{array}{l}1,559(94.37) \\
145(8.78) \\
65(3.93) \\
58(3.51) \\
82(4.96)\end{array}$ & $\begin{array}{l}583(96.84) \\
65(10.80) \\
30(4.98) \\
10(1.66) \\
10(3.32)\end{array}$ \\
\hline $\begin{array}{l}\text { Country of residence, } \mathrm{n}(\%) \\
\text { United States } \\
\text { Canada } \\
\text { Poland } \\
\text { Other }\end{array}$ & $\begin{array}{l}536(23.80) \\
726(32.20) \\
785(34.80) \\
207(9.20)\end{array}$ & $\begin{array}{l}311(18.83) \\
395(23.91) \\
775(46.91) \\
171(10.35)\end{array}$ & $\begin{array}{l}225(37.38) \\
331(54.98) \\
10(1.66) \\
36(5.98) \\
\end{array}$ \\
\hline
\end{tabular}


Table 10. Distribution of $B R C A 1$ and $B R C A 2$ mutation carriers who reported ever use of various contraceptive methods by birth cohort

\begin{tabular}{|c|c|c|c|c|c|c|c|c|}
\hline \multirow{2}{*}{ Contraceptive method } & \multicolumn{8}{|c|}{ Birth cohort } \\
\hline & $1920-1929$ & 1930-1939 & 1940-1949 & 1950-1959 & 1960-1969 & $1970-1979$ & 1980-1989 & 1990-1999 \\
\hline Oral contraceptive, $\mathrm{n}(\%)$ & $1(100.00)$ & $8(100.00)$ & $110(97.35)$ & $307(94.46)$ & $535(94.86)$ & $649(94.47)$ & $477(95.78)$ & $55(94.83)$ \\
\hline Intrauterine device, $\mathrm{n}(\%)$ & $0(0.00)$ & $1(12.50)$ & $10(8.85)$ & $30(9.23)$ & $31(5.50)$ & $80(11.64)$ & $51(10.24)$ & $7(12.07)$ \\
\hline Injection, $\mathrm{n}(\%)$ & $0(0.00)$ & $0(0.00)$ & $1(0.88)$ & $7(2.15)$ & $23(4.08)$ & $41(5.97)$ & $20(4.02)$ & $2(3.45)$ \\
\hline Implant, n (\%) & $0(0.00)$ & $0(0.00)$ & $1(0.88)$ & $10(3.08)$ & $14(2.48)$ & $28(4.08)$ & $13(2.61)$ & $2(3.45)$ \\
\hline Other, n (\%) & $0(0.00)$ & $0(0.00)$ & $0(0.00)$ & $2(0.62)$ & $7(1.24)$ & $43(6.26)$ & $46(9.24)$ & $4(6.90)$ \\
\hline Total, n (\%) & $1(100.00)$ & $8(100.00)$ & $113(100.00)$ & $325(100.00)$ & $564(100.00)$ & $687(100.00)$ & $498(100.00)$ & $58(100.00)$ \\
\hline
\end{tabular}

Column totals may exceed $100 \%$ as women were allowed to report more than one method

Figure 7. Trends in proportions of $B R C A 1$ and $B R C A 2$ mutation carriers who reported ever use of various contraceptive methods by birth cohort

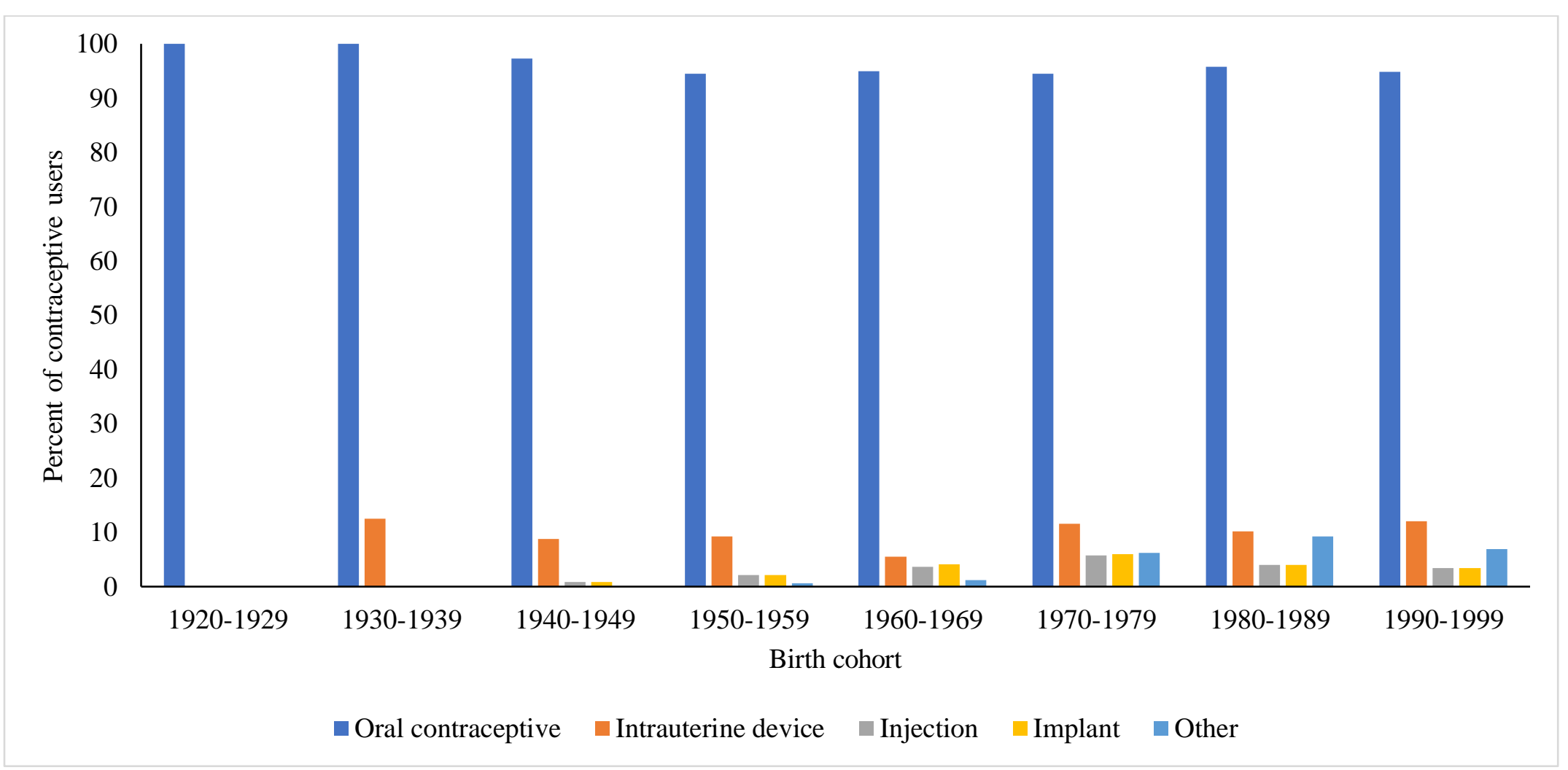


Table 11. Distribution of $B R C A 1$ mutation carriers who reported ever use of various contraceptive methods by birth cohort

\begin{tabular}{|c|c|c|c|c|c|c|c|c|}
\hline \multirow{2}{*}{ Contraceptive method } & \multicolumn{8}{|c|}{ Birth cohort } \\
\hline & 1920-1929 & 1930-1939 & $1940-1949$ & 1950-1959 & 1960-1969 & 1970-1979 & 1980-1989 & 1990-1999 \\
\hline Oral contraceptive, n (\%) & $0(0.00)$ & $4(100.00)$ & $57(96.60)$ & $188(92.6)$ & $370(93.4)$ & $503(94.4)$ & $389(95.6)$ & $48(96.0)$ \\
\hline Intrauterine device, n (\%) & $0(0.00)$ & $0(0.00)$ & $5(8.47)$ & $20(9.85)$ & $21(5.30)$ & $60(11.26)$ & $36(8.85)$ & $3(6.00)$ \\
\hline Injection, n (\%) & $0(0.00)$ & $0(0.00)$ & $0(0.00)$ & $5(2.46)$ & $16(4.04)$ & $28(5.25)$ & $15(3.69)$ & $1(2.00)$ \\
\hline Implant, n (\%) & $0(0.00)$ & $0(0.00)$ & $1(1.69)$ & $10(4.93)$ & $13(3.28)$ & $23(4.32)$ & $10(2.46)$ & $1(2.00)$ \\
\hline Other, n (\%) & $0(0.00)$ & $0(0.00)$ & $0(0.00)$ & $2(0.99)$ & $6(1.52)$ & $34(6.38)$ & $36(8.85)$ & $4(8.00)$ \\
\hline Total, n (\%) & $0(100.00)$ & $4(100.00)$ & $59(100.00)$ & $203(100.00)$ & $396(100.00)$ & $533(100.00)$ & $407(100.00)$ & $50(100.00)$ \\
\hline
\end{tabular}

Column totals may exceed $100 \%$ as women were allowed to report more than one method

Figure 8. Trends in proportions of BRCA1 mutation carriers who reported ever use of various contraceptive methods by birth cohort

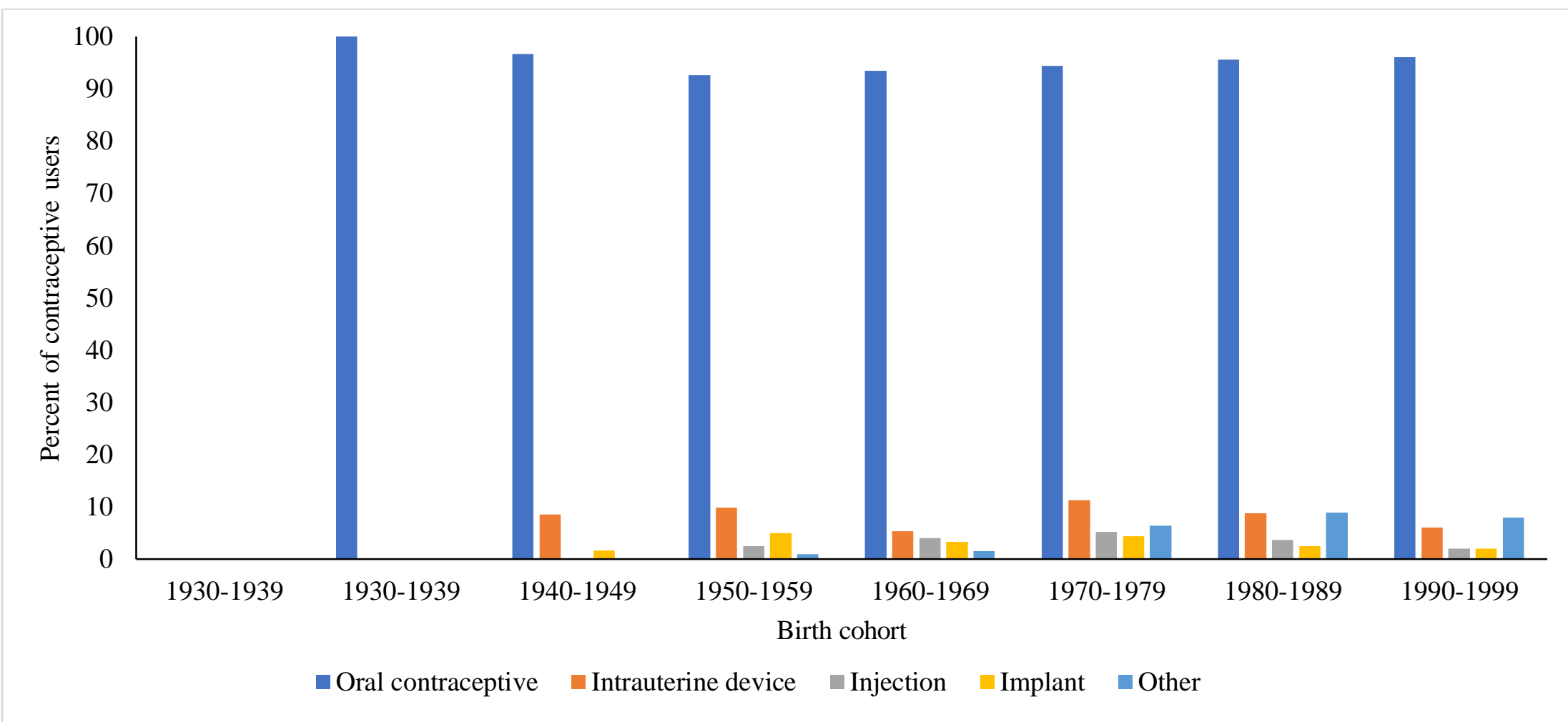


Table 12. Distribution of $B R C A 2$ mutation carriers who reported ever use of various contraceptive methods by birth cohort

\begin{tabular}{|c|c|c|c|c|c|c|c|c|}
\hline Contraceptive method & \multicolumn{7}{|c|}{ Birth cohort } \\
\cline { 2 - 9 } & $1920-1929$ & $1930-1939$ & $1940-1949$ & $1950-1959$ & $1960-1969$ & $1970-1979$ & $1980-1989$ & $1990-1999$ \\
\hline Oral contraceptive, $\mathrm{n}(\%)$ & $1(100.0)$ & $4(100.00)$ & $53(98.15)$ & $119(97.54)$ & $165(98.21)$ & $146(94.81)$ & $88(96.70)$ & $7(87.50)$ \\
\hline Intrauterine device, $\mathrm{n}(\%)$ & $0(0.00)$ & $1(25.00)$ & $5(9.26)$ & $10(8.20)$ & $10(5.95)$ & $20(12.99)$ & $15(16.48)$ & $4(50.0)$ \\
\hline Injection, $\mathrm{n}(\%)$ & $0(0.00)$ & $0(0.00)$ & $1(1.85)$ & $2(1.64)$ & $8(4.76)$ & $13(8.44)$ & $5(5.49)$ & $1(12.50)$ \\
\hline Implant, $\mathrm{n}(\%)$ & $0(0.00)$ & $0(0.00)$ & $0(0.00)$ & $0(0.00)$ & $1(0.60)$ & $5(3.25)$ & $3(3.30)$ & $1(12.50)$ \\
\hline Other, $\mathrm{n}(\%)$ & $0(0.00)$ & $0(0.00)$ & $0(0.00)$ & $0(0.00)$ & $1(0.60)$ & $9(5.84)$ & $10(10.99)$ & $0(0.00)$ \\
\hline Total, $\mathrm{n}(\%)$ & $1(100.00)$ & $4(100.00)$ & $54(100.00)$ & $122(100.00)$ & $168(100.00)$ & $154(100.00)$ & $91(100.00)$ & $8(100.00)$ \\
\hline
\end{tabular}

Column totals may exceed $100 \%$ as women were allowed to report more than one method

Figure 9. Trends in proportions of $B R C A 2$ mutation carriers who reported ever use of various contraceptive methods by birth cohort

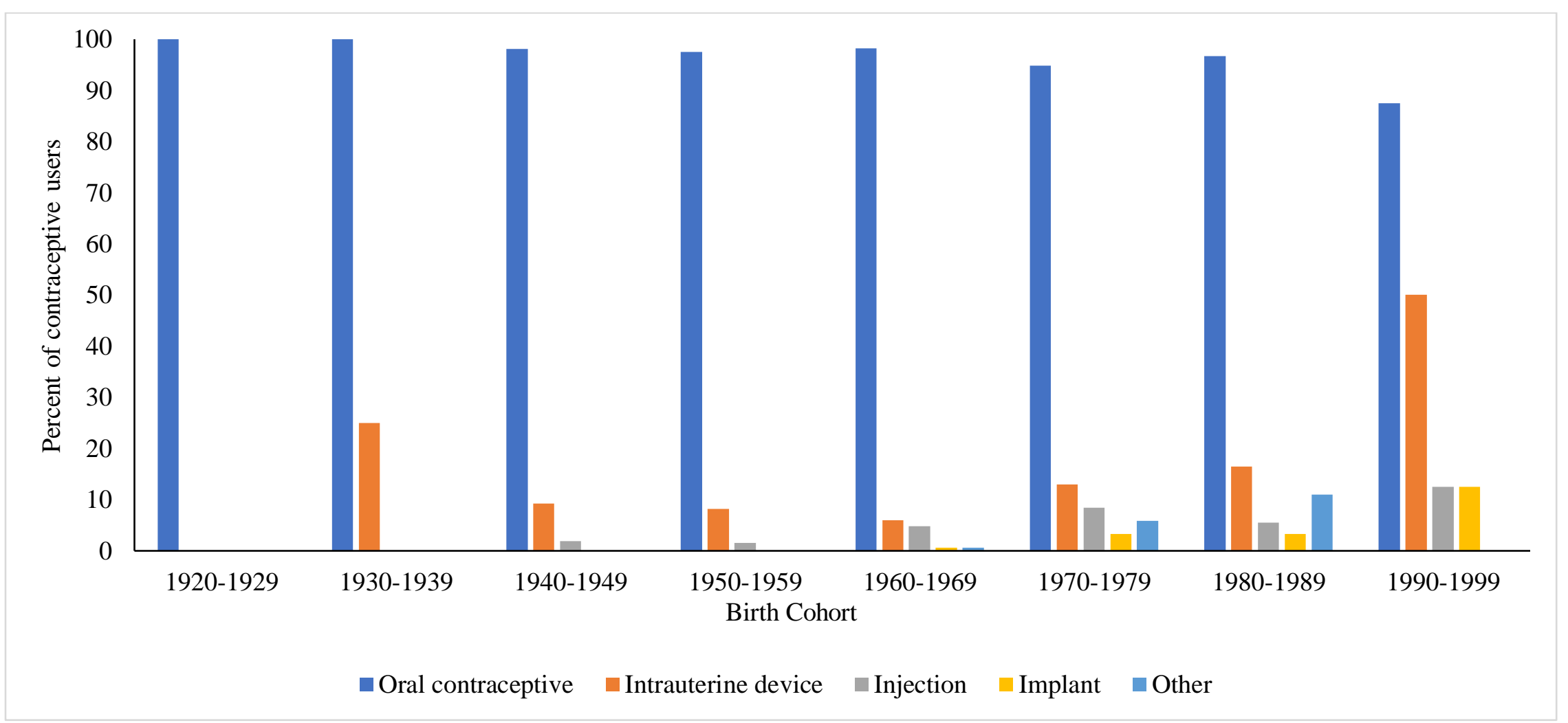




\subsection{Associations between Contraceptive Method and BRCA Ovarian Cancer Risk}

\subsubsection{Subject Characteristics}

Table 13 describes the demographic, reproductive, hormonal and other clinical characteristics of the 1,732 cases of ovarian cancer and 1,732 matched controls that were included in the final analysis. Cases and controls did not differ with respect to year of birth, country of residence, $B R C A$ gene mutation type, and personal history of breast cancer. On average, BRCAl mutation carriers were diagnosed with ovarian cancer at a younger age than BRCA2 mutation carriers (48.94 years vs. 53.60 years). Fewer cases than controls reported a history of breastfeeding (63.14\% vs. $67.68 \% ; P=0.008)$. Mean parity and mean months of breastfeeding were slightly lower in cases than controls (2.69 vs. $2.82, P=0.008$ for parity; 13.19 months vs. 14.76 months, $P=0.02$ for breastfeeding). Cases were more likely to have a history of HRT use (20.64\% vs. $13.45 \% ; P<0.0001)$.

Table 14 shows the characteristics of women who reported ever use of each contraceptive method. Oral contraceptives were the most popular contraceptive method adopted by $49.22 \%$ of the cohort. The mean duration of oral contraceptive use was 7.41 years (range 0.08-32 years). The mean age at first use was 24 years (range 6-53 years) and the mean age at last use was 31 years (range 6-61 years). Overall, only 56 women reported use of an IUD (1.62\%). Of those, the nonhormonal IUD was the most common method $(n=35 ; 62 \%)$ with only 13 women reporting use of a hormonal IUD (23\%). The mean duration of IUD use was 6.85 years (range 0.08-24 years). On average, women reported a longer duration of use for the nonhormonal IUD (8.0 years; range 1-24 years) than the hormonal IUD (4.85 years; 0.08-19 years). The mean age at first use of any IUD was 30.53 years (range 19-50 years) and the mean age at last use of any IUD was 36.98 years (range 20-52 years). Women who used a hormonal IUD reported a later average age at first use (34.15 years; range 20-48 years) and last use (39 years; range 26-49 years) than women who used a nonhormonal IUD (28.50 years, range 19-42 years for age at first use; 35.85 years, range 20-52 years for age at last use). There were 25 women who had an implant $(0.72 \%)$ for a mean duration of 6.67 years (range 1-20 years). The mean age at initiation was 29.12 years (range 20-42 years) and the mean age at last use was 35.73 years (range 22-48 years). Injections 
were the least common contraceptive method reported by $0.23 \%$ of the cohort. Among injection users, the average age at first use is 29.25 years (range 22-37 years) over a mean duration of 3.77 years (range 0.42-19 years). The mean age at last use of an injection was 33.75 (range 24-44 years). On average, women who used oral contraceptives were older than women who used other methods (mean year of birth, 1953).

\subsubsection{Associations between Methods of Contraceptive Use and Ovarian Cancer Risk}

The associations between various methods of contraceptive use and the risk of developing ovarian cancer are presented in Table 15. Cases were less likely to have a history of oral contraceptive use $(45.09 \%$ vs. $53.35 \% ; P<0.0001)$ and implant use $(0.35 \%$ vs. $1.10 \% ; P<0.001)$ than controls. Compared to never users of any hormonal contraceptive, women who used any type of hormonal contraceptive had a significant $38 \%$ reduction in risk of developing ovarian cancer according to the univariate model $(\mathrm{OR}=0.62 ; 95 \% \mathrm{CI} 0.53-0.73)$. The relationship was similar after adjusting for previous oral contraceptive use, history of breastfeeding, menopausal status and parity $(\mathrm{OR}=0.58 ; 95 \%$ CI $0.48-0.70)$.

Oral contraceptive use was associated with a significant $41 \%$ reduction in the risk of ovarian cancer $(\mathrm{OR}=0.59 ; 95 \% \mathrm{CI}$ 0.49-0.71). Use of contraceptive implants and injections were associated with a $62 \%$ to $68 \%$ reduction in risk respectively; however, analyses were based on a small number of women and should be interpreted with caution $(\mathrm{OR}=0.38,95 \% \mathrm{CI}$ 0.15-0.97 for implants; OR=0.32, 95\% CI 0.063-1.66 for injections). Only six cases and 19 controls reported ever use of an implant. Only two cases and six controls reported ever use of an injection, and results did not achieve statistical significance (Table 15).

Women who used an IUD had a nonsignificant decreased risk of ovarian cancer compared to women who never used an IUD (OR=0.81; 95\% CI 0.46-1.45). Although limited by a small number of users, the OR for hormonal IUDs was 0.91 (95\% CI 0.28-2.91) and the OR for nonhormonal IUDs was 0.66 (95\% CI 0.31-1.39) (Table 15). Temporal characteristics of IUD use including duration of use, recency of use, age at first use and age at last use were not significantly associated with risk of ovarian cancer $(P \geq 0.15)$ (Table 16). 
Although based on a small number of exposed cases, the associations did not vary substantially by menopausal status (Table 15). In the analysis stratified by history of oral contraceptive use, implant use was associated with a greatly decreased risk of ovarian cancer among women who never used contraceptives $(\mathrm{OR}=0.23 ; 95 \% \mathrm{CI} 0.01-0.80)$ (Table 17). 
Table 13. Comparison of ovarian cancer cases and controls with a BRCA1 or BRCA2 mutation

\begin{tabular}{|c|c|c|c|}
\hline Characteristic & $\begin{array}{l}\text { Cases } \\
(n=1,732)\end{array}$ & $\begin{array}{l}\text { Controls } \\
(\mathrm{n}=1,732)\end{array}$ & $P$ \\
\hline Year of birth, mean (range) & $1952.50(1917-1986)$ & $1952.60(1917-1987)$ & Matched \\
\hline $\begin{array}{c}\text { Age at diagnosis, mean (range) } \\
\text { BRCA1 } \\
B R C A 2\end{array}$ & $\begin{array}{l}48.94(17-84) \\
53.60(22-82)\end{array}$ & $\begin{array}{l}\mathrm{n} / \mathrm{a} \\
\mathrm{n} / \mathrm{a}\end{array}$ & $\mathrm{n} / \mathrm{a}$ \\
\hline $\begin{array}{c}\text { Country of residence, } \mathrm{n}(\%) \\
\text { United States } \\
\text { Canada } \\
\text { Poland } \\
\text { Other } \\
\end{array}$ & $\begin{array}{l}466(26.91) \\
382(22.06) \\
645(37.24) \\
239(13.80) \\
\end{array}$ & $\begin{array}{l}466(26.91) \\
382(22.06) \\
645(37.24) \\
239(13.80) \\
\end{array}$ & Matched \\
\hline $\begin{array}{c}\text { Mutation type, } \mathrm{n}(\%) \\
\text { BRCA1 } \\
\text { BRCA2 }\end{array}$ & $\begin{array}{l}1,443(83.31) \\
289(16.69)\end{array}$ & $\begin{array}{l}1,443(83.31) \\
289(16.69)\end{array}$ & Matched \\
\hline $\begin{array}{l}\text { Personal history of breast cancer, } \mathrm{n}(\%) \\
\text { No } \\
\text { Yes }\end{array}$ & $\begin{array}{l}1,131(65.30) \\
601(34.70) \\
\end{array}$ & $\begin{array}{l}1,131(65.30) \\
601(34.70) \\
\end{array}$ & Matched \\
\hline $\begin{array}{c}\text { Parity, } \mathrm{n}(\%) \\
\text { Nulliparous } \\
\text { Parous } \\
\text { Missing }\end{array}$ & $\begin{array}{l}264(15.30) \\
1,462(84.70) \\
6\end{array}$ & $\begin{array}{l}225(13.00) \\
1,506(87.00) \\
1\end{array}$ & 0.05 \\
\hline Number of pregnancies ${ }^{\mathrm{a}}$, mean (range) & $2.69(1-8)$ & $2.82(1-10)$ & 0.008 \\
\hline $\begin{array}{l}\text { Breastfeeding history, } \mathrm{n}(\%) \\
\text { Never } \\
\text { Ever } \\
\text { Missing } \\
\text { Months of breastfeeding, mean (range) }\end{array}$ & $\begin{array}{l}656(36.86) \\
942(63.14) \\
180 \\
13.19(0.04-137)\end{array}$ & $\begin{array}{l}491(32.32) \\
1028(67.68) \\
213 \\
14.76(0.10-159)\end{array}$ & $\begin{array}{l}0.008 \\
0.02\end{array}$ \\
\hline Age at menarche (year), mean (SD) & $13.11(4-28)$ & $13.17(8-18)$ & 0.24 \\
\hline Age at menopause (year), mean (SD) & $46.66(23-59)$ & $46.50(26-68)$ & 0.52 \\
\hline $\begin{array}{l}\text { Menopausal status } \mathrm{b}^{\mathrm{b}}, \mathrm{n}(\%) \\
\text { Premenopausal } \\
\text { Postmenopausal } \\
\text { Missing }\end{array}$ & $\begin{array}{l}855(51.35) \\
810(48.65) \\
67\end{array}$ & $\begin{array}{l}905(54.03) \\
770(45.97) \\
57\end{array}$ & 0.12 \\
\hline $\begin{array}{l}\text { HRT use, } \mathrm{n}(\%) \\
\text { Never } \\
\text { Ever } \\
\text { Missing } \\
\end{array}$ & $\begin{array}{l}1373(79.36) \\
357(20.64) \\
2\end{array}$ & $\begin{array}{l}1493(86.55) \\
232(13.45) \\
7\end{array}$ & $<0.0001$ \\
\hline $\begin{array}{c}\text { Tubal ligation, } \mathrm{n}(\%) \\
\text { Never } \\
\text { Ever } \\
\text { Missing } \\
\end{array}$ & $\begin{array}{l}1393(86.68) \\
214(13.32) \\
125\end{array}$ & $\begin{array}{l}1314(84.60) \\
239(15.39) \\
179\end{array}$ & 0.10 \\
\hline $\begin{array}{l}\text { Hysterectomy, n (\%) } \\
\text { Never } \\
\text { Ever } \\
\text { Missing } \\
\end{array}$ & $\begin{array}{l}1508(92.97) \\
114(7.03) \\
110\end{array}$ & $\begin{array}{l}1416(92.19) \\
120(7.81) \\
196\end{array}$ & 0.40 \\
\hline $\begin{array}{l}\text { Oral contraceptive use, } \mathrm{n}(\%) \\
\text { Never } \\
\text { Ever }\end{array}$ & $\begin{array}{l}951(54.91) \\
781(45.09)\end{array}$ & $\begin{array}{l}808(46.65) \\
924(53.35)\end{array}$ & $<0.0001$ \\
\hline $\begin{array}{c}\text { Implant use, } \mathrm{n}(\%) \\
\text { Never } \\
\text { Ever }\end{array}$ & $\begin{array}{l}1,726(99.65) \\
6(0.35)\end{array}$ & $\begin{array}{l}1,713(98.90) \\
19(1.10)\end{array}$ & 0.009 \\
\hline $\begin{array}{c}\text { Injection use, } \mathrm{n}(\%) \\
\text { Never }\end{array}$ & $1,730(99.88)$ & $1,726(99.65)$ & 0.16 \\
\hline
\end{tabular}




\begin{tabular}{|c|l|l|l|}
\hline Ever & $2(0.12)$ & $6(0.35)$ & \\
\hline IUD use, $\mathrm{n}(\%)$ & & & 0.42 \\
Never & $1,707(98.56)$ & $1,701(98.21)$ & \\
Ever & $25(1.44)$ & $31(1.79)$ & \\
\hline
\end{tabular}

Abbreviations: SD, standard deviation; HRT, hormone replacement therapy; IUD, intrauterine device

${ }^{\mathrm{a} A m o n g}$ parous

${ }^{\mathrm{b}}$ Control subjects with age at menopause less than their age at censor date were classified as postmenopausal. Case subjects with age at menopause less than their age at diagnosis were classified as postmenopausal. Case subjects with age of menopause equivalent to their age at diagnosis due to cancer-related surgery were classified as premenopausal. 
Table 14. Characteristics of users of various contraceptive methods with a BRCA1 or BRCA2 mutation

\begin{tabular}{|c|c|c|c|c|c|c|}
\hline Characteristic & $\begin{array}{l}\text { Any IUD } \\
(n=56)\end{array}$ & $\begin{array}{l}\text { Hormonal IUD } \\
(\mathrm{n}=13,23 \%)^{\mathrm{a}}\end{array}$ & $\begin{array}{l}\text { Nonhormonal } \\
\text { IUD }(n=35)^{\mathrm{a}}\end{array}$ & $\begin{array}{l}\text { Implant } \\
(\mathrm{n}=25)\end{array}$ & $\begin{array}{l}\text { Injection } \\
(\mathrm{n}=8)\end{array}$ & $\begin{array}{l}\text { Oral contraceptive } \\
(n=1,705)\end{array}$ \\
\hline Year of birth, mean (range) & $\begin{array}{l}1957(1941- \\
1983)\end{array}$ & $\begin{array}{l}1967(1956- \\
1983)\end{array}$ & 1953 (1941-1972) & $1955(1945-1972)$ & $1962(1955-1973)$ & $1953(1920-1987)$ \\
\hline $\begin{array}{c}\text { Recency of use, } \mathrm{n}(\%) \\
\text { Current or recent } \\
\text { Former }\end{array}$ & $\begin{array}{l}14(25) \\
42(75)\end{array}$ & $\begin{array}{l}9(69.23) \\
4(30.77)\end{array}$ & $\begin{array}{l}4(11.43) \\
31(88.57)\end{array}$ & $\begin{array}{l}2(8) \\
23(92)\end{array}$ & $\begin{array}{l}0(0) \\
8(100)\end{array}$ & $\begin{array}{l}51(10.30) \\
444(89.70)\end{array}$ \\
\hline Years of use, mean (range) & $6.85(0.08-24)^{\mathrm{b}}$ & $4.85(0.08-19)$ & $8.00(1-24)^{\mathrm{e}}$ & $6.67(1-20)^{\mathrm{h}}$ & $3.77(0.42-19)^{\mathrm{i}}$ & $7.41(0.08-32)^{j}$ \\
\hline Age at last use, mean (range) & $36.98(20-52)^{\mathrm{c}}$ & $39.00(26-49)$ & $35.85(20-52)^{\mathrm{g}}$ & $35.75(22-48)^{\mathrm{h}}$ & $33.75(24-44)$ & $31.04(6-61)^{1}$ \\
\hline Age at first use, mean (range) & $30.53(19-50)^{\mathrm{d}}$ & $34.15(20-48)$ & $28.50(19-42)^{\mathrm{f}}$ & $29.12(20-42)^{\mathrm{h}}$ & $29.25(22-37)$ & $23.60(6-53)^{\mathrm{k}}$ \\
\hline
\end{tabular}

Abbreviations: IUD, intrauterine device.

aUD users who reported use of both hormonal and nonhormonal IUDs $(n=1)$. IUD users who were missing IUD type (n=9).

${ }^{b}$ IUD users excluded due to missing start year or stop year $(n=4)$.

'IUD users excluded due to missing stop year $(n=3)$.

dIUD users excluded due to missing start year $(n=3)$.

e'IUD users excluded due to missing start year or stop year $(n=2)$.

${ }^{f}$ IUD users excluded due to missing start year $(n=1)$.

gIUD users excluded due to missing stop year $(n=1)$.

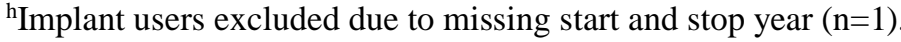

${ }^{\mathrm{i}}$ Injection users excluded due to missing duration of use $(n=1)$.

Oral contraceptive users excluded due to missing duration of use $(n=23)$.

${ }^{\mathrm{k}}$ Oral contraceptive users excluded due to missing start and stop year $(\mathrm{n}=16)$.

${ }^{j}$ Oral contraceptive users excluded due to missing stop and/or stop year $(n=19)$ 
Table 15. Associations between use of various contraceptive methods and ovarian cancer risk among women with $B R C A 1$ or $B R C A 2$ mutation, stratified by menopausal status.

\begin{tabular}{|c|c|c|c|c|c|}
\hline Variable & Cases/controls & $\begin{array}{l}\text { Univariate OR } \\
(95 \% \text { CI })\end{array}$ & $P$ & $\begin{array}{l}\text { Multivariate OR } \\
(95 \% \text { CI) }\end{array}$ & $P$ \\
\hline \multicolumn{6}{|l|}{ Overall } \\
\hline \multicolumn{6}{|l|}{ Any contraceptive } \\
\hline Never $^{\mathrm{a}}$ & $931 / 785$ & & & Ref & \\
\hline Ever & $801 / 947$ & $0.62(0.53-0.73)$ & $<0.0001$ & $0.58(0.48-0.70)^{\mathrm{b}}$ & $<0.0001$ \\
\hline \multicolumn{6}{|l|}{ Oral contraceptive } \\
\hline Never & $951 / 808$ & & & 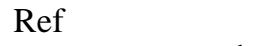 & \\
\hline \multicolumn{6}{|l|}{ Implant } \\
\hline Never & $1726 / 1713$ & & & Ref & \\
\hline Ever & $6 / 19$ & $0.32(0.13-0.79)$ & 0.01 & $0.38(0.15-0.97)^{\mathrm{c}}$ & 0.04 \\
\hline \multicolumn{6}{|l|}{ Injection } \\
\hline Never & $1730 / 1726$ & & & & \\
\hline Ever & & $0.33(0.07-1.65)$ & 0.18 & $0.32(0.06-1.66)^{\mathrm{c}}$ & 0.18 \\
\hline \multicolumn{6}{|l|}{ IUD use } \\
\hline $\begin{array}{l}\text { Never } \\
\text { Ever }\end{array}$ & $\begin{array}{l}1 / 0 / / 1 / 01 \\
25 / 31\end{array}$ & $\begin{array}{l}\text { Ref } \\
0.81(0.48-1.37)\end{array}$ & 0.42 & $0.81(0.46-1.45)^{\mathrm{c}}$ & 0.48 \\
\hline \multicolumn{6}{|l|}{ Type of IUD } \\
\hline Never & $1707 / 1701$ & & & & \\
\hline Hormonal & $6 / 7$ & $0.86(0.29-2.55)$ & 0.78 & $0.91(0.28-2.91)^{\mathrm{c}}$ & 0.87 \\
\hline Non-hormonal & $14 / 21$ & $0.67(0.34-1.31)$ & 0.24 & $0.66(0.31-1.39)^{\mathrm{c}}$ & 0.27 \\
\hline \multicolumn{6}{|l|}{ Premenopausal } \\
\hline \multicolumn{6}{|l|}{ Any contraceptive } \\
\hline Never $^{\mathrm{a}}$ & $423 / 368$ & Ref & & Ref & \\
\hline Ever & $432 / 532$ & $0.63(0.49-0.81)$ & 0.0002 & $0.57(0.43-0.75)^{\mathrm{b}}$ & $<0.0001$ \\
\hline \multicolumn{6}{|l|}{ Oral contraceptive } \\
\hline Never & $432 / 380$ & & & & \\
\hline Ever & $423 / 520$ & $0.63(0.49-0.81)$ & 0.0003 & $0.57(0.43-0.75)^{\mathrm{b}}$ & $<0.0001$ \\
\hline \multicolumn{6}{|l|}{ Implant } \\
\hline Ever & & $0.43(0.11-1.66)$ & 0.22 & $0.60(0.15-2.37)^{\mathrm{c}}$ & 0.47 \\
\hline \multicolumn{6}{|l|}{ Injection } \\
\hline Never & $854 / 895$ & Ref & & Ref & \\
\hline Ever & & $0.25(0.03-2.24)$ & 0.22 & $0.24(0.03-2.18)^{\mathrm{c}}$ & 0.20 \\
\hline \multicolumn{6}{|l|}{ IUD use } \\
\hline $\begin{array}{l}\text { Never } \\
\text { Ever }\end{array}$ & $\begin{array}{l}840 / 890 \\
15 / 10\end{array}$ & $\begin{array}{l}\text { Ret } \\
1.50(0.67-3.34)\end{array}$ & 0.32 & $1.53(0.65-3.60)^{\mathrm{c}}$ & 0.33 \\
\hline \multicolumn{6}{|l|}{$\underline{\text { Postmenopausal }}$} \\
\hline \multicolumn{6}{|l|}{ Any contraceptive } \\
\hline Never $^{\mathrm{a}}$ & $508 / 417$ & & & & \\
\hline Ever & $369 / 415$ & $0.63(0.48-0.83)$ & 0.0010 & $0.57(0.42-0.79)^{\mathrm{b}}$ & 0.0005 \\
\hline Oral contraceptive & & & & & \\
\hline Never & $519 / 428$ & Ref & & Ref & \\
\hline Ever & $358 / 404$ & $0.62(0.47-0.81)$ & 0.0006 & $0.57(0.41-0.79)^{\mathrm{b}}$ & 0.0007 \\
\hline Never & $875 / 822$ & Ref & & Ref & \\
\hline Ever & $2 / 10$ & $0.33(0.07-1.65)$ & 0.18 & $0.44(0.09-2.24)^{\mathrm{c}}$ & 0.33 \\
\hline
\end{tabular}




\begin{tabular}{|c|l|l|l|l|l|}
\hline Injection & & & & \\
$\begin{array}{c}\text { Never } \\
\text { Ever }\end{array}$ & $876 / 831$ & Ref & & Ref & \\
IUD use & & $0.84(0.05-13.50)^{\mathrm{d}}$ & 0.90 & $0.89(0.05-15.30)^{\mathrm{e}}$ & 0.94 \\
$\begin{array}{l}\text { Never } \\
\text { Ever }\end{array}$ & $867 / 811$ & Ref & & Ref & \\
\hline
\end{tabular}

Abbreviations: IUD, intrauterine device; CI, confidence interval.

${ }^{\text {a}}$ Referent group includes women who never reported use of any hormonal contraceptive.

${ }^{\mathrm{b}}$ Adjusted for history of breastfeeding (ever/never), menopausal status (premenopausal/ postmenopausal), parity (ever/never).

${ }^{\mathrm{c}}$ Adjusted for previous oral contraceptive use (ever/never), history of breastfeeding (ever/never), menopausal status (premenopausal/postmenopausal), parity (ever/never).

${ }^{\mathrm{d}}$ Analyzed by unconditional logistic regression due to insufficient data. Adjusted for age (continuous)

${ }^{\mathrm{e}}$ Analyzed by unconditional logistic regression due to insufficient data. Adjusted for history of breastfeeding (ever/never), menopausal status (premenopausal/postmenopausal), parity (ever/never), age (continuous), year of baseline questionnaire (continuous), country of residence (United States, Canada, Poland, Other), history of breast cancer (ever/never), BRCA mutation type (BRCA1/BRCA2) 
Table 16. Temporal associations between IUD use and ovarian cancer risk among $B R C A 1$ or $B R C A 2$ mutation carriers

\begin{tabular}{|c|c|c|c|c|c|}
\hline Variable & Cases/controls & $\begin{array}{l}\text { Univariate OR } \\
(95 \% \mathrm{CI})\end{array}$ & $P$ & $\begin{array}{l}\text { Multivariate OR } \\
(95 \% \text { CI })^{\mathrm{a}}\end{array}$ & $P$ \\
\hline \multicolumn{6}{|l|}{ Duration of IUD use ${ }^{b}$} \\
\hline Never $^{\mathrm{c}}$ & $1707 / 1701$ & & & & \\
\hline$<5$ years & $9 / 13$ & $0.69(0.30-1.62)$ & 0.40 & $0.75(0.31-1.82)$ & 0.52 \\
\hline$\geq 5$ years & $15 / 15$ & $1.00(0.49-2.05)$ & 1.00 & $0.91(0.41-2.03)$ & 0.82 \\
\hline p-trend & & $0.98(0.92-1.04)$ & 0.44 & $0.97(0.91-1.04)$ & 0.41 \\
\hline \multicolumn{6}{|l|}{ Recency of IUD use } \\
\hline Never & $1707 / 1701$ & Ref & & Ref & \\
\hline Current o & $4 / 10$ & $0.40(0.12-1.28)$ & 0.12 & $0.38(0.10-1.41)$ & 0.15 \\
\hline Former & $21 / 21$ & $1.00(0.55-1.83)$ & 1.00 & $1.00(0.53-1.97)$ & 0.96 \\
\hline \multicolumn{6}{|l|}{ Age at first IUD use ${ }^{\mathrm{d}}$} \\
\hline Never & $1707 / 1701$ & Ref & & Ref & \\
\hline$\leq 29$ ye & $14 / 14$ & $1.00(0$ & 1.00 & 0.89 & 0.77 \\
\hline$>29$ years & $10 / 15$ & $0.67(0.30-1.48)$ & 0.32 & $0.73(0.30-1.76)$ & 0.48 \\
\hline \multicolumn{6}{|l|}{ Age at last IUD use } \\
\hline Never & $1707 / 1701$ & & & Ref & \\
\hline$<37$ years & $14 / 15$ & $0.93(0.45-1.93)$ & 0.85 & $0.92(0.42-1.98)$ & 0.82 \\
\hline$\geq 37$ years & $11 / 16$ & $0.69(0.32-1.48)$ & 0.34 & $0.70(0.29-1.67)$ & 0.42 \\
\hline
\end{tabular}

Abbreviations: IUD, intrauterine device; CI, confidence interval.

${ }^{a}$ Adjusted for previous oral contraceptive use (ever/never), history of breastfeeding (ever/never), menopausal status (premenopausal/postmenopausal), parity (ever/never).

${ }^{b}$ IUD users excluded due to missing start year or stop year $(n=4)$.

${ }^{c}$ Referent group includes women who never reported use of any IUD.

dIUD users excluded due to missing start year $(\mathrm{n}=3)$.

'IUD users excluded due to missing stop year $(\mathrm{n}=3)$. 
Table 17. Associations between use of various LARC methods and ovarian cancer risk among women with $B R C A 1$ or $B R C A 2$ mutation, stratified by history of oral contraceptive use

\begin{tabular}{|c|c|c|c|c|c|}
\hline Variable & Cases/controls & $\begin{array}{l}\text { Univariate OR } \\
(95 \% \text { CI) }\end{array}$ & $P$ & $\begin{array}{l}\text { Multivariate } \text { OR }^{\mathrm{a}} \\
(\mathbf{9 5 \%} \mathrm{CI})\end{array}$ & $P$ \\
\hline \multicolumn{6}{|c|}{ No history of oral contraceptive use } \\
\hline \multicolumn{6}{|l|}{ Implant } \\
\hline Never & $950 / 798$ & 1.00 & Ref & 1.00 & Ref \\
\hline Ever & $1 / 10$ & $0.11(0.01-0.88)$ & 0.04 & $0.10(0.01-0.80)$ & 0.03 \\
\hline \multicolumn{6}{|l|}{ Injection } \\
\hline Never & $950 / 805$ & 1.00 & Ref & 1.00 & Ref \\
\hline \multirow{2}{*}{\multicolumn{6}{|c|}{ IUD use }} \\
\hline & & & & & \\
\hline Never & $946 / 800$ & 1.00 & Ref & 1.00 & Ref \\
\hline Ever & $5 / 8$ & $0.71(0.23-2.25)$ & 0.57 & $0.42(0.11-1.61)$ & 0.20 \\
\hline \multicolumn{6}{|c|}{ History of oral contraceptive use } \\
\hline \multicolumn{6}{|l|}{ Implant } \\
\hline Never & $776 / 915$ & 1.00 & Ref & 1.00 & Ref \\
\hline Ever & $5 / 9$ & $0.66(0.22-1.96)^{\mathrm{b}}$ & 0.45 & $0.60(0.20-1.83)^{\mathrm{c}}$ & 0.37 \\
\hline \multicolumn{6}{|l|}{ Injection } \\
\hline Never & $780 / 921$ & 1.00 & & 1.00 & Ref \\
\hline Ever & $1 / 3$ & $0.39(0.04-3.80)^{\mathrm{b}}$ & 0.42 & $0.44(0.05-4.31)^{\mathrm{c}}$ & 0.48 \\
\hline \multicolumn{6}{|l|}{ IUD use } \\
\hline Never & $761 / 901$ & 1.00 & Ref & 1.00 & Ref \\
\hline Ever & $20 / 23$ & $1.56(0.67-3.59)$ & 0.30 & $1.43(0.59-3.45)$ & 0.42 \\
\hline
\end{tabular}

Abbreviations: IUD, intrauterine device; CI, confidence interval.

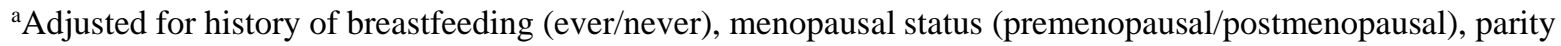
(ever/never)

${ }^{\mathrm{b}}$ Analyzed by unconditional logistic regression due to insufficient data. Adjusted for age (continuous)

${ }^{\mathrm{c}}$ Analyzed by unconditional logistic regression due to insufficient data. Adjusted for history of breastfeeding (ever/never), menopausal status (premenopausal/postmenopausal), parity (ever/never), age (continuous), year of baseline questionnaire (continuous), country of residence (United States, Canada, Poland, Other), history of breast cancer (ever/never), BRCA mutation type (BRCAl/BRCA2) 


\section{Chapter 5. Discussion}

\subsection{General Discussion}

Trends in contraceptive use across birth cohorts for BRCAl mutation carriers largely parallels that of $B R C A 2$ mutation carriers, despite fluctuations among the fewer women with a $B R C A 2$ mutation. The lifetime rates of oral contraceptive use did not vary between birth cohorts and remained high among all women born after 1920; however, use of LARCs, including implants, IUDs and injections increased successively across birth cohorts. To our knowledge, this is the first study to investigate trends in method-specific contraceptive use among BRCA mutation carriers by birth cohort.

These patterns are consistent with findings from nationally representative cross-sectional studies of the United States, Canada and Europe that report significant increases in the use of LARC methods over time. From 2006 to 2017, the annual increase in IUD and implant use was 6.2\% and $12.4 \%$ respectively among premenopausal women in the United States, while oral contraceptive use decreased by $2 \%$ annually ${ }^{245}$. During the same period, rates of IUD use increased significantly from $4.3 \%$ to $8.8 \%$ among Canadian women aged 15 to $50^{246}$. Data from the National Health Service indicate a similar trend of increasing LARC uptake from $26 \%$ in 2009 to $46 \%$ in 2019, while oral contraceptives declined from $45 \%$ in 2014 to $38 \%$ in $2019^{247}$.

Of note, the low prevalence of lifetime IUD use in the 1960-1969 birth cohort corresponds to a rapid decline in the popularity of IUDs in the 1990s. By 1995, IUDs were used by less than $1 \%$ of the United States population due to controversies of the Dalkon Shield and related complications of pelvic inflammatory disease and infertility ${ }^{248}$. The introduction of hormonal IUDs in North America by the early 2000s prompted a resurgence of IUD use in the ensuing decades. In an analysis of the 2012 National Surveys of Family Growth data, Mosher et al. reported that the highest proportion of IUD users were aged 25 to $34^{249}$. This corresponds to the 1970-1979 and 1980-1989 birth cohorts that saw an increase in rates of IUD use in our study.

Implants and injections played a minor role in the overall use of hormonal contraception in our cohort. Nonetheless, results suggest that the highest proportion of injection and implant use 
occurred in the 1970-1979 birth cohort, which aligns with the timing of approval of these methods in Canada and the United States ${ }^{165,250}$. Women born between 1970 and 1979 would be of reproductive age during the 1990s and ideal candidates for the introduction of these modes of contraception.

As the landscape of contraceptive usage changes, it is of interest to evaluate the relationships between various types of contraception and ovarian cancer risk in women with $B R C A$ mutations who possess increased predisposition for developing ovarian cancer. Oral contraceptives represent an established protective factor among high-risk women, but the potential benefit of other contraceptive methods is not clear. As such, the goal of the present study was to investigate the associations between different modes of contraception and the risk of developing ovarian cancer among women with an inherited BRCA1 or BRCA2 mutation

Results from our case-control study of 1,732 finely matched pairs suggest a significantly reduced risk of ovarian cancer with use of any contraceptive method, which was driven by the significant inverse association with use of implants or oral contraceptives. There was suggestion of a reduced risk with use of contraceptive injections and IUDs, although this was based on a small number of exposed cases and confidence intervals were wide. To our knowledge, this represents the first report of the association between contemporary contraceptive methods and the risk of ovarian cancer in high-risk women.

We showed an inverse but nonsignificant association with use of any type of IUD compared to never use among carriers. Consistent with our results, a recent meta-analysis of nine casecontrol and two cohort studies observed an inverse association between ever use of an IUD and ovarian cancer risk in the general population $(\mathrm{OR}=0.68 \text {; 95\% CI } 0.62-0.75)^{229}$. We further examined risk of ovarian cancer by types of IUD and detailed patterns of IUD use. Although not statistically significant, we observed cancer-protective effects with use of both hormonal and nonhormonal IUD types. Several population-based cohort studies have corroborated these findings, demonstrating significant decreases in risk of ovarian cancer for users of LNG IUDs ${ }^{231,232}$. These studies were combined in a meta-analysis by Balayla et al. that reported a standardized incidence ratio of 0.58 (95\% CI 0.47-0.71) for use of the LNG IUD specifically ${ }^{230}$. 
As rates of hormonal IUD use now surpass that of nonhormonal devices, future epidemiologic studies are needed to confirm the consistency and magnitude of risk reductions associated with this IUD type ${ }^{245}$.

With respect to nonhormonal IUDs, a recent pooled analysis of data from the New England Case-Control Study and Nurses' Health Studies reported no association between IUD use and ovarian cancer risk, wherein the majority of IUD use was attributed to nonhormonal methods ${ }^{233}$. Conversely, findings from the Shanghai Women's Health Study indicated that long-term IUD use ( $\geq 20$ years) was associated with reduced ovarian cancer risk in China, where the high prevalence of IUD use was dominated by the stainless steel ring ${ }^{227}$. Although the average duration of nonhormonal IUD use in our cohort was only eight years, our results agree with that of the latter report and suggest a nonsignificant decrease in risk of ovarian cancer with use of the nonhormonal IUD in BRCA mutation carriers.

Interestingly, Tworoger and colleagues observed a significantly elevated risk of ovarian cancer with IUD use in the Nurses' Health Study, which was attributed to possible IUD-related inflammation in the peritoneal cavity $(\mathrm{RR}=1.76 \text {; } 95 \% \mathrm{CI} 1.08-2.85)^{234}$. Both hormonal and nonhormonal IUDs elicit a foreign body reaction, a local sterile inflammatory response characterized by the influx of immune cells to the intrauterine environment ${ }^{251}$. The cellular and humoral components of the foreign body reaction extend to the extrauterine space and represent a mechanism by which IUDs promote the elimination of premalignant cells at the ovary and fallopian tube ${ }^{204}$. Nonetheless, inflammatory factors are also associated with ovarian carcinogenesis by generating genotoxic stress ${ }^{70}$. One hypothesis to reconcile these contradicting theories considers the timing of IUD use ${ }^{252}$. IUDs stimulate an inflammatory milieu that may be more protective for older women, who are more likely to have accumulated a greater number of precancerous cells than younger women. This hypothesis is consistent with our finding of an inverse (albeit nonsignificant) trend with increasing age at last IUD use.

Few studies in the general population have assessed the relationship between duration of IUD use and ovarian cancer risk to mixed results. Huang and colleagues found that long-term IUD use of at least 20 years was associated with a $38 \%$ reduction in risk $(\mathrm{HR}=0.62 ; 95 \% \mathrm{CI} 0.40$ - 
$0.97)^{228}$. Ness and colleagues observed the opposite relationship, such that a shorter duration of IUD use ( $\leq 4$ years) was associated with reduced risk of ovarian cancer $(\mathrm{OR}=0.53$; 95\% CI 0.390.72 ) and longer duration of IUD use ( $\geq 10$ years) trended toward increased risk (OR=1.40; $95 \%$ CI $0.82-2.39)^{253}$. In the present study, protection against ovarian cancer was not associated with duration of use in high-risk women, but only 52 IUD users informed the analysis.

Our finding of a $62 \%$ to $68 \%$ reduction in ovarian cancer risk with use of contraceptive implants or injections was based on a total of eight exposed cases; however, these results are consistent with the one other study that estimated risk of ovarian cancer among implant users in the general population $^{227}$. The authors reported a nonsignificant inverse association between implant use and ovarian cancer risk compared to never users ( $\mathrm{RR}=0.51 ; 95 \% \mathrm{CI} 0.07-3.64)$. Any protective effect may have been underestimated because history of contraceptive use prior to study entry was not collected. Regarding contraceptive injections, a recent pooled analysis of seven casecontrol studies in the Ovarian Cancer Association Consortium concluded a reduced risk of ovarian cancer with use of DMPA injections, which is in line with the majority of other retrospective data $224-226$. The few cohort studies that explored injection use and ovarian cancer risk have showed inconsistent results. In the Shanghai Women's Health Study of 70,259 Chinese women and 174 cases, injectables were not associated with ovarian cancer risk ${ }^{228}$. On the other hand, the Danish Sex Register Hormone Study observed increased risk with DMPA shots in a subgroup analysis of exclusive use until first switch $\left(R R=6.56 ; 95 \%\right.$ CI 6.56-20.40) ${ }^{227}$. Both studies were limited by a low prevalence of injection users and imprecise risk estimates.

Oral contraceptive use is an established protective factor for ovarian cancer in the general and carrier population alike $116,210,213,254$. In the current study, we found that oral contraceptive use was associated with a $41 \%$ decreased risk of developing ovarian cancer among BRCA mutation carriers. This result is in agreement with our two earlier reports on this topic, which were based on a subset of carriers also included in the current investigation ${ }^{79,244}$. Findings from the most recent analysis indicated a similar level of risk reduction with oral contraceptive use for $B R C A 1$ mutation carriers $(\mathrm{OR}=0.60 ; 95 \% \mathrm{CI} 0.50-0.71)$ and $B R C A 2$ mutation carriers $(\mathrm{OR}=0.63 ; 95 \%$ CI $0.39-0.80)^{79}$. Compared to our previous publications, the results here include an additional 403 cases and represent the most updated analysis of oral contraceptive use and ovarian cancer 
risk among women with a $B R C A$ mutation. The protective effects of oral contraceptives have been observed across all tumour histologic subtypes, but there is some evidence to suggest that the associations between oral contraceptive use may be stronger for serous and clear cell ovarian cancers ${ }^{120,255,256}$. Although we were not able to stratify by histotype, BRCA-associated ovarian cancers tend to be of endometrioid or high-grade serous subtype ${ }^{14}$.

Several mechanisms may explain the inverse relationships between various contraceptive methods and ovarian cancer risk. According to the 'incessant ovulation' hypothesis, the proliferative behaviour of the ovarian surface epithelium and the acute inflammatory environment at the distal fallopian tube following ovulation may predispose cells to DNA damage and malignant transformation ${ }^{62,65}$. Under this paradigm, ovulation inhibition via suppression of pituitary gonadotropins may be the primary biological mechanism by which oral contraceptives and injections impart protection against the development of ovarian cancer; however, the protective effects of these methods varied by magnitude, which implies additional mechanisms at play.

Alternate theories highlight a potential protective effect of high progestogen exposure ${ }^{82}$. In experimental studies, progestogens have exhibited pro-apoptotic and antiproliferative effects on transformed cells of the ovary as well as a necroptotic effect on abnormal cells of the fallopian tube epithelium ${ }^{92,93,257,258}$. Likewise, epidemiologic data reveal protective associations with pregnancy and breastfeeding, physiologic states characterized by elevated levels of progesterone ${ }^{79}$. Use of oral contraceptives, implants, IUDs and injections that contain highly potent synthetic progestins reinforce this theory by increasing exogenous progestogenic exposure compared to levels experienced by regularly cycling women ${ }^{259}$. This mechanism may be especially relevant among Mirena IUD users, for whom LNG has been measured at significant levels in the peritoneal fluid ${ }^{260}$. Further, the $68 \%$ risk reduction observed with injection use in our population is consistent with the high serum concentrations of DMPA attained, five-fold that of equivalent oral contraceptive use ${ }^{224}$. On the other hand, the majority of implant users in our cohort likely used LNG implants that do not consistently inhibit ovulation and sustain low serum concentrations of LNG, $25 \%$ to $50 \%$ that of oral contraceptives ${ }^{138}$. Although LNG is a highly 
potent progestin, the mechanism by which implants confer significant protection against ovarian cancer requires further investigation.

Lastly, inhibition of retrograde menstruation may explain the protective effect observed with hormonal IUD use. Hormonal IUDs are associated with sparse menstruations or amenorrhea in over $20 \%$ of users ${ }^{261}$. This is thought to reduce retrograde flow of menstrual blood, thereby minimizing local inflammation in the peritoneal cavity and malignant transformation at the site of the ovarian surface epithelium and distal fallopian tube ${ }^{72}$. Retrograde transport of endometrial cells can further implant as endometriosis, which has been linked to ovarian cancers of endometrioid, clear cell and low-grade serous histology ${ }^{77}$. Although data on tumour histology was not available in the present study, approximately $12 \%$ of $B R C A$-associated ovarian cancers have an endometrioid histotype ${ }^{14}$. Other studies in the general population have shown that women who use a LNG IUD have a lower risk of ovarian carcinomas of endometrial origin, supporting a partial preventive effect through of inhibition of menstruation ${ }^{79,232}$. 


\subsection{Strengths and Limitations}

This represents the first study to investigate the effects of different contraceptive methods on ovarian cancer risk in women with $B R C A 1$ or $B R C A 2$ mutation. Although only very small proportions of women used methods other than oral contraceptives, our analysis was strengthened by the large number of mutation carriers and the strict matching criteria to account for differences between cases and controls. We also obtained information about contraceptive use at baseline and biennially through follow-up questionnaires. Therefore, we were able to account for changes in exposure status, including method switches, cease of use or initiation of use during the follow-up period.

We also acknowledge important limitations to this research. First, given the small number of women who used implants, injections and IUDs, use of progestin-only contraceptives generated imprecise estimates and associations must be interpreted with caution. As a result, we had limited power to examine associations by BRCA mutation type, ovarian cancer histologic subtype or site of origin. Nonetheless, there has not been significant variability by histology in prior analyses of injectables or IUDs, and previous studies of oral contraceptive use among highrisk women have reported similar protective effects in carriers of either mutation type $79,124,224,232,239$. Secondly, there were too few women who reported use of a transdermal patch or vaginal ring to analyze these methods separately. When these women were excluded, the results did not change substantially. Thirdly, we were not sufficiently powered in our analysis stratified by history of oral contraceptive use and additional studies are necessary to confirm if the other modes of contraceptives independently impact risk. Fourth, it is important to note that the results of this study apply exclusively to premenopausal hormone use. The LNG IUD is additionally indicated for endometrial protection in HRT regimens. Further study is necessary to determine if postmenopausal IUD use provides ovarian cancer risk reduction for carriers who undergo natural menopause. Furthermore, hormonal IUDs were first launched in Finland in 1990 and only approved in North America in the early $2000 \mathrm{~s}^{180}$. More follow-up time may be required to capture changes in the direction or magnitude of association, considering the long latency period of ovarian cancer. Exposure misclassification may have affected our results because this analysis was based in self-reported contraceptive data. Despite this, previous studies have demonstrated fairly accurate recall of contraceptive history obtained through self- 
administered questionnaires ${ }^{262}$. Survivorship bias may influence our findings because prevalent cases comprised $87 \%$ of ovarian cancer cases included in our study, with an average of four years elapsed between the time of diagnosis to enrollment. Therefore, our results may be less representative for women with aggressive cases of ovarian cancer who died before enrolment. Nonetheless, the characteristics of the prevalent cases were similar to that of the incident cases (data not shown). Lastly, although there was a significant difference in the proportion of cases and controls who used hormone replacement therapy ( $21 \%$ vs. $13 \% ; P<0.0001)$, we have previously demonstrated that hormone replacement therapy does not influence the risk of ovarian cancer in this cohort ${ }^{127}$. 


\subsection{Future Directions and Conclusion}

Concerns have been raised regarding the progestin content of hormonal contraception as a possible risk factor for breast cancer. We previously reported an increased risk of breast cancer with oral contraceptive use at an early age in BRCAl mutation carriers ${ }^{263}$. Moreover, we found a possible increased risk of breast cancer with estrogen-progesterone hormone replacement therapy among women with a BRCA1 or BRCA2 mutation, but not for estrogen therapy alone ${ }^{264}$. This is in line with an emerging role for the progesterone-mediated receptor activator of nuclear factor КB (RANK) signalling pathway in BRCAl breast carcinogenesis ${ }^{265-267}$. Shamseddin et al. used ex vivo and xenograft models to demonstrate that androgenic progestins including LNG stimulate proliferation of breast epithelial cells via upregulation of the RANK ligand pathway ${ }^{268}$. These findings are also consistent with the epidemiologic association between hormonal IUD use and breast cancer risk. A recent meta-analysis of five case-control studies and two cohort studies concluded that women who use the LNG IUD have a $16 \%$ increased risk of breast cancer $(\mathrm{OR}=1.16 ; 95 \% \mathrm{CI} 1.06-1.28)^{269}$. Given growing uptake of the hormonal IUD, the role of IUD use on the risk of $B R C A 1$-associated breast cancer would be an important avenue of investigation.

For premenopausal women electing for permanent contraception, it is important to acknowledge surgical sterilization methods with potential ovarian cancer-protective effects. Tubal ligation has been associated with lower risk of developing ovarian cancer in previous studies of high-risk women; however, no relationship was observed in the most recent cohort study of 5,038 BRCA mutation carriers $(\mathrm{HR}=1.09 ; 95 \% \mathrm{CI} 0.83-1.45)^{73,76,244,270,271}$. Bilateral salpingectomy with delayed oophorectomy is also the subject of ongoing clinical trials to determine the effect on ovarian cancer incidence. Although beyond the scope of this project, tubal ligation and salpingectomy are gaining attention as highly effective contraceptive methods with potential roles in ovarian cancer prevention for high-risk women.

In conclusion, our findings confirm the well-established protective role of oral contraceptive use on the risk of developing ovarian cancer specifically among women with a BRCA1 or BRCA2 mutation. We also demonstrated a decreased risk with implant use and a potentially protective role of IUDs and injections. In agreement with our previous recommendations, oral 
contraceptives should be discussed as an effective contraceptive option and a likely cancerprotective factor during the risk assessment and counseling of this high risk population ${ }^{79}$. At this time, injections, implants and IUDs should not be contraindicated for BRCA mutation carriers who are considering these highly effective methods of contraception, although whether the level of risk reduction is equivalent to combined oral contraceptives warrants additional study. As non-oral, LARC methods continue to gain popularity, studies with larger numbers of users will further clarify the influence of these contraceptive methods on ovarian cancer development. It will be of interest to evaluate the differential impact of contraceptives on risk of cancers originating in the ovaries versus the fallopian tubes and whether contraceptives may improve survival outcomes for $B R C A$ mutation carriers. 


\section{Chapter 6. References}

1. Sung H, Ferlay J, Siegel RL, et al. Global Cancer Statistics 2020: GLOBOCAN Estimates of Incidence and Mortality Worldwide for 36 Cancers in 185 Countries. CA A Cancer J Clin. 2021;71(3):209-249. doi:10.3322/caac.21660

2. Xian W, George S. Meeting report from the 2018 12th Biennial Ovarian Cancer Research Symposium detection and prevention of ovarian cancer. Int J Gynecol Cancer. 2019;29(Suppl 2):s2-s6. doi:10.1136/ijgc-2019-000454

3. Malvezzi M, Carioli G, Rodriguez T, Negri E, La Vecchia C. Global trends and predictions in ovarian cancer mortality. Annals of Oncology. 2016;27(11):2017-2025.

doi:10.1093/annonc/mdw306

4. Sopik V, Iqbal J, Rosen B, Narod SA. Why have ovarian cancer mortality rates declined? Part I. Incidence. Gynecologic Oncology. 2015;138(3):741-749. doi:10.1016/j.ygyno.2015.06.017

5. Walsh T, Casadei S, Lee MK, et al. Mutations in 12 genes for inherited ovarian, fallopian tube, and peritoneal carcinoma identified by massively parallel sequencing. Proceedings of the National Academy of Sciences. 2011;108(44):18032-18037. doi:10.1073/pnas.1115052108

6. Singer CF, Tan YY, Muhr D, et al. Association between family history, mutation locations, and prevalence of BRCA1 or 2 mutations in ovarian cancer patients. Cancer Med. 2019;8(4):1875-1881. doi:10.1002/cam4.2000

7. Norquist BM, Harrell MI, Brady MF, et al. Inherited Mutations in Women With Ovarian Carcinoma. JAMA Oncol. 2016;2(4):482. doi:10.1001/jamaoncol.2015.5495

8. Zhang S, Royer R, Li S, et al. Frequencies of BRCA1 and BRCA2 mutations among 1,342 unselected patients with invasive ovarian cancer. Gynecologic Oncology. 2011;121(2):353357. doi:10.1016/j.ygyno.2011.01.020

9. Gorodetska I, Kozeretska I, Dubrovska A. BRCA Genes: The Role in Genome Stability, Cancer Stemness and Therapy Resistance. J Cancer. 2019;10(9):2109-2127. doi: $10.7150 /$ jca. 30410

10. Petrucelli N, Daly MB, Feldman GL. Hereditary breast and ovarian cancer due to mutations in BRCA1 and BRCA2. Genet Med. 2010;12(5):245-259.

doi:10.1097/GIM.0b013e3181d38f2f

11. Kuchenbaecker KB, Hopper JL, Barnes DR, et al. Risks of Breast, Ovarian, and Contralateral Breast Cancer for BRCA1 and BRCA2 Mutation Carriers. JAMA. 2017;317(23):2402. doi:10.1001/jama.2017.7112 
12. Kotsopoulos J, Gronwald J, Karlan B, et al. Age-specific ovarian cancer risks among women with a BRCA1 or BRCA2 mutation. Gynecologic Oncology. 2018;150(1):85-91. doi:10.1016/j.ygyno.2018.05.011

13. Mersch J, Jackson MA, Park M, et al. Cancers associated with BRCA 1 and BRCA 2 mutations other than breast and ovarian: BRCA and Other Cancers. Cancer.

2015;121(2):269-275. doi:10.1002/cncr.29041

14. Mavaddat N, Barrowdale D, Andrulis IL, et al. Pathology of Breast and Ovarian Cancers among BRCA1 and BRCA2 Mutation Carriers: Results from the Consortium of Investigators of Modifiers of BRCA1/2 (CIMBA). Cancer Epidemiology Biomarkers \& Prevention. 2012;21(1):134-147. doi:10.1158/1055-9965.EPI-11-0775

15. Reade CJ, McVey RM, Tone AA, et al. The Fallopian Tube as the Origin of High Grade Serous Ovarian Cancer: Review of a Paradigm Shift. Journal of Obstetrics and Gynaecology Canada. 2014;36(2):133-140. doi:10.1016/S1701-2163(15)30659-9

16. Gross AL, Kurman RJ, Vang R, Shih I-M, Visvanathan K. Precursor Lesions of HighGrade Serous Ovarian Carcinoma: Morphological and Molecular Characteristics. Journal of Oncology. 2010;2010:1-9. doi:10.1155/2010/126295

17. Lakhani SR. Pathology of Ovarian Cancers in BRCA1 and BRCA2 Carriers. Clinical Cancer Research. 2004;10(7):2473-2481. doi:10.1158/1078-0432.CCR-1029-3

18. Boyd J. Clinicopathologic Features of BRCA-Linked and Sporadic Ovarian Cancer. JAMA. 2000;283(17):2260. doi:10.1001/jama.283.17.2260

19. Kotsopoulos J, Rosen B, Fan I, et al. Ten-year survival after epithelial ovarian cancer is not associated with BRCA mutation status. Gynecologic Oncology. 2016;140(1):42-47. doi:10.1016/j.ygyno.2015.11.009

20. Prakash R, Zhang Y, Feng W, Jasin M. Homologous Recombination and Human Health: The Roles of BRCA1, BRCA2, and Associated Proteins. Cold Spring Harb Perspect Biol. 2015;7(4):a016600. doi:10.1101/cshperspect.a016600

21. Tarsounas M, Sung P. The antitumorigenic roles of BRCA1-BARD1 in DNA repair and replication. Nat Rev Mol Cell Biol. 2020;21(5):284-299. doi:10.1038/s41580-020-0218-Z

22. Roy R, Chun J, Powell SN. BRCA1 and BRCA2: different roles in a common pathway of genome protection. Nat Rev Cancer. 2012;12(1):68-78. doi:10.1038/nrc3181

23. Savage KI, Harkin DP. BRCA1, a 'complex' protein involved in the maintenance of genomic stability. FEBS J. 2015;282(4):630-646. doi:10.1111/febs.13150

24. Huen MSY, Sy SMH, Chen J. BRCA1 and its toolbox for the maintenance of genome integrity. Nat Rev Mol Cell Biol. 2010;11(2):138-148. doi:10.1038/nrm2831 
25. Ware MD, DeSilva D, Sinilnikova OM, Stoppa-Lyonnet D, Tavtigian SV, Mazoyer S. Does nonsense-mediated mRNA decay explain the ovarian cancer cluster region of the BRCA2 gene? Oncogene. 2006;25(2):323-328. doi:10.1038/sj.onc.1209033

26. Perrin-Vidoz L. The nonsense-mediated mRNA decay pathway triggers degradation of most BRCA1 mRNAs bearing premature termination codons. Human Molecular Genetics. 2002;11(23):2805-2814. doi:10.1093/hmg/11.23.2805

27. Huyton T, Bates PA, Zhang X, Sternberg MJE, Freemont PS. The BRCA1 C-terminal domain: structure and function. Mutation Research/DNA Repair. 2000;460(3-4):319-332. doi:10.1016/S0921-8777(00)00034-3

28. Girolimetti G, Perrone AM, Santini D, et al. BRCA-Associated Ovarian Cancer: From Molecular Genetics to Risk Management. BioMed Research International. 2014;2014:1-11. doi:10.1155/2014/787143

29. Singh AK, Yu X. Tissue-Specific Carcinogens as Soil to Seed BRCA1/2-Mutant Hereditary Cancers. Trends in Cancer. Published online April 2020:S2405803320301059. doi:10.1016/j.trecan.2020.03.004

30. Rebbeck TR, Mitra N, Wan F, et al. Association of Type and Location of BRCAl and BRCA2 Mutations With Risk of Breast and Ovarian Cancer. JAMA. 2015;313(13):1347. doi:10.1001/jama.2014.5985

31. Frank TS, Deffenbaugh AM, Reid JE, et al. Clinical Characteristics of Individuals With Germline Mutations in BRCA1 and BRCA2: Analysis of 10,000 Individuals. :11.

32. Ferla R, Calò V, Cascio S, et al. Founder mutations in BRCA1 and BRCA2 genes. Annals of Oncology. 2007;18:vi93-vi98. doi:10.1093/annonc/mdm234

33. Piek JMJ, van Diest PJ, Verheijen RHM. Ovarian Carcinogenesis: An Alternative Hypothesis. In: Coukos G, Berchuck A, Ozols R, eds. Ovarian Cancer. Vol 622. Advances in Experimental Medicine and Biology. Springer New York; 2008:79-87. doi:10.1007/9780-387-68969-2_7

34. Klotz DM, Wimberger P. Cells of origin of ovarian cancer: ovarian surface epithelium or fallopian tube? Arch Gynecol Obstet. 2017;296(6):1055-1062. doi:10.1007/s00404-0174529-z

35. Dubeau L. The Cell of Origin of Ovarian Epithelial Tumors and the Ovarian Surface Epithelium Dogma: Does the Emperor Have No Clothes? Gynecologic Oncology. 1999;72(3):437-442. doi:10.1006/gyno.1998.5275

36. Shih I-M, Wang Y, Wang T-L. The Origin of Ovarian Cancer Species and Precancerous Landscape. The American Journal of Pathology. 2021;191(1):26-39. doi:10.1016/j.ajpath.2020.09.006 
37. Kurman RJ, Shih I-M. The Dualistic Model of Ovarian Carcinogenesis. The American Journal of Pathology. 2016;186(4):733-747. doi:10.1016/j.ajpath.2015.11.011

38. Powell CB, Kenley E, Chen L, et al. Risk-Reducing Salpingo-Oophorectomy in BRCA Mutation Carriers: Role of Serial Sectioning in the Detection of Occult Malignancy. JCO. 2005;23(1):127-132. doi:10.1200/JCO.2005.04.109

39. Finch APM, Lubinski J, Møller P, et al. Impact of Oophorectomy on Cancer Incidence and Mortality in Women With a BRCA1 or BRCA2 Mutation. JCO. 2014;32(15):1547-1553. doi:10.1200/JCO.2013.53.2820

40. Callahan MJ, Crum CP, Medeiros F, et al. Primary Fallopian Tube Malignancies in BRCA Positive Women Undergoing Surgery for Ovarian Cancer Risk Reduction. JCO. 2007;25(25):3985-3990. doi:10.1200/JCO.2007.12.2622

41. Leeper K, Garcia R, Swisher E, Goff B, Greer B, Paley P. Pathologic Findings in Prophylactic Oophorectomy Specimens in High-Risk Women. Gynecologic Oncology. 2002;87(1):52-56. doi:10.1006/gyno.2002.6779

42. Medeiros F, Muto MG, Lee Y, et al. The Tubal Fimbria Is a Preferred Site for Early Adenocarcinoma in Women With Familial Ovarian Cancer Syndrome. American Journal of Surgical Pathology. 2006;30(2):230-236. doi:10.1097/01.pas.0000180854.28831.77

43. Kindelberger DW, Lee Y, Miron A, et al. Intraepithelial Carcinoma of the Fimbria and Pelvic Serous Carcinoma: Evidence for a Causal Relationship. The American Journal of Surgical Pathology. 2007;31(2):161-169. doi:10.1097/01.pas.0000213335.40358.47

44. Przybycin CG, Kurman RJ, Shih I-M. Are All Pelvic (Nonuterine) Serous Carcinomas of Tubal Origin? Am J Surg Pathol. 2010;34(10):10.

45. Kuhn E, Kurman RJ, Vang R, et al. TP53 mutations in serous tubal intraepithelial carcinoma and concurrent pelvic high-grade serous carcinoma-evidence supporting the clonal relationship of the two lesions. J Pathol. 2012;226(3):421-426. doi: $10.1002 /$ path.3023

46. Lee Y, Miron A, Drapkin R, et al. A candidate precursor to serous carcinoma that originates in the distal fallopian tube. J Pathol. 2007;211(1):26-35. doi:10.1002/path.2091

47. Marquez RT, Baggerly KA, Patterson AP, et al. Patterns of Gene Expression in Different Histotypes of Epithelial Ovarian Cancer Correlate with Those in Normal Fallopian Tube, Endometrium, and Colon. Clin Cancer Res. 2005;11(17):6116-6126. doi:10.1158/10780432.CCR-04-2509

48. Vang R, Shih I-M, Kurman RJ. Fallopian tube precursors of ovarian low- and high-grade serous neoplasms. Histopathology. 2013;62(1):44-58. doi:10.1111/his.12046

49. Scully R, Livingston DM. In search of the tumour-suppressor functions of BRCA1 and BRCA2. Nature. 2000;408(6811):429-432. doi:10.1038/35044000 
50. Smith SA, Easton DF, Evans DGR, Ponder BAJ. Allele losses in the region 17q12-21 in familial breast and ovarian cancer involve the wild-type chromosome. Nat Genet.

1992;2(2):128-131. doi:10.1038/ng1092-128

51. Gudmundsson J, Johannesdottir G, Bergthorsson JT, et al. Different Tumor Types from BRCA2 Carriers Show Wild-Type Chromosome Deletions on 13Q12-Q13. Published online 1995:4.

52. Merajver D, Frank S. Germline BRCA1 Mutations and Loss of the Wild-Type Allele in Tumors from Families with Early Onset Breast and Ovarian Cancer. Clinical Cancer Research. Published online 1995:7.

53. Venkitaraman AR. Linking the Cellular Functions of BRCA Genes to Cancer Pathogenesis and Treatment. Annu Rev Pathol Mech Dis. 2009;4(1):461-487.

doi:10.1146/annurev.pathol.3.121806.151422

54. Maxwell KN, Wubbenhorst B, Wenz BM, et al. BRCA locus-specific loss of heterozygosity in germline BRCA1 and BRCA2 carriers. Nat Commun. 2017;8(1):319. doi:10.1038/s41467-017-00388-9

55. Cousineau I, Belmaaza A. BRCA1 Haploinsufficiency, but not Heterozygosity for a BRCA1-truncating Mutation, Deregulates Homologous Recombination. Cell Cycle. 2007;6(8):962-971. doi:10.4161/cc.6.8.4105

56. Konishi H, Mohseni M, Tamaki A, et al. Mutation of a single allele of the cancer susceptibility gene BRCA1 leads to genomic instability in human breast epithelial cells. Proceedings of the National Academy of Sciences. 2011;108(43):17773-17778. doi:10.1073/pnas.1110969108

57. Sedic M, Skibinski A, Brown N, et al. Haploinsufficiency for BRCA1 leads to cell-typespecific genomic instability and premature senescence. Nat Commun. 2015;6(1):7505. doi:10.1038/ncomms 8505

58. Jeng Y-M, Cai-Ng S, Li A, et al. Brca1 heterozygous mice have shortened life span and are prone to ovarian tumorigenesis with haploinsufficiency upon ionizing irradiation. Oncogene. 2007;26(42):6160-6166. doi:10.1038/sj.onc.1210451

59. Venkitaraman AR. Cancer Suppression by the Chromosome Custodians, BRCA1 and BRCA2. Science. 2014;343(6178):1470-1475. doi:10.1126/science. 1252230

60. Green JA, Berns EMJJ, Coens C, et al. Alterations in the p53 pathway and prognosis in advanced ovarian cancer: A multi-factorial analysis of the EORTC Gynaecological Cancer group (study 55865). European Journal of Cancer. 2006;42(15):2539-2548. doi:10.1016/j.ejca.2006.06.015

61. Norquist BM, Garcia RL, Allison KH, et al. The molecular pathogenesis of hereditary ovarian carcinoma: Alterations in the tubal epithelium of women with BRCA1 and BRCA2 mutations. Cancer. 2010;116(22):5261-5271. doi:10.1002/cncr.25439 
62. Fathalla MF. INCESSANT OVULATION-A FACTOR IN OVARIAN NEOPLASIA ? The Lancet. 1971;298(7716):163. doi:10.1016/S0140-6736(71)92335-X

63. Ness RB, Cottreau C. Possible Role of Ovarian Epithelial Inflammation in Ovarian Cancer. JNCI Journal of the National Cancer Institute. 1999;91(17):1459-1467. doi:10.1093/jnci/91.17.1459

64. Trabert B, Tworoger SS, O'Brien KM, et al. The Risk of Ovarian Cancer Increases with an Increase in the Lifetime Number of Ovulatory Cycles: An Analysis from the Ovarian Cancer Cohort Consortium (OC3). Cancer Res. 2020;80(5):1210-1218. doi:10.1158/00085472.CAN-19-2850

65. Tone AA. Taking the Tube: From Normal Fallopian Tube Epithelium to Ovarian Highgrade Serous Carcinoma. Clinical Obstetrics and Gynecology. 2017;60(4):697-710. doi:10.1097/GRF.0000000000000313

66. Revelli A, Piane LD, Casano S, Molinari E, Massobrio M, Rinaudo P. Follicular fluid content and oocyte quality: from single biochemical markers to metabolomics. Reprod Biol Endocrinol. 2009;7(1):40. doi:10.1186/1477-7827-7-40

67. Huang H-S, Chu S-C, Hsu C-F, et al. Mutagenic, surviving and tumorigenic effects of follicular fluid in the context of p53 loss: initiation of fimbria carcinogenesis. CARCIN. 2015;36(11):1419-1428. doi:10.1093/carcin/bgv132

68. Lau A, Kollara A, John ES, et al. Altered expression of inflammation-associated genes in oviductal cells following follicular fluid exposure: Implications for ovarian carcinogenesis. Exp Biol Med (Maywood). 2014;239(1):24-32. doi:10.1177/1535370213508216

69. Duffy DM, Ko C, Jo M, Brannstrom M, Curry TE. Ovulation: Parallels With Inflammatory Processes. Endocrine Reviews. 2019;40(2):369-416. doi:10.1210/er.2018-00075

70. Savant S, Sriramkumar S, O'Hagan H. The Role of Inflammation and Inflammatory Mediators in the Development, Progression, Metastasis, and Chemoresistance of Epithelial Ovarian Cancer. Cancers. 2018;10(8):251. doi:10.3390/cancers10080251

71. Merritt MA, Green AC, Nagle CM, Webb PM, Australian Cancer Study (Ovarian Cancer) and Australian Ovarian Cancer Study Group. Talcum powder, chronic pelvic inflammation and NSAIDs in relation to risk of epithelial ovarian cancer. Int J Cancer. 2008;122(1):170176. doi:10.1002/ijc. 23017

72. Vercellini P, Crosignani P, Somigliana E, et al. The "incessant menstruation" hypothesis: a mechanistic ovarian cancer model with implications for prevention. Human Reproduction. 2011;26(9):2262-2273. doi:10.1093/humrep/der211

73. Narod SA, Sun P, Ghadirian P, et al. Tubal ligation and risk of ovarian cancer in carriers of BRCA1 or BRCA2 mutations: a case-control study. The Lancet. 2001;357(9267):14671470. doi:10.1016/S0140-6736(00)04642-0 
74. Rice MS, Hankinson SE, Tworoger SS. Tubal ligation, hysterectomy, unilateral oophorectomy, and risk of ovarian cancer in the Nurses' Health Studies. Fertility and Sterility. 2014;102(1):192-198.e3. doi:10.1016/j.fertnstert.2014.03.041

75. Antoniou AC, Rookus M, Andrieu N, et al. Reproductive and Hormonal Factors, and Ovarian Cancer Risk for BRCA1 and BRCA2 Mutation Carriers: Results from the International BRCA1/2 Carrier Cohort Study. Cancer Epidemiology Biomarkers \& Prevention. 2009;18(2):601-610. doi:10.1158/1055-9965.EPI-08-0546

76. Chan J, Sammel MD, Friebel TM, Gracia C, Rebbeck TR. Tubal ligation does not modify ovarian cancer risk in BRCA mutation carriers. Fertility and Sterility. 2015;104(3):e259. doi:10.1016/j.fertnstert.2015.07.813

77. Pearce CL, Templeman C, Rossing MA, et al. Association between endometriosis and risk of histological subtypes of ovarian cancer: a pooled analysis of case-control studies. The Lancet Oncology. 2012;13(4):385-394. doi:10.1016/S1470-2045(11)70404-1

78. Choi J-H, Wong AST, Huang H-F, Leung PCK. Gonadotropins and Ovarian Cancer. Endocrine Reviews. 2007;28(4):440-461. doi:10.1210/er.2006-0036

79. Kotsopoulos J, Lubinski J, Gronwald J, et al. Factors influencing ovulation and the risk of ovarian cancer in BRCA1 and BRCA2 mutation carriers: Ovulation and Ovarian Cancer in BRCA Mutation Carriers. Int J Cancer. 2015;137(5):1136-1146. doi:10.1002/ijc.29386

80. Mertens-Walker I, Baxter RC, Marsh DJ. Gonadotropin signalling in epithelial ovarian cancer. Cancer Letters. 2012;324(2):152-159. doi:10.1016/j.canlet.2012.05.017

81. King SM, Hilliard TS, Wu LY, Jaffe RC, Fazleabas AT, Burdette JE. The impact of ovulation on fallopian tube epithelial cells: evaluating three hypotheses connecting ovulation and serous ovarian cancer. Endocrine-Related Cancer. 2011;18(5):627-642. doi:10.1530/ERC-11-0107

82. Risch HA. Hormonal Etiology of Epithelial Ovarian Cancer, With a Hypothesis Concerning the Role of Androgens and Progesterone. JNCI Journal of the National Cancer Institute. 1998;90(23):1774-1786. doi:10.1093/jnci/90.23.1774

83. Edmondson RJ, Monaghan JM, Davies BR. The human ovarian surface epithelium is an androgen responsive tissue. Br J Cancer. 2002;86(6):879-885. doi:10.1038/sj.bjc.6600154

84. Zhu H, Zhu X, Zheng L, Hu X, Sun L, Zhu X. The role of the androgen receptor in ovarian cancer carcinogenesis and its clinical implications. Oncotarget. 2017;8(17):29395-29405. doi:10.18632/oncotarget.12561

85. Colina JA, Zink KE, Eliadis K, et al. Fallopian Tube-Derived Tumor Cells Induce Testosterone Secretion from the Ovary, Increasing Epithelial Proliferation and Invasion. Cancers. 2021;13(8):1925. doi:10.3390/cancers13081925 
86. Barry JA, Azizia MM, Hardiman PJ. Risk of endometrial, ovarian and breast cancer in women with polycystic ovary syndrome: a systematic review and meta-analysis. Human Reproduction Update. 2014;20(5):748-758. doi:10.1093/humupd/dmu012

87. Olsen CM, Green AC, Whiteman DC, Sadeghi S, Kolahdooz F, Webb PM. Obesity and the risk of epithelial ovarian cancer: A systematic review and meta-analysis. European Journal of Cancer. 2007;43(4):690-709. doi:10.1016/j.ejca.2006.11.010

88. Piek JMJ, van Diest PJ, Zweemer RP, et al. Dysplastic changes in prophylactically removed Fallopian tubes of women predisposed to developing ovarian cancer. J Pathol. 2001;195(4):451-456. doi:10.1002/path.1000

89. Piek JMJ, Verheijen RHM, Menko FH, et al. Expression of differentiation and proliferation related proteins in epithelium of prophylactically removed ovaries from women with a hereditary female adnexal cancer predisposition: Differentiation and proliferation related proteins of prophylactically removed ovaries. Histopathology. 2003;43(1):26-32. doi:10.1046/j.1365-2559.2003.01654.x

90. Modugno F, Laskey R, Smith AL, Andersen CL, Haluska P, Oesterreich S. Hormone response in ovarian cancer: time to reconsider as a clinical target? Endocrine-Related Cancer. 2012;19(6):R255-R279. doi:10.1530/ERC-12-0175

91. Bu S-Z, Yin D-L, Ren X-H, et al. Progesterone induces apoptosis and up-regulation of p53 expression in human ovarian carcinoma cell lines. American Cancer Society. Published online 1997:7.

92. Rodriguez GC. Progestin-Induced Apoptosis in the Macaque Ovarian Epithelium: Differential Regulation of Transforming Growth Factor-. CancerSpectrum Knowledge Environment. 2002;94(1):50-60. doi:10.1093/jnci/94.1.50

93. Wu N-Y, Huang H-S, Chao TH, et al. Progesterone Prevents High-Grade Serous Ovarian Cancer by Inducing Necroptosis of p53-Defective Fallopian Tube Epithelial Cells. Cell Reports. 2017;18(11):2557-2565. doi:10.1016/j.celrep.2017.02.049

94. Rodriguez GC, Kauderer J, Hunn J, et al. Phase II Trial of Chemopreventive Effects of Levonorgestrel on Ovarian and Fallopian Tube Epithelium in Women at High Risk for Ovarian Cancer: An NRG Oncology Group/GOG Study. Cancer Prev Res. 2019;12(6):401412. doi:10.1158/1940-6207.CAPR-18-0383

95. Diep CH, Daniel AR, Mauro LJ, Knutson TP, Lange CA. Progesterone action in breast, uterine, and ovarian cancers. Journal of Molecular Endocrinology. 2015;54(2):R31-R53. doi:10.1530/JME-14-0252

96. Syed V, Ulinski G, Mok SC, Yiu GK, Ho S-M. Expression of Gonadotropin Receptor and Growth Responses to Key Reproductive Hormones in Normal and Malignant Human Ovarian Surface Epithelial Cells. Published online 2001:10. 
97. Widschwendter M, Rosenthal AN, Philpott S, et al. The sex hormone system in carriers of BRCA1/2 mutations: a case-control study. The Lancet Oncology. 2013;14(12):1226-1232. doi:10.1016/S1470-2045(13)70448-0

98. Kim O, Park EY, Kwon SY, et al. Targeting progesterone signaling prevents metastatic ovarian cancer. Proc Natl Acad Sci USA. Published online December 1, 2020:202013595. doi:10.1073/pnas.2013595117

99. Daly MB, Pal T, Berry MP, et al. Genetic/Familial High-Risk Assessment: Breast, Ovarian, and Pancreatic, Version 2.2021, NCCN Clinical Practice Guidelines in Oncology. Journal of the National Comprehensive Cancer Network. 2021;19(1):77-102. doi:10.6004/jnccn.2021.0001

100. Jacobson M, Bernardini M, Sobel ML, Kim RH, McCuaig J, Allen L. SOGC CLINICAL PRACTICE GUIDELINE: No. 366-Gynaecologic Management of Hereditary Breast and Ovarian Cancer. Journal of Obstetrics and Gynaecology Canada. 2018;40(11):1497-1510. doi:10.1016/j.jogc.2018.05.046

101. the SEOM Hereditary Cancer Working Group, González-Santiago S, Ramón y Cajal T, et al. SEOM clinical guidelines in hereditary breast and ovarian cancer (2019). Clin Transl Oncol. 2020;22(2):193-200. doi:10.1007/s12094-019-02262-0

102. Paluch-Shimon S, Cardoso F, Sessa C, et al. Prevention and screening in BRCA mutation carriers and other breast/ovarian hereditary cancer syndromes: ESMO Clinical Practice Guidelines for cancer prevention and screening. Annals of Oncology. 2016;27:v103-v110. doi:10.1093/annonc/mdw327

103. Walker M, Jacobson M, Sobel M. Management of ovarian cancer risk in women with BRCA1/2 pathogenic variants. CMAJ. 2019;191(32):E886-E893. doi:10.1503/cmaj.190281

104. Domchek SM. Association of Risk-Reducing Surgery in BRCA1 or BRCA2 Mutation Carriers With Cancer Risk and Mortality. JAMA. 2010;304(9):967. doi:10.1001/jama.2010.1237

105. Rebbeck TR, Lynch HT, Neuhausen SL, et al. Prophylactic Oophorectomy in Carriers of BRCA1 or BRCA2 Mutations. N Engl J Med. 2002;346(21):1616-1622. doi:10.1056/NEJMoa012158

106. Rebbeck TR, Kauff ND, Domchek SM. Meta-analysis of Risk Reduction Estimates Associated With Risk-Reducing Salpingo-oophorectomy in BRCA1 or BRCA2 Mutation Carriers. JNCI Journal of the National Cancer Institute. 2009;101(2):80-87. doi:10.1093/jnci/djn442

107. Eleje GU, Eke AC, Ezebialu IU, Ikechebelu JI, Ugwu EO, Okonkwo OO. Risk-reducing bilateral salpingo-oophorectomy in women with BRCA1 or BRCA2 mutations. Cochrane Gynaecological, Neuro-oncology and Orphan Cancer Group, ed. Cochrane Database of Systematic Reviews. Published online August 24, 2018. doi:10.1002/14651858.CD012464.pub2 
108. AlHilli MM, Pederson HJ. Controversies in Hereditary Cancer Management. Obstetrics \& Gynecology. 2021;137(5):941-955. doi:10.1097/AOG.0000000000004364

109. Berliner JL, Cummings SA, Boldt Burnett B, Ricker CN. Risk assessment and genetic counseling for hereditary breast and ovarian cancer syndromes-Practice resource of the National Society of Genetic Counselors. Jrnl of Gene Coun. 2021;30(2):342-360. doi:10.1002/jgc4.1374

110. the Hereditary Breast Cancer Clinical Study Group, Metcalfe K, Eisen A, et al. International trends in the uptake of cancer risk reduction strategies in women with a BRCA1 or BRCA2 mutation. Br J Cancer. 2019;121(1):15-21. doi:10.1038/s41416-0190446-1

111. Rosenthal AN, Fraser LSM, Philpott S, et al. Evidence of Stage Shift in Women Diagnosed With Ovarian Cancer During Phase II of the United Kingdom Familial Ovarian Cancer Screening Study. JCO. 2017;35(13):1411-1420. doi:10.1200/JCO.2016.69.9330

112. Skates SJ, Greene MH, Buys SS, et al. Early Detection of Ovarian Cancer using the Risk of Ovarian Cancer Algorithm with Frequent CA125 Testing in Women at Increased Familial Risk - Combined Results from Two Screening Trials. Clin Cancer Res. 2017;23(14):36283637. doi:10.1158/1078-0432.CCR-15-2750

113. Narod SA. Oral Contraceptives and the Risk of Breast Cancer in BRCA1 and BRCA2 Mutation Carriers. CancerSpectrum Knowledge Environment. 2002;94(23):1773-1779. doi:10.1093/jnci/94.23.1773

114. Milne RL, Antoniou AC. Modifiers of breast and ovarian cancer risks for BRCA1 and BRCA2 mutation carriers. Endocrine-Related Cancer. 2016;23(10):T69-T84. doi:10.1530/ERC-16-0277

115. Lee A, Mavaddat N, Wilcox AN, et al. BOADICEA: a comprehensive breast cancer risk prediction model incorporating genetic and nongenetic risk factors. Genet Med. 2019;21(8):1708-1718. doi:10.1038/s41436-018-0406-9

116. Friebel TM, Domchek SM, Rebbeck TR. Modifiers of cancer risk in BRCA1 and BRCA2 mutation carriers: systematic review and meta-analysis. J Natl Cancer Inst. 2014;106(6):dju091. doi:10.1093/jnci/dju091

117. Babic A, Sasamoto N, Rosner BA, et al. Association Between Breastfeeding and Ovarian Cancer Risk. JAMA Oncol. Published online April 2, 2020:e200421. doi:10.1001/jamaoncol.2020.0421

118. Kotsopoulos J, Gronwald J, McCuaig JM, et al. Breastfeeding and the risk of epithelial ovarian cancer among women with a BRCA1 or BRCA2 mutation. Gynecologic Oncology. Published online September 2020:S0090825820339470. doi:10.1016/j.ygyno.2020.09.037 
119. Troisi R, Bjørge T, Gissler M, et al. The role of pregnancy, perinatal factors and hormones in maternal cancer risk: a review of the evidence. J Intern Med. 2018;283(5):430-445. doi:10.1111/joim.12747

120. Wentzensen N, Poole EM, Trabert B, et al. Ovarian Cancer Risk Factors by Histologic Subtype: An Analysis From the Ovarian Cancer Cohort Consortium. JCO. 2016;34(24):2888-2898. doi:10.1200/JCO.2016.66.8178

121. Yang H, Dai H, Li L, et al. Age at menarche and epithelial ovarian cancer risk: A metaanalysis and Mendelian randomization study. Cancer Med. 2019;8(8):4012-4022. doi:10.1002/cam4.2315

122. Tsilidis KK, Allen NE, Key TJ, et al. Oral contraceptive use and reproductive factors and risk of ovarian cancer in the European Prospective Investigation into Cancer and Nutrition. Br J Cancer. 2011;105(9):1436-1442. doi:10.1038/bjc.2011.371

123. Gronwald J, Byrski T, Huzarski T, et al. Influence of selected lifestyle factors on breast and ovarian cancer risk in BRCA1 mutation carriers from Poland. Breast Cancer Res Treat. 2006;95(2):105-109. doi:10.1007/s10549-005-9051-5

124. Perri T, Lifshitz D, Sadetzki S, et al. Fertility treatments and invasive epithelial ovarian cancer risk in Jewish Israeli BRCA1 or BRCA2 mutation carriers. Fertility and Sterility. 2015;103(5):1305-1312. doi:10.1016/j.fertnstert.2015.02.011

125. Collaborative Group on Epidemiological Studies of Ovarian Cancer. Menopausal hormone use and ovarian cancer risk: individual participant meta-analysis of 52 epidemiological studies. The Lancet. 2015;385(9980):1835-1842. doi:10.1016/S0140-6736(14)61687-1

126. Huber D, Seitz S, Kast K, Emons G, Ortmann O. Hormone replacement therapy in BRCA mutation carriers and risk of ovarian, endometrial, and breast cancer: a systematic review. $J$ Cancer Res Clin Oncol. Published online April 22, 2021. doi:10.1007/s00432-021-03629-z

127. Kotsopoulos J, Lubinski J, Neuhausen SL, et al. Hormone replacement therapy and the risk of ovarian cancer in BRCA1 and BRCA2 mutation carriers. Gynecologic Oncology. 2006;100(1):83-88. doi:10.1016/j.ygyno.2005.07.110

128. Vicus D, Rosen B, Lubinski J, et al. Tamoxifen and the risk of ovarian cancer in BRCA1 mutation carriers. Gynecologic Oncology. 2009;115(1):135-137. doi:10.1016/j.ygyno.2009.06.012

129. Rizzuto I, Behrens RF, Smith LA. Risk of ovarian cancer in women treated with ovarian stimulating drugs for infertility. Cochrane Gynaecological, Neuro-oncology and Orphan Cancer Group, ed. Cochrane Database of Systematic Reviews. Published online August 13, 2013. doi:10.1002/14651858.CD008215.pub2

130. Gronwald J, Glass K, Rosen B, et al. Treatment of infertility does not increase the risk of ovarian cancer among women with a BRCA1 or BRCA2 mutation. Fertility and Sterility. 2016;105(3):781-785. doi:10.1016/j.fertnstert.2015.11.034 
131. Coletta AM, Peterson SK, Gatus LA, et al. Diet, weight management, physical activity and Ovarian \& Breast Cancer Risk in women with BRCA1/2 pathogenic Germline gene variants: systematic review. Hered Cancer Clin Pract. 2020;18(1):5. doi:10.1186/s13053020-0137-1

132. Collaborative Group on Epidemiological Studies of Ovarian Cancer. Ovarian cancer and smoking: individual participant meta-analysis including 28114 women with ovarian cancer from 51 epidemiological studies. The Lancet Oncology. 2012;13(9):946-956.

doi:10.1016/S1470-2045(12)70322-4

133. Ko K-P, Kim SJ, Huzarski T, et al. The association between smoking and cancer incidence in BRCAI and BRCA2 mutation carriers: Smoking and BRCA -cancer risk. Int J Cancer. 2018;142(11):2263-2272. doi:10.1002/ijc.31257

134. Lauby-Secretan B, Scoccianti C, Loomis D, Grosse Y, Bianchini F, Straif K. Body Fatness and Cancer - Viewpoint of the IARC Working Group. N Engl J Med. 2016;375(8):794798. doi:10.1056/NEJMsr1606602

135. KConFab Investigators, HEBON Investigators, GEMO Study Collaborators, et al. Mendelian randomisation study of height and body mass index as modifiers of ovarian cancer risk in 22,588 BRCA1 and BRCA2 mutation carriers. Br J Cancer. 2019;121(2):180-192. doi:10.1038/s41416-019-0492-8

136. Kim S, Lubinski J, Huzarski T, et al. Weight gain and the risk of ovarian cancer in BRCA1 and BRCA2 mutation carriers. Molecular Genetics and Metabolism. 2021;132:S357-S358. doi:10.1016/S1096-7192(21)00626-0

137. Shafiei F, Salari-Moghaddam A, Milajerdi A, Larijani B, Esmaillzadeh A. Coffee and caffeine intake and risk of ovarian cancer: a systematic review and meta-analysis. Int J Gynecol Cancer. 2019;29(3):579-584. doi:10.1136/ijgc-2018-000102

138. Speroff L, Fritz MA. Clinical Gynecologic Endocrinology and Infertility. Eighth. S.1. : Lippincott Williams and Wilkins; 2011.

139. Stanczyk FZ. Pharmacokinetics and Potency of Progestins used for Hormone Replacement Therapy and Contraception. Reviews in Endocrine \& Metabolic Disorders. Published online 2002:14.

140. Davtyan C. Four generations of progestins in oral contraceptives. Proceedings of UCLA Healthcare. 2012;16:3.

141. Kuhl H. Pharmacokinetics of oestrogens and progestogens. Maturitas. 1990;12(3):171-197. doi:10.1016/0378-5122(90)90003-O

142. Bitzer J. Progestogens in Contraception. In: Carp HJA, ed. Progestogens in Obstetrics and Gynecology. Springer International Publishing; 2015:111-127. doi:10.1007/978-3-31914385-9_8 
143. Lobo RA, Stanczyk, FZ. New knowledge in the physiology of hormonal contraceptives. American Journal of Obstetrics and Gynecology. 1994;170(5):1499-1507. doi:10.1016/S0002-9378(94)05011-8

144. Kiley J, Hammond C. Combined Oral Contraceptives: A Comprehensive Review. Clinical Obstetrics \& Gynecology. 2007;50(4):868-877. doi:10.1097/GRF.0b013e318159c06a

145. Kuhl H. Pharmacology of estrogens and progestogens: influence of different routes of administration. Climacteric. 2005;8(sup1):3-63. doi:10.1080/13697130500148875

146. Stanczyk FZ, Hapgood JP, Winer S, Mishell DR. Progestogens Used in Postmenopausal Hormone Therapy: Differences in Their Pharmacological Properties, Intracellular Actions, and Clinical Effects. Endocrine Reviews. 2013;34(2):171-208. doi:10.1210/er.2012-1008

147. De Leo V, Musacchio MC, Cappelli V, Piomboni P, Morgante G. Hormonal contraceptives: pharmacology tailored to women's health. Hum Reprod Update. 2016;22(5):634-646. doi:10.1093/humupd/dmw016

148. Stanczyk FZ, Archer DF. Gestodene: A review of its pharmacology, potency and tolerability in combined contraceptive preparations. Contraception. 2014;89(4):242-252. doi:10.1016/j.contraception.2013.12.003

149. Sitruk-Ware R. Pharmacological profile of progestins. Maturitas. 2004;47(4):277-283. doi:10.1016/j.maturitas.2004.01.001

150. Royer PA, Jones KP. Progestins for Contraception: Modern Delivery Systems and Novel Formulations. Clinical Obstetrics and Gynecology. 2014;57(4):644-658. doi:10.1097/GRF.0000000000000072

151. Regidor P-A. Clinical relevance in present day hormonal contraception. Hormone Molecular Biology and Clinical Investigation. 2019;37(1). doi:10.1515/hmbci-2018-0030

152. Golobof A, Kiley J. The Current Status of Oral Contraceptives: Progress and Recent Innovations. Semin Reprod Med. 2016;34(03):145-151. doi:10.1055/s-0036-1572546

153. United Nations. Contraceptive Use by Method 2019: Data Booklet. UN; 2019. doi:10.18356/1bd58a10-en

154. Gerstman BB, Gross TP, Kennedy DL, Bennett RC, Tomita DK, Stadel BV. Trends in the content and use of oral contraceptives in the United States, 1964-88. Am J Public Health. 1991;81(1):90-96. doi:10.2105/AJPH.81.1.90

155. Rotermann M, Dunn S, Black A. Oral contraceptive use among women aged 15 to 49 : Results from the Canadian Health Measures Survey. Health Rep. 2015;26(10):21-28.

156. Hall KS, Trussell J. Types of combined oral contraceptives used by US women. Contraception. 2012;86(6):659-665. doi:10.1016/j.contraception.2012.05.017 
157. Mazza D, Harrison C, Taft A, et al. Current contraceptive management in Australian general practice: an analysis of BEACH data. Medical Journal of Australia. 2012;197(2):110-114. doi:10.5694/mja11.11599

158. Dericks-Tan JSE, Gudacker V, Taubert H-D. Influence of oral contraceptives on integrated secretion of gonadotropins. Contraception. 1992;46(4):369-377. doi:10.1016/00107824(92)90099-F

159. Gaspard UJ, Remus MA, Gillain D, Duvivier J, Demey E, FranchimontO P. PLASMA HORMONE LEVELS IN WOMEN RECEIVING NEW ORAL CONTRACEPTIVES CONTAINING ETHINYL ESTRADIOL PLUS LEVONORGESTREL OR DESOGESTREL. 1983;27(6):14.

160. Scott JA, Brenner PF, Kletzky OA, Mishell DR. Factors affecting pituitary gonadotropin function in users of oral contraceptive steroids. American Journal of Obstetrics and Gynecology. 1978;130(7):817-821. doi:10.1016/0002-9378(78)90015-7

161. Trussell J. Contraceptive failure in the United States. Contraception. 2011;83(5):397-404. doi:10.1016/j.contraception.2011.01.021

162. Winner B, Buckel C, Secura GM. Effectiveness of Long-Acting Reversible Contraception. $n$ engl j med. Published online 2012:10.

163. Wildemeersch D, Dhont M, Weyers S, Temmerman M. Invited lecture to be held at the IX European Congress of Pediatric and Adolescent Gynecology in Florence (to be published in the Journal of Pediatric and Adolescent Gynecology). Thursday, 5 December 2002 : 5.20 $5.40 \mathrm{pm} .: 13$.

164. Sutton B. Long-Acting Reversible Contraceptive Device Regulations: Lessons for Canada. Published online 2019.

165. Black A. Contraception in Canada: A Nod to the Past and Look Into the Future. Journal of Obstetrics and Gynaecology Canada. 2019;41:S305-S308. doi:10.1016/j.jogc.2019.09.001

166. Economidis MA, Mishell Jr DR. Pharmacological female contraception: an overview of past and future use. Expert Opinion on Investigational Drugs. 2005;14(4):449-456. doi:10.1517/13543784.14.4.449

167. Sivin I, Nash H, Waldman S. Jadelle® Levonorgestrel Rod Implants: A Summary of Scientific Data and Lessons Learned from Programmatic Experience. Population Council; 2002. doi:10.31899/cbr1.1000

168. Darney P, Patel A, Rosen K, Shapiro LS, Kaunitz AM. Safety and efficacy of a single-rod etonogestrel implant (Implanon): results from 11 international clinical trials. Fertility and Sterility. 2009;91(5):1646-1653. doi:10.1016/j.fertnstert.2008.02.140 
169. Brache V, Alvarez-Sanchez F, Faundes A, Tejada A, Cochon L. Ovarian endocrine function through five years of continuous treatment with NORPLANT® subdermal contraceptive implants. Contraception. 1990;41(2):169-177. doi:10.1016/0010-7824(90)90145-L

170. Makarainen L. Ovarian Function During the Use of a Single Contraceptive Implant: Implanon Compared with Norplant. Fertility and Sterility. 1998;69(4):714-721. doi:10.1016/S0015-0282(98)00015-6

171. Huber J. Pharmacokinetics of Implanon®. Contraception. 1998;58(6):85S-90S. doi:10.1016/S0010-7824(98)00120-6

172. Croxatto HB, Mäkäräinen L. The pharmacodynamics and efficacy of Implanon®. Contraception. 1998;58(6):91S-97S. doi:10.1016/S0010-7824(98)00118-8

173. Inki P. Long-term use of the levonorgestrel-releasing intrauterine system. Contraception. 2007;75(6):S161-S166. doi:10.1016/j.contraception.2006.12.016

174. Nelson A, Massoudi, MPH N. New developments in intrauterine device use: focus on the US. OAJC. 2016;Volume 7:127-141. doi:10.2147/OAJC.S85755

175. Strasser J, Borkowski L, Couillard M, Allina A, Wood S. LONG-ACTING REVERSIBLE CONTRACEPTION: Overview of Research \& Policy in the United States. Published online 2016:77.

176. Bayer HealthCare Pharmaceuticals Incl. Mirena [package insert].

177. Apter D, Gemzell-Danielsson K, Hauck B, Rosen K, Zurth C. Pharmacokinetics of two low-dose levonorgestrel-releasing intrauterine systems and effects on ovulation rate and cervical function: pooled analyses of phase II and III studies. Fertility and Sterility. 2014;101(6):1656-1662.e4. doi:10.1016/j.fertnstert.2014.03.004

178. Bayer HealthCare Pharmaceuticals Inc. Kyleena [package insert].

179. Bayer HealthCare Pharmaceuticals Inc. Skyla [package insert].

180. Lähteenmäki P. The levonorgestrel intrauterine system in contraception. Steroids. 2000;65(10-11):693-697. doi:10.1016/S0039-128X(00)00176-8

181. Luukkainen T. Levonorgestrel-Releasing Intrauterine Device. Ann NY Acad Sci. 1991;626(1 Frontiers in):43-49. doi:10.1111/j.1749-6632.1991.tb37898.x

182. Nilsson CG, Lähteenmäki PLA, Luukkainen T. Ovarian function in amenorrheic and menstruating users of a levonorgestrel-releasing intrauterine device**This work was undertaken as part of the contraceptive development program sponsored and coordinated by the International Committee for Contraception Research of the Population Council, Inc., New York, New York. The financial support provided by the Ford Foundation, the Rockefeller Foundation, the International Development Research Centre of Canada, and The Geo. J. Hecht Fund is gratefully acknowledged. The content does not necessarily 
reflect the policy of any of the funding sources. Fertility and Sterility. 1984;41(1):52-55. doi:10.1016/S0015-0282(16)47540-0

183. Nelson A, Massoudi, MPH N. New developments in intrauterine device use: focus on the US. OAJC. 2016;Volume 7:127-141. doi:10.2147/OAJC.S85755

184. Sivin I. IUDs are Contraceptives, Not Abortifacients: A Comment on Research and Belief. Studies in Family Planning. 1989;20(6):355. doi:10.2307/1966438

185. Intrauterine contraception_Background and device types - UpToDate. Published online January 24, 2020:28.

186. WHO Scientific Group. Mechanism of action, safety and efficacy of intrauterine devices. World Health Organ Tech Rep Ser. 1987;753:1-91.

187. El-Habashi M, El-Sahwi S, Gawish S, Osman M. Effect of lippes loop on sperm recovery from human fallopian tubes. Contraception. 1980;22(5):549-555. doi:10.1016/00107824(80)90107-9

188. Sagroglu N. PHAGOCYTOSIS OF SPERMATOZOA IN THE UTERINE CAVITY OF WOMAN USING INTRAUTERINE DEVICE: Obstetrical \& Gynecological Survey. 1971;26(10):691-692. doi:10.1097/00006254-197110000-00003

189. Critchley HO, Wang H, Jones RL, et al. Morphological and functional features of endometrial decidualization following long-term intrauterine levonorgestrel delivery. Human Reproduction. 1998;13(5):1218-1224. doi:10.1093/humrep/13.5.1218

190. Lewis RA, Taylor D, Natavio MF, Melamed A, Felix J, Mishell D. Effects of the levonorgestrel-releasing intrauterine system on cervical mucus quality and sperm penetrability. Contraception. 2010;82(6):491-496. doi:10.1016/j.contraception.2010.06.006

191. Xiao B, Zeng T, Wu S, Sun H, Xiao N. Effect of levonorgestrel-releasing intrauterine device on hormonal profile and menstrual pattern after long-term use. Contraception. 1995;51(6):359-365. doi:10.1016/0010-7824(95)00102-G

192. Wang SL, Wu SC, Xin XM, Chen JH, Gao J. Three years' experience with levonorgestrelreleasing intrauterine device and Norplant-2 implants: a randomized comparative study. Adv Contracept. 1992;8(2):105-114. doi:10.1007/BF01849748

193. Nilsson CG, Lähteenmäki P, Luukkainen T. Levonorgestrel plasma concentrations and hormone profiles after insertion and after one year of treatment with a levonorgestrel-IUD. Contraception. 1980;21(3):225-233. doi:10.1016/0010-7824(80)90003-7

194. Barbosa I, Bakos O, Olsson S-E, Odlind V, Johansson EDB. Ovarian function during use of a levonorgestrel-releasing IUD. Contraception. 1990;42(1):51-66. doi:10.1016/00107824(90)90092-A 
195. Goldsmith A, Toppozada M. Long-Acting Contraception. Vol 11.; 1983. Accessed July 6, 2021. http://journals.lww.com/00019616-200103000-00010

196. Luukkainen T, Lähteenmäki P, Toivonen J. Levonorgestrel-Releasing Intrauterine Device. Annals of Medicine. 1990;22(2):85-90. doi:10.3109/07853899009147248

197. Seleem S, Hills FA, SaIem HT, El-Nashar EM, Chard T. Mechanism of action of the intrauterine contraceptive device: evidence for a specific biochemical deficiency in the endometrium. Human Reproduction. 1996;11(6):1220-1222.

doi:10.1093/oxfordjournals.humrep.a019359

198. Cuadros A, Hirsch JG. Copper on Intrauterine Devices Stimulates Leukocyte Exudation. Science. 1972;175(4018):175-176. doi:10.1126/science.175.4018.175

199. Holland MK, White IG. Heavy metals and human spermatozoa. III. The toxicity of copper ions for spermatozoa. Contraception. 1988;38(6):685-695. doi:10.1016/00107824(88)90050-9

200. Roblero L, Guadarrama A, Lopez T, Zegers-Hochschild F. Effect of copper ion on the motility, viability, acrosome reaction and fertilizing capacity of human spermatozoa in vitro. Reprod Fertil Dev. 1996;8(5):871. doi:10.1071/RD9960871

201. Kesserü E, Camacho-Ortega P. INFLUENCE OF METALS ON IN VITRO SPERM MIGRATION IN THE HUMAN CERVICAL MUCUS: Obstetrical \& Gynecological Survey. 1973;28(2):132-133. doi:10.1097/00006254-197302000-00021

202. Elstein M, Ferrer K. THE EFFECT OF A COPPER-RELEASING INTRAUTERINE DEVICE ON SPERM PENETRATION IN HUMAN CERVICAL MUCUS IN VITRO. Reproduction. 1973;32(1):109-111. doi:10.1530/jrf.0.0320109

203. Hefnawi F, Serour G. INFLUENCE OF THE COPPER IUD AND THE LIPPES LOOP m SPERM MIGRKJION IN THE HUMAN CERVICAL bRJCUS. 1975;11(5):7.

204. Larsson B, Ljung B, Hamberger L. The influence of copper on the in vitro motility of the human Fallopian tube. American Journal of Obstetrics and Gynecology. 1976;125(5):682690. doi:10.1016/0002-9378(76)90794-8

205. Sivin I, ed. The Copper T 380 Intrauterine Device: A Summary of Scientific Data. Population Council; 1992.

206. Jain J, Dutton C, Nicosia A, Wajszczuk C, Bode FR, Mishell DR. Pharmacokinetics, ovulation suppression and return to ovulation following a lower dose subcutaneous formulation of Depo-Provera®. Contraception. 2004;70(1):11-18. doi:10.1016/j.contraception.2004.01.011

207. Rivera R, Yacobson I, Grimes D. The mechanism of action of hormonal contraceptives and intrauterine contraceptive devices. American Journal of Obstetrics and Gynecology. 1999;181(5):1263-1269. doi:10.1016/S0002-9378(99)70120-1 
208. Petta CA, Faundes A, Dunson TR, et al. Timing of onset of contraceptive effectiveness in Depo-Provera users: Part I. Changes in cervical mucus. Fertility and Sterility. 1998;69(2):252-257. doi:10.1016/S0015-0282(97)00477-9

209. International Agency for Research on Cancer, ed. Hormonal Contraception and PostMenopausal Hormonal Therapy: This Publication Represents the Views and Expert Opinions of an IARC Working Group on the Evaluation of Carcinogenic Risks to Humans, Which Met in Lyon, 2 - 9 June 1998. IARC; 1999.

210. Collaborative Group on Epidemiological Studies of Ovarian Cancer. Ovarian cancer and oral contraceptives: collaborative reanalysis of data from 45 epidemiological studies including 23257 women with ovarian cancer and 87303 controls. The Lancet. 2008;371(9609):303-314. doi:10.1016/S0140-6736(08)60167-1

211. Franceschi S, Parazzini F, Negri E, et al. Pooled analysis of 3 european case-control studies of epithelial ovarian cancer: III. Oral contraceptive use. Int J Cancer. 1991;49(1):61-65. doi:10.1002/ijc.2910490112

212. Bosetti C, Negri E, Trichopoulos D, et al. Long-term effects of oral contraceptives on ovarian cancer risk. Int J Cancer. 2002;102(3):262-265. doi:10.1002/ijc.10696

213. Havrilesky LJ, Moorman PG, Lowery WJ, et al. Oral Contraceptive Pills as Primary Prevention for Ovarian Cancer: A Systematic Review and Meta-analysis. Obstetrics \& Gynecology. 2013;122(1):139-147. doi:10.1097/AOG.0b013e318291c235

214. Petitti DB. Combination Estrogen-Progestin Oral Contraceptives. The New England Journal of Medicine. Published online 2003:8.

215. Spona J, Feichtinger W, Kindermann Ch, Wünsch C, Brill K. Inhibition of ovulation by an oral contraceptive containing $100 \mu \mathrm{g}$ levonorgestrel in combination with $20 \mu \mathrm{g}$ ethinylestradiol. Contraception. 1996;54(5):299-304. doi:10.1016/S0010-7824(96)00183-7

216. Crosignani PG, Testa G, Vegetti W, Parazzini F. Ovarian activity during regular oral contraceptive use. Contraception. 1996;54(5):271-273. doi:10.1016/S0010-7824(96)001783

217. Shafrir AL, Schock H, Poole EM, et al. A prospective cohort study of oral contraceptive use and ovarian cancer among women in the United States born from 1947 to 1964. Cancer Causes Control. 2017;28(5):371-383. doi:10.1007/s10552-017-0876-0

218. Schildkraut JM. Impact of Progestin and Estrogen Potency in Oral Contraceptives on Ovarian Cancer Risk. CancerSpectrum Knowledge Environment. 2002;94(1):32-38. doi:10.1093/jnci/94.1.32

219. Pike MC, Pearce CL, Peters R, Cozen W, Wan P, Wu AH. Hormonal factors and the risk of invasive ovarian cancer: a population-based case-control study. Fertility and Sterility. 2004;82(1):186-195. doi:10.1016/j.fertnstert.2004.03.013 
220. Ness RB, Grisso JA, Klapper J, et al. Risk of ovarian cancer in relation to estrogen and progestin dose and use characteristics of oral contraceptives. SHARE Study Group. Steroid Hormones and Reproductions. Am J Epidemiol. 2000;152(3):233-241. doi:10.1093/aje/152.3.233

221. Faber MT, Jensen A, Frederiksen K, et al. Oral contraceptive use and impact of cumulative intake of estrogen and progestin on risk of ovarian cancer. Cancer Causes Control. 2013;24(12):2197-2206. doi:10.1007/s10552-013-0296-8

222. Rosenberg L, Palmer JR, Zauber AG, et al. A Case-Control Study of Oral Contraceptive Use and Invasive Epithelial Ovarian Cancer. American Journal of Epidemiology. 1994;139(7):654-661. doi:10.1093/oxfordjournals.aje.a117055

223. Sanderson M, Hendrix NW. Oral Contraceptives and Epithelial Ovarian Cancer. The Journal of Reproductive Medicine. 2000;45(9):8.

224. Phung MT, Lee AW, Wu AH, et al. Depot-medroxyprogesterone acetate use is associated with decreased risk of ovarian cancer: the mounting evidence of a protective role of progestins. Cancer Epidemiol Biomarkers Prev. Published online February 22, 2021:cebp.EPI-20-1355-E.2020. doi:10.1158/1055-9965.EPI-20-1355

225. Wilailak S, Vipupinyo C, Suraseranivong V, et al. Depot medroxyprogesterone acetate and epithelial ovarian cancer: a multicentre case-control study: DMPA and epithelial ovarian cancer. BJOG: An International Journal of Obstetrics \& Gynaecology. 2012;119(6):672677. doi:10.1111/j.1471-0528.2012.03298.x

226. Urban M, Banks E, Egger S, et al. Injectable and Oral Contraceptive Use and Cancers of the Breast, Cervix, Ovary, and Endometrium in Black South African Women: Case-Control Study. Franco EL, ed. PLoS Med. 2012;9(3):e1001182. doi:10.1371/journal.pmed.1001182

227. Iversen L, Fielding S, Lidegaard Ø, Mørch LS, Skovlund CW, Hannaford PC. Association between contemporary hormonal contraception and ovarian cancer in women of reproductive age in Denmark: prospective, nationwide cohort study. BMJ. Published online September 26, 2018:k3609. doi:10.1136/bmj.k3609

228. Huang Z, Gao Y, Wen W, et al. Contraceptive methods and ovarian cancer risk among Chinese women: A report from the Shanghai Women's Health Study: Contraceptive methods and ovarian cancer risk. Int J Cancer. 2015;137(3):607-614. doi:10.1002/ijc.29412

229. Wheeler LJ, Desanto K, Teal SB, Sheeder J, Guntupalli SR. Intrauterine Device Use and Ovarian Cancer Risk: A Systematic Review and Meta-analysis. Obstetrics \& Gynecology. 2019;134(4):791-800. doi:10.1097/AOG.0000000000003463

230. Balayla J, Gil Y, Lasry A, Mitric C. Ever-use of the intra-uterine device and the risk of ovarian cancer. Journal of Obstetrics and Gynaecology. Published online October 12, 2020:1-6. doi:10.1080/01443615.2020.1789960 
231. Jareid M, Thalabard J-C, Aarflot M, Bøvelstad HM, Lund E, Braaten T. Levonorgestrelreleasing intrauterine system use is associated with a decreased risk of ovarian and endometrial cancer, without increased risk of breast cancer. Results from the NOWAC Study. Gynecologic Oncology. 2018;149(1):127-132. doi:10.1016/j.ygyno.2018.02.006

232. Soini T, Hurskainen R, Grénman S, Mäenpää J, Paavonen J, Pukkala E. Impact of levonorgestrel-releasing intrauterine system use on the cancer risk of the ovary and fallopian tube. Acta Oncologica. 2016;55(11):1281-1284.

doi:10.1080/0284186X.2016.1175660

233. Yang J, Sasamoto N, Babic A, et al. Intrauterine device use and risk of ovarian cancer: Results from the New England Case-Control study and Nurses' Health Studies. Int J Cancer. Published online March 17, 2021:ijc.33531. doi:10.1002/ijc.33531

234. Tworoger SS, Fairfield KM, Colditz GA, Rosner BA, Hankinson SE. Association of Oral Contraceptive Use, Other Contraceptive Methods, and Infertility with Ovarian Cancer Risk. American Journal of Epidemiology. 2007;166(8):894-901. doi:10.1093/aje/kwm157

235. Cibula D, Zikan M, Dusek L, Majek O. Oral contraceptives and risk of ovarian and breast cancers in BRCA mutation carriers: a meta-analysis. Expert Rev Anticancer Ther. 2011;11(8):1197-1207. doi:10.1586/era.11.38

236. Iodice $\mathrm{S}$, Barile $\mathrm{M}$, Rotmensz N, et al. Oral contraceptive use and breast or ovarian cancer risk in BRCA1/2 carriers: A meta-analysis. European Journal of Cancer. 2010;46(12):2275-2284. doi:10.1016/j.ejca.2010.04.018

237. Moorman PG, Havrilesky LJ, Gierisch JM, et al. Oral Contraceptives and Risk of Ovarian Cancer and Breast Cancer Among High-Risk Women: A Systematic Review and MetaAnalysis. JCO. 2013;31(33):4188-4198. doi:10.1200/JCO.2013.48.9021

238. Schrijver LH, Antoniou AC, Olsson H, et al. Oral contraceptive use and ovarian cancer risk for BRCA1/2 mutation carriers: an international cohort study. American Journal of Obstetrics and Gynecology. Published online January 2021:S0002937821000387. doi:10.1016/j.ajog.2021.01.014

239. Cibula D, Widschwendter M, Zikan M, Dusek L. Underlying mechanisms of ovarian cancer risk reduction after tubal ligation: Ovarian cancer risk after tubal ligation. Acta Obstetricia et Gynecologica Scandinavica. 2011;90(6):559-563. doi:10.1111/j.16000412.2011.01114.x

240. Finch A, Beiner M, Lubinski J, et al. Salpingo-oophorectomy and the risk of ovarian, fallopian tube, and peritoneal cancers in women with a BRCA1 or BRCA2 Mutation. JAMA. 2006;296(2):185-192. doi:10.1001/jama.296.2.185

241. Marchetti C, De Felice F, Boccia S, et al. Hormone replacement therapy after prophylactic risk-reducing salpingo-oophorectomy and breast cancer risk in BRCA1 and BRCA2 mutation carriers: A meta-analysis. Critical Reviews in Oncology/Hematology. 2018;132:111-115. doi:10.1016/j.critrevonc.2018.09.018 
242. Black A, Guilbert E, Costescu D, et al. Canadian Contraception Consensus (Part 3 of 4): Chapter 8 - Progestin-Only Contraception. Journal of Obstetrics and Gynaecology Canada. 2016;38(3):279-300. doi:10.1016/j.jogc.2015.12.003

243. Black A, Guilbert E, Costescu D, et al. Canadian Contraception Consensus (Part 3 of 4): Chapter 7: Intrauterine Contraception. Journal of Obstetrics and Gynaecology Canada. 2016;38(2):182-222. doi:10.1016/j.jogc.2015.12.002

244. McLaughlin JR, Risch HA, Lubinski J, et al. Reproductive risk factors for ovarian cancer in carriers of BRCA1 or BRCA2 mutations: a case-control study. Lancet Oncol. 2007;8(1):2634. doi:10.1016/S1470-2045(06)70983-4

245. King LA, Michels KA, Graubard BI, Trabert B. Trends in oral contraceptive and intrauterine device use among reproductive-aged women in the US from 1999 to 2017. Cancer Causes Control. Published online March 10, 2021. doi:10.1007/s10552-021-014108

246. Black A, Rouhani S, Cook J. CONTRACEPTIVE USE AND TEN-YEAR TRENDS IN CANADIAN WOMEN OF REPRODUCTIVE AGE. Journal of Obstetrics and Gynaecology Canada. 2019;41(5):711-712. doi:10.1016/j.jogc.2019.02.164

247. Sexual and Reproductive Health Services, England (Contraception) 2019/20. Accessed July 28, 2021. https://digital.nhs.uk/data-and-information/publications/statistical/sexual-andreproductive-health-services/2019-20/methods-of-contraception

248. Burnhill MS. The rise and fall and rise of the IUD. Am J Gynecol Health. 1989;3(3-S):6-10.

249. Mosher WD, Moreau C, Lantos H. Trends and determinants of IUD use in the USA, 20022012. Hum Reprod. 2016;31(8):1696-1702. doi:10.1093/humrep/dew117

250. Kaunitz AM. Long-acting injectable contraception with depot medroxyprogesterone acetate. American Journal of Obstetrics and Gynecology. 1994;170(5):1543-1549. doi:10.1016/S0002-9378(12)91813-X

251. Ortiz ME, Croxatto HB. Copper-T intrauterine device and levonorgestrel intrauterine system: biological bases of their mechanism of action. Contraception. 2007;75(6):S16-S30. doi:10.1016/j.contraception.2007.01.020

252. Felix AS, Gaudet MM, Vecchia CL, et al. Intrauterine devices and endometrial cancer risk: A pooled analysis of the Epidemiology of Endometrial Cancer Consortium: Intrauterine devices and endometrial cancer risk. Int J Cancer. 2015;136(5):E410-E422. doi:10.1002/ijc.29229

253. Ness RB, Dodge RC, Edwards RP, Baker JA, Moysich KB. Contraception Methods, Beyond Oral Contraceptives and Tubal Ligation, and Risk of Ovarian Cancer. Annals of Epidemiology. 2011;21(3):188-196. doi:10.1016/j.annepidem.2010.10.002 
254. Huber D, Seitz S, Kast K, Emons G, Ortmann O. Use of oral contraceptives in BRCA mutation carriers and risk for ovarian and breast cancer: a systematic review. Arch Gynecol Obstet. 2020;301(4):875-884. doi:10.1007/s00404-020-05458-w

255. Fortner RT, Ose J, Merritt MA, et al. Reproductive and hormone-related risk factors for epithelial ovarian cancer by histologic pathways, invasiveness and histologic subtypes: Results from the EPIC cohort: Risk factors for ovarian cancer by subtype. Int J Cancer. 2015;137(5):1196-1208. doi:10.1002/ijc.29471

256. Merritt MA, De Pari M, Vitonis AF, Titus LJ, Cramer DW, Terry KL. Reproductive characteristics in relation to ovarian cancer risk by histologic pathways. Human Reproduction. 2013;28(5):1406-1417. doi:10.1093/humrep/des466

257. Yu S, Lee M, Shin S, Park J. Apoptosis induced by progesterone in human ovarian cancer cell line SNU-840. J Cell Biochem. 2001;82(3):445-451. doi:10.1002/jcb.1171

258. Rodriguez GC, Walmer DK, Cline M, et al. Effect of Progestin on the Ovarian Epithelium of Macaques: Cancer Prevention Through Apoptosis? Published online 1998:6.

259. Lovett JL, Chima MA, Wexler JK, et al. Oral contraceptives cause evolutionarily novel increases in hormone exposure. Evolution, Medicine, and Public Health. 2017;2017(1):97108. doi:10.1093/emph/eox009

260. Lockhat FB, Emembolu JE, Konje JC. Serum and peritoneal fluid levels of levonorgestrel in women with endometriosis who were treated with an intrauterine contraceptive device containing levonorgestrel. Fertility and Sterility. 2005;83(2):398-404. doi:10.1016/j.fertnstert.2004.07.961

261. Bahamondes L, Fernandes A, Monteiro I, Bahamondes MV. Long-acting reversible contraceptive (LARCs) methods. Best Practice \& Research Clinical Obstetrics \& Gynaecology. 2020;66:28-40. doi:10.1016/j.bpobgyn.2019.12.002

262. Hunter DJ, Troy L. Reproducibility of Oral Contraceptive Histories and Validity of Hormone Composition Reported in a Cohort of US Women. Published online 1997:6.

263. Kotsopoulos J, Hereditary Breast Cancer Clinical Study Group, Lubinski J, et al. Timing of oral contraceptive use and the risk of breast cancer in BRCA1 mutation carriers. Breast Cancer Res Treat. 2014;143(3):579-586. doi:10.1007/s10549-013-2823-4

264. Kotsopoulos J, Gronwald J, Karlan BY, et al. Hormone Replacement Therapy After Oophorectomy and Breast Cancer Risk Among BRCAl Mutation Carriers. JAMA Oncol. 2018;4(8):1059. doi:10.1001/jamaoncol.2018.0211

265. Kathleen Cuningham Foundation Consortium for Research into Familial Breast Cancer (kConFab), Nolan E, Vaillant F, et al. RANK ligand as a potential target for breast cancer prevention in BRCA1-mutation carriers. Nat Med. 2016;22(8):933-939. doi:10.1038/nm.4118 
266. Sigl V, Owusu-Boaitey K, Joshi PA, et al. RANKL/RANK control Brca1 mutation-driven mammary tumors. Cell Res. 2016;26(7):761-774. doi:10.1038/cr.2016.69

267. Kotsopoulos J, Singer C, Narod SA. Can we prevent BRCA1-associated breast cancer by RANKL inhibition? Breast Cancer Res Treat. 2017;161(1):11-16. doi:10.1007/s10549-0164029-z

268. Shamseddin M, De Martino F, Constantin C, et al. Contraceptive progestins with androgenic properties stimulate breast epithelial cell proliferation. EMBO Mol Med. Published online May 27, 2021. doi:10.15252/emmm.202114314

269. Conz L, Mota BS, Bahamondes L, et al. Levonorgestrel-releasing intrauterine system and breast cancer risk: A systematic review and meta-analysis. Acta Obstet Gynecol Scand. 2020;99(8):970-982. doi:10.1111/aogs.13817

270. Modugno F, Moslehi R, Ness RB, et al. Reproductive factors and ovarian cancer risk in Jewish BRCA1 and BRCA2 mutation carriers (United States). Published online 2003:8.

271. Rutter JL, Wacholder S, Chetrit A, et al. Gynecologic Surgeries and Risk of Ovarian Cancer in Women With BRCA1 and BRCA2 Ashkenazi Founder Mutations: An Israeli Population-Based Case-Control Study. Journal of the National Cancer Institute. 2003;95(14):7. 
Appendices

Appendix 1. Research Questionnaire for the Risk Factor Analysis of Breast and Ovarian Cancer

\section{RESEARCH QUESTIONNAIRE FOR}

\section{A STUDY OF BREAST AND OVARIAN CANCER}

\section{IN HIGH RISK FAMILIES}

This questionnaire is part of a research study to improve our understanding of the prevention and treatment of hereditary breast and ovarian cancers.

Family/Individual \#:

Date of Birth: $\frac{1}{\mathrm{~mm}}-\frac{}{\text { yyyy }}$ Age:

Date Completed: $\frac{1}{\mathrm{~mm}} \frac{1}{\mathrm{dd}} \frac{}{\mathrm{yyyy}}$ 
1. Have you ever been pregnant?

No $\rightarrow$ Go to question 5 .

Yes

2. Please consider all pregnancies in order from first to last. Give year of pregnancy. Indicate the outcome of each pregnancy. For live-born children, try to recall how long you breast-fed each child and indicate months of breast-feeding (if not breast-fed, enter ' 0 ').

\begin{tabular}{|c|c|c|c|c|c|c|c|}
\hline & \multicolumn{7}{|c|}{ PREGNANCY } \\
\hline$\#$ & $\begin{array}{c}\text { Year of } \\
\text { Pregnancy }\end{array}$ & $\begin{array}{l}\text { Length of } \\
\text { Pregnancy } \\
\text { (In Weeks) }\end{array}$ & $\begin{array}{l}\text { Pregnancy Outcome } \\
\text { (Live, Still, Miscarriage } \\
\text { Abortion, Twins, etc.) }\end{array}$ & $\begin{array}{c}\text { Live Birth } \\
\text { Caesarean } \\
\text { Section? } \\
\text { (Yes, No) }\end{array}$ & $\begin{array}{l}\text { Live Born } \\
\text { (Month/Year } \\
\text { of Birth) }\end{array}$ & $\begin{array}{l}\text { Birth Weight } \\
\text { (lbs.) }\end{array}$ & $\begin{array}{l}\text { Months } \\
\text { of Breast- } \\
\text { Feeding }\end{array}$ \\
\hline 1 & & & & & & & \\
\hline 2 & & & & & & & \\
\hline 3 & & & & & & & \\
\hline 4 & & & & & & & \\
\hline 5 & & & & & & & \\
\hline 6 & & & & & & & \\
\hline 7 & & & & & & & \\
\hline 8 & & & & & & & \\
\hline 9 & & & & & & & \\
\hline 10 & & & & & & & \\
\hline
\end{tabular}

3. Did you ever have difficulty breast-feeding?

\begin{tabular}{|c|c|c|}
\hline No & & \\
\hline Yes & $\begin{array}{c}\rightarrow \text { Reason: _ poor milk production } \\
\text { _ premature infant }\end{array}$ & $\begin{array}{l}\text { _ pain } \\
\text { _ mastitis (breast inflammation) }\end{array}$ \\
\hline
\end{tabular}

Circle which child (1-10 in chart above) you experienced this with:

$$
\begin{array}{llllllllll}
1 & 2 & 3 & 4 & 5 & 6 & 7 & 8 & 9 & 10
\end{array}
$$

4. Have you ever taken medication to stop milk production?

No

Yes $\rightarrow$ Name of medication (if known):

Method:_ injections or pills

Circle with which child (1-10 in preceding chart) you took this medication.

$$
\begin{array}{llllllllll}
1 & 2 & 3 & 4 & 5 & 6 & 7 & 8 & 9 & 10
\end{array}
$$


5. How old were you when you had your first menstrual period? YEARS OLD

6. How would you describe your menstrual cycle? By regular we mean that the start of your period was predictable within 5 days. (Please check one)

My periods are/were always regular.

My periods are/were usually regular.

My periods are/were never regular.

7. How many days apart are your menstrual periods? That is, from the start of one period to the start of the next period. For example, many women have cycles of 28 days. (Please check one)
-28 days
29 days
30 days
31 days
32 days Other $\rightarrow$
days

8. Do you currently have menstrual periods? That is, have you had a menstrual period within the last year? Please answer "Yes" if you are currently pregnant, breast-feeding or taking hormones which temporarily stop your menses (Mirena IUD or birth control pills).

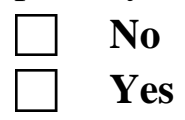

9. Have your periods stopped completely?

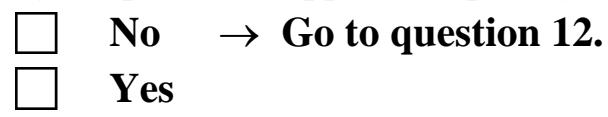

10. How old were you when your periods stopped completely? YEARS OLD

11. What was the reason your periods stopped? (Select one only)

$\square \quad$ Natural Menopause (change of life)

Hysterectomy (uterus removed only, ovaries not removed)

One ovary removed only

Both ovaries removed only

Fallopian tubes and ovaries removed

Uterus, fallopian tubes, and ovaries removed

Medication / Chemotherapy

Other (please specify):

12. Have you ever seen a doctor for a problem of difficulty in getting pregnant or in carrying a pregnancy, such as several miscarriages?

\section{No $\rightarrow$ Go to question 15 .}

Yes $\rightarrow$ What reason did the doctor give to explain why you had trouble getting or staying pregnant? (Please check all that apply.)

_ no problem was found

_ problem with cervix

_ problem with ovaries

_ problem with fallopian tubes

_ partner has fertility problem

_ other (please specify): 
13. Have you ever taken medication to increase your chances of becoming pregnant?

No

Yes $\rightarrow$ Name of medication(s):

For how many months did you take this medication? months What years did you take this medication?

14. Have you ever received fertility treatment such as in vitro fertilization/Embryo Transfer (IVF/ET) to help you get pregnant?

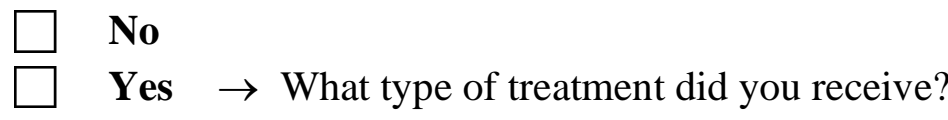

15a. Have you ever used birth control pills, Norplant (implants), Mirena/IUD, or Depo-Provera (injections) to prevent pregnancy or for any other reason?

No $\rightarrow$ Go to question 16 .

Yes $\rightarrow$ Can you describe the times?

\begin{tabular}{|l|l|l|l|l|l|}
\hline \multicolumn{1}{|c|}{ Method } & Name of Medication & $\begin{array}{c}\text { Starting } \\
\text { Year }\end{array}$ & $\begin{array}{c}\text { Ending } \\
\text { Year }\end{array}$ & $\begin{array}{c}\text { Length of Time } \\
\text { Used in Years }\end{array}$ \\
\hline 1 & $\begin{array}{l}\square \text { Pills } \square \text { Implants } \\
\square \text { Mirena/IUD } \\
\square \text { Injections } \square \text { Other }\end{array}$ & & \\
\hline 2 & $\begin{array}{l}\square \text { Pills } \square \text { Implants } \\
\square \text { Mirena/IUD } \\
\square \text { Injections } \square \text { Other }\end{array}$ & & & \\
\hline 3 & $\begin{array}{l}\square \text { Pills } \square \text { Implants } \\
\square \text { Mirena/IUD } \\
\square \text { Injections } \square \text { Other } \\
\square \text { Pills } \square \text { Implants } \\
\square \text { Mirena/IUD } \\
\square \text { Injections } \square \text { Other }\end{array}$ & & & \\
\hline
\end{tabular}

15b. Are you currently using birth control pills, Norplant (implants), Mirena/IUD, or Depo-Provera (injections)?

No

Yes 
16a. Have you ever taken hormone replacement therapy for menopause (i.e. estrogen, progesterone)?

No $\rightarrow$ Go to question 17 .

Yes $\rightarrow$ Complete table below:

\begin{tabular}{|c|c|c|c|c|c|}
\hline & Method & $\begin{array}{c}\text { Name of } \\
\text { Medication }\end{array}$ & $\begin{array}{c}\text { Starting } \\
\text { Year }\end{array}$ & $\begin{array}{c}\text { Ending } \\
\text { Year }\end{array}$ & $\begin{array}{c}\text { Length of Time } \\
\text { Used in Years }\end{array}$ \\
\hline 1 & $\begin{array}{l}\square \text { Pills } \square \text { Skin Patches } \\
\square \text { Vaginal Suppositories } \\
\square \text { Gel } \quad \square \text { Cream } \\
\square \text { Ring } \square \text { Injections } \\
\square \text { Other }\end{array}$ & & & & \\
\hline 2 & $\begin{array}{l}\square \text { Pills } \quad \square \text { Skin Patches } \\
\square \text { Vaginal Suppositories } \\
\square \text { Gel } \quad \square \text { Cream } \\
\square \text { Ring } \square \text { Injections } \\
\square \text { Other }\end{array}$ & & & & \\
\hline 3 & $\begin{array}{l}\square \text { Pills } \square \text { Skin Patches } \\
\square \text { Vaginal Suppositories } \\
\square \text { Gel } \quad \square \text { Cream } \\
\square \text { Ring } \square \text { Injections } \\
\square \text { Other }\end{array}$ & & & & \\
\hline 4 & $\begin{array}{l}\square \text { Pills } \quad \square \text { Skin Patches } \\
\square \text { Vaginal Suppositories } \\
\square \text { Gel } \quad \square \text { Cream } \\
\square \text { Ring } \square \text { Injections } \\
\square \text { Other }\end{array}$ & & & & \\
\hline
\end{tabular}

16b. Are you currently taking hormone replacement therapy?

No

Yes $\rightarrow$ Name of medication: dose?

\section{SECTION II - Breast Cancer Screening/Prevention}

17. Have you ever taken any drugs for the prevention of breast cancer (i.e. drugs taken before any diagnosis of breast cancer)?

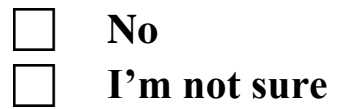

$\square$ Yes $\rightarrow$ Name of Drug: Tamoxifen (Nolvadex) Raloxifene (Evista)

_ Aromasin (Exemestane) Femara (Letrozole)

__ Arimidex (Anastrozole)

Other (please name):

Number of pills per day:

Date started: (mm / yyyy) to (mm / yyyy) 
18. Approximately how many mammograms have you had in your lifetime?

I have had mammograms. If you do not recall the exact number, please provide a range.

Age at first mammogram?

Age at last mammogram? years old.

19. Have you ever had any abnormalities detected by mammogram?

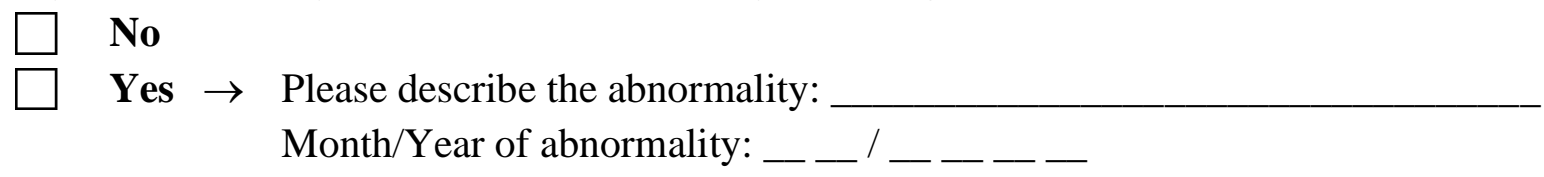

20. Have you ever had MRI screening of your breasts?

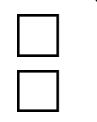

No
Yes

$\rightarrow$ Age at first MRI:

Month/Year of first MRI:

How many MRIs have you had?

Where were they done:

Were there any abnormalities detected by MRI? $\square$ Yes $\square$ No

If yes, please describe:

Year of abnormality:

Month/Year of your last MRI:

21. Have you ever had a breast biopsy?

No

Yes $\rightarrow$ Number of biopsies:

Month/Year of last biopsy:

What was the result of the biopsy? (Check all that apply)

$\begin{array}{lll}\text { normal } & \text { DCIS } & \text { - }{ }^{\text {atypical hyperplasia }} \\ \text { cancer } & \text { LCIS } & \text { fibroadenoma } \\ \text { other (please specify) } & \text { - }\end{array}$

22. Have you undergone surgery at any time in order to prevent breast cancer (e.g. preventive removal of the breasts)? NOTE: The surgery is only preventive if you've never previously been diagnosed with cancer in that breast.

No

Yes $\rightarrow$ What year? Month (if known)?

Which breast? Left

Right

Both

Procedure: Total mastectomy (nipple and areola removed) Subcutaneous mastectomy (nipple and areola preserved)

23. Have you ever had breast implants or breast reconstruction?<smiles>C1=C2CCC2=CC1</smiles>
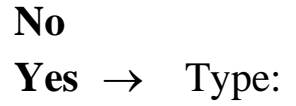

_ Saline Other Silicone TRAM-flap

Year of surgery:

24. Have you ever had breast reduction?

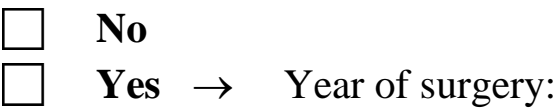


25. Have you ever been diagnosed with breast cancer?

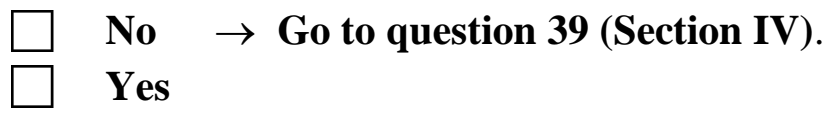

Check one $\rightarrow$ I have had cancer in $\square$ one breast

OR $\square$ both breasts

We would like to discuss your first diagnosis.

What year was it?

Which breast?

Month (if known)?

Right

\section{Type of Cancer (check one):}

It was an invasive cancer.

It was a non-invasive cancer (early stage breast cancer).

$\rightarrow \quad$ Was it Ductal Carcinoma In-situ (DCIS)?

$\rightarrow \quad$ Was it Lobular Carinoma In-situ (LCIS)?

It was both invasive and non-invasive.

I'm not sure.

Treatment

Did you have surgery for the first breast cancer?

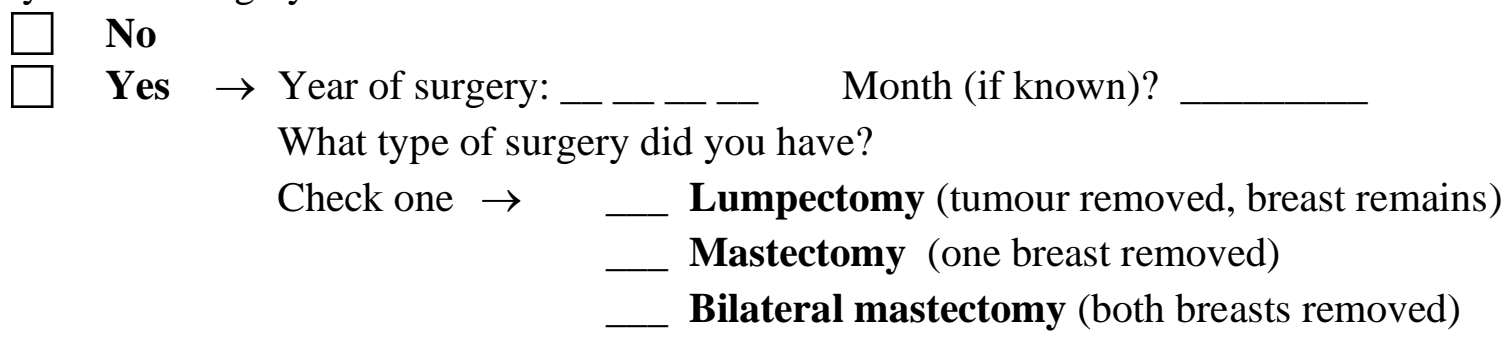

Detection

How was the lump first detected?

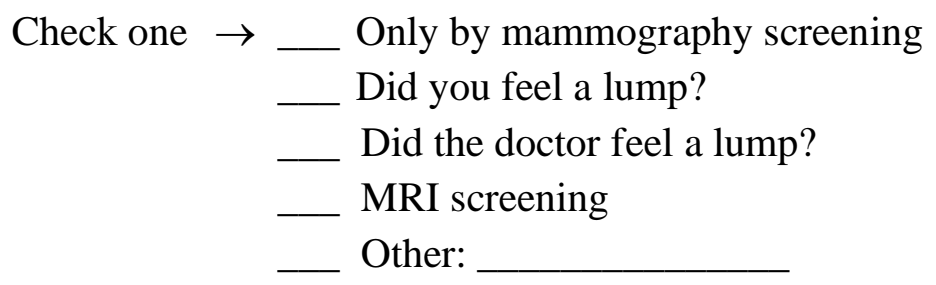

26. Did you receive chemotherapy for the first breast cancer?

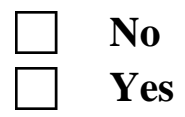

27. Did you receive radiation therapy for the first breast cancer?

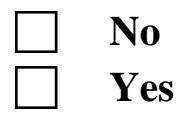

28. Did you receive Tamoxifen (Nolvadex) for the first breast cancer? 
No

Yes $\rightarrow$ Number of pills per day:

From: (year) to (year)

29. Did you receive any other drugs for the first breast cancer?

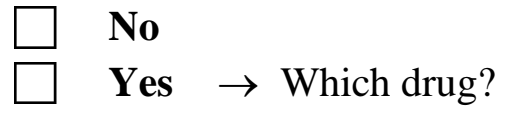

$$
\begin{aligned}
& \square \text { Femara (letrozole) } \\
& \text { Aromasin (exemestane) } \\
& \text { Arimidex (anastrazole) } \\
& \text { Other: }
\end{aligned}
$$

Number of pills per day:

From: (year) to (year)

30. Has your breast cancer come back in the same breast (local recurrence) or spread elsewhere (distant recurrence/metastasis)?

$\begin{array}{ll}\square & \text { No } \\ \square & \text { Yes } \rightarrow \text { Date: }\end{array}$ (mm / yyyy)

Site of recurrence:

Treatment for recurrence:

31. Have you had further surgery in the affected breast (such as complete mastectomy or reconstruction)?

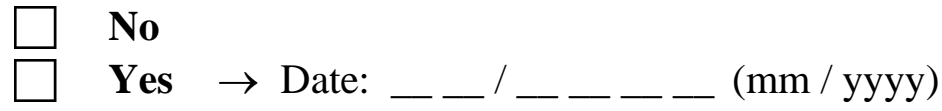

Type of surgery:

\section{IF YOU DID NOT HAVE A SECOND BREAST CANCER, GO TO SECTION IV QUESTION 39.}

32. We would like to discuss your second diagnosis (This does NOT include a recurrence, i.e. a cancer in the same breast as the previous breast cancer).

What year was it?

Which breast?

$\square$ Left

Month (if known)?

\section{Right}

Type of Cancer (check all that apply):

It was an invasive cancer.

It was a non-invasive cancer (early stage breast cancer).

$\rightarrow \quad$ Was it Ductal Carcinoma In-situ (DCIS)?

$\rightarrow \quad$ Was it Lobular Carinoma In-situ (LCIS)?

It was both invasive and non-invasive.

I'm not sure. 


\section{$\underline{\text { Treatment }}$}

Did you have surgery for the second breast cancer?

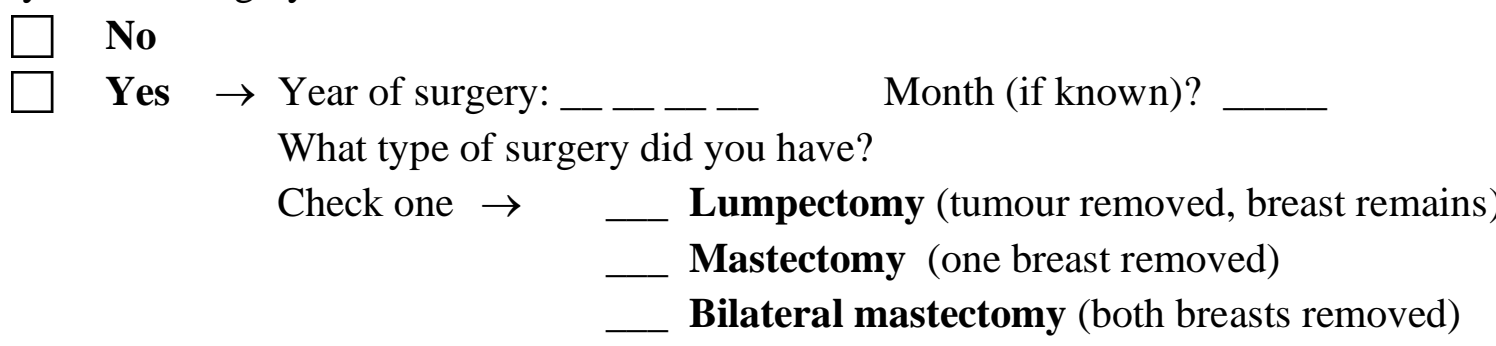

Detection

How was the lump first detected?

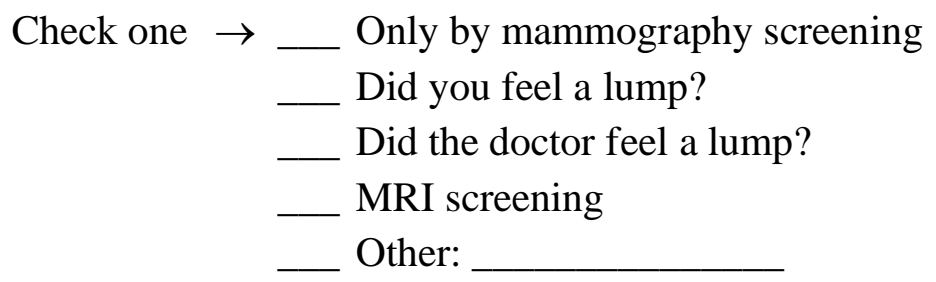

33. Did you receive chemotherapy for the second breast cancer?

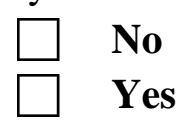

34. Did you receive radiation therapy for the second breast cancer?

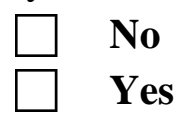

35. Did you receive Tamoxifen (Nolvadex) for the second breast cancer?

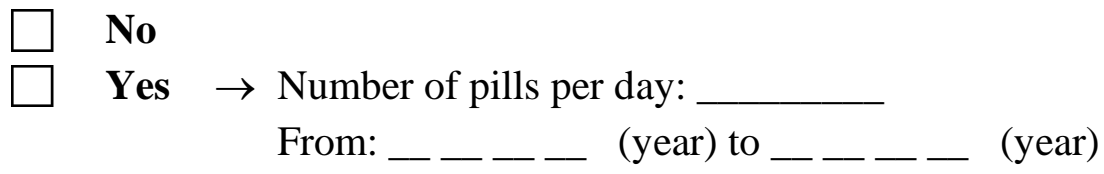

36. Did you receive any other drugs for the second breast cancer?

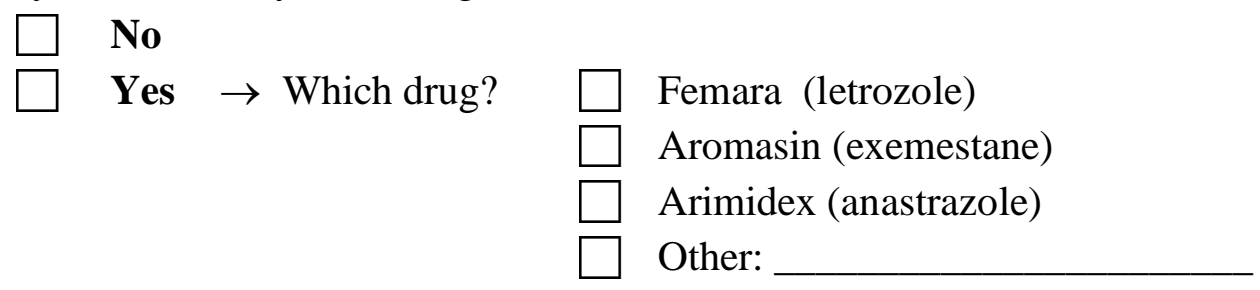

Number of pills per day:

From: _ _ _ _ (year) to _ _ _ _ _ (year)

37. Has your breast cancer come back in the same breast (local recurrence) or spread elsewhere (distant recurrence/metastasis)?

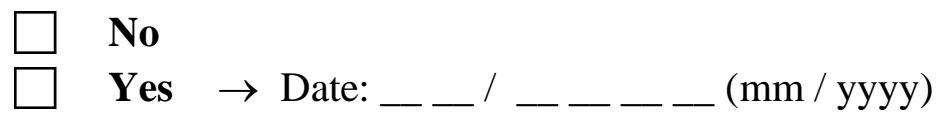

Site of recurrence:

Treatment for recurrence:

38. Have you had further surgery in the affected breast (such as complete mastectomy or reconstruction)?

No

$$
\text { Yes } \rightarrow \text { Date: }
$$
(mm / уууу)

Type of surgery: 
39. Have you ever had a trans-vaginal ultrasound (internal ultrasound done through the vagina)?

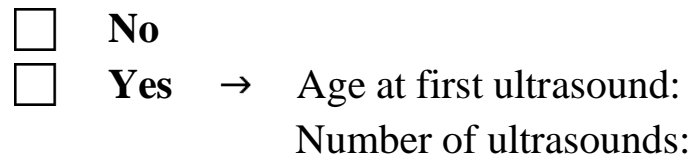

Abnormalities detected by ultrasound: $\square$ Yes $\square$ No

If Yes: Describe:

Year of abnormality:

Year of last trans-vaginal ultrasound:

40. Have you ever had a blood test for CA125 (marker for ovarian cancer)?

$\square$ Yes $\rightarrow$ Age at first CA125:

Number of CA125 tests:

Abnormalities detected:

No Elevated

Don't Know

Year of abnormality:

Year of the last CA125:

41. Have you had one or both of your ovaries removed (oophorectomy) for reasons other than ovarian cancer? (e.g. preventative measures, fibroids, cyst, scar tissue, or pain)

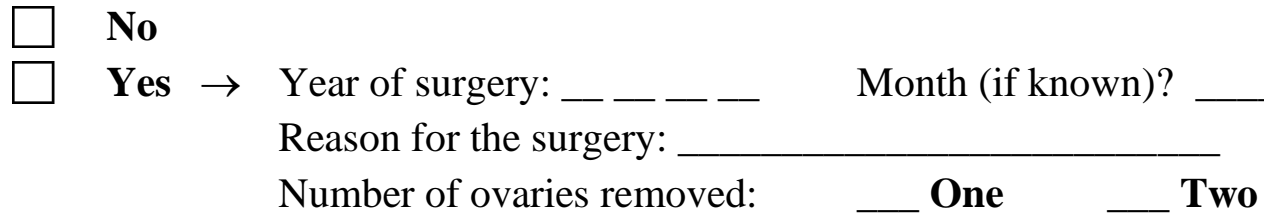

42. Have you ever had your fallopian tubes removed?

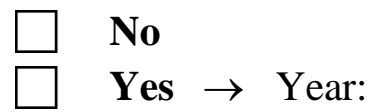

43. Have you ever had a tubal ligation (fallopian tubes tied)?

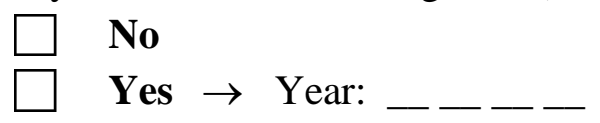

44. Have you had any other surgery performed on your reproductive organs: (e.g. hysterectomy, cervix removed, myomectomy, D \& C)

No

Yes $\rightarrow$ Year:

What operation was performed?

Reason for the surgery: 
45. Have you ever had ovarian cancer?

No $\rightarrow$ Go to question 51 (Section VI).

Yes $\rightarrow$ Year of diagnosis:

Month (if known)?

46. How was the cancer first detected?

Check one Screening (CA125, imaging) Symptoms presented (bloating, abdominal pain, etc.)

At the time of preventative surgery for ovarian cancer (removal of ovaries, fallopian tubes, etc.)

At the time of other abdominal surgery Other:

47. Did you have surgery for your ovarian cancer?

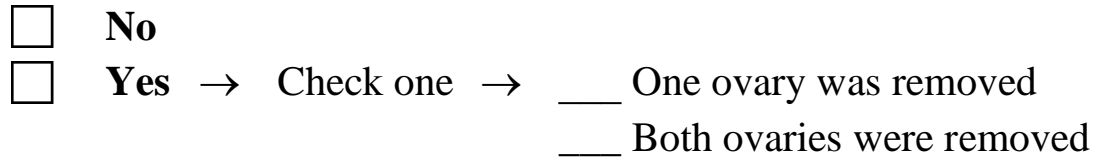

48. Did you receive chemotherapy for your ovarian cancer?

Yes $\rightarrow$ Was chemotherapy given before or after surgery?

Method of chemotherapy?

Check one $\rightarrow$ Intravenous (through the vein) Intraperitoneal (through the peritoneum) Both

49. Did you receive radiation therapy for your ovarian cancer?

No

Yes

50. Has your ovarian cancer come back or spread elsewhere (distant recurrence/metastasis)?

No

Yes $\rightarrow$ Date: (mm /yyyy)

Site of recurrence:

Treatment for recurrence: 


\section{SECTION VI - Personal Information}

Today's Date:

$$
\text { Month - Day - Year }
$$

Date of Birth:

$$
\text { Month - Year }
$$

Place of Birth:

Current Residence:

$$
\text { City Province/State }
$$

City Province/State

Ethnic Background:

51a. What is the major ancestry of your father? (Please circle one option.)

$\begin{array}{ll}\text { African or African American } & \text { Irish } \\ \text { (country of origin: _ } & \text { Italian } \\ \text { Ashkenazi Jewish } & \text { Native American (Amer. Indian) } \\ \text { Asian/Pacific Islander } & \text { Polish/Slavic/Eastern } \\ \quad \text { (country of origin: } & \text { Russian } \\ \text { Dutch } & \text { Scandinavian (Swedish/Finnish/ } \\ \text { English } & \text { Norwegian/Dane) } \\ \text { European Bloc countries } & \text { Scot-Irish or Scottish } \\ \text { French Canadian } & \text { Sephardic Jewish } \\ \text { German } & \text { Other (specify: } \\ \text { Hispanic } & \text { Unknown } \\ \quad \text { country of origin: } & \end{array}$

51b. What is the major ancestry of your mother? (Please circle one option.)

$\begin{array}{ll}\text { African or African American } & \text { Irish } \\ \text { (country of origin: } & \text { Italian } \\ \text { Ashkenazi Jewish } & \text { Native American (Amer. Indian) } \\ \text { Asian/Pacific Islander } & \text { Polish/Slavic/Eastern } \\ \quad \text { country of origin: } & \text { Russian } \\ \text { Dutch } & \text { Scandinavian (Swedish/Finnish/ } \\ \text { English } & \text { Norwegian/Dane) } \\ \text { European Bloc countries } & \text { Scot-Irish or Scottish } \\ \text { French Canadian } & \text { Sephardic Jewish } \\ \text { German } & \text { Other (specify: } \\ \text { Hispanic } & \text { Unknown } \\ \quad \text { country of origin: } & \end{array}$


52. What is your: current weight? pounds

current height? feet inches

Think back to when you were 18 years old, about the time you graduated from high school. How much did you weigh then? at age 30 ? pounds

at age 40 ? pounds pounds

What is the most you have ever weighed (exclude pregnancy)? pounds

How old were you when you weighed the most? years old

53. Do you know how much you weighed when you were born?

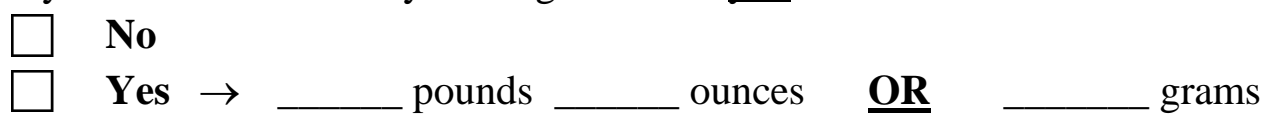

54. What is your mother's year of birth?

55. What is your birth order (i.e. first-born, second-born, third-born etc.)?

56. Were you part of a multiple birth (i.e. twin, triplet)?

$\square \quad$ No
$\square \quad$ Yes $\rightarrow$ (Please check one)

$\ldots$ twin $\rightarrow$ Are you an identical twin? $\square$ Yes $\square$ No triplet other (please specify):

\section{SECTION VII - Lifestyle}

57. Have you ever smoked cigarettes regularly?

$\begin{array}{ll}\square & \text { No } \\ \square & \text { Yes } \rightarrow \text { From: }\end{array}$ (age first started) to (age last used)

On average, how many packs do/did you smoke per week?

Do you still smoke?

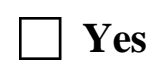

$\square$ No

58. Do you or did you ever drink coffee regularly?

$\begin{array}{ll}\square & \text { No } \\ \square & \text { Yes } \rightarrow \text { From: }\end{array}$

On average, how many cups do/did you drink a day?

Caffeinated: cups Decaffeinated: cups Total: cups Do you still drink coffee? Yes $\square$ No 
59. Do you drink alcoholic beverages?

$\begin{array}{ll}\square & \text { No } \\ \square \quad \text { Yes } \rightarrow \text { From: }\end{array}$ (age first started) to (age last used)

On average, how many alcoholic drinks do/did you have per week? (Please check one) 0-3 4-9 10-20 20 or more What type of alcoholic beverages do/did you drink? (Check all that apply) beer wine hard liquor

60. Have you ever been a regular user of talcum powder?

No

Yes $\rightarrow$ Did you apply it directly to the vaginal area?

Did you apply it to sanitary napkins?

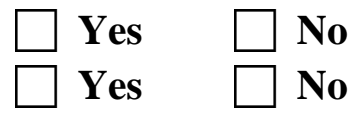

Other use of talcum powder? (describe)

What age did you start using talcum powder? years old

What age did you stop using talcum powder? years old

Do you still use talcum powder?

$\square$ Yes No

61. DURING THE PAST YEAR, what was your average time PER WEEK spent at each of the following recreational activities?

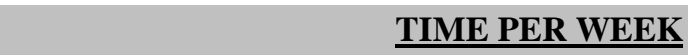

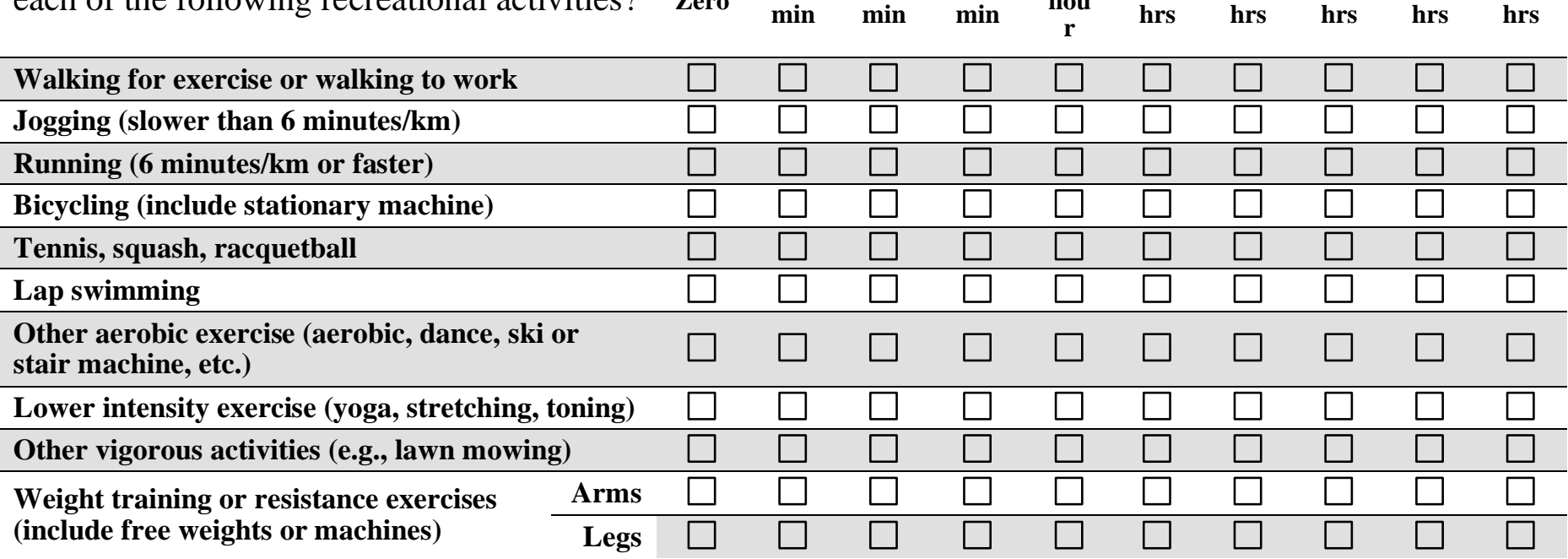

62. DURING THE PAST YEAR, on average, how many hours PER WEEK did you spend:

\section{TIME PER WEEK}

\begin{tabular}{lllllllllll}
\hline Standing or walking around work or away from home & $\square$ & $\square$ & $\square$ & $\square$ & $\square$ & $\square$ & $\square$ & $\square$ & $\square$ \\
\hline Standing or walking around at home & $\square$ & $\square$ & $\square$ & $\square$ & $\square$ & $\square$ & $\square$ & $\square$ & $\square$ \\
\hline Sitting at work or away from home or while driving & $\square$ & $\square$ & $\square$ & $\square$ & $\square$ & $\square$ & $\square$ & $\square$ \\
\hline Sitting at home while watching TV & $\square$ & $\square$ & $\square$ & $\square$ & $\square$ & $\square$ & $\square$ & $\square$ \\
\hline Other sitting at home (eg, reading, meal times, at desk) & $\square$ & $\square$ & $\square$ & $\square$ & $\square$ & $\square$ & $\square$ & $\square$ & $\square$
\end{tabular}




\section{SECTION VIII - Other Medical History}

63. Have you been diagnosed with any cancer other than breast or ovarian (not including a recurrence/metastasis of a breast or ovarian cancer)?

No

Yes $\rightarrow$ What type?

Year of Diagnosis?

64. Please describe briefly any medical problems that you have had in the past which are listed below. Check all that apply.

\begin{tabular}{|c|c|c|c|c|}
\hline Medical Condition & $\begin{array}{c}\text { Age of } \\
\text { Diagnosis }\end{array}$ & $\begin{array}{c}\text { Medication Taken for } \\
\text { Condition }\end{array}$ & $\begin{array}{l}\text { Medication } \\
\text { Start Year }\end{array}$ & $\begin{array}{c}\text { Medication } \\
\text { Stop Year }\end{array}$ \\
\hline$\square$ Osteoporosis & & & & \\
\hline $\begin{array}{l}\text { Thyroid Problems: } \\
\begin{array}{l}\square \text { Hyperthyroidism } \\
\square \text { Hypothyroidism }\end{array}\end{array}$ & & & & \\
\hline$\square$ High Cholesterol & & & & \\
\hline$\square$ High Blood Pressure & & & & \\
\hline 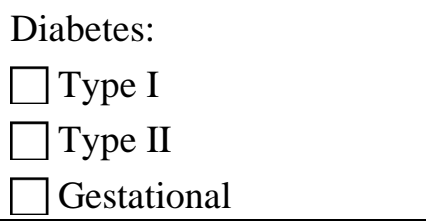 & & & & \\
\hline $\begin{array}{l}\text { Heart Disease: } \\
\square \text { Cardiomyopathy } \\
\square \text { Angina } \\
\square \text { Heart Attack }\end{array}$ & & & & \\
\hline$\square$ Blood Clot & & & & \\
\hline$\square$ Stroke & & & & \\
\hline $\begin{array}{l}\text { Crohn's } \\
\text { Disease/Ulcerative Colitis }\end{array}$ & & & & \\
\hline $\begin{array}{l}\text { Urinary/Genital Problems: } \\
\square \text { Urinary Tract Infections } \\
\square \text { Fibroids } \\
\square \text { Endometriosis }\end{array}$ & & & & \\
\hline $\begin{array}{l}\text { Mental illness: } \\
\square \text { Depression } \\
\square \text { Anxiety } \\
\square \text { Dementia }\end{array}$ & & & & \\
\hline$\square$ Other: & & & & \\
\hline
\end{tabular}


65a. Are you taking, or have you taken, any prescription medications on a regular basis which were NOT previously listed?
$\square$ No
$\square$ Yes $\rightarrow \quad$ Complete below:

1. Drug:

Frequency (days/week):

From: (year) to (year)

Reason:

2. Drug:

Frequency (days/week):

From: (year) to (year)

Reason:

3. Drug:

Frequency (days/week):

From: (year) to (year)

Reason:

4. Drug:

Frequency (days/week):

From: (year) to (year)

Reason:

5. Drug:

Frequency (days/week):

From: (year) to (year)

Reason:

65b. Are you taking, or have you taken, any over-the-counter medications (i.e. Aspirin, Tylenol, Advil, Aleve, etc.,) on a regular basis which were NOT previously listed?

No

Yes $\rightarrow$ Complete below:

1. Drug:

Frequency (days/week):

From: (year) to (year)

Reason:

2. Drug:

Frequency (days/week):

From: (year) to (year)

Reason:

3. Drug:

Frequency (days/week):

From: (year) to (year)

Reason:

4. Drug:

Frequency (days/week):

From: (year) to (year)

Reason:

5. Drug:

Frequency (days/week):

From: (year) to (year)

Reason: 
66. Do you take dietary supplements or vitamins (i.e. multi-vitamins, calcium supplement etc.)?

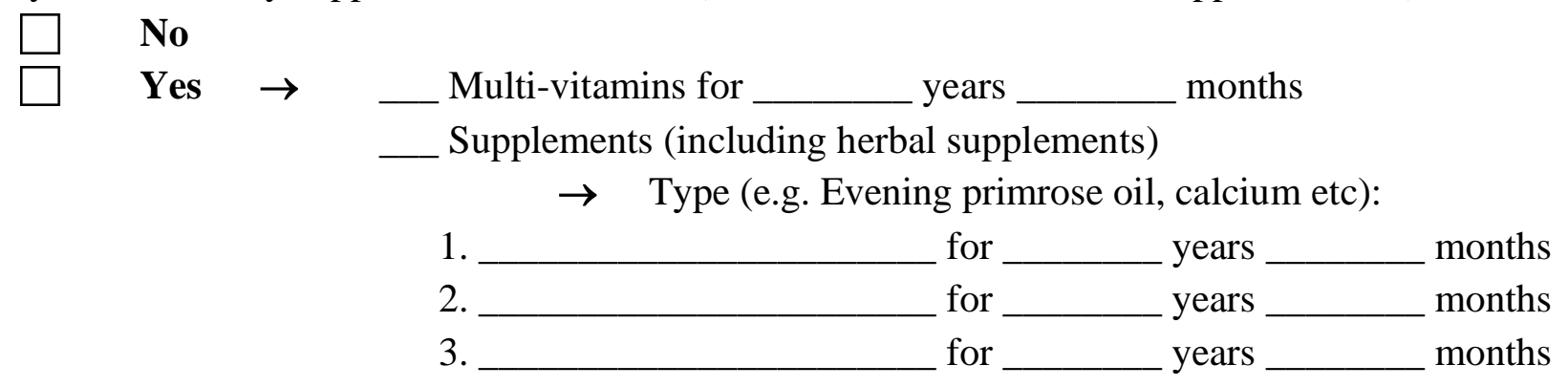

67. Have you ever had another operation on your abdomen? (e.g. gall bladder, appendix, hernia, etc)

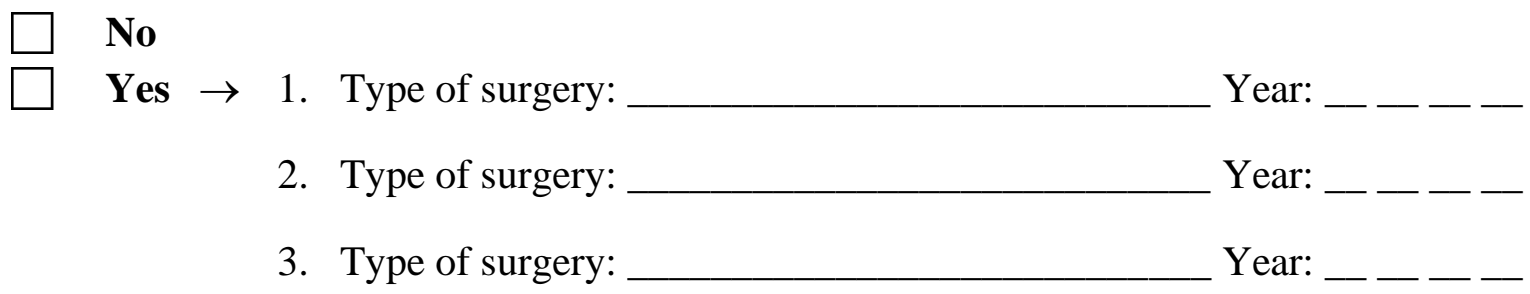

68. Have you received your genetic test results?

$\begin{array}{ll}\square & \text { No } \\ \square & \text { Yes } \rightarrow \text { Date you received your results: }\end{array}$ ' _ _ _ _ _ _ (mm/ dd / yyyy $)$ 
FOR OFFICE USE (to be completed by Genetic Counsellor):

Participating centre:

Contact person:

Interviewer (if by phone):

Family Number:

Individual Identification No:

Date questionnaire sent:

$$
\overline{\text { Month - Day - Year }}
$$

Date questionnaire received:

$$
\text { Month - Day - Year }
$$

Information received by mail or by telephone or in clinic

Genetic Test Result Disclosed to Patient? $\square$ Yes $\square$ No

Date Test Result Disclosed:

Mutation: BRCA_:

PALB2 : Exon 
Appendix 2. Follow-up Questionnaire for the Risk Factor Analysis of Breast and Ovarian Cancer

\author{
FOLLOW-UP QUESTIONNAIRE FOR
}

\title{
A STUDY OF BREAST AND OVARIAN CANCER \\ IN HIGH RISK FAMILIES
}

Date Completed:

(mm / dd / yyyy)

Name:

Date of Birth: (mm / dd / уyyy)

Fam/Ind\#:

Telephone \#:

Date of Previous Questionnaire: (mm / dd / уyyy)

Centre: 


\section{SECTION I - Reproductive Update}

1. Have you had any pregnancies since I (mm / yyyy) (including still born, miscarriage, abortion)?

No $\rightarrow$ Go to question 4 .

Yes $\rightarrow$ Please consider any NEW pregnancies since the date above in order from first to last. Give year of pregnancy. Indicate the outcome of each pregnancy. For liveborn children, try to recall how long you breast-fed each child and indicate months of breast-feeding (if not breast-fed, enter ' 0 ').

\begin{tabular}{|l|c|c|c|c|c|c|c|}
\hline \# & \multicolumn{2}{|c|}{ PREGNANCY } \\
$\begin{array}{c}\text { Year of } \\
\text { Pregnancy }\end{array}$ & $\begin{array}{c}\text { Length of } \\
\text { Pregnancy (In } \\
\text { Weeks) }\end{array}$ & $\begin{array}{c}\text { Pregnancy Outcome } \\
\text { (Live, Still, } \\
\text { Miscarriage, } \\
\text { Abortion, Twins, } \\
\text { etc.) }\end{array}$ & $\begin{array}{c}\text { Live Birth } \\
\text { Caesarean } \\
\text { Section? (Yes, } \\
\text { No) }\end{array}$ & $\begin{array}{c}\text { Live Born } \\
\text { (Month/Year of } \\
\text { Birth) }\end{array}$ & $\begin{array}{c}\text { Birth } \\
\text { Weight } \\
\text { (lbs.) }\end{array}$ & $\begin{array}{c}\text { Months } \\
\text { of } \\
\text { Breast- } \\
\text { Feeding }\end{array}$ \\
\hline 1 & & & & & & & \\
\hline 2 & & & & & & & \\
\hline
\end{tabular}

2. Did you ever have difficulty breast-feeding?

$\begin{array}{ll}\square & \text { No } \\ \square & \text { Yes } \rightarrow \text { Reason: }\end{array}$

_ premature infant _ other (please specify):
_ pain

_ mastitis (breast inflammation)

3. Have you ever taken medication to stop milk production?

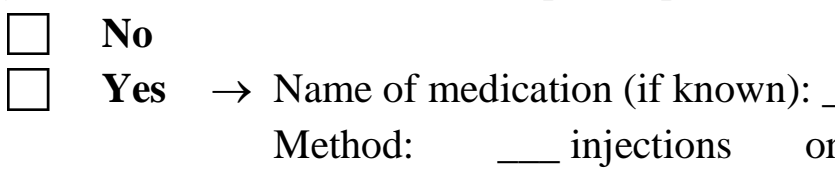

or pills

4. Are you still having menstrual periods? That is, have you had a menstrual period within the last year? Please answer "Yes" if you are currently pregnant, breast-feeding or taking hormones which temporarily stop your menses (Mirena IUD or birth control pills.)

$\square \quad$ Yes

No $\rightarrow$ At what age did they stop?

Reason they stopped? (Please check only one)

$\square \quad$ Natural Menopause (change of life)

$\square$ Hysterectomy (uterus removed only, ovaries not removed)

One ovary removed only

$\square$ Both ovaries removed only

$\square$ Fallopian tubes and ovaries removed 
$\square$ Uterus, fallopian tubes, and ovaries removed

Medication / Chemotherapy

Other (please specify):

5. Have you ever taken birth control medication including birth control pills, Norplant (implants), Mirena/IUD, or Depo-Provera (injections) to prevent pregnancy or for any other reason?

No

Yes $\rightarrow$ Please complete the table below:

\begin{tabular}{|c|l|l|l|l|l|}
\hline \multicolumn{1}{|c|}{ Method } & Name of Medication & $\begin{array}{c}\text { Starting } \\
\text { Year }\end{array}$ & $\begin{array}{c}\text { Ending } \\
\text { Year }\end{array}$ & $\begin{array}{c}\text { Length of Time } \\
\text { Used in Years }\end{array}$ \\
\hline 1 & $\begin{array}{l}\square \text { Pills } \square \text { Implants } \\
\square \text { Mirena/IUD } \\
\square \text { Injections } \square \text { Other }\end{array}$ & & & \\
\hline 2 & $\begin{array}{l}\square \text { Pills } \square \text { Implants } \\
\square \text { Mirena/IUD } \\
\square \text { Injections } \square \text { Other }\end{array}$ & & & \\
\hline
\end{tabular}

\section{Are you still using birth control medication? $\square$ Yes $\square$ No}

6. Have you ever taken hormone replacement therapy (HRT)?

No

Yes $\rightarrow$ Please complete the table below:

\begin{tabular}{|c|c|c|c|c|c|}
\hline & Method & $\begin{array}{c}\text { Name of } \\
\text { Medication }\end{array}$ & $\begin{array}{c}\text { Starting } \\
\text { Year }\end{array}$ & $\begin{array}{c}\text { Ending } \\
\text { Year }\end{array}$ & $\begin{array}{c}\text { Length of Time } \\
\text { Used in Years }\end{array}$ \\
\hline 1 & $\begin{array}{l}\square \text { Pills } \square \text { Skin Patches } \\
\square \text { Vaginal Suppositories } \\
\square \text { Gel } \square \text { Cream } \\
\square \text { Ring } \square \text { Injections } \\
\square \text { Other }\end{array}$ & & & & \\
\hline 2 & $\begin{array}{l}\square \text { Pills } \square \text { Skin Patches } \\
\square \text { Vaginal Suppositories } \\
\square \text { Gel } \square \text { Cream } \\
\square \text { Ring } \square \text { Injections } \\
\square \text { Other }\end{array}$ & & & & \\
\hline
\end{tabular}

Are you still using HRT? $\square$ Yes $\square$ No 


\section{SECTION II - Lifestyle}

7. Have you ever smoked cigarettes?

No
Yes $\rightarrow$ From: (age first started) to (age last used)

On average, how many packs do/did you smoke per week?
Do you still smoke?
Yes
No

8. Have you ever drank coffee?
No
Yes $\rightarrow$ From: (age first started) to (age last used)
On average, how many cups do/did you drink a day?
Caffeinated: cups Decaffeinated: cups Total: cups

\section{Do you still drink coffee? $\square$ Yes $\square$ No}

9. Have you ever drank alcohol?
$\begin{array}{lll} & \text { No } \\ \square & \text { Yes } \rightarrow & \text { From: }\end{array}$ (age first started) to (age last used)
On average, how many alcoholic drinks do/did you have per week? (Check one)

$$
0-3 \quad 4-9 \quad 10-20 \_20 \text { or more }
$$
What type of alcoholic beverages do/did you drink? (Check all that apply) beer wine hard liquor
Do you still drink alcohol? $\square$ Yes $\square$ No

10. What is your current weight: pounds

$\underline{\text { or }}$ kgs

11. What is your highest level of education? (please check one)

No Schooling Attended elementary school Attended high school Attended college/university Attended graduate school Graduated from elementary school Graduated from high school Graduated from college/university Graduated from graduate school

12. DURING THE PAST YEAR, what was your average time PER WEEK spent at each of the following recreational activities?

\section{TIME PER WEEK}

\begin{tabular}{lllllllllll}
\hline Walking for exercise or walking to work & $\square$ & $\square$ & $\square$ & $\square$ & $\square$ & $\square$ & $\square$ & $\square$ & $\square$ & $\square$ \\
\hline Jogging (slower than 6 minutes/km) & $\square$ & $\square$ & $\square$ & $\square$ & $\square$ & $\square$ & $\square$ & $\square$ & $\square$ & $\square$ \\
\hline Running (6 minutes/km or faster) & $\square$ & $\square$ & $\square$ & $\square$ & $\square$ & $\square$ & $\square$ & $\square$ & $\square$ & $\square$ \\
\hline Bicycling (include stationary machine) & $\square$ & $\square$ & $\square$ & $\square$ & $\square$ & $\square$ & $\square$ & $\square$ & $\square$ & $\square$ \\
\hline Tennis, squash, racquetball & $\square$ & $\square$ & $\square$ & $\square$ & $\square$ & $\square$ & $\square$ & $\square$ & $\square$ & $\square$ \\
\hline Lap swimming & $\square$ & $\square$ & $\square$ & $\square$ & $\square$ & $\square$ & $\square$ & $\square$ & $\square$ & $\square$ \\
\hline
\end{tabular}




\begin{tabular}{lllllllllll}
$\begin{array}{l}\text { Other aerobic exercise (aerobic, dance, ski or } \\
\text { stair machine, etc.) }\end{array}$ & $\square$ & $\square$ & $\square$ & $\square$ & $\square$ & $\square$ & $\square$ & $\square$ & $\square$ \\
\hline Lower intensity exercise (yoga, stretching, toning) & $\square$ & $\square$ & $\square$ & $\square$ & $\square$ & $\square$ & $\square$ & $\square$ & $\square$ \\
\hline Other vigorous activities (e.g., lawn mowing) & $\square$ & $\square$ & $\square$ & $\square$ & $\square$ & $\square$ & $\square$ & $\square$ & $\square$ \\
\hline $\begin{array}{l}\text { Weight training or resistance exercises } \\
\text { (include free weights or machines) }\end{array}$ & Arms & $\square$ & $\square$ & $\square$ & $\square$ & $\square$ & $\square$ & $\square$ & $\square$ & $\square$ \\
\cline { 2 - 8 }
\end{tabular}

13. DURING THE PAST YEAR, on average, how many hours PER WEEK did you spend:

\section{TIME PER WEEK}

\begin{tabular}{llllllllll}
\hline Standing or walking around work or away from home & $\square$ & $\square$ & $\square$ & $\square$ & $\square$ & $\square$ & $\square$ & $\square$ \\
\hline Standing or walking around at home & $\square$ & $\square$ & $\square$ & $\square$ & $\square$ & $\square$ & $\square$ & $\square$ & $\square$ \\
\hline Sitting at work or away from home or while driving & $\square$ & $\square$ & $\square$ & $\square$ & $\square$ & $\square$ & $\square$ & $\square$ \\
\hline Sitting at home while watching TV & $\square$ & $\square$ & $\square$ & $\square$ & $\square$ & $\square$ & $\square$ & $\square$ & $\square$ \\
\hline Other sitting at home (eg, reading, meal times, at desk) & $\square$ & $\square$ & $\square$ & $\square$ & $\square$ & $\square$ & $\square$ & $\square$ & $\square$
\end{tabular}

\section{SECTION III - Breast Cancer Screening/Prevention}

14. Have you ever taken any drugs for the prevention of breast cancer (i.e. drugs taken before any diagnosis of breast cancer)?

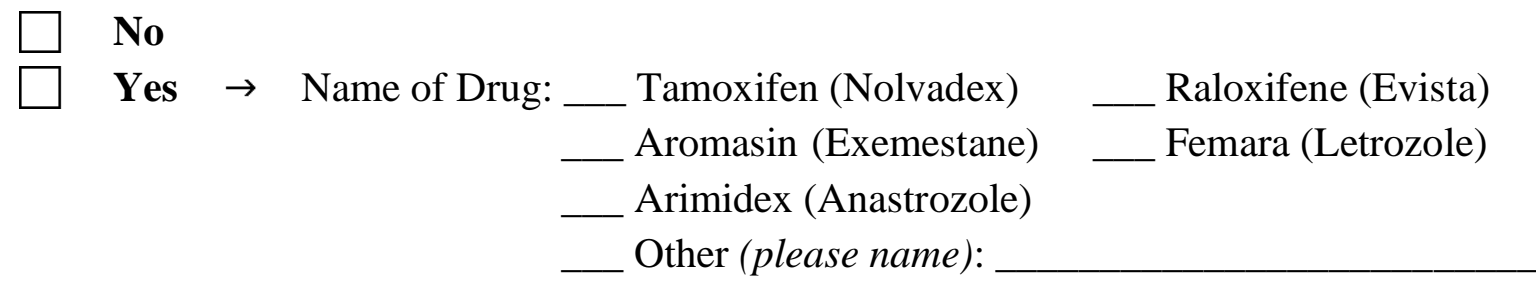

Dosage (mg/day):

Date started: ___ _ _ _ _ (mm/yyyy) to _ _

15. Have you ever had a mammogram?

No

Yes $\rightarrow$ Age at first mammogram:

How many mammograms have you had?

Were there any abnormalities detected by mammogram? $\square$ Yes $\square$ No

If yes, please describe:

Month/Year of abnormality: I

What year was your last mammogram done? 
16. Have you ever had MRI screening of your breasts?

$\square \quad$ No
$\square \quad$ Yes $\rightarrow \quad$ Age at first MRI:

Month/Year of first MRI:

How many MRIs have you had?

Where were they done:

Were there any abnormalities detected by MRI?

Yes

No

If yes, please describe:

Year of abnormality:

Month/year of your last MRI?

17. Have you ever had a breast biopsy (this includes needle and core biopsies)?

$\square \quad$ No

Yes $\rightarrow$ Number of biopsies:

Date of first biopsy:

Date of last biopsy:

_ _ _ _ _ _ (

_ _ _ _ _ _ (mm / ууyу $)$

What was the result of the biopsy? (Check all that apply)

$\begin{array}{lll}\text { normal } & \text { DCIS } & \text { D atypical hyperplasia } \\ \text { cancer } & \text { LCIS } & \text { - fibroadenoma } \\ \text { other (please specify) } & \end{array}$

18. Have you undergone preventive removal of your breasts? NOTE: The surgery is only preventive if you've never previously been diagnosed with cancer in that breast.

No

Yes $\rightarrow$ Date:

I__ _ _ _ _ _ ( $(\mathrm{mm} / \mathrm{dd} /$ yyyy $)$

Which breast was removed?

Left

Right

Both

Procedure: __ Subcutaneous Mastectomy (nipple and areola preserved) Total Mastectomy (nipple and areola removed) Other:

19. Have you ever had breast implants or breast reconstruction?

$\square \quad$ No
$\square \quad$ Yes $\rightarrow$ Type: $\_$Saline
Year of surgery: $\_\_$
Other
Have you ever had breast reduction?
$\square \quad$ No
$\square \quad$ Yes $\rightarrow \quad$ Year of surgery: $\_\ldots \ldots$




\section{SECTION IV - Breast Cancer (Previous Diagnosis)}

Did you have breast cancer at the time of or prior to the date of the last questionnaire?

NO $\rightarrow$ Go to Question 24 (Section V).

YES $\rightarrow$ Please complete this section

Year of previous cancer: $\ldots \ldots$ __ _ _ $(\mathrm{mm} /$ yyyy $)$

Side: __ Left _ Right _ Both

Type (check all that apply):

_ Invasive

$\ldots$ Non-invasive $\rightarrow$ _ D Ductal Carcinoma In-situ (DCIS)

_ Lobular Carcinoma In-situ (LCIS)

Treatment: Surgery: Radiation Chemotherapy Hormone Therapy

21. Have you ever taken any drugs for the treatment (and to prevent a recurrence) of your breast cancer?

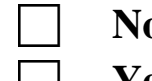

Yes $\rightarrow$ Name of Drug: Tamoxifen (Nolvadex) Raloxifene (Evista) Aromasin (Exemestane) Femara (Letrozole) Arimidex (Anastrozole) Other (please name):

Dosage (mg/day):

Date started:

' _ _ _ (mm/yyyy) to (mm /yyyy)

22. Has your breast cancer come back in the same breast (local recurrence) or spread elsewhere (distant recurrence/metastasis)?

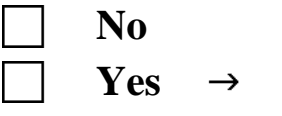

Site: (mm / dd / yyyy)

Mode of detection: $\begin{aligned} & \text { Mammogram } \\ & \text { Self-exam }\end{aligned}$ _ Other: _ _ _ _ Doctor

Treatment:

23. Have you had any further surgery on the affected breast(s)?

$\square \quad$ No

$\square$ Yes $\rightarrow$ Type of surgery:

Lumpectomy (part of breast removed)
Which breast? _ Left _
Date of lumpectomy:

Mastectomy (whole breast removed)

Which breast? __ Left _ Right _ Both 
Date of mastectomy: I _ ${ }^{\prime}+\ldots \ldots(\mathrm{mm} / \mathrm{dd} / \mathrm{yyyy})$

\section{Reconstruction}

Which breast? _ Left

_ Right _ Both ${ }^{\text {B }}$ ___ $/$ dd/yyy $)$

\section{SECTION V - Ovarian Cancer Screening/Prevention}

24. Have you ever had a trans-vaginal ultrasound (internal ultrasound done through the vagina)?

$\square$ No

Yes $\rightarrow$ Age at first ultrasound:

Number of ultrasounds:

Abnormalities detected by ultrasound: $\square$ Yes $\square$ No

If Yes: Describe:

Year of abnormality:

Year of last trans-vaginal ultrasound:

25. Have you ever had a blood test for CA125 (marker for ovarian cancer)?

No

Yes $\rightarrow$ Age at first CA125:

Number of CA125 tests:

Abnormalities detected:

No Elevated

Don't Know

Year of abnormality:

Year of the last CA125:

26. Have you had your ovaries removed (oophorectomy) for reasons other than ovarian cancer? (e.g. preventative measures, fibroids, cyst, scar tissue, or pain)

No

Yes $\rightarrow$ Number of ovaries removed:

Year of surgery:

If ovaries removed in separate surgeries:

Month/Year of second surgery

Reason: Abnormal CA125 test Preventative Cyst Other

27. Have you ever had your fallopian tubes removed?

$\square \quad$ No
$\square \quad$ Yes $\rightarrow$ Year:

28. Have you ever had a tubal ligation (fallopian tubes tied)?

$\square \quad$ No
$\square \quad$ Yes $\rightarrow$ Year: 
29. Have you had any other surgery performed on your reproductive organs: (e.g. hysterectomy, cervix removed, myomectomy, D \& C)

$\begin{array}{ll}\square & \text { No } \\ \square \quad \text { Yes } \rightarrow \text { Year: }\end{array}$

What operation was performed?

Reason for the surgery:

\section{SECTION VI - Ovarian Cancer (Previous Diagnosis)}

Did you have ovarian cancer at the time of or prior to the date of the last questionnaire?

$\square \quad$ NO $\rightarrow$ Go to Question 32 (Section VII).
YES $\rightarrow$ Please complete this section
Year of previous cancer: ________ $(\mathrm{mm} /$ yyyy $)$

30. Has your ovarian cancer come back or spread elsewhere (distant recurrence/metastasis)?

No

Yes $\rightarrow$ Date: ___ _ _

Site:

Mode of detection: __ CA125 test _ _ Trans-vaginal ultrasound Doctor _ Other

Treatment:

31. Do you still have your ovaries?

No

Yes $\rightarrow$ How many?

$\square \quad \square \quad \square \quad 2$

\section{SECTION VII - New Breast Cancer Diagnosis}

32. Have you been diagnosed with breast cancer since __ _ _ _ _ _ $(\mathrm{mm} /$ yyyy $)$ ?

No $\rightarrow$ Go to Question 34 (Section VIII).

Yes $\rightarrow$ Date of diagnosis: _ _ $/ \ldots \ldots$ _ _ _ _ $(\mathrm{mm} / \mathrm{dd} /$ yyyy $)$

Which breast? __ Left __ Right _ _ Both

\section{Type of Cancer (check all that apply):}

$\square \quad$ It was an invasive cancer.

$\square \quad$ It was a non-invasive cancer (early stage breast cancer).

$\rightarrow$ Was it Ductal Carcinoma In-situ (DCIS)? $\square$ Yes $\square$ No

$\rightarrow$ Was it Lobular Carinoma In-situ (LCIS)? $\square$ Yes $\square$ No

$\square \quad$ I'm not sure. 
Treatment: (i) Surgery? $\square$ Yes $\square$ No

If Yes: __ Mastectomy (whole breast removed)

Which breast? _ Left _ Right _ Both

Date of mastectomy: ___ _ _ _ _ _ _ $(\mathrm{mm} / \mathrm{dd} / \mathrm{yyyy})$

Lumpectomy (part of breast removed)

Which breast? _ Left _ Right _ Both

Date of lumpectomy: _ _

Axillary Node Dissection (lymph nodes removed)

Number removed:

Were any found to have cancer? $\square$ Yes $\square$ No

How many?

(ii) Chemotherapy? $\square$ Yes $\square$ No

If yes, name of drug:

(iii) Radiation therapy? $\square$ Yes $\square$ No

(iv) Other treatment drugs (e.g. Tamoxifen etc.)? $\square$ Yes $\square$ No

If yes, name of drug:

Dosage (mg/day):

Date started:

to

I____ (mm/yyyy)

Name of hospital:

City:

How was the cancer first detected?

If a lump was felt, was a mammogram done to confirm the cancer?

Yes $\square$ No

If yes, was an abnormality detected?

Yes $\square$ No

33. Has your breast cancer come back in the same breast (local recurrence) or spread elsewhere (distant recurrence/metastasis)?

No

Yes $\rightarrow$ Date: __l__

Site of recurrence:

Treatment: 


\section{SECTION VIII - New Ovarian Cancer Diagnosis}

34. Have you been diagnosed with ovarian cancer since (mm / ууyу)?

No $\rightarrow$ Go to Question 36 (Section IX).

Yes $\rightarrow$ Date of diagnosis: ' _ _ _ _ _ $(\mathrm{mm} / \mathrm{dd} /$ уууу $)$

Treatment: (i) Ovaries removed? $\square$ Yes $\square$ No

If Yes: How many? 12

Date of surgery: _ _

(ii) Chemotherapy?

Yes $\square$ No

If Yes: Name of drug:

(iii) Radiation therapy?

Yes

No

Name of hospital:

City:

How was the cancer first detected?

Was a trans-vaginal ultrasound done?

$\begin{array}{ll}\square \text { Yes } & \square \text { No } \\ \square \text { Yes } & \square \text { No } \\ \square \text { Yes } & \square \text { No } \\ \square \text { Yes } & \square \text { No }\end{array}$

If yes, were any abnormalities detected? Was a CA125 test done?

If yes, were any abnormalities detected?

35. Has your ovarian cancer come back or spread elsewhere (distant recurrence/metastasis)?

No

Yes $\rightarrow$ Date: (mm / dd / yyyy)

Site of recurrence:

Treatment:

\section{SECTION IX - Other Medical History}

36. Have you been diagnosed with any cancer other than breast or ovarian (not including a recurrence/metastasis of a breast or ovarian cancer)?

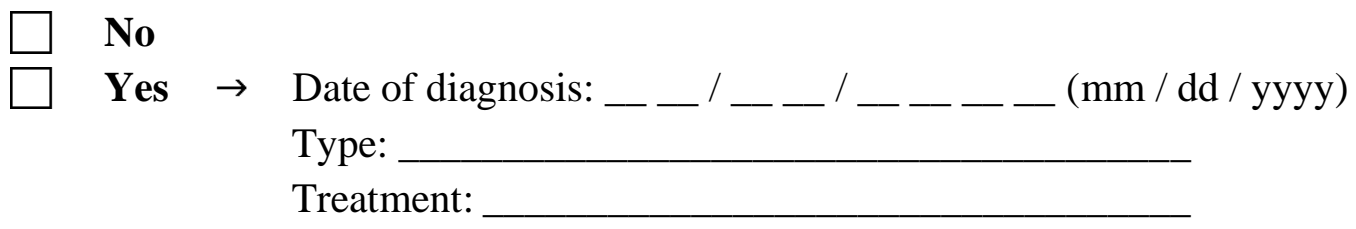


37. Please describe briefly any other medical problems that you have had in the past which are listed below. Check all that apply.

\begin{tabular}{|l|l|l|l|l|}
\hline \multicolumn{1}{|c|}{ Medical Condition } & $\begin{array}{c}\text { Age of } \\
\text { Diagnosis }\end{array}$ & $\begin{array}{c}\text { Medication Taken for } \\
\text { Condition }\end{array}$ & $\begin{array}{c}\text { Medication } \\
\text { Start Year }\end{array}$ & $\begin{array}{c}\text { Medication } \\
\text { Stop Year }\end{array}$ \\
\hline$\square$ Osteoporosis & & & & \\
\hline $\begin{array}{l}\text { Thyroid Problems: } \\
\square \text { Hyperthyroidism }\end{array}$ & & & & \\
\hline$\square$ High Cholesterol & & & & \\
\hline $\begin{array}{l}\text { High Blood Pressure } \\
\text { Diabetes: }\end{array}$ & & & & \\
$\square$ Type I & & & & \\
$\square$ Type II & & & \\
$\square$ Gestational & & & & \\
\hline Heart Disease: & & & \\
$\square$ Cardiomyopathy & & & & \\
$\square$ Angina & & & & \\
$\square$ Heart Attack & & & & \\
\hline$\square$ Blood Clot & & & & \\
\hline$\square$ Stroke & & & & \\
\hline$\square$ Crohn's & & & & \\
Disease/Ulcerative Colitis & & & & \\
\hline Urinary/Genital Problems: \\
$\square$ Urinary Tract Infections \\
$\square$ Fibroids \\
$\square$ Endometriosis
\end{tabular}


38a. Have you taken any other prescription medications on a regular basis since I - - - (mm/yyyy) which were NOT previously mentioned?

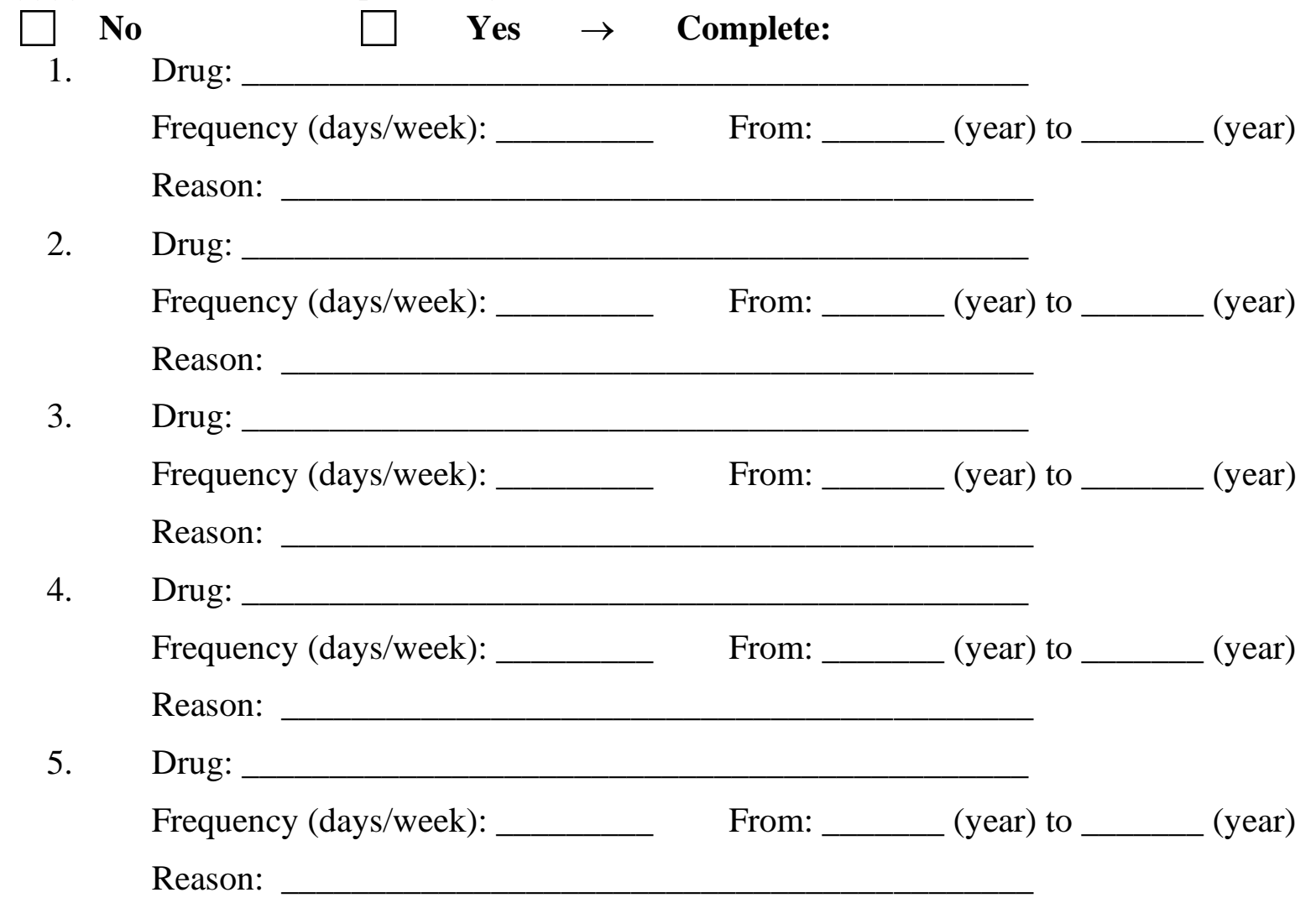

38b. Have you taken any new over-the-counter medications (i.e. Aspirin, Tylenol, Advil, Aleve, etc.,) on a regular basis since ___ _ _ _ _ _ ( $\mathrm{mm} /$ yyyy $)$ which were NOT previously mentioned?

$\square$ No $\quad \square$ Yes $\rightarrow \quad$ Complete:

1. Drug:

Frequency (days/week):

From: (year) to (year)

Reason:

2. Drug:

Frequency (days/week):

From: (year) to (year)

Reason:

3. Drug:

Frequency (days/week):

From: (year) to (year)

Reason:

4. Drug:

Frequency (days/week):

From: (year) to (year) 


\section{SECTION XII - PEDIGREE UPDATE}

42. Have there been any new cancers diagnosed in your family since I__ _ _ (mm / уууу $) ?$

$\square \quad$ No

$\square \quad$ Yes (Complete table below)

\begin{tabular}{|l|l|l|l|}
\hline Name & & & \\
\hline Relationship & & & \\
\hline Type of Cancer & & & \\
\hline Year of Diagnosis & & & \\
\hline Age at Diagnosis & & & \\
\hline
\end{tabular}

43. Have there been any births or deaths in your family since _ _ _ _ _ _ _ (

$\begin{array}{lll}\square & \text { No } \\ \square \quad \text { Yes } \quad \text { (Complete table below) }\end{array}$

\begin{tabular}{|l|l|l|l|}
\hline Name & & & \\
\hline Relationship & & & \\
\hline Date of Birth & & & \\
\hline Date of Death & & & \\
\hline Cause of Death & & & \\
\hline
\end{tabular}


Thank you very much for taking the time to complete this questionnaire. Should the need arise, may we contact you again? $\square$ No $\square$ Yes

Telephone number:

Alternate contact:

E-mail address:

Mailing address:

CITY:

PROVINCE:

Postal Code: 


\title{
Appendix 3. Informed Consent Form for the Risk Factor Analysis of Breast and Ovarian Cancer
}

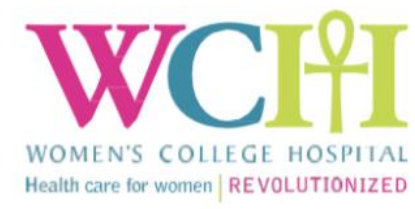

\author{
Dr. Steven Narod, MD, FRCPC \\ Canada Research Chair in Breast Cancer \\ 76 Grenville Street, 6 th Floor, Toronto, Ontario MSS1B2 \\ Tel: 416-351-3765 Fax: 416-351-3767 E-mail: steven.narod@wchospital.ca
}

\section{INFORMED CONSENT FORM}

Risk Factor Analysis of Hereditary Breast and Ovarian Cancer

\author{
PRINCIPAL INVESTIGATOR: Dr. Steven Narod \\ SITE: \\ SPONSOR: \\ Women's College Research Institute \\ The Prevention Research Grant of the Canadian Cancer Society and the Canadian \\ Institutes of Health Research-Institute of Cancer Research
}

We would like to invite you to take part in a multi-site research study called the Risk Factor Analysis of Hereditary Breast and Ovarian Cancer.

\section{BACKGROUND:}

Women with a $B R C A 1 / 2$ mutation have a high lifetime risk of developing breast cancer $(80 \%$ vs. $12 \%$ for women without the mutation) and /or ovarian cancer (30\% vs. $3.5 \%$ ). Women who have a PALB2 mutation can also participate in this study. Prevention in these women at high risk is an important part of their care. This project began in 1996 studying women with any of these mutations. As of 2014, this study has upwards of 15,000 participants from around the world.

\section{PURPOSE OF RESEARCH:}

The purpose of this study is to identify hormonal, reproductive and lifestyle factors that are associated with the risk of developing breast and ovarian cancer among women at high risk. It is expected that around 20,000 individuals will participate in this project.

\section{WHO CAN PARTICIPATE IN THE STUDY?}

- Individuals who fully understand the study and give informed consent to participate as demonstrated by signing the provided consent form.

- Women with BRCA1 and/or BRCA2 and/or PALB2 mutations.

- Women who are at least 18 years of age.

\section{WHO CANNOT PARTICIPATE IN THE STUDY?}

- Women who do not meet the criteria above.

- Individuals who are unable to give voluntary, informed consent.

\section{PROCEDURE:}

You will be asked to complete a baseline questionnaire asking about your medical history, family history of cancer and factors related to your lifestyle. You will be asked to update us every two years by completing a new questionnaire. As a result, members of the research team may contact you by telephone or by mail every two years to obtain follow-up questionnaires as part of this research study. Providing correct information about your medical history is your responsibility. You authorize members of the research team to access your pathology and medical records for research purposes. In the event that additional health information is required by the research team and you no longer have the capacity to provide health information, the research team has consent to contact your next of kin to obtain the information.

Participants will be asked to contribute to the research for a maximum of twenty years. The questionnaire data, in an anonymous form (a form that does not identify you), may be sent to other academic institutions for additional studies on the hereditary basis of cancer. Data from the questionnaire will not be used for purposes other than the study of familial cancer. 

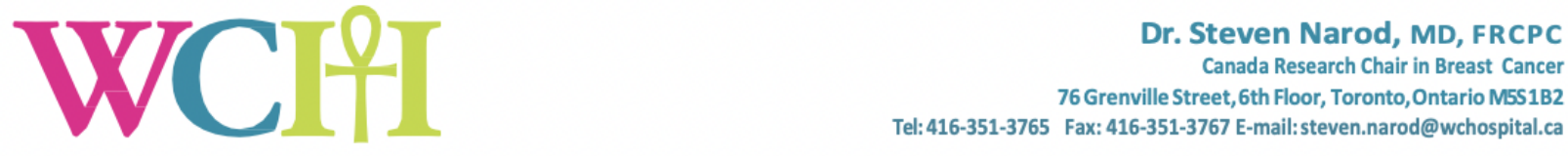

Health care for women REVOLUTIONIZED

\section{CONFIDENTIALITY:}

You understand that medical and all other information produced by this study will be strictly confidential and will be held securely at and will be subject to the confidentiality and privacy regulations of Women's College Hospital. This information will not become part of your personal medical record. This information will be available to the study research team, and not released to any other party, except upon your expressed written consent. Information regarding your medical history or test results will not be disclosed to any other member of your family. Information pertaining to the medical history or test results of your relatives will not be disclosed to you.

You have the right to have any information about you and your health that is collected, used or disclosed for this study to be handled in a confidential manner.

If you decide to participate in this study, the investigator(s) and study staff will look at your personal health information and collect only the information they need for this study. It includes information such as your

- name,

- address,

- telephone number,

- date of birth,

- new and existing medical records, or

- the types, dates and results of various tests and procedures.

You have the right to access, review and request changes to your personal health information.

\section{BENEFITS:}

You may or may not benefit directly from participating in the study. Genetic counselling will be available to you and members of your family. The research team will be available to provide the most current information regarding genetic risk assessment and will provide referral to screening centres for cancer if requested. You understand that you may have access to any information regarding your personal risk of developing cancer upon request. You understand that there is no compensation available for your participation in this research study. You understand that representatives of the National Cancer Institute of Canada, (the research granting agency) may inspect the research records.

\section{RISKS:}

There are no medical risks to you from participating in this study but taking part in this study may make you feel uncomfortable or feel distressed answering some of the questions in the questionnaire. If you do not feel comfortable responding to a question, you may feel free to skip it. If you require additional support, please talk to the investigator who will discuss additional support options with you.

\section{WITHDRAWAL FROM STUDY PARTICIPATION}

You understand that your participation is voluntary and if you agree to participate you may withdraw your consent and discontinue your participation at any time without prejudice to or loss of your medical care or the benefits to which you, or your family, are otherwise entitled. If you withdraw your consent, the information about you that was/were collected before you left the study will still be used. No new information about you will be collected (and no further testing of you will be done) without your permission. You understand that your participation will not affect your choice of, or access to, treatment or screening. You understand that your participation may be terminated with or without your consent. 


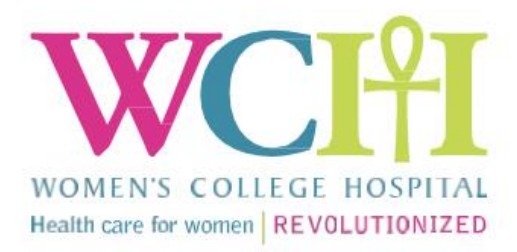

Dr. Steven Narod, MD, FRCPC

Canada Research Chair in Breast Cancer 76 Grenville Street, 6th Floor, Toronto, Ontario M5S1B2

Tel: 416-351-3765 Fax: 416-351-3767 E-mail: steven.narod@wchospital.ca

\section{REQUEST FOR MORE INFORMATION:}

You understand that you may ask more questions about the study. Dr. Narod is available to answer your questions and concerns (Tel. (1)-416-351-3765). If you have any questions about your rights as a research participant, please call Melissa Sidhu, Women's College Research Ethics Board Manager, at (416) 351-3732 Ext 2723. This person is not involved with the research project in any way and calling her will not affect your participation in the study.

\section{CONFIRMATION OF PARTICIPATION:}

\section{DOCUMENTATION OF INFORMED CONSENT}

You will be given a copy of this informed consent form after it has been signed and dated by you and the study staff.

Full Study Title: Risk Factor Analysis of Hereditary Breast and Ovarian Cancer

Name of Participant:

Participant/Substitute decision-maker

By signing this form, I confirm that:

- This research study has been fully explained to me and all of my questions answered to my satisfaction

- I understand the requirements of participating in this research study

- I have been informed of the risks and benefits, if any, of participating in this research study

- I have been informed of any alternatives to participating in this research study

- I have been informed of the rights of research participants

- I have read each page of this form

- I authorize access to my personal health information, medical record and research study data as explained in this form

- I have agreed, or agree to allow the person I am responsible for, to participate in this research study

- I understand that my family doctor may be informed of my participation in this research study

I give permission to Dr. Narod and the study team to contact me by telephone if additional information is needed.

$$
\square_{\text {Yes }} \square_{\text {No }}
$$

I give permission to Dr. Narod and the study team to contact me by telephone/email/mail about future studies.

Yes $\square$ No 


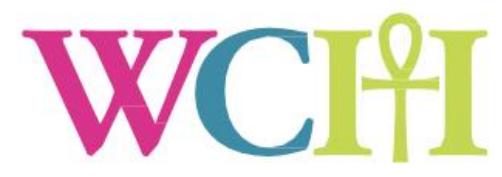

Dr. Steven Narod, MD, FRCPC

Canada Research Chair in Breast Cancer

WOMEN'S COLLEGE HOSPITAL 76 Grenville Street, 6th Floor, Toronto, Ontario M5S1B2

Health care for women $\mid$ REVOLUTIONIZED

By signing this form, I confirm that:

- This study and its purpose has been explained to the participant above

- All questions asked by the participant have been answered

- I will give a copy of this signed and dated document to the participant

Name of person (investigator) obtaining consent

Signature

Date

\section{ASSISTANCE DECLARATION}

The Assistance Declaration provides a mechanism for potential participants who are unable to read the informed consent form (i.e. illiterate, blind or for whom English is their second language) to participate in research studies.

Was the participant assisted during the consent process? Yes

No

The consent form was read to the participant/substitute decision-maker, and the person signing below attests that the study was accurately explained to, and apparently understood by, the participant/substitute decision-maker. The person signing below acted as a translator for the participant/substitute decision-maker during the consent process.

$\mathrm{He} / \mathrm{sh}$ attests that they have accurately translated the information for the participant/substitute decision-maker, and believe that that participant/substitute decision-maker has understood the information translated.

Name of Person Assisting (Print)

Signature

Date

Person obtaining consent

By signing this form, I confirm that:

- This study and its purpose has been explained to the participant named above

-All questions asked by the participant have been answered

- I will give a copy of this signed and dated document to the participant 


\section{Chapter 7. Copyright Acknowledgements}

Permission had been granted for the reproduction of figures under the publisher Ivyspring International Publisher from the following published journal article for use in this thesis:

- Figure 1 from Gorodetska I, Kozeretska I, Dubrovska A. BRCA Genes: The Role in Genome Stability, Cancer Stemness and Therapy Resistance. J Cancer. 2019;10(9):21092127.

Permission had been granted for the reproduction of figures under the publisher Wolters Kluwer Health, Inc from the following published journal article for use in this thesis:

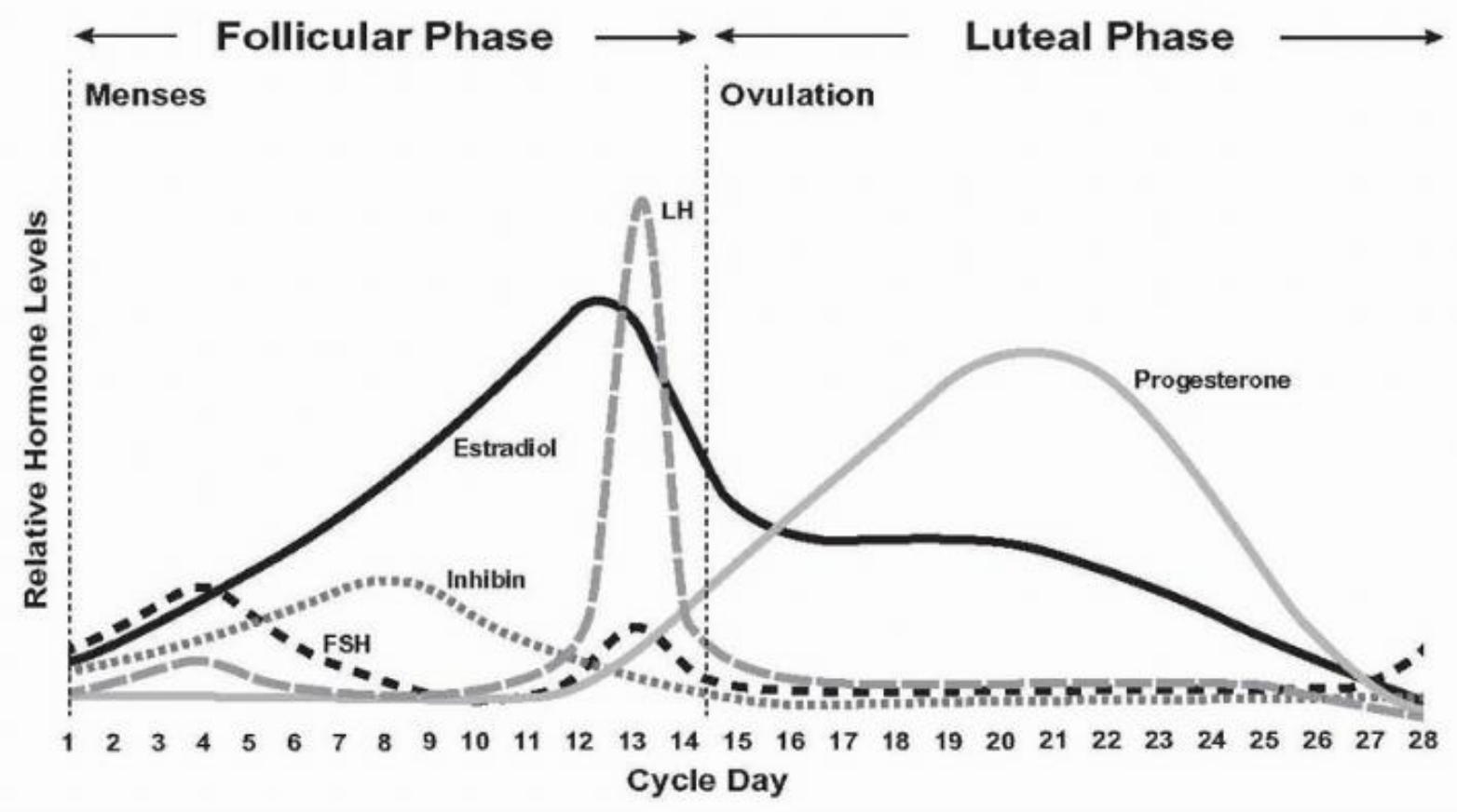

- Figure 2 from Jensen JT, Creinin MD. Speroff \& Darney’s Clinical Guide to Contraception. Sixth edition. Philadelphia, PA : Lippincott Williams \& Wilkins/Wolters Kluwer; 2020.

Permission had been granted for the reproduction of figures under the publisher Elsevier from the following published journal article for use in this thesis:

- Figure 3 from Collaborative Group on Epidemiological Studies of Ovarian Cancer. Ovarian cancer and smoking: individual participant meta-analysis including 28114 women with ovarian cancer from 51 epidemiological studies. The Lancet Oncology. 2012;13(9):946-956.

Permission had been granted for the reproduction of figures under the publisher Springer Nature from the following published journal article for use in this thesis: 
- Table 3 from Schindler AE. Pharmacology of Progestogens. In: Progestogens in Obstetrics and Gynecology. Second edition. Springer International Publishing; 2021. 